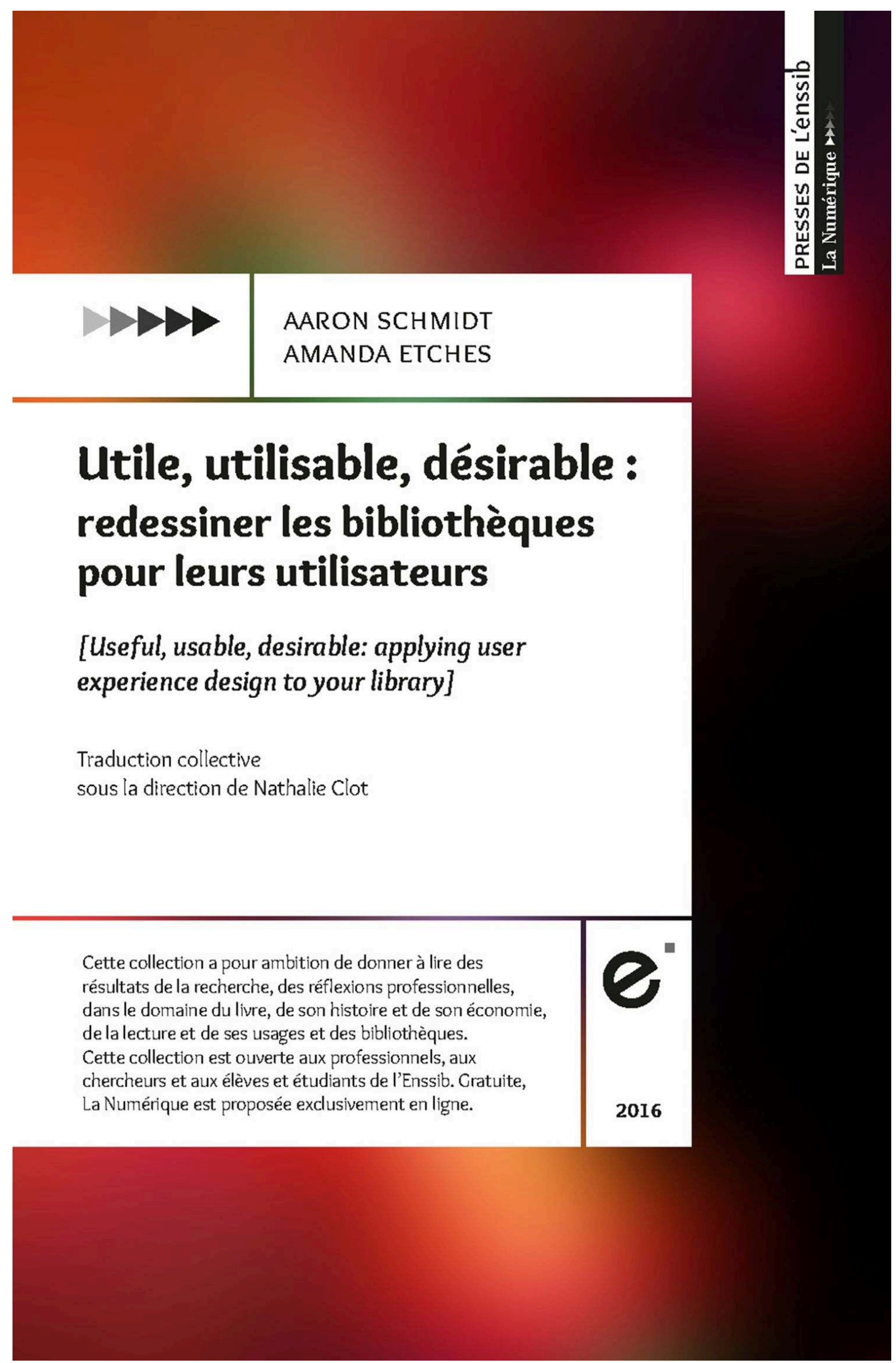




\section{Utile, utilisable, désirable}

Redessiner les bibliothèques pour leurs utilisateurs

\section{Amanda Etches et Aaron Schmidt}

Traducteur : Nathalie Clot

DOI : 10.4000/books.pressesenssib. 1537

Éditeur : Presses de l'enssib

Année d'édition : 2016

Date de mise en ligne : 4 avril 2017

Collection : La Numérique

ISBN électronique : 9782375460535

\section{Sb books}

http://books.openedition.org

\section{Référence électronique}

ETCHES, Amanda ; SCHMIDT, Aaron. Utile, utilisable, désirable : Redessiner les bibliothèques pour leurs utilisateurs. Nouvelle édition [en ligne]. Villeurbanne : Presses de l'enssib, 2016 (généré le 01 février 2021). Disponible sur Internet : <http://books.openedition.org/pressesenssib/1537>. ISBN :

9782375460535. DOI : https://doi.org/10.4000/books.pressesenssib.1537.

(C) Presses de l'enssib, 2016

Conditions d'utilisation :

http://www.openedition.org/6540 


\section{Utile, utilisable, désirable : redessiner les bibliothèques pour leurs utilisateurs}

[Useful, usable, desirable: applying user experience design to your library]

Traduction collective

sous la direction de Nathalie Clot

Cette collection a pour ambition de donner à lire des résultats de la recherche, des réflexions professionnelles, dans le domaine du livre, de son histoire et de son économie, de la lecture et de ses usages et des bibliothèques. Cette collection est ouverte aux professionnels, aux chercheurs et aux élèves et étudiants de l'Enssib. Gratuite, La Numérique est proposée exclusivement en ligne. 


\section{Les auteurs}

Aaron Schmidt a travaillé comme agent d'accueil, été responsable d'une section «jeunes adultes», puis directeur de bibliothèque. Il est actuellement à la tête d'Influx Library User Experience, une entreprise de conseil spécialisée dans l'intégration en bibliothèque $d u$ Design de l'expérience utilisateur. Il est chroniqueur d'une rubrique régulière de Library Journal intitulée «The User Experience » et intervient à la San José State University's School of Library and Information Science. Il est également membre du comité éditorial de Weave: Journal of Library User Experience. Aaron Schmidt tient un blog sur les questions de Design en bibliothèque à l'adresse < http://walkingpaper.org >.

Amanda Etches est responsable du département Discovery \& Access à la University of Guelph Library, au Canada, où elle s'occupe d'accompagner les équipes dans des projets de services orientés vers l'amélioration de l'expérience utilisateur en bibliothèque. Elle travaille également pour Influx. Elle intervient fréquemment sur les questions de design web, d'utilisabilité et toutes les tendances relatives à l'expérience utilisateur. Elle est présente sur twitter à @etches et tient le 
blog < http://e.tches.ca > où vous la verrez un brin coupable d'autant s'amuser à faire ce qu'elle est payée pour faire.

\section{Les traducteurs}

L'équipe de bénévoles français qui a traduit Utile, utilisable, désirable a été coordonnée par Nathalie Clot, directrice de la bibliothèque universitaire d'Angers (BUA), qui a trouvé dans ce manuel une ligne conductrice permettant à la fois d'élaborer un projet de service et de rénover les méthodes de travail. Partager ce texte, d'abord au sein de l'équipe de la BUA, lui a paru être une bonne idée, jusqu'au moment où en en parlant autour d'elle, elle a compris qu'il pouvait intéresser beaucoup d'autres gens. Elle assume l'entière responsabilité des contresens éventuels et lourdeurs résiduelles de la présente adaptation et remercie les Presses de l'enssib du soutien de la première heure accordé à ce projet.

Rien n'aurait été possible sans l'investissement sans faille dans la traduction et/ou les relectures attentives et critiques de:

Nicolas Alarcon, responsable de collection au service commun de documentation de l'université de la Réunion; 
David Aymonin, directeur de l'Agence bibliographique de l'enseignement supérieur (ABES);

Émilie Barthet, directrice adjointe du service commun de documentation de l'université de Lyon 3;

Nicolas Beudon, responsable du département Littérature et philosophie de la Bibliothèque publique d'information (Bpi);

Adeline Desgranges, responsable de la médiathèque Toussaint et des secteurs adultes de la bibliothèque municipale d'Angers;

Frédéric Desgranges, responsable de la bibliothèque universitaire Saint-Serge et des indicateurs à la BUA;

Katrina Kalda, responsable des formations au service commun de documentation de l'université de Tours;

Nadine Kiker, directrice adjointe de la BUA;

Olivier Legendre, directeur adjoint $d u$ service commun de documentation de l'université de Clermont-Ferrand;

Maud Puaud, responsable de la formation des usagers à la BUA;

Maxime Szczepanski, responsable de la bibliothèque universitaire Belle Beille à la BUA. 


\section{Notice}

Utile, utilisable, désirable :

redessiner les bibliothèques pour les utilisateurs

Amanda Etches et Aaron Schmidt

trad. de Nathalie Clot.

Villeurbanne : Presses de l'enssib,

cop. 2016. - 1 vol. (258 p.),

La Numérique; ISSN 2492-9735.

ISBN pdf 978-2-37546-004-7. Gratuit

ISBN epub 978-2-37546-005-4. Gratuit

Dewey : $027.7-025.1$

\section{Rameau :}

Bibliothèques universitaires -- États-Unis

Bibliothèques -- services aux publics

Bibliothèques -- Publics

Bibliothèques -- Gestion

Bibliothèques -- Évaluation

Conception centrée sur l'utilisateur

(9)(1)(ङ) Notice rédigée par la bibliothèque de l'Enssib, 2016.

Copyright (C) 2014

by the American Library Association (ALA)

Titre original : Useful, Usable, Desirable.

Applying User Experience Design to your Library

Auteurs : Amanda Etches et Aaron Schmidt

Éditeur original : the American Library

Association (ALA), Chicago

Traduction copyright (c) 2016 par Presses de l'enssib

Texte intégral 


\section{Sommaire}

Les auteurs et les traducteurs $\quad 2$

Notice 5

Avant-propos: Dessine-moi une bibliothèque 9

1. Introduction à l'UX

(expérience utilisateur) en bibliothèque

1.1 Qu'est-ce que le design de l'expérience utilisateur (User Experience Design)?

1.2 Pourquoi utiliser la notion d'UX en bibliothèque?

1.3 Les trois piliers d'une bonne «expérience utilisateur» 16

1.4 Les huit commandements de l'UX 18

1.5 Comment utiliser ce livre? 25

1.6 Remarques sur la terminologie et l'adaptation en français

2. Analyser les usages: 12 techniques de base

2.1 Analyse des opinions et analyse des comportements 34

2.2 Autres techniques $\quad 40$

2.3 Plus de lectures $\quad 45$

3. La bibliothèque comme lieu 47

3.1 Le bâtiment de la bibliothèque est propre et fonctionne bien 47

3.2 Le bâtiment de la bibliothèque n'est pas encombré inutilement

3.3 Le mobilier répond aux besoins des usagers

3.4 Le bâtiment s'adapte

à des comportements variés

3.5 Les usagers ont facilement accès aux prises de courant 64

3.6 Plus de lectures $\quad 69$

4. Les espaces d'accueil 70

4.1 Les usagers s'approchent

facilement des bureaux d'accueil

4.2 Les besoins changent, les bureaux d'accueil aussi 
4.3 Les gens reçoivent de l'aide

quand et où ils en ont besoin

4.4 Les gens reçoivent le type d'aide

dont ils ont besoin

84

4.5 Plus de lectures

89

5. Règlements et services au public

5.1 Votre bibliothèque a une philosophie des services

5.2 L'équipe connaît et fait vivre

la philosophie des services

5.3 Il y a aussi peu de procédures et de règles que possible

5.4 Les règlements de la bibliothèque donnent des marges d'action

et de décision aux professionnels

5.5 L'équipe de la bibliothèque

est accueillante et serviable

5.6 La qualité du service est homogène au sein de l'institution

5.7 Le service est cohérent

dans toute l'organisation

\section{Signalétique et orientation}

6.1 Votre bibliothèque dispose

d'une charte graphique bien conçue

6.2 Une même charte graphique pour toute la signalétique

6.3 Les différents types

de signalétique sont visuellement distincts

6.4 La signalétique est minimale

6.5 Bannir le scotch

6.6 La signalétique réglementaire est simple, conviviale et courtoise

6.7 La carte de bibliothèque contient des informations utiles et respecte la charte graphique

6.8 Les nouveaux visiteurs repèrent facilement l'organisation générale de la bibliothèque

6.9 Plus de lectures 
7.1 Trouver des documents

et les réserver est facile $\quad 157$

7.2 Facilitez les opérations essentielles $\quad 160$

7.3 La richesse en contenus de votre site est adaptée au temps que vous pouvez y consacrer 168

7.4 Le contenu web est attrayant $\quad 172$

7.5 Rédigez pour le Web 177

7.6 Respectez les conventions

de design du Web 185

7.7 Votre page d'accueil exprime clairement ce que l'on peut faire sur votre site $\quad 189$

7.8 Le site s'adapte à tous les écrans $\quad 193$

7.9 Le site web utilise l'identité visuelle de la bibliothèque

7.10 Vous utilisez les réseaux sociaux

à bon escient $\quad 198$

7.11 Plus de lectures 201

\section{La bibliothèque comme outil de travail $\quad 202$}

8.1 Les équipements et appareils proposés dans la bibliothèque sont adaptés aux besoins, utiles et utilisables 202

8.2 Les collections sont adaptées aux besoins des utilisateurs 208

8.3 Les supports de communication sont utiles 214

8.4 Valoriser ses ressources 218

8.5 Les services de la bibliothèque apportent des solutions aux problèmes des utilisateurs 221

8.6 Plus de lectures 228

9. Conclusion: philosophie, processus et culture 229

9.1 Penser la bibliothèque comme un tout 229

9.2 La démarche de design 233

9.3 La culture de votre organisation 239

9.4 Le mot de la fin 246

Annexes 246

Table des illustrations 250

Index 252

Notes de lecture $\quad 257$ 


\section{Avant-propos: Dessine-moi une bibliothèque}

Les bibliothèques parlent beaucoup et depuis longtemps de mettre l'utilisateur au cœur de la bibliothèque. De la politique documentaire à la formation continue, parler usages, besoins, connaissance des publics sont les lieux communs du moment. Les boîtes à outils, revues professionnelles et comptes rendus d'expériences en ligne foisonnent d'idées d'actions concrètes en la matière.

Alors, pourquoi s'attacher à traduire un petit livre professionnel américain, au titre intrigant? Trois raisons à cela.

\section{Parce que ce livre est utile}

Utile comme peut l'être un livre de recettes au moment de préparer un repas de fête. Utile comme le sont les cours de code et de conduite avant de passer le permis et de se mettre pour la première fois au volant de sa voiture. Utile comme le parent qui encourage son petit en courant derrière le vélo lors de sa conquête d'un équilibre encore précaire.

En effet, sous une forme condensée, il offre une vision globale de bibliothèques créatrices de services et d'une authentique valeur d'usage pour les personnes qui les utilisent. Alors que bien des livres se concentrent sur un seul aspect, qu'il s'agisse de gestion des collections, d'animations, de formations, d'évaluation, de management, d'outils numériques ou d'aménagement des espaces, ce petit livre parle de tout cela à la fois et montre en quoi toutes ces choses sont intimement liées et doivent être pensées ensemble. 
Utile encore, car il propose une méthode de travail, assez facile à mettre en œuvre, qui ouvre des pistes réalistes et rapides d'amélioration.

\section{Parce que ce livre est utilisable}

Il est bref et facile à lire. Il jargonne bien un peu, mais toujours après avoir donné des définitions préliminaires et s'efforce de tout illustrer d'exemples concrets tirés d'expériences de terrain. Vous pouvez n'en lire que quelques chapitres, y picorer, ne regarder que ce qui vous intéresse.

Il est structuré, pratique, progressif. Comme tout vrai bon livre de cuisine, il propose toutes sortes de recettes, classées par ordre de difficulté, que chaque bibliothèque peut réaliser selon ses moyens, le temps dont elle dispose et ses priorités.

Il laisse aussi de la place pour expérimenter et comme bien souvent, les recettes proposées ne sont que des prétextes qui permettront à chaque cuisinier... bibliothécaire d'élaborer l'adaptation locale qui lui convient et de pratiquer son tour de main.

\section{Parce que ce livre est désirable}

Désirable, car comme le disait un des premiers lecteurs français, «partout où on se sait en bonne voie, ça fait chaud au cœur», et qu'il «donne sens à tous les efforts tentés çà et là par les bibliothécaires qui font de l'UX sans le savoir, et qui vont avoir grand plaisir à se reconnaître».

Désirable, car il fait «renaître un rêve, celui d'une relation privilégiée, proche, harmonieuse avec les lecteurs, rêve partagé par beaucoup de professionnels des bibliothèques, et qu'il donne les moyens de lui faire prendre corps».

Désirable enfin, car il peut permettre de partager largement au sein d'un service ou d'un établissement, un certain «désir de 
bibliothèque », une vision, une culture et un vocabulaire commun qui ne soient pas réservés aux quelques happy few lisant couramment l'anglais.

Pour résumer, vous avez entre les mains un ouvrage à la fois terre à terre et idéaliste, pratique et théorique, que vous pourrez aisément partager pour dessiner collectivement une bibliothèque attentive à ses usages et à ses utilisateurs.

Cette alliance de simplicité, de gros bon sens, de méthodes de recherche, de militantisme a séduit les traducteurs qui se sont dit que disposer d'un tel livre en français ne pourrait qu'aider à dépasser des oppositions stériles entre spécialistes de tout poil et serait susceptible de faciliter, sur le terrain, travail commun et choix délibérés.

Traduire et adapter ce texte est né du désir de le partager au sein d'un établissement: il a rapidement paru évident qu'il pourrait être utile et utilisé plus largement, tant en bibliothèques publiques qu'universitaires. Le livre lui-même est loin d'être parfait ou exhaustif: il a hérité de l'original son ton à la fois direct et familier peu fréquent dans la littérature professionnelle francophone, son côté un peu donneur de leçon sur le thème «du passé faisons table rase » et une forme de prosélytisme qui ne laissera pas d'agacer certains lecteurs.

Ces réserves faites, c'était le moment ou jamais de le faire connaître et de le diffuser sans rechercher la perfection: en effet, les méthodes de Design UX, qui sont bien décrites et définies par Amanda Etches et Aaron Schmidt en introduction, sont à la mode dans l'univers marchand. Il n'y avait à ce jour aucun ouvrage en français destiné aux professionnels des bibliothèques désireux d'en savoir plus. L'engagement des Presses de l'enssib et des traducteurs bénévoles a permis de poser ce premier jalon. Chacun pourra ensuite aller plus loin dans une littérature toujours plus abondante. 
Au-delà de l'effet de mode, qui n'aura qu'un temps, ces méthodes peuvent aider, dans la durée, tous ceux, très nombreux, qui cherchent et chercheront à s'adapter au moment présent des bibliothèques, celui des utilisateurs, quelle que soit leur situation de départ.

En effet, l'idée toute simple prônée par Utile, utilisable, désirable est que nous ne pourrons redessiner nos bibliothèques qu'en nous mettant dans la peau de nos utilisateurs. Comment pourrions-nous mieux leur rendre service? Ce n'est pas, en soi, une idée révolutionnaire, ni même neuve. Harper Lee, dans To Kill a Mockingbird écrivait déjà dans les années 1950 en faisant référence à un dicton plus ancien encore: "Atticus was right. One time he said you never really know a man until you stand in his shoes and walk around in them; you never really understand a person until you consider things from his point of view until you climb into his skin and walk around in it. $»^{1}$

Cette vieille idée n’a rien perdu de sa puissance, lorsqu'elle nous permet de réinterroger de manière pragmatique ce que nous pensons savoir, d'adopter une démarche d'essai/ erreur et d'apprentissage mutuel et continu, et de nous adapter en douceur, sans les subir, aux changements de notre environnement.

Ce livre dont vous êtes le héros est écrit à la deuxième personne du pluriel. L'intérêt que vous y trouverez sera ce que vous en ferez ensuite, pour dessiner les bibliothèques d'aujourd'hui et de demain.

Bonne lecture!

Nathalie Clot 


\section{Chapitre 1. Introduction à l'UX (expérience utilisateur) en bibliothèque}

Vous venez d'ouvrir Utile, utilisable, désirable: redessiner les bibliothèques pour leurs utilisateurs.

Ce livre vous permettra de vous faire une première idée de ce qu'est le design de l'expérience utilisateur (UX pour les intimes). S'agit-il d'un cadre conceptuel? Est-ce une méthode qui peut vous permettre d'améliorer et transformer votre bibliothèque?

Être attentif à l'expérience vécue par les utilisateurs, renforcer la place de la bibliothèque dans la cité et en faire un endroit que les gens aiment utiliser est une idée séduisante, mais comment la mettre en pratique, concrètement?

Avant d'entrer dans les détails de ce qu'est le Design UX, ou conception de l'expérience vécue par l'utilisateur en bibliothèque, il est nécessaire de préciser ces notions anglo-saxonnes encore peu répandues dans la culture des bibliothèques françaises. Cette introduction sera aussi l'occasion de parler des partis pris de l'équipe qui a traduit et adapté en français le texte d'Aaron Schmidt et Amanda Etches.

\subsection{Qu'est-ce que le design de l'expérience utilisateur (User Experience Design)?}

La formule est une traduction littérale de l'expression «User Experience Design», souvent abrégée en Design UX en anglais. On pourrait aussi parler de conception orientée vers l'utilisateur, 
mais design a un sens plus large, et inclut une notion de projet, de méthode et de vision globale.

Définir l'«expérience utilisateur», c'est d'emblée se placer sur le terrain du sensible et de la perception: l'UX, c'est ce que quelqu'un ressent et perçoit lorsqu'il utilise un produit ou un service, virtuel ou réel.

Lorsque des individus utilisent un service ou un produit, ils en expérimentent différentes facettes et de nombreux facteurs ont une influence, positive ou négative, sur leur expérience globale.

Un exemple de ces interactions dans la vie quotidienne: vous souhaitez acheter une chemise dans une boutique de prêt-à-porter. Voici une liste - non exhaustive - de ce qui peut avoir un impact sur votre expérience d'utilisateur:

$\odot$ mailing de l'entreprise annonçant une nouvelle collection;

$\odot$ site web de l'entreprise;

$\odot$ vitrine de la boutique;

$\odot$ signalétique et aménagement intérieur;

$\odot$ musique d'ambiance;

$\odot$ amabilité de la vendeuse ou du vendeur et qualité du service client;

$\odot$ disponibilité du produit;

$\odot$ confort et éclairage de la cabine d'essayage;

$\odot$ qualité de la chemise elle-même.

Toutes ces interactions, ces interfaces, ces «points de contact» (touchpoints en anglais), vont avoir un impact sur votre expérience globale au moment de l'achat de cette chemise, puis lorsque vous la porterez. Si un vendeur de chemises comme Arrow ou Eden Park souhaite favoriser une expérience agréable, il a intérêt à prêter attention à tous ces "points de contact». 
Tel est le but du Design UX: les gérer de manière à ce que, pris tous ensemble, ils procurent à l'utilisateur une expérience globale agréable de bout en bout.

\subsection{Pourquoi utiliser la notion d'UX en bibliothèque?}

Une bibliothèque n'est pas comparable à un magasin de prêtà-porter: cela dit, comme dans n'importe quel commerce, elle interagit avec ses utilisateurs via de multiples points de contact. La somme de ces derniers forme l'expérience globale de l'utilisateur qui peut être bonne, mauvaise ou neutre.

Les points de contact en bibliothèque sont légion. En voici une liste déjà longue:

$\odot$ site web;

$\odot$ catalogue;

$\odot$ bases de données;

$\odot$ email;

$\odot$ chat;

$\odot \mathrm{SMS}$

$\odot$ renseignement en ligne;

$\odot$ téléphone;

$\odot$ parking, accessibilité depuis les transports en commun;

๑ bâtiment, propreté générale;

$\odot$ personnel de la bibliothèque;

$\odot$ signalétique;

$\odot$ mobilier / rayonnages; 
$\odot$ collections;

$\odot$ programmation, animations, formations;

$\odot$ ordinateurs, wi-fi;

$\odot$ brochures, affiches, publicités;

$\odot$ carte de bibliothèque;

$\odot$ lettre d’information imprimée ou en ligne.

L'idée que l'expérience des gens puisse mal tourner à tant d'endroits peut vous inquiéter. C'est légitime.

Lorsqu'on cherche à faire vivre une excellente expérience à chaque utilisateur de la bibliothèque, ce qui est complexe, c'est que tout entre en compte. Si les services rendus par votre bibliothèque sont inadaptés aux besoins et que votre bâtiment est sale, le meilleur des services d'accueil ne suffira pas à donner une bonne impression d'ensemble. Si votre bâtiment est magnifique, mais que le personnel est impoli, les utilisateurs de la bibliothèque auront forcément une expérience négative. Le meilleur moyen de s'assurer que chacun ait la meilleure expérience possible de la bibliothèque est d'optimiser l'ensemble des points de contact avec chaque utilisateur.

La route est longue et pavée de difficultés, mais ne paniquez pas et continuez à lire cette introduction.

\subsection{Les trois piliers d'une bonne "expérience utilisateur»}

Être utile. Utilisable. Désirable.

Voilà les trois éléments essentiels requis pour que chaque utilisateur ait une expérience agréable dans votre bibliothèque. Voyez plutôt: 


\section{Utile}

C'est le cœur de la question. Un produit ou un service doit résoudre un problème ou satisfaire un besoin. Si vous achetez quelque chose qui est désirable, mais qui n'est pas utile, un gadget tapeà-l'œil par exemple, vous pourrez l'utiliser quelques jours ou de manière très ponctuelle: il finira probablement par prendre la poussière au fond d'un tiroir. C'est un des concepts clés pour les bibliothèques. Si une bibliothèque n'est pas utile, elle ne peut pas avoir d'importance pour la communauté qu'elle doit desservir.

\section{Utilisable}

À quoi bon être utile si le service est impossible à utiliser ou cause de frustration permanente? On ne pourra en tirer rien de bon. Pensez aux bases de données des bibliothèques. Elles contiennent des informations de qualité, utiles, mais elles sont souvent difficiles à exploiter. Se contenter d'être utilisable ne suffit pas à ce que les gens utilisent réellement un service, mais y contribue à coup sûr.

Des horaires d'ouverture inadaptés peuvent également rendre la plus belle des bibliothèques inutilisable, faute que les utilisateurs puissent s'y rendre quand ils sont disponibles.

\section{Désirable}

Afin de créer réellement un lien avec un produit ou un service, les gens doivent soit en avoir besoin soit vouloir l'utiliser (on a bon espoir que les deux conditions soient réunies). Si votre bibliothèque fournit un service qui est à la fois utile et utilisable, mais que personne ne le sait, ni n'en a envie, vous pouvez aussi bien arrêter de le rendre.

La chose à retenir lorsque vous pensez aux trois piliers d'une bonne UX est que vous ne pouvez pas vous concentrer sur un 
seul des trois éléments. Vous ne pouvez pas non plus décider que, si votre bibliothèque remplit seulement deux objectifs sur trois, vous êtes au-dessus de la moyenne et que c'est largement suffisant.

Utilité, utilisabilité et désirabilité sont liées. Tout ce que vous faites dans votre bibliothèque, chaque service, chaque ressource, chaque interface, chaque espace, doit remplir ces conditions et à la fois servir à quelque chose, être aisé d'accès et donner envie de l'utiliser: faute de quoi, vous ne pourrez pas optimiser l'expérience globale que vous pourriez procurer aux membres de votre communauté.

Ce livre peut vous aider à vous organiser et à travailler en équipe pour y arriver, pas à pas.

Avant d'aller plus loin et d'entrer dans les considérations pratiques, voici encore quelques définitions pour vous permettre de bien appréhender la démarche.

Huit grands principes sous-tendent la vision générale du Design UX. Indissociables, ils en constituent la philosophie sous-jacente et la colonne vertébrale.

\subsection{Les huit commandements de l'UX}

\section{№ 1 : Vous n'êtes pas votre usager}

L'idée selon laquelle le concepteur n'est pas l'utilisateur vient du monde de la recherche sur les interactions entre humain et ordinateur. Transposé aux bibliothèques, cela veut simplement dire que vous ne devez pas concevoir vos espaces, services ou interfaces en vous considérant comme comparable à votre public cible et en pensant «je suis lecteur et rien de ce qui concerne un autre lecteur ne m'est étranger». 
$\mathrm{Au}$ lieu de cela, vous devez concevoir tous vos services en gardant à l'esprit que vous ne ressemblez pas à vos usagers. Contrairement à eux, vous connaissez la bibliothèque de l'intérieur. Vous savez comment les choses sont censées fonctionner. Vous avez une culture professionnelle, des a priori, des connaissances, et une expertise pour tout ce qui se passe en bibliothèque que les autres personnes ne partagent pas. Donc, si vous concevez les choses pour vous-mêmes en tant que bibliothécaires, non seulement les utilisateurs de la bibliothèque partent avec un handicap, mais ils risquent aussi de se retrouver en situation d'échec.

\section{№ 2: L’usager n'est pas défectueux}

Karen Schneider a fait émerger cette notion dans la culture professionnelle des bibliothécaires en 2006, en publiant un manifeste intitulé «L'usager n'est pas défectueux», «The user is not broken» dans le texte (<http://freerangelibrarian.com/2006/06/03/ the-user-is-not-broken-a-meme-masquerading-as-a-manifesto $>$ ).

Revenons quelques décennies en arrière: à l'époque, les gens devaient apprendre à se servir des bibliothèques et avaient besoin d'aide pour le faire. Entre les fichiers papier et la recherche sur serveurs en ligne de commandes, le pouvoir de trouver de l'information était baigné de mystère et réservé à quelques élus triés sur le volet. Seuls les bibliothécaires avaient la clé pour ouvrir l'accès à ce monde défendu. Si un usager s'asseyait inconsidérément devant un terminal de CD-Rom et essayait de trouver comment faire une recherche tout seul, un bibliothécaire intervenait. Le pauvre utilisateur lambda, lui, n'était pas formé à chercher dans les bases de données comme un bibliothécaire et n'avait donc aucune chance d'y parvenir.

De nos jours, les interfaces ne sont plus conçues pour être utilisées par l'intermédiaire des bibliothécaires. Il n'est cependant pas rare d'entendre encore des bibliothécaires trouver des excuses à des 
systèmes de recherche mal conçus en disant «nous aurons juste à montrer aux utilisateurs comment ça marche, et tout ira bien ».

Le design orienté vers l'utilisateur retourne complètement cette notion et dit que, s'il faut enseigner à quelqu'un comment utiliser un outil, alors c'est cet outil qui est défectueux, et non la personne qui l'utilise.

\section{№ 3 : Faites de la recherche sur les usages}

Il est très facile de prendre un morceau de papier et un crayon pour concevoir quelque chose. Le spécialiste en cognition préféré des experts en utilisabilité, Don Norman, cite l'exemple des distributeurs automatiques bancaires dans beaucoup de ses travaux (par exemple, Emotional Design: Why We Love (Or Hate) Everyday Things, non traduit en français).

Quand les premiers distributeurs bancaires ont été conçus, on s'imaginait qu'on avait simplement besoin d'une machine pleine de monnaie et d'une personne munie d'une carte bancaire pour automatiser les retraits d'argent. Le porteur de carte insérerait sa carte dans la machine, taperait son code secret, dirait à la machine combien d'argent il voulait, prendrait les billets dans la fente prévue à cet effet, reprendrait sa carte et repartirait. Facile! Et plein de bon sens. Sauf que, lorsque les premiers distributeurs ont été mis en service, il est arrivé à certaines personnes de prendre l'argent et de partir en oubliant de reprendre leur carte. Si un ingénieur spécialiste des interactions ou un designer ne s'était pas arrêté pour regarder comment les gens utilisaient les guichets automatiques bancaires, les machines que nous connaissons, qui limitent le nombre de cartes oubliées en partant, n'existeraient pas.

En faisant ces recherches fondées sur des observations concrètes, et en apprenant à en savoir plus sur la vie, les préférences, 
les espoirs et les rêves des usagers de votre bibliothèque et la manière dont ils l'utilisent, vous pourrez adapter vos services existants et en créer de meilleurs.

\section{№ 4: Construisez de l'empathie}

Votre vie serait plus facile si vous ne pensiez qu'à vous. Celle des autres le serait beaucoup moins. L'empathie est cette petite chose qui permet de comprendre ce que ressent quelqu'un d'autre et de le partager. En langage courant, l'empathie consiste à se mettre dans la peau de l'autre, ou pour reprendre l'expression imagée anglaise, se glisser dans les chaussures de quelqu'un d'autre et marcher pendant un kilomètre avec. Pour créer des services qui ont du sens et qui donnent envie d'être utilisés, il faut laisser de côté votre point de vue de bibliothécaire et adopter celui des utilisateurs.

Plus encore, il faut accorder de la valeur à ce changement de point de vue. C'est seulement lorsque vous en serez capable que vous pourrez créer d'excellentes expériences pour les utilisateurs.

L'un des meilleurs exemples sur la manière de construire de l'empathie avec les utilisateurs est la journée «Dans la peau d'un usager», une idée lancée en 2008 par Brian Herzog (<http:// www.swissarmylibrarian.net/2008/10/07/work-like-a-patronday >). Le but est d'encourager le personnel de la bibliothèque à expérimenter concrètement les conditions d'utilisation réelles en passant sa journée de travail dans les espaces publics. Cela va du travail sur les ordinateurs en libre accès à l'utilisation des toilettes publiques. Des activités de ce type peuvent aider à penser comme des utilisateurs, premier pas dans la construction d'une empathie réelle envers eux. Le second pas - se préoccuper de leur ressenti - ne peut pas être enseigné et impose d'être vigilant lorsqu'on fait un recrutement en bibliothèque. 


\section{№ 5 : Faites une bibliothèque facile à utiliser plutôt que tape-à-l'œil}

Dans le monde du développement web, dire que quelque chose doit être facile à utiliser plutôt qu'attirer l'attention signifie qu'il faut d'abord s'occuper des fonctionnalités avant de mettre du son et des animations. Ainsi, lorsque vous concevez un site web, vous devez avant tout vous préoccuper de choses comme la vitesse de réaction du site, son temps de chargement et sa navigation, avant d'inclure des éléments comme des galeries d'images, des visuels sur la page d'accueil et des contenus conçus par les usagers. Ces idées de simple bon sens peuvent être facilement extrapolées à tout ce qui peut être un objet de design, des espaces aux services en passant par les bureaux d'information.

\section{№ 6 : Tendez vers l'universel}

Le design universel (UD) a pour objectif de créer des environnements utilisables par tous, jeunes et moins jeunes, bien portants et malades, porteurs de handicap ou non. Le Center for Universal Design de la North Carolina State University a identifié les sept principes de design universel suivants (<www.ncsu.edu/ncsu/ design/cud/about_ud/udprinciplestext.htm >). Le design universel doit donc être:

$\odot$ utilisable par tous;

$\odot$ suffisamment flexible pour s'adapter à une palette variée de préférences et de compétences;

$\odot$ intuitif à utiliser et facile à comprendre, quelle que soit l'expérience ou l'habileté préalable;

$\odot$ visible, afin de communiquer de l'information de manière efficace et implicite à l'usager; 
$\odot$ compatible avec les utilisations erronées, pour minimiser les conséquences négatives s'il est utilisé de manière incorrecte;

$\odot$ peu exigeant en termes d'effort physique;

$\odot$ adapté à la taille de l'utilisateur, à l'espace requis et facile d'accès.

Ces principes sont utiles pour concevoir des environnements tant physiques que virtuels: ils s'adaptent sans difficulté à l'accessibilité en ligne.

\section{№ 7: Faites des choix conscients et délibérés}

Faites un tour dans votre bibliothèque et posez-vous la question suivante: «pourquoi les choses sont-elles organisées ainsi?»

Pourquoi le bureau d'information est-il placé à cet endroit? Pourquoi la collection de référence, si vous en avez encore une, est-elle là? Pourquoi les poubelles et bacs de recyclage sont-ils dans ce coin-ci?

La réponse est bien souvent «parce que ça s'est trouvé mis comme ça, au fil du temps».

Ici intervient une notion radicale: tout dans votre bibliothèque (physique comme virtuelle) doit être conçu en fonction du comportement de l'utilisateur et répondre à une volonté délibérée. Qu'est-ce que cela signifie? Rien ne doit être laissé au hasard, ajouté, ôté ou déplacé sans intention préméditée: chaque aspect physique ou virtuel de vos bibliothèques doit être comme il l'est pour une bonne raison que vous êtes en mesure d'expliquer et de justifier à un instant donné.

Réussir cela est un défi. Vos usagers vous remercieront de l'avoir relevé. 


\section{№ 8 : Pensez de manière globale}

Aaron Schmidt et Amanda Etches racontent avoir fait un jour un atelier sur le Design UX avec un groupe de professionnels des bibliothèques. En fin de séance, un participant a levé la main et demandé: «En quoi est-ce différent de ce que nous avons toujours fait dans les bibliothèques? Nous nous sommes toujours préoccupés de nos usagers. Nous avons toujours été centrés sur nos usagers. Nous rendons un vrai service au public! » Posez la question à n'importe quel personnel de bibliothèque, tout particulièrement à ceux qui travaillent en contact direct avec les usagers. Il y a fort à parier qu'ils vous diront tous la même chose: «Nous rendons un vrai service au public!» Une tendance persistante à confondre le Design UX et le service public parce que ce dernier est plus familier.

Pourtant, le vrai Design UX va beaucoup plus loin qu'un bon service au public. Il tient compte de:

$\odot$ tous les points de contact mentionnés plus haut;

$\odot$ la manière dont les usagers sont traités quand ils entrent dans le bâtiment;

$\odot$ la façon dont ils se sentent dans vos locaux;

$\odot$ la facilité avec laquelle ils peuvent effectuer ce qu'ils sont venus y faire.

Réussir à assurer une bonne qualité globale et à rester également attentif à tous ces points est la clé de voûte d'une approche globale de l'UX.

Suis-je un designer? Ne vous en faites pas. Vous n'avez pas besoin de porter un col roulé et des lunettes carrées pour être un designer. Que vous le vouliez ou non, chaque fois que vous créez 
un règlement, un marque-page, un service dans votre bibliothèque, vous prenez une décision concernant le design. Pensez au design comme à une manière d'organiser les choses pour remplir une fonction particulière. De nombreuses décisions de design en bibliothèques relèvent du design fortuit ou du design par défaut. Ce livre vous permettra de passer à des décisions de design délibérées. Le résultat: une meilleure bibliothèque et des usagers plus heureux. Pour plus d'informations sur le processus de design, voir chapitre 9.

\section{Qu'en pensez-vous?}

Êtes-vous d'accord avec ce qui précède? Non? Vous n'aurez pas envie d'en lire beaucoup plus: la suite du texte contient plein d’idées fondées sur ces principes, ainsi que des propositions concrètes pour traduire en actions cette philosophie.

Si vous êtes d'accord, parfait! Reprenez votre lecture...

\subsection{Comment utiliser ce livre?}

Les chapitres 3 à 8 traitent des différents points de contact en bibliothèque: lieux, services, règles, signalétique, services en ligne, collections et animations. Chaque chapitre vous permettra de partir d'un état des lieux de ce qui se passe dans votre établissement sans vous perdre en considérations théoriques. Cet état des lieux vous aidera à:

$\odot$ comprendre l'importance du sujet et son impact sur l'expérience vécue par les utilisateurs dans votre bibliothèque;

$\odot$ estimer où se situe votre bibliothèque en termes de qualité d'expérience utilisateur;

$\odot$ trouver des idées pour l'améliorer. 


\section{Facile ou difficile? Apprenez à lire dans les étoiles}

Comme dans tout bon livre de recettes, avant chaque suggestion d'amélioration, vous trouverez une indication de son niveau de difficulté, allant de 1 étoile à 3 étoiles. Ces étoiles UX vous permettront de jauger si vous avez le temps et les moyens d'entreprendre une action corrective donnée ou s'il est préférable de vous concentrer sur des objectifs plus faciles à atteindre.

Comme l'indique le tableau ci-dessous, auquel vous pourrez vous référer tout au long de votre lecture, une action 1 étoile est relativement facile et rapide à mettre en œuvre alors qu'une démarche 3 étoiles mettra du temps à aboutir et exigera des moyens importants, notamment en termes d'investissement humain. Si vous débutez complètement, commencer par des actions 1 étoile vous permettra d'engranger rapidement des petits succès encourageants qui pourront donner envie à vos collègues de venir voir de plus près ce que vous faites et de se joindre à votre démarche.

\begin{tabular}{|c|c|c|c|c|}
\hline Niveau & Aperçu & Difficulté & Durée & $\begin{array}{l}\text { Compétences } \\
\text { requises }\end{array}$ \\
\hline 1 étoile & $\begin{array}{l}\text { Assez rapide } \\
\text { et facile à } \\
\text { mettre en } \\
\text { œuvre, en } \\
\text { faisant un } \\
\text { petit effort. }\end{array}$ & $\begin{array}{l}\text { Mineure. } \\
\text { Peut être } \\
\text { mené à bien } \\
\text { à l'initia- } \\
\text { tive d'un seul } \\
\text { membre du } \\
\text { personnel, } \\
\text { juste avec } \\
\text { l'accord } \\
\text { du respon- } \\
\text { sable de ser- } \\
\text { vice. Impact } \\
\text { budgétaire } \\
\text { minime. }\end{array}$ & $\begin{array}{l}\text { Quelques } \\
\text { heures à une } \\
\text { semaine. }\end{array}$ & $\begin{array}{l}\text { Pas de } \\
\text { compétences } \\
\text { particulières. }\end{array}$ \\
\hline
\end{tabular}




\begin{tabular}{|c|c|c|c|c|}
\hline 2 étoiles & $\begin{array}{l}\text { Implications } \\
\text { nombreuses. } \\
\text { Demande } \\
\text { plus de } \\
\text { temps et de } \\
\text { travail pour } \\
\text { réussir. }\end{array}$ & $\begin{array}{l}\text { Modérée. } \\
\text { Peut deman- } \\
\text { der un sou- } \\
\text { tien financier } \\
\text { et organisa- } \\
\text { tionnel pour } \\
\text { réussir. }\end{array}$ & $\begin{array}{l}\text { Une à quatre } \\
\text { semaines. }\end{array}$ & $\begin{array}{l}\text { Certaines } \\
\text { compé- } \\
\text { tences parti- } \\
\text { culières (ou } \\
\text { formations) } \\
\text { peuvent être } \\
\text { nécessaires } \\
\text { pour réussir. }\end{array}$ \\
\hline 3 étoiles & $\begin{array}{l}\text { Exige des } \\
\text { changements } \\
\text { d'organisa- } \\
\text { tion et ne } \\
\text { peut être } \\
\text { accompli que } \\
\text { sur le long } \\
\text { terme. }\end{array}$ & $\begin{array}{l}\text { Majeure. } \\
\text { Exigera un } \\
\text { investisse- } \\
\text { ment signi- } \\
\text { ficatif à } \\
\text { l'échelle de } \\
\text { l'organisa- } \\
\text { tion, un sou- } \\
\text { tien financier } \\
\text { ou un chan- } \\
\text { gement dans } \\
\text { la culture } \\
\text { d'établisse- } \\
\text { ment, voire } \\
\text { les deux. }\end{array}$ & $\begin{array}{l}\text { De quelques } \\
\text { mois à plus } \\
\text { d'un an. }\end{array}$ & $\begin{array}{l}\text { Pourra exi- } \\
\text { ger un large } \\
\text { développe- } \\
\text { ment des } \\
\text { compé- } \\
\text { tences / des } \\
\text { forma- } \\
\text { tions pour } \\
\text { l'ensemble } \\
\text { des } \\
\text { personnels. }\end{array}$ \\
\hline
\end{tabular}

Les étoiles sont une indication générale, qui peuvent s'appliquer à la plupart des bibliothèques, mais aussi varier selon la disponibilité des équipes, l'adhésion de la gouvernance ou la culture actuelle de votre organisation.

\section{Connais-toi toi-même: le CUTI, pour faire le diagnostic de la situation dans votre bibliothèque}

Ce livre part du principe que pour agir, vous devez commencer par bien comprendre votre situation locale. Chaque chapitre vous propose donc des méthodes pour faire l'autodiagnostic de votre bibliothèque sur chaque point de contact. Cela prend la forme d'exercices d'observation, dont les résultats sont «notés » : établir votre note, ou «cote d'utilisabilité », comme nous avons choisi de 
l'appeler, exigera de votre part honnêteté intellectuelle et lucidité, sans prétendre à une objectivité scientifique.

Estimer votre cote d'utilisabilité (CUTI) doit vous aider à mesurer la qualité de l'expérience des utilisateurs de votre bibliothèque. L'objet du CUTI n'est pas de faire du benchmarking et de vous comparer aux autres, mais d'identifier ce que vous faites déjà bien, et ce que vous pouvez encore améliorer.

L'annexe 1 récapitule tous les points de diagnostic. Cette liste a deux objectifs: vous donner une vue globale des enjeux de chaque thème et vous permettre d'y reporter vos résultats.

Vous pourrez utiliser la représentation graphique sous forme de radar pour identifier de manière très visuelle les points forts de l'expérience utilisateur dans votre bibliothèque et les pistes d'amélioration, et en partant de là, vous fixer des priorités et déterminer votre plan d'action personnalisé. Selon une grille de lecture classique, commencer par améliorer des points faibles très faciles à régler paraît plus pertinent que travailler d'emblée sur vos points forts ou sur des points faibles dont l'amélioration promet d'être longue et coûteuse.

Tenir des comptes ne vous intéresse pas? Pas de problème. Si vous n'avez pas pour but de lancer immédiatement une série de projets d'amélioration, vous pouvez vous contenter de lire ce livre pour enrichir votre culture professionnelle.

\section{Quand et qui?}

Il n'y a aucune raison de lire ce livre dans l'ordre des chapitres. Si vous lancez un projet d'amélioration de votre site web, allez tout droit au chapitre 7. Cela dit, ne laissez de côté aucun chapitre! Plus vous optimiserez de points de contact, meilleure sera l'expérience globale des utilisateurs de votre bibliothèque. 
Que vous soyez un loup solitaire de l'UX ou que vous fassiez partie d'un projet d'organisation global, vous pouvez utiliser ce livre. Si vous commencez à peine à améliorer l'UX et si votre culture organisationnelle est coopérative, il est recommandé de former une équipe transversale, rassemblant des représentants de tous les services. Commencez par de petits projets qui pourraient avoir un impact significatif et construisez votre démarche sur ces premiers succès.

Bien que ce livre plaide pour une amélioration d'ensemble de l'expérience utilisateur, vue comme un tout, s'il n'y a aucun espoir, dans votre établissement, de former une équipe UX transversale, vous pourrez quand même trouver des parties de ce livre pertinentes pour votre propre service. Peut-être votre exemple donnera-t-il envie aux autres de s'y mettre!

\subsection{Remarques sur la terminologie et l'adaptation en français}

\section{Le public de la bibliothèque}

Les détails sont souvent importants et la manière dont on parle des personnes qui utilisent la bibliothèque et ses services n'a rien d'anodin. Il y a en effet beaucoup à dire, en anglais comme en français, sur la manière de désigner le public des bibliothèques.

La plupart des bibliothèques publiques utilisent en anglais le terme patron et la plupart des bibliothèques universitaires étudiant. Aaron Schmidt et Amanda Etches discutent longuement du mot patron, qu'ils n'aiment pas et dont le sens en français rendait de toute manière l'usage impossible pour cette adaptation.

Lecteur, encore très utilisé dans les bibliothèques françaises, réduit l'utilisateur de la bibliothèque à la seule partie de la communauté qui «lit des livres». De facto, on peut être utilisateur 
d'une bibliothèque sans pour autant être lecteur. Ne parler que de lecteurs revient à exclure sémantiquement une partie du public potentiel.

Usager est le terme le plus fréquent, et c'est un bon terme lorsqu'on parle de manière générale de l'UX et du service public, mais quand il s'agit de personnes réelles, le mot est un peu impersonnel. Et puis, c'est plein de sous-entendus. Les usagers peuvent être vus comme des individus qui ne donnent rien en retour. Un usager attend, est passif. Usager s'utilise en interne, entre collègues, mais on ne s'adresse jamais à quelqu'un dans la bibliothèque en utilisant le mot usager. Pourquoi? Parce que personne n’a envie d'être appelé usager.

Le mot utilisateur/utilisatrice présente l'avantage de contenir la notion d'utilité. L'utilisateur/utilisatrice, c'est celui ou celle qui utilise la bibliothèque, et à qui la bibliothèque est utile. C'est donc le terme privilégié dans cette adaptation en français pour évoquer l'UX, l'expérience utilisateur, et parler de ceux et celles qui utilisent les services des bibliothèques.

Parler des utilisateurs de la bibliothèque comme étant ses clients était exclu par les auteurs américains. Même s'il est parfois incontournable, comme dans l'expression service client, ce mot ne convient guère, car il met l'accent sur une relation commerciale. Client est un terme de l'économie marchande qui ne reflète pas la dimension de service public des bibliothèques. Les bibliothèques doivent traiter ceux qui les fréquentent sans attendre de paiement en retour et également les désigner sans laisser planer cette équivoque.

Le terme proposé par les auteurs est member tout court. C'est le point le plus délicat de cette adaptation, car une première tentative de traduction littérale émaillée de membres n'a pas franchi la barrière de la première relecture. 
Il a pourtant paru très important, en adaptant ce texte, de garder la notion que l'institution est au service d'un ensemble de personnes qui va bien au-delà de ses seuls utilisateurs. En France, le terme souvent utilisé, notamment en politique, est celui de cité, au sens de communauté des citoyens. L'usage de citoyens aurait donné une tonalité un peu «Révolution française » à l'ensemble du texte.

L'expression «membre de la communauté à desservir» est pertinente, à condition de ne pas prêter le flanc à une imagerie communautariste où servir une communauté particulière reviendrait à en exclure une autre. Elle déforme un peu le sens donné à member par Amanda Etches et Aaron Schmidt pour qui le mot évoque l'idée d'une appartenance, voire d'une forme d'appropriation. Le terme member implique que quelqu'un a fait un choix délibéré. Vous pouvez devenir membre d'une organisation, et y jouer un rôle. Qu'on soit membre d'un club, d'une association, d'un groupe de musiciens, d'un parti, la notion de participation et d'engagement dans l'institution est capitale. Faute de pouvoir adopter, pour la fluidité de lecture en français, le mot membre dans son acception "bienvenue au club», les traducteurs ont choisi une adaptation multiple et métissée: utilisateurs, public, usagers, gens, personnes, membres de la communauté à desservir, citoyens, cité, étudiants à l'occasion. Tous ces termes permettent d'éviter les répétitions, et traduisent l'idée selon laquelle les bibliothèques ne sont pas au service d'individus désincarnés, les usagers, mais forment des collectifs vivants, composés de personnes diverses en interactions permanentes, aux besoins et désirs riches et multiples.

\section{Partis pris de l'adaptation française}

Le texte a été traduit par une équipe de bibliothécaires, néophytes de la traduction. Les auteurs, que nous remercions de leur confiance, ont donné leur accord pour que leur texte soit adapté, 
autant que de besoin, pour faciliter sa réception au sein de la communauté professionnelle francophone.

La fidélité à la lettre a donc souvent cédé le pas à celle de l'esprit et à la recherche de la plus grande fluidité de lecture possible. $\mathrm{Au}$ fil du texte, traducteurs et relecteurs ont pu ajouter des exemples de leur cru, enlever quelques allusions à des situations typiquement américaines incompréhensibles hors contexte, ou rogner quelques répétitions.

Pour les termes techniques du Design UX, les traducteurs ont choisi de reprendre ceux utilisés par Carine Lallemand et Guillaume Gronier dans leur manuel Méthodes de Design UX'1. Lors de la première occurrence, le terme anglais a été repris en italiques et entre parenthèses.

Certains choix sémantiques sont des créations délibérées: les auteurs utilisaient le terme check-up pour introduire tous les exercices d'audit interne proposés. Le terme "autodiagnostic» nous a paru judicieux pour traduire le sous-entendu médical de check-up, sans lui associer le côté cravaté et normatif de l'auditeur aux comptes.

Chacun des diagnostics est associé à une valeur numérique. Les auteurs parlent indifféremment de note, de score ou d'évaluation. Tous ces termes étant connotés de manière négative dans le contexte français, après maints tâtonnements, et conscients de filer la métaphore médicale, nous avons introduit la notion de cote d'utilisabilité, abrégeable en CUTI. Cette notion n'a rien de scientifique et est juste un outil utile pour mesurer les marges d'amélioration dans les différents secteurs d'activité de la bibliothèque. 
L'objectif de l'équipe qui a traduit le livre était bien entendu de rendre cette traduction la plus utile, utilisable et désirable possible. Puissions-nous y être parvenus, malgré d'inévitables maladresses, répétitions et anglicismes résiduels, voire néologismes un peu barbares lorsqu'aucun mot usuel ne permettait de traduire exactement les idées présentées par Amanda Etches et Aaron Schmidt. 


\section{Chapitre 2. Analyser les usages: 12 techniques de base}

Le pivot pour créer du plaisir et de l'engagement chez les utilisateurs est d'étudier quels sont leurs besoins, leurs préférences, leurs espoirs et leurs rêves. On ne peut y parvenir qu'en mettant en œuvre des enquêtes systématiques et cohérentes sur les usages et les usagers. Vous trouverez dans ce chapitre plusieurs techniques classiques: à l'inverse de la plupart des ouvrages sur l'UX, le Design UX ou l'utilisabilité, celui que vous avez entre les mains n'est pas organisé en fonction de ces techniques. Elles sont systématiquement mises en contexte, là où elles peuvent être les plus utiles, d'après les problèmes qu'elles aident à résoudre.

Les techniques d'analyse des usages méritent tout de même une place à part: tout ce chapitre leur est dédié. Après chaque description, volontairement brève, vous trouvez des suggestions d'emploi faisant référence aux chapitres 3 à 8 .

\subsection{Analyse des opinions et analyse des comportements}

Il existe deux sortes de recherches sur les usages et les usagers: l'analyse des opinions et l'analyse des comportements. Comme son nom l'indique, l'analyse des opinions révèle les préférences, les représentations, ce que disent explicitement les utilisateurs. L'analyse des comportements met en lumière ce que font les personnes et la manière dont elles se conduisent en réalité.

Parfois, vous avez besoin de mesurer la satisfaction de vos utilisateurs sur un point (disons, un nouveau service ou une nouvelle 
ressource) et d'autres fois vous voulez en savoir plus sur la manière dont ils utilisent réellement quelque chose (une nouvelle interface ou un bureau d'information, par exemple). Dans l'absolu, une méthode n'est pas meilleure que l'autre: certaines techniques permettent de connaître les préférences des utilisateurs, d'autres ce qu'ils pensent et d'autres encore ce qu'ils font réellement. Il importe de choisir la technique la plus adaptée aux questions que vous vous posez.

Il faut rester conscient de certains biais, inhérents à chaque technique. En tant que discipline, le Design UX accorde plus de valeur à l'analyse des comportements qu'à celle des opinions. Cela s'explique: le design a pour objectif de concevoir, de manière délibérée, des interfaces, outils, services et espaces adaptés à la manière dont les usagers les utilisent réellement, plutôt qu’à comment ils déclarent les utiliser. Cela ne veut pas dire qu'il faut exclure les méthodes de recherche d'opinion, ni prôner un usage exclusif des méthodes comportementales.

Il faut juste être au clair sur si voulez connaître l'opinion qu'ont les individus sur quelque chose que vous faites (ou souhaitez faire) et si vous avez besoin d'en savoir plus sur la manière dont ils utilisent vos outils pour atteindre leurs buts.

Un bon programme d'analyse des usages emploie les deux types de méthodes pour fournir une compréhension complète de votre public, et permettre de prendre des décisions éclairées, en toute connaissance de cause.

\section{Méthodes d'analyse des opinions}

\section{Sondages (surveys)}

Il n'y a sans doute pas grand-chose à dire sur les sondages que vous ne sachiez déjà. Les bibliothèques connaissent bien les sondages: elles en font depuis des années, partagent des outils 
communs et certains types de bibliothèques disposent d'enquêtes standardisées, comme Libqual en bibliothèque universitaire, utilisées pour collecter des données longitudinales et comparer les résultats entre établissements et au fil du temps. L'accent doit être mis sur un point: un sondage est une méthode d'analyse quantitative des opinions. Cela signifie qu'il est bien adapté pour collecter des données sur les représentations et les opinions, mais ne l'est guère pour analyser les comportements réels d'un usager.

Vous trouverez au chapitre 4, Espaces d'accueil, un diagnostic faisant appel à un sondage (4.3 Les gens reçoivent de l'aide quand et où ils en ont besoin).

\section{Focus group (focus group)}

Un focus group consiste à mettre ensemble quelques personnes utilisatrices de la bibliothèque, et à les faire participer à une discussion guidée sur un sujet spécifique. En tant que méthode de recherche sur les attitudes, les focus groups sont une bonne manière d'engager les usagers dans la discussion, de vous informer de leurs besoins et de leurs objectifs vis-à-vis de la bibliothèque et d'avoir un retour sur leurs opinions. Pour tirer le meilleur profit de votre focus group, assurez-vous d'avoir un petit groupe (pas plus de huit participants+le modérateur), que le thème choisi soit précis (par exemple, posez des questions sur un service spécifique ou une ressource) et ciblez vos questions de manière à révéler des attitudes et des opinions. Un travers répandu des focus groups, auquel il faut être attentif, est la dynamique de groupe qui se met en place lorsqu'une seule personne monopolise la parole. Faites attention aux personnes qui généralisent leur propre point de vue, et veillez à entretenir leurs échanges de points de vue avec les autres.

Vous trouverez au chapitre 8, La bibliothèque comme outil de travail, un exemple d'utilisation de focus group (8.2 Les collections sont adaptées aux besoins des utilisateurs). 
Entretien avec un usager (user interviews)

Comme son nom l'indique, un entretien avec un usager est un simple face-à-face, sous forme de conversation, avec des questions et des points clés à aborder. En termes de méthodologie, les entretiens avec un usager sont à cheval entre l'analyse des opinions et celle des comportements, ce qui en fait une de nos méthodes préférées. Un entretien bien mené peut vous permettre de rassembler quantité d'informations tant sur les opinions d'un usager que sur son comportement. Grâce à son côté très ouvert, vous pouvez, au fil de la conversation, la mener où vous avez besoin d'aller et clarifier au fur et à mesure les points abordés.

Le chapitre 8, La bibliothèque comme outil de travail, fait appel aux entretiens pour l'autodiagnostic (8.5 Les services de la bibliothèque apportent des solutions aux problèmes des utilisateurs).

\section{Méthodes d'analyse des comportements}

\section{Enquête contextuelle (contextual enquiry)}

L'enquête contextuelle consiste à regarder les personnes dans leur environnement et lors de leurs activités habituelles. La meilleure manière de comprendre vos usagers est d'observer comment ils se comportent. Il est tentant de penser qu'il suffit de regarder et d'écouter. Or, on ne mène pas une enquête contextuelle en se promenant, sans préparation, dans la bibliothèque. Regarder quelqu'un utiliser l'automate de prêt sur le chemin de la machine à café ne permet pas de dépasser le stade de l'anecdote.

L'enquête contextuelle consiste à observer, pendant un laps de temps précis, plusieurs utilisateurs lorsqu'ils tentent d'accomplir une tâche spécifique. Elle requiert un mélange d'hypothèses, d'observations, de prise de notes rapide, et nécessite de prendre le temps de synthétiser ces données et de réfléchir aux actions à entreprendre. 
Le chapitre 3, La bibliothèque comme lieu, propose un exemple d'enquête contextuelle pertinente (3.4 Le bâtiment s'adapte à des comportements variés).

\section{Cartes d'itinéraires (journey maps)}

Les cartes d'itinéraires décrivent les stratégies et trajets utilisés par les personnes pour mener à bien une tâche, ou lorsqu'elles utilisent un service en particulier. Ces cartes sont souvent des représentations graphiques des multiples points de contact et des actions qui les connectent les uns aux autres. Par exemple, une bibliothèque peut cartographier le processus d'une demande de réservation. Ou, mieux encore, vous pouvez demander à un ou plusieurs de vos usagers de cartographier ce processus à votre place, ce qui en fait une méthode de recherche comportementale très utile.

Pour en revenir à l'exemple d'une demande de réservation: les points de contact et actions pour mener à bien cette tâche peuvent se résumer ainsi:

$\odot$ s’identifier sur le site de la bibliothèque;

$\odot$ faire une demande de réservation via le site web de la bibliothèque;

$\odot$ recevoir un e-mail de notification;

$\odot$ se rendre à la bibliothèque;

$\odot$ entrer dans le bâtiment;

$\odot$ se rendre jusqu'à l'étagère des réservations ou au guichet;

$\odot$ localiser le document ou attendre son tour;

$\odot$ se rendre jusqu'au guichet de prêts / retours s'il est distinct du premier ou à l'automate de prêt; 
$\odot$ avoir une interaction avec l'agent au guichet ou faire une transaction sur l'automate de prêt;

$\odot$ sortir du bâtiment.

Une fois que vous avez énuméré et cartographié l'ensemble de ces points, il devient beaucoup plus facile d'identifier les points de contact spécifiques qui sont une cause de friction ou de blocage. Une fois que vous connaissez tous les points de friction, vous pouvez cibler ceux que vous devez spécifiquement retravailler. Le résultat final est une amélioration globale de l'expérience vécue par chaque utilisateur, alors qu'on s'est contenté d'améliorer seulement quelques-uns des points sélectionnés.

Un exemple classique d'utilisation des cartes d'itinéraire est présenté au chapitre 6, Signalétique et orientation (6.8 Les nouveaux visiteurs repèrent facilement l'organisation générale de la bibliothèque).

\section{Tests d'utilisabilité}

Les tests d'utilisabilité sont étroitement apparentés à l'enquête contextuelle: la plupart des tests d'utilisabilité impliquent d'observer un usager qui tente d'accomplir une tâche spécifique ou une série de tâches prescrites. Les tests d'utilisabilité diffèrent de l'enquête contextuelle sur un point: il s'agit souvent d'une forme guidée, non spontanée d'interaction. L'organisateur du test interagit activement avec l'usager et lui demande de mener à bien les tâches spécifiques qui doivent être observées et testées. Les tests d'utilisabilité sont très utiles pour mettre à l'épreuve les interfaces, les sites web et tous autres environnements virtuels et souligner ce qui marche et ce qui ne marche pas. Vous pouvez tout aussi bien mener un test d'utilisabilité dans un environnement physique: par exemple, vous pouvez tester l'utilisabilité de votre signalétique en demandant à des utilisateurs de trouver des 
lieux spécifiques dans votre bibliothèque et en observant comment ils s'y prennent.

Bien entendu, une description détaillée de l'organisation des tests d'utilisabilité vous sera proposée au chapitre 7, La présence en ligne (7.2 Facilitez les opérations essentielles).

\section{Sondes culturelles ou enquête ethnographique allégée} (cultural probes)

Les sondes culturelles sont une méthode de collecte de données qui permet de rassembler des informations sur une population test pendant une période donnée. C'est une forme allégée d'enquête ethnographique: l'enquêteur demande aux sujets de lui fournir d'eux-mêmes des données sous la forme de comptes rendus subjectifs de type journal intime, de blog, de photographies, de vidéos sur leurs activités pendant une période donnée. Ces sondes culturelles sont en fait des tranches de vies documentées dans un but spécifique.

Les sondes culturelles sont une bonne manière de collecter les données sur le long terme et de mettre en lumière représentations, attitudes aussi bien que des comportements.

Une illustration pratique de cette technique est décrite au chapitre 5, Règlements et services au public (5.7 Le service est cohérent dans toute l'organisation), tout au long du chapitre 8, La bibliothèque comme outil de travail et du chapitre 9, Conclusion: philosophie, processus et culture.

\subsection{Autres techniques}

Il y a quelques autres techniques dans ce livre qui n'entrent ni dans les catégories d'analyse des opinions ni dans l'analyse des comportements et deux ou trois autres qui ne sont absolument pas des méthodes de recherche. Elles sont néanmoins bien utiles pour 
construire une approche plus attentive à l'utilisateur dans votre manière de concevoir vos interfaces, vos services et vos espaces.

\section{Tri de cartes (card sorts)}

Le tri de cartes est une méthode de conception participative utilisant une série de cartes et d'intitulés pour déterminer les préférences d'un usager sur la manière d'organiser quelque chose. Le plus souvent, les tris de cartes sont utilisés dans les processus de développement web lorsque les développeurs conçoivent la navigation d'un site et prennent des décisions sur les intitulés des menus de navigation. Dans ce cas particulier, vous pouvez organiser un tri de cartes avec quelques utilisateurs, pour vous aider à déterminer comment structurer l'architecture et la navigation de votre site, et comment nommer vos menus de navigation de premier niveau.

Les tris de cartes sont également utiles pour d'autres choses que le développement web: il est possible de les utiliser avec vos usagers à chaque fois que vous faites appel à eux sur la manière d'organiser ou de nommer quelque chose, par exemple lorsque vous mettez en espace une collection ou choisissez les intitulés d'une signalétique directionnelle.

Le chapitre 7, La présence en ligne, vous propose plusieurs cas pratiques d'usage du tri de cartes (7.5 Rédigez pour le Web).

\section{Personas (personas)}

Les personas sont des personnages fictifs incarnant le public d'un établissement. Connaître votre public est la clé pour développer services et ressources utiles et pertinents. Cependant, même avec un programme bien développé de recherche sur les usages dans votre bibliothèque, il est souvent facile d'oublier qui sont vraiment les membres de la communauté que vous desservez et quels sont leurs buts. 
C'est là que les personas entrent en scène. Il s'agit d'un bon moyen d'être attentif aux utilisateurs lorsque vous mettez en place quelque chose. Une fois que vous avez fait suffisamment d'analyses pour savoir qui sont vos utilisateurs, ce qu'ils attendent de la bibliothèque, et quels sont leurs besoins, vous pouvez créer une galerie de personas pour représenter les différents segments de votre public: trois à cinq personas feront l'affaire. Ces personnages fictifs sont des constructions intellectuelles qui intègrent des informations démographiques (nom, âge, sexe, etc.) et des données synthétisant comportements, motivations et besoins de chaque persona. Vous pouvez ainsi avoir une persona décrivant les comportements et préférences de «Jonathan, 28 ans, BAC+10, ATER, ne fréquente pas la bibliothèque, mais est un gros utilisateur de documentation en ligne et prescripteur d'achat pour ses étudiants de TD» ou de «Samantha, 14 ans, qui vient à la bibliothèque lire ses mangas préférés le dimanche après-midi avec des copines». Le chapitre 8, La bibliothèque comme outil de travail (8.3 Les supports de communication sont utiles) propose une mise en pratique des personas.

\section{Test A / B}

Comme son nom l'indique, le test A/B est une manière de tester de petites différences de conception, avec des groupes distincts d'usagers, pour mesurer le succès de chaque formule. Cela peut paraître un peu compliqué, mais, en fait, c'est tout simple.

Imaginez un formulaire sur votre site web, avec deux boutons à la fin, l'un intitulé «Annuler» et l'autre «Soumettre». Si votre équipe web n'est pas sûre de la couleur la plus adaptée à chacun de ces boutons, vous avez une bonne occasion pour mettre en place un test $\mathrm{A} / \mathrm{B}$.

Pour faire le test, vous pourrez concevoir deux versions de la même page (version A et version B), avec deux options de couleurs différentes pour chaque bouton. Vous pourrez alors proposer de 
manière aléatoire l'une ou l'autre version de cette page sur votre site web et mesurer leur efficacité lorsque les individus doivent compléter et soumettre le formulaire. Au bout de quelques jours, vous aurez collecté suffisamment de données sur les deux versions du formulaire et vous aurez probablement une idée plutôt bonne de la version qui fonctionne le mieux. Le test A/B est particulièrement utile lorsque vous développez quelque chose de virtuel comme une page web, mais vous pouvez aussi l'utiliser pour les outils imprimés de promotion de votre bibliothèque ou quand vous testez des échantillons de mobilier avant d'aménager des espaces.

\section{Test de 5 secondes}

Comme son nom l'indique, un test de 5 secondes est un test rapide consistant à montrer aux testeurs une page pendant 5 secondes, puis de leur demander dans la foulée ce dont ils se souviennent. Si vous vous souvenez d'avoir joué enfant à ces jeux où vous deviez, en temps limité, retrouver sur un plateau autant d'objets que possible, vous connaissez bien ce test. L'objectif principal d'un test de 5 secondes: mettre en évidence les éléments les plus marquants d'une page web.

Vous trouverez une suggestion d'application de ce test au chapitre 7, La présence en ligne (7.7 Votre page d'accueil exprime clairement ce que l'on peut faire sur votre site).

\section{Audit de contenu (content audit)}

Un audit de contenu consiste à faire une liste de tous les contenus de votre site web. Vous avez bien compris: vous devez recenser chaque page, chaque PDF, chaque image ou vidéo, chaque formulaire, etc., dans un grand tableau. Seul un audit de contenu vous aidera à prendre la pleine mesure de ce qui se trouve sur votre site web pour l'évaluer. 
Un audit de contenu peut être quantitatif: il s'agit alors de faire la liste de chaque page avec son titre et son URL. Il donne une vision globale des contenus de votre site et est utile pour mettre en place un calendrier de mise à jour.

Vous pouvez aller beaucoup plus loin, et créer un audit qualitatif qui vous permettra d'évaluer chaque contenu présent sur votre liste selon différents critères. Par exemple, est-il utile? utilisable? à jour? écrit pour le Web?

Faire un audit de contenu est un gros travail, surtout si votre site se développe anarchiquement. Mais, si l'on considère que diffuser du contenu est la raison même pour laquelle vous avez un site web, les audits de contenus sont des outils dignes d'intérêt.

La démarche d'audit de contenu sera présentée en détail, avec des exemples concrets, au chapitre 7, La présence en ligne (7.4 Le contenu web est attrayant).

\section{L’enquête sociodémographique}

Il existe enfin un type d'enquête ne relevant ni de l'étude des comportements ni de celle des attitudes: l'enquête sociodémographique. Les enquêtes par questionnaire incluent presque systématiquement des questions de ce type: sexe de la personne interrogée, niveau de diplôme, lieu de résidence, âge... Pour obtenir ces données, les bibliothèques universitaires françaises peuvent s'appuyer sur les enquêtes fouillées de l'Observatoire de la vie étudiante (<http://www.ove-national.education.fr/>); les bibliothèques municipales, sur celles de l'Observatoire de la lecture publique (<http://www.observatoirelecturepublique. fr/observatoire_de_la_lecture_publique_web/ >).

Ces données prennent tout leur sens dès lors qu'elles sont corrélées avec des opinions, des attentes ou des comportements. Connaître la pyramide des âges de vos usagers, le nombre 
d'étudiants en master professionnel et donc susceptibles de partir en stage dès le mois de mars, ou le ratio hommes / femmes sur un campus vous permettra d'accroître la taille des espaces et des collections pour les personnes retraitées, d'adapter vos horaires d'ouverture au calendrier de la vie étudiante ou, très prosaïquement, de prendre conscience qu'il est urgent d'accroître l'offre de toilettes publiques pour les femmes. Ces données sont également précieuses au moment de la création de personas.

\subsection{Plus de lectures}

Curedale R., Design Thinking: Process and Methods $2^{\text {nd }}$ Edition, Design Community College Inc., 2016.

Foster N.F., Gibbons S., Studying Students: The Undergraduate Research Project at the University of Rochester, Chicago, Association of College and Research Libraries, 2007.

Hall E., Just Enough Research, New York, A Book Apart, 2013.

Halvorson K., Rach M., Content Strategy for the Web, Berkeley, CA, New Riders, 2012.

Kissane E., The Elements of Content Strategy, New York, A Book Apart, 2011.

Kuniavsky M., Observing the User Experience: A Practitioner's Guide to User Research, $2^{\mathrm{e}}$ éd., Waltham, MA, Morgan Kaufmann, 2012.

Mulder S., Yaar Z., The User Is Always Right: A Practical Guide to Creating and Using Personas for the Web, Berkeley, CA, New Riders, 2007.

Portigal S., Interviewing Users: How to Uncover Compelling Insights, New York, Rosenfeld Media, 2013.

Portigal S., Interviewing Users: Uncovering Compelling Insights. [En ligne] < http://fr.slideshare.net/steveportigal/interviewingusers-uncovering-compelling-insights-la-ux-meetupixda-la >. 
Rubin J., Chisnell D., Handbook of Usability Testing: How to Plan, Design, and Conduct Effective Tests, Indianapolis, Wiley, 2008. [En ligne] < http://www.books24x7.com/marc.asp?bookid=25203>.

Spencer D., Garrett J.J., Card Sorting Designing Usable Categories, New York, Rosenfeld Media, 2009. [En ligne] < http://proquest. safaribooksonline.com/?fpi=9781457102400 >. 


\section{Chapitre 3. La bibliothèque comme lieu}

Dès l'introduction de ce livre, l'accent a été mis sur l'importance d'adopter une démarche globale. L'UX ne se résume pas, comme on le pense encore souvent, à avoir un site web fonctionnel et facile à utiliser. C'est aussi une histoire de lieux, d'atmosphère, d'odeurs et d'univers sonore. Ce chapitre va parler de tout ça et vous offrir plusieurs pistes à explorer pour proposer des espaces agréables à utiliser.

\subsection{Le bâtiment de la bibliothèque est propre et fonctionne bien}

\section{Pourquoi c'est important}

\section{Difficulté *}

Votre bâtiment doit être propre et fonctionnel. Ce doit être une de vos priorités. Votre bâtiment incarne la bibliothèque dans le monde physique: il a, en tant que tel, de l'importance et influence de manière majeure la perception de l'institution.

Si vous avez l'intention que les utilisateurs de votre bibliothèque se sentent bien, alors que votre bâtiment est abîmé, délabré ou sale, il va falloir proposer des services extraordinaires pour compenser l'impact négatif de cet environnement physique déplaisant. Un bâtiment bien équipé et propre ne saurait garantir, à lui seul, que les usagers y viennent, s'y sentent bien et utilisent les services proposés, mais y aidera, c'est sûr. 


\section{Autodiagnostic}

Voici une liste qui vous permettra de faire un premier état des lieux de votre bâtiment. Vous êtes sans doute capable de dresser tout seul un bilan objectif, mais vous pourrez ainsi pousser l'exercice un peu plus loin. Demandez à quelques usagers de vous aider. Leur regard neuf révélera des choses que vous pourriez ne plus remarquer. Utilisez un système de notation simple:

0 - faible, nécessite des améliorations importantes;

1 - correct, de petites améliorations seraient souhaitables;

2 - exemplaire, pas d'améliorations possibles.

\section{Ameublement}

L'ameublement doit être propre et en bon état. Assurez-vous que les textiles et revêtements ne soient ni tachés ni déchirés et qu'il n’y ait pas de pièces cassées.

\section{Toilettes}

Il s'agit d'un sujet sensible, qui peut paraître trivial, mais qui revêt au quotidien une importance majeure. L'état de vos sanitaires joue un rôle décisif dans la bonne ou la mauvaise opinion que les usagers se font de votre bibliothèque. Les sanitaires doivent être propres et nets, correctement éclairés, bien situés et ne jamais manquer des consommables nécessaires. Le calendrier de nettoyage va dépendre de la taille de votre bibliothèque et du nombre de sanitaires, mais, en tout état de cause, ces derniers doivent être souvent nettoyés. Les personnels de la bibliothèque utilisent-ils des sanitaires spécifiques pour éviter d'utiliser ceux destinés au public? Si oui, c'est mauvais signe.

\section{Zones fumeurs}

Depuis qu'il est interdit de fumer à l'intérieur des bâtiments publics et des bureaux, les espaces situés à proximité des issues accueillent 
tous ceux qui sortent fumer une cigarette. Ces zones fumeurs sontelles convenablement équipées et régulièrement nettoyées? Les cendriers sont-ils assez nombreux, bien identifiés et faciles d'utilisation? Est-il possible de matérialiser une zone fumeur protégée de la pluie qui ne soit pas immédiatement devant les sas d'entrée afin d'éviter que le hall de la bibliothèque ne sente le tabac froid? Être attentif à tous ces points est important pour améliorer l'expérience de vos utilisateurs, fumeurs comme non-fumeurs.

\section{Murs et vitrages}

Observez les murs et vitrages de la bibliothèque comme si vous étiez en train d'inspecter une pièce récemment réaménagée de votre maison. Répondent-ils à vos exigences? Les détails suivants altèrent l'apparence générale de votre bibliothèque:

$\odot$ trous;

$\odot$ morceaux de ruban adhésif restés en place après décollage des affiches;

$\odot$ marques laissées par les meubles;

$\odot$ dégâts des eaux ou autres dégradations;

$\odot$ graffitis;

$\odot$ peinture mal appliquée, écaillée ou noircie;

$\odot$ traces de saletés sur les surfaces verticales (portes, plinthes, vitrages intérieurs).

Faites une analyse de détail, espace par espace, et dressez une liste des réparations ou opérations d'entretien nécessaires.

\section{Sols}

Que les sols de votre bibliothèque soient en bois, carrelage, moquette, linoleum, béton ou composites, ils doivent être propres, comme le reste de la bibliothèque. Pour cela, programmez périodiquement un nettoyage en profondeur avec votre service de 
ménage ou bien faites intervenir un spécialiste. Si votre moquette est trop sale pour pouvoir être récupérée, prévoyez dans votre budget les frais nécessaires à son remplacement par une moquette neuve ou par un autre revêtement moins sujet à l'usure.

Les sols doivent également répondre à des exigences de sécurité. Assurez-vous que les déplacements restent sécurisés même par temps froid ou humide afin que de simples aléas météorologiques ne transforment pas vos circulations, parvis, terrasses, escaliers et halls d'entrée en piscines ou en patinoires.

Le choix des matériaux a aussi des conséquences fonctionnelles, phoniques et esthétiques. Les surfaces dures comme le carrelage ont tendance à renforcer le bruit en renvoyant les ondes sonores tandis que la moquette aide à atténuer et à adoucir les sons produits dans une pièce.

\section{À vous de jouer}

Votre diagnostic a mis en évidence très peu de 0 et de 1 : marquez 25 points.

Vous avez environ $50 \%$ de 0 et de 1 ? 12 points.

Votre bâtiment a des problèmes importants, ou vous avez coché plus de $50 \%$ de 0 et de 1: accordez-vous entre 0 et 12 points.

Si vous utilisez l'annexe 1, reportez-y votre note CUTI sur 25.

\section{Passez à l'action : lancez une campagne de nettoyage et de petites réparations}

Ce diagnostic peut facilement aboutir à un plan d'action. Reprenez tous les 0 et 1 dans un document qui récapitule concrètement ce qui nécessite une intervention. Attaquez-vous en priorité aux espaces les plus visibles et les plus fréquentés (votre entrée principale et votre rez-de-chaussée) et travaillez à partir de ce point 
de départ en cercles concentriques. Assurez-vous que ces petits travaux soient bien financés, planifiés et effectués. L'été peut être propice à ce que votre bibliothèque se refasse chaque année une beauté.

\subsection{Le bâtiment de la bibliothèque n'est pas encombré inutilement}

Travailler quotidiennement dans un bâtiment conduit souvent à une certaine indulgence. Les gens s'habituent à leur environnement et se résignent à ce que les choses restent en l'état simplement parce qu'elles sont comme ça depuis longtemps.

L'un des détails facilement occultés est l'encombrement de l'espace. Un certain bric-à-brac a tendance à s'accumuler dans les bibliothèques: marque-pages, prospectus publicitaires, lettres d'information, écrans d'ordinateur, usuels relégués dans un coin et qui ne sont plus consultés, et pendant qu'on y est, étagères entières de livres non consultés, papier brouillon et stylos, câbles d'ordinateurs, flyers épinglés sur un tableau en liège... cette accumulation d'objets peut réellement créer une impression d'encombrement de vos espaces. Baigné au quotidien dans ce fatras, vous ne le remarquez même plus. Cette liste a pour but de vous rendre attentif à ces détails et de vous encourager à poser un regard critique sur l'état d'encombrement de votre bâtiment.

\section{Pourquoi c'est important}

\section{Encombrement à l'échelle macroscopique}

Il faut refuser toute forme d'encombrement. Contrairement à un lieu harmonieux et bien rangé, les espaces encombrés ne sont propices ni au repos ni à un séjour agréable. L'accumulation est le symptôme d'aménagements faits sans plan prémédité: les choses sont déplacées et ajoutées les unes aux autres sans que l'on prenne 
en compte l'impact que cela peut avoir sur le tableau d'ensemble. Sans adopter systématiquement l'esthétique minimaliste qui caractérise le design scandinave, il faut affirmer que la simplicité a sa place dans toute bibliothèque, quel que soit son style.

\section{Encombrement à l'échelle microscopique}

Pour en revenir à la simplicité, moins il y a de choses dans un système, plus il est facile de les ordonner et de leur donner un aspect impeccable. Prenez un exemple extrême et imaginez une collection de deux objets: il sera facile de les garder en ordre. Deux cents objets, ce sera plus difficile. Et 200000 ? VRAIMENT plus difficile.

Encore une fois, il ne s'agit pas seulement d'esthétique. Débarrasser votre bibliothèque du petit bazar qui l'encombre facilitera la vie de vos usagers et apportera de réels avantages au personnel.

Un exemple parmi d'autres: une bibliothèque propose des prospectus et des marque-pages sur un présentoir, près des postes informatiques publics. Il y a douze objets de communication sur ce présentoir, chacun d'un niveau différent d'actualité et de pertinence. Personne ne prend vraiment le temps de les lire (après tout, il y en a douze) et celui qui y jettera un simple coup d'œil a toutes les chances de passer à côté de l'information qui aurait pu l'intéresser. Un conseil? Divisez par deux le nombre de documents présentés. Il vous reste six documents? Recommencez jusqu'à ne garder que trois documents différents au maximum par présentoir.

Il y a des avantages évidents au fait de ne conserver que trois ou quatre prospectus: les usagers peuvent repérer d'un coup d'œil tous les titres et y trouver en un instant ce qui leur sera utile. Les plus maximalistes d'entre vous pourraient répondre que s'il y a seulement trois ou quatre objets, cela réduit les chances pour les usagers d'y trouver ce qui les intéresse. Effectivement, si le présentoir était dégarni au hasard, ce pourrait être le cas. Il faut faire confiance au personnel pour déterminer ce qui intéresse ou 
non les usagers (le mieux est que vos collègues fassent quelques enquêtes auprès du public pour le savoir!) et pour faire le tri dans cette collection de brochures. Bien fait, cet exercice augmentera la pertinence des documents laissés sur le présentoir en éliminant ceux dont personne ne se soucie. Ainsi, réduire l'encombrement ne se contente pas de rendre l'environnement plus agréable: la bibliothèque est aussi plus facile à utiliser.

\section{Autodiagnostic}

Prenez le temps d'ouvrir les yeux et de regarder vraiment votre bibliothèque. Il peut être utile de demander aux agents d'observer les secteurs où ils ne travaillent pas habituellement, de manière à porter sur ceux-ci un regard neuf. Prenez des notes. Les photographies sont un bon outil dans le cadre de cet exercice, car elles peuvent mettre en évidence les lieux trop encombrés. Rassemblez tout le monde, discutez de ce que chacun a vu. Envisagez, ici encore, de demander à des utilisateurs de vous aider à évaluer le degré d'encombrement de votre bâtiment. Il y a souvent, dans une bibliothèque normale, au moins une demidouzaine de recoins où s'entassent des choses inutiles.

\section{Les collections}

Si vous avez une politique de désherbage rigoureuse et régulière, c'est bien. Dans ce cas, vous savez déjà que les collections de la bibliothèque sont un candidat de premier choix pour une opération visant à désencombrer les lieux.

Si vous travaillez dans une petite bibliothèque sans beaucoup de moyens, et que vous n'y avez jamais fait beaucoup de désherbage, c'est le moment de vous lancer. Il ne s'agit pas nécessairement d'une lourde tâche: de la même façon que vous relevez les contenus non consultés sur votre site internet, vous devriez vous pencher sur les statistiques de circulation pour 
trouver les exemplaires qui ne sortent plus assez pour justifier la place qu'ils prennent sur les étagères. Conserver les classiques est une chose, mais s'accrocher à Windows 98 pour les nuls en est une autre... Mettez au pilon tout ce qui présente une couche de poussière.

Si vous travaillez dans une grande bibliothèque de recherche ayant pour mission de collecter et de conserver l'ensemble du savoir humain, vous pouvez sauter cette partie et passer directement au conseil suivant concernant le désencombrement de votre établissement. Mais même des bibliothécaires ayant travaillé pour de grandes bibliothèques de recherche savent que ce type de mission encyclopédique n'existe plus ou n'est plus réaliste. Si vous-même ou l'un de vos collègues êtes contrariés à l'idée d'éliminer des documents, c'est le moment de vous souvenir que l'espace est un bien rare, cher et non dématérialisable et qu'il représente la priorité numéro un. Si un document n'est pas du tout consulté, il faut le considérer sérieusement comme un candidat à l'élimination.

Essayez de prendre du recul. Jetez par exemple un œil sur les collections de référence imprimées. Si ces dernières ne sont pas plus réduites qu'il y a cinq ans, il est temps de les évaluer. Il se peut que la plupart de ces documents (si ce n'est la totalité) puissent être supprimés. Un autre domaine qui mérite d'être regardé de près est votre collection de magazines grand public. Beaucoup de titres perdent rapidement leur intérêt: pourquoi les laisser en libre accès alors que la date de péremption est passée? Le désordre s'installe si facilement dans les magazines. Le seul fait de retirer les vieux numéros de vos collections en libre accès va améliorer l'apparence de vos rayonnages.

\section{Les équipements}

L'une des règles incontournables de la bibliothèque est que si vous possédez des documents nécessitant l'usage d'un certain type 
d'équipements pour être consultés, vous devez alors également donner accès à ces équipements. Les microfilms et microfiches sont un bon exemple: si vous possédez de vieux journaux ou des thèses visibles uniquement sur ces supports, vous ne pouvez pas vous passer d'un lecteur de microfilms ou microfiches permettant à vos usagers de les lire. Il s'agit là simplement de bon sens. Mais lorsque vos collections changent et que vous commencez à vous débarrasser de certains formats, n'oubliez pas de réduire votre offre d'équipements en conséquence. Vous ne serez sans doute pas surpris d'apprendre que les bibliothèques passent souvent à côté de cette étape cruciale en gardant des équipements hérités du passé même si les collections proposées dans les formats correspondants décroissent en volume et en popularité. Ces quatre lecteurs de microfilms que votre bibliothèque a achetés en 1960 étaient probablement plébiscités à l'époque, mais, récemment, combien de fois ont-ils été utilisés tous les quatre simultanément? Il est peut-être temps d'en réduire le nombre. La même chose vaut pour les magnétoscopes, les lecteurs de CD ou DVD et tous les autres appareils qui ne rencontrent plus le succès d'autrefois.

\section{Les entrées du bâtiment}

Comment réagiriez-vous si quelqu'un venait chez vous et déposait des journaux gratuits, des horaires de bus ou de train, des flyers pour des manifestations culturelles et d'autres documents publicitaires juste devant votre porte? Vous seriez sûrement horrifié de ce fatras. Celui accumulé à l'entrée des bibliothèques fait exactement le même effet à la plupart des visiteurs. Faites en sorte que la première impression de ceux qui entrent dans la bibliothèque soit bonne en enlevant tout ça de vos sas et halls d'entrée.

Prospectus, brochures, lettres d'info et autres documents placés sur présentoirs

Les présentoirs accueillant des prospectus et d'autres documents de ce genre sont souvent victimes du même mécanisme 
d'expansion qui frappe les entrées de bâtiments: de plus en plus d'éléments s'y entassent, se faisant concurrence les uns les autres. Gardez surtout pour objectif d'être sélectif en proposant un choix restreint d'éléments pertinents et bien mis à jour.

\section{Programmation et événements}

Si de trop nombreux événements organisés par la bibliothèque n'encombrent pas son espace physique, ils peuvent en revanche encombrer son espace mental. Votre bibliothèque continue-t-elle d'accueillir par inertie une programmation au long cours plutôt que de faire le nécessaire pour provoquer une attention enthousiaste? Peut-être est-il temps de la revoir et d'arrêter certaines choses. Libérer du temps et des ressources financières peut vous permettre de tenter de nouvelles actions.

\section{Signalétique}

L'excès de signalétique peut, sans que vous vous en rendiez compte, créer un environnement fatigant, surtout si son aspect formel laisse à désirer. À l'avenir, plutôt qu'ajouter d'emblée un élément de signalétique, essayez de changer le contexte qui vous incite à le faire. Vous répondrez mieux aux besoins de vos usagers et l'aspect de votre espace en sera amélioré.

\section{À vous de jouer : estimez votre CUTI}

Cet autodiagnostic compte pour 20 points. Essayez de faire preuve d'objectivité et d'honnêteté dans votre jugement, même si cette estimation comporte bien sûr une part subjective.

Votre bibliothèque est un parangon d'ordre et de tranquillité? Accordez-vous 15 points ou davantage.

Votre bibliothèque est pleine à craquer? Restez sous la barre des 10 points. 


\section{Passez à l'action et réduisez l'encombrement}

Il y a deux étapes pour limiter le désordre et toutes deux peuvent être difficiles à mettre en œuvre.

Identifiez tout d'abord les facteurs qui en sont à l'origine. Vous devrez ensuite obtenir les consentements nécessaires pour supprimer des choses. Pour un présentoir à journaux, ce sera sans doute facile, mais d'autres zones comme les services ou les programmes culturels seront sûrement plus sensibles. Ces éléments sont au cœur des missions de la bibliothèque et auront probablement une forte dimension affective pour les bibliothécaires. Pour surmonter les conflits potentiels, faites passer votre discussion de la sphère des considérations subjectives à celle des besoins des usagers. Fiez-vous aux méthodes décrites dans les deux premiers chapitres de ce livre pour vous aider à déterminer ce qu'il convient de garder et ce dont il faut se débarrasser.

\subsection{Le mobilier répond aux besoins des usagers}

\section{Pourquoi c'est important}

\section{Difficulté $* *$}

Charles Eames, un designer du milieu du siècle dernier, disait que «le rôle du designer ressemble à celui d'un hôte attentionné qui emploie toute son énergie à essayer d'anticiper les besoins de ses invités». Si ce constat concernant l'hospitalité s'applique à tous les points de contact de la bibliothèque, il est évident qu'il vaut en particulier pour l'ameublement de votre bâtiment.

Le postulat de départ de ce diagnostic semble tout simple. Défendre le principe de meubles confortables va apparemment de soi: qui accepterait de proposer volontairement un mobilier 
inconfortable et inutilisable? Mais il ne s'agit pas seulement de s'assurer que chaises et fauteuils sont confortables; il faudra aussi vous demander s'ils sont fonctionnels et répondent à tous les besoins - ceux des utilisateurs comme les vôtres.

Amanda Etches et Aaron Schmidt racontent avoir eu un jour un échange avec le responsable d'une bibliothèque qui affirmait qu'il ne voulait pas "que les chaises soient trop confortables». Un passant surprenant cette conversation aurait pu être déconcerté. La suite de la conversation a éclairé ces propos: cette personne parlait spécifiquement de sièges pour les ordinateurs réservés à une consultation rapide (limitée à cinq minutes par consultation). Dans ce cas particulier, ce responsable appliquait en fait un des huit principes du Design UX exposés dans le chapitre 1 -il choisissait l'ameublement en fonction d'un type de comportement spécifique. En d'autres termes, si vous souhaitez que vos usagers ne passent pas plus de cinq minutes sur une tâche donnée en un lieu donné, n’y placez pas vos sièges les plus confortables, sachant que des sièges confortables vont leur donner envie de s'y attarder. L'environnement que vous créez influence le comportement des gens: anticipez l'impact de vos choix de manière avisée.

\section{Faites votre autodiagnostic}

Il est aisé de déterminer si le mobilier de votre bibliothèque est confortable: utilisez-le pendant une journée. Vous y sentez-vous bien? Avez-vous suffisamment de place pour votre ordinateur, vos papiers et votre tasse à café? Avez-vous mal au dos après être resté trente minutes à lire dans une chauffeuse? Répondre à des questions de ce genre devrait déjà vous éclairer. N'en restez pas là: demandez aux usagers de la bibliothèque ce qu'ils en pensent. Préparez une petite enquête ou bien interrogez-les pendant qu'ils utilisent le mobilier. 
Déterminer si un meuble est fonctionnel et répond aux besoins des utilisateurs peut être difficile, mais ce n'est pas impossible. Lorsque vous commencerez à réfléchir à l'utilité de votre ameublement, il sera essentiel de penser aux activités qu'il est censé faciliter. En voici quelques-unes:

$\odot$ étudier;

$\odot$ lire;

$\odot$ travailler en groupe;

$\odot$ utiliser un ordinateur portable;

$\odot$ utiliser un ordinateur fixe;

$\odot$ assister à des animations;

$\odot$ obtenir de l'aide à un bureau d'information;

$\odot$ emprunter des documents;

$\odot$ rendre des documents.

Envisagez de conduire quelques enquêtes contextuelles, d'observer les usagers lorsqu'ils se livrent à ces tâches. Les utilisateurs font-ils le tour de la bibliothèque à la recherche d'une place pour s'installer? Y a-t-il des groupes qui travaillent ensemble dans des espaces conçus pour un usage individuel? Des utilisateurs qui détournent les petites salles de groupe pour y travailler seuls? Les usagers déplacent-ils les meubles afin que ceux-ci s'adaptent mieux à leurs besoins? Les animations affichent-elles complet en permanence?

Ici aussi, vous pouvez poser des questions directement aux individus ou leur soumettre un questionnaire. Demandez aux usagers d'exprimer leur accord ou leur désaccord par rapport à des affirmations comme "Je trouve toujours une place à la bibliothèque ". Vous pouvez aussi proposer des phrases à compléter du type «La bibliothèque a besoin de plus de...». Cependant, ne 
justifiez pas un changement important de mobilier à partir des réponses apportées à ces phrases à compléter. Souvenez-vous que les opinions et le comportement des personnes sont souvent deux choses bien différentes. Relevez les réponses qui reviennent souvent et utilisez-les pour confirmer ou infirmer vos intuitions.

\section{À vous de jouer}

À l'issue de cette enquête par observation ou par questionnaire - le mieux étant de faire les deux-, accordez-vous jusqu’à 20 points.

Vous proposez des espaces confortables correspondant aux besoins de tous les types d'usagers: 20 points.

Votre mobilier présente des défauts importants ou se révèle inapproprié: 0 (si tout est à jeter) à 10 points.

Votre CUTI, si votre bibliothèque a déjà quelques années, sera sans doute compris entre 8 et 15 .

\section{Passez à l'action}

La seule façon d'améliorer du mobilier inconfortable est de le remplacer... par du mobilier confortable. Cette solution simple est malheureusement difficile à mettre en œuvre, du fait du prix élevé de l'ameublement. Si vous avez identifié des meubles inconfortables dans votre bibliothèque, faites une liste de priorités où figurent les éléments les plus utilisés. En les remplaçant en premier, votre action aura un impact important. Vous aurez sans doute besoin de demander des subventions pour terminer ce projet. Comme toujours, construisez votre argumentaire en vous appuyant sur les besoins des usagers. Utilisez les données que vous avez collectées pour présenter vos demandes et n'oubliez pas d'inclure quelques photographies de personnes contorsionnées, assises dans des positions inconfortables. Il est 
difficile d'en dire plus ici pour évaluer le caractère fonctionnel de votre mobilier sans connaître vos exigences, vos besoins et vos contraintes. Voici cependant trois conseils:

Appuyez-vous sur ce que font les usagers. S'ils sont sans cesse en train de réagencer les meubles de la même façon, observezles et essayez d'introduire des changements en accord avec ces réorganisations.

Si vos problèmes vous conduisent à acquérir différents types de mobilier, achetez des meubles qui pourront facilement être réutilisés dans des buts différents et pour des besoins qui évoluent. Pensez à la manière dont votre bibliothèque est susceptible de se transformer dans les cinq prochaines années, et assurez-vous que vos nouveaux meubles pourront s'y adapter.

Opérez des choix délibérés quand vous prenez la décision d'acheter et de mettre en place des meubles dans votre bibliothèque. Des meubles différents incitent à des usages différents: il est donc nécessaire de prendre en compte ces usages au moment de l'achat et du choix des emplacements. Le point suivant développe cet aspect.

\subsection{Le bâtiment s’adapte à des comportements variés}

\section{Pourquoi c'est important}

\section{Difficulté $* *$}

Les bibliothèques sont utilisées par des personnes très différentes pour des raisons différentes. L'une d'entre elles pourra rechercher, par exemple, un espace calme où utiliser son ordinateur portable, tandis que d'autres pourront avoir besoin d'un espace pour travailler en groupe. Chacune de ces activités est légitime et chacune exige un environnement différent. Comment 
faire en sorte que tous les usagers soient confortablement installés et puissent mener à bien leurs activités, en particulier quand certaines de ces activités sont incompatibles entre elles? Une solution réside dans la mise en place de différentes zones adaptées aux différents usages.

Appliquer le principe du zonage à votre bibliothèque est une façon coopérative de gérer les comportements. Les tentatives pour faire respecter des règlements d'une rigueur déraisonnable créent un climat de conflit entre la bibliothèque et ses usagers et sont bien rarement couronnées de succès. Il est clair que les bibliothécaires préfèrent occuper leurs journées de façon plus productive qu'en faisant la police.

\section{Où en est votre bibliothèque?}

Si votre équipe reçoit beaucoup de plaintes au sujet de certains comportements comme le bruit ou la consommation de nourriture, cela indique que les besoins de différents usagers sont en conflit. Pour évaluer réellement la situation, passez un peu de temps à observer les utilisateurs de votre bâtiment. Vous pouvez par exemple rechercher les activités en concurrence qui se déroulent en un même lieu -comme des personnes essayant de travailler en groupe et individuellement.

Choisissez une période de deux semaines pour conduire votre enquête contextuelle. À la fin des deux semaines, donnez-vous 20 points si les activités de vos usagers se déroulent la plupart du temps harmonieusement, 15 points si vous observez quelques conflits et 0 point si vous observez beaucoup de conflits.

\section{Passez à l'action}

\section{et adaptez les lieux aux différents comportements}

Si votre bibliothèque a eu un mauvais résultat à cet autodiagnostic, il est recommandé d'établir un zonage permettant de 
différencier les types d'activités. Le projet peut sembler a priori coûteux, mais ne l'est pas nécessairement. En fait, le zonage se met parfois en place naturellement parce que les différentes sections se trouvent en général dans différentes parties du bâtiment de la bibliothèque. Vous ne le réalisez peut-être pas, mais beaucoup de bibliothèques (peut-être même la vôtre) possèdent déjà une expérience en matière de création d'espaces dédiés à des usages différenciés: beaucoup d'entre elles ont un espace dédié aux pratiques collectives, heure du conte ou ateliers pratiques; certaines bibliothèques ont des espaces de lecture où même taper sur un clavier d'ordinateur est interdit - et a fortiori le fait de parler. Tirez parti de cette expérience pour vous assurer que vos usagers peuvent accomplir leurs tâches dans toute leur diversité. Un autre élément à garder en tête est que le type de mobilier et sa mise en place dans l'espace peuvent avoir un impact sur la capacité de vos zones à bien fonctionner. Vous venez d'entendre parler de l'importance d'avoir un mobilier qui correspond aux besoins des usagers. Vous devez aussi prendre en compte l'impact de l'ameublement sur votre projet de service. Par exemple, si vous voulez dédier un espace:

$\odot$ aux activités silencieuses, vous choisirez probablement des carrels de travail individuels et des chaises de bureau;

$\odot$ au travail en groupe, vous devrez probablement opter pour de grandes tables et des chaises;

$\odot$ aux échanges informels, des canapés et des tables basses enverront aux usagers un signal adéquat.

Il existe bien sûr d'innombrables possibilités en dehors de celles qui viennent d'être évoquées. Gardez à l'esprit que l'ameublement que vous choisissez pour un espace donne des indications tacites sur l'usage qui peut en être fait. Faites des choix conscients quand vous prenez la décision d'acheter du mobilier pour une zone donnée. 
Une fois que vous aurez délimité des zones dans votre bâtiment, assurez-vous d'utiliser d'autres moyens en plus de l'ameublement, pour signifier aux gens quelles sont ces zones et quel usage est autorisé dans chacune d'entre elles. La solution retenue par les bibliothèques de l'université de Houston envoie un message clair dans une typographie facile à lire.

Si votre bibliothèque est petite, la mise en place de zones sera plus délicate: dans un petit bâtiment, le bruit venant d'un espace peut se propager partout. Pour créer un zonage, il est possible de prévoir différents usages à différents moments de la journée ou de la semaine. Si votre bibliothèque est de petite taille, vous le faites sans doute déjà par défaut. Vous vous attendez à ce que l'heure du conte et les heures de sortie d'école soient un peu agitées, alors que les soirées seront peut-être un peu plus calmes. $\mathrm{Au}$ lieu d'un zonage des espaces, envisagez un zonage des activités fondé sur le temps.

\section{5 Les usagers ont facilement accès aux prises de courant}

\section{Pourquoi c'est important}

\section{Difficulté *}

Si vous avez déjà fait le tour d'une rame de train, d'une gare ou d'une salle de réunion à la recherche d'une prise de courant, pour en trouver une finalement hors d'usage ou déjà utilisée, il n'y a pas besoin de vous expliquer les enjeux. C'est frustrant, n'est-ce pas? Épargnez cette frustration à vos usagers en faisant en sorte que tous ceux qui le souhaitent soient en mesure de brancher un équipement. Voire deux.

\section{Évaluez le besoin en prises électriques}

Il n'existe pas de normes ou de recommandations systématiques sur le nombre de prises électriques dont vous avez besoin, parce 


\section{Library Learning Zones}

\section{Check out our new Zone Locations ${ }^{\bullet}$} Starting March 21 st!

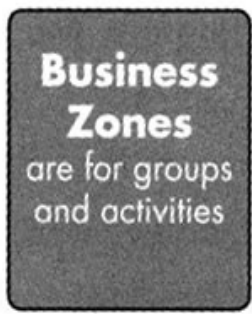

- For groups and activities

- Conversation and group study permitted

- No restriction on cell phone usoge

- Area may be noisy

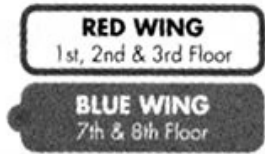

BROWN WING

None

GOLD WING

Ist \& 2nd floor

Quiet

Zones are for smaller groups and casual study
- for small groups and casual study

- Conversations should be whispered

- Small group study (2.4) is permitted

- Set cell phones to vibrate or silent; please leave areo to take phone calls

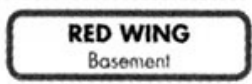

BLUE WING

2nd Floor

BROWN WING

None

GOLD WING

3rd Floor \& Bosement

RED WING

- For individual study

with no interruptions

- No conversations permitted

- No group study permitted in these oreos

- Set cell phones to vibrote or silent; please leave area to take phone colls
Regent's Reoding Room

BLUE WING

3rd, 4th, 5th \& 6th Floor

BROWN WING

2nd, 3id, 4th \& 5 th Floor

GOLD WING

None

\section{Pick the zone that's right for you!}

\section{UNIVERSITY of HOUSTON LIBRARIES}

Figure 3.1 Signalétique indiquant le zonage des espaces dans les bibliothèques de l'université de Houston. 


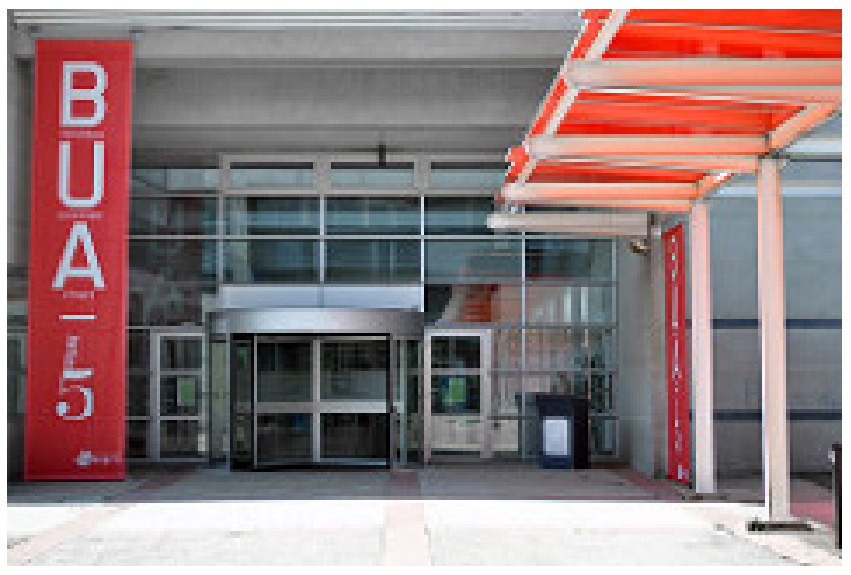

Figure 3.2 Une adaptation en France, à la bibliothèque Belle Beille de l'université d'Angers, exploite la division du bâtiment en trois blocs et une signalétique colorée sur les poteaux porteurs du bâtiment.

que les besoins des uns et des autres sont évidemment différents. Ils dépendent de la taille de votre bibliothèque, du nombre et du type d'usagers, de la capacité d'accueil de votre bâtiment, de votre infrastructure électrique et d'un ensemble de données locales spécifiques à votre établissement. Pour cette raison, voici quelques conseils sur la manière de déterminer vous-même ce nombre, plutôt que de proposer un nombre arbitraire.

Consacrez un peu de temps à observer les personnes qui fréquentent votre bibliothèque. Confiez cette tâche à quelques collègues et soyez attentif au nombre de personnes qui utilisent les prises à différents moments de la journée et à différentes périodes de l'année. C'est aussi une bonne occasion d'interroger les usagers sur la question. Plutôt que de rédiger et de distribuer une enquête formalisée, discutez avec des personnes que vous voyez utiliser des appareils électroniques dans la bibliothèque. Demandez-leur à quelle fréquence ils utilisent ces équipements dans la bibliothèque et ont besoin de les recharger, s'ils ont déjà eu du mal à trouver une prise... Cela vous permettra d'évaluer s'il est nécessaire d'en ajouter et combien. 
Si vous proposez suffisamment de prises à vos usagers, accordezvous 15 points.

Si les usagers attendent leur tour pour se brancher ou apportent leurs multiprises, 0 point.

De nombreuses bibliothèques se situent autour d'une moyenne de 7 points.

\section{Passez à l'action et proposez plus de prises}

La plupart des bibliothèques ont été construites avant la généralisation des appareils électroniques: elles n’ont pas été conçues pour recevoir des personnes qui utilisent et chargent ordinateurs portables, téléphones et/ou tablettes. Votre bâtiment répond peut-être déjà aux besoins en matière de prises, mais si vous remarquez que des usagers ou des membres de l'équipe doivent avoir recours à des stratégies élaborées pour accéder à l'électricité, à base de rallonges, de multiprises ou de déplacement de mobilier, ou si vous avez eu des réclamations de personnes demandant plus de prises, prenez-le en compte.

\section{Ajoutez des prises}

Si votre bâtiment manque de prises, vous pourrez en ajouter quelques-unes. Cela va exiger l'intervention d'un professionnel, mais, en fonction des installations électriques de votre bâtiment, cela ne coûtera pas nécessairement très cher. Attention, dans un ERP de type S (Établissement recevant du public), cela doit toujours être certifié par un contrôleur technique. Sollicitez d'abord les services techniques de votre établissement, puis faites réaliser quelques devis pour vérifier la faisabilité du projet. Une solution très souple, peu connue en France, consiste à installer au plafond une grille de type "canalis », d'où partent des prises munies de rallonges sous la forme de câbles rétractables ou spiralés. Ce type de rallonges peut être utilisé pour alimenter une 
rangée d'ordinateurs ou de multiprises. L'avantage de cet aménagement est que l'ameublement de la bibliothèque peut être configuré sans avoir à prendre en compte l'emplacement des prises puisque ces dernières peuvent être mises à disposition presque partout. C'est la solution adoptée dans la plupart des grandes surfaces, qui reste encore trop peu connue et encore moins utilisée par les services techniques des collectivités publiques.

\section{De nouveaux meubles}

Si votre infrastructure actuelle le permet, vous pouvez acheter des tables et des chaises qui offriront un accès aisé aux prises de courant. Il s'agit là d'une solution coûteuse, mais conviviale et confortable.

\section{Multiprises}

Créer davantage de prises peut passer simplement par la mise en place de quelques multiprises installées à des emplacements stratégiques. Il vous revient de vous assurer au préalable que le matériel mis à disposition répond bien aux normes NF, que les prises choisies supportent la puissance et ont la protection nécessaire au rechargement simultané de plusieurs équipements, en envisageant les plus gourmands que l'on est susceptible d'y brancher. Ayez à l'esprit la multiplication des vidéoprojecteurs, qui exigent souvent une puissance importante.

\section{Stations de rechargement}

Parfois, les gens ont seulement besoin d'un endroit où recharger la batterie de leurs appareils pendant qu'ils se livrent à d'autres activités dans le bâtiment. Il existe, dans certains aéroports, des stations de recharge ergonomiques offrant dans un petit espace une multitude de prises alignées dans lesquelles il est possible de mettre en charge un nombre important d'appareils, voire des stations proposant des connecteurs et des alimentations 
correspondant aux types de chargeurs les plus courants. Si le fait de laisser des appareils sans surveillance pose problème, il existe des stations de rechargement où il suffit de laisser son appareil dans un casier qui ferme à clé.

En ajoutant des prises supplémentaires, vous risquez de provoquer un enchevêtrement de câbles. Assurez-vous donc que la solution retenue ne crée pas de problème esthétique. Il existe toute une gamme de produits permettant de contourner le problème: les catalogues de Legrand, Merlin Gerin, Schneider Electric ou GE General Electric vous donneront plein d'idées d'équipements aux normes françaises conjuguant esthétique et sécurité. Certains fabricants de mobilier commercialisent également des équipements ingénieux.

\subsection{Plus de lectures}

1. Designing Libraries. [En ligne] < http://www.designinglibraries. org.uk/>.

2. Valotteau H., Couleurs en bibliothèque: architecture, signalétique, esthétique, Mémoire d'étude de conservateur des bibliothèques, sous la direction de Christophe Catanèse: Villeurbanne, Enssib, 2011. 


\section{Chapitre 4. Les espaces d'accueil}

À l'origine, les espaces d'accueil devaient être traités en deux chapitres, l'un consacré aux services de référence (anglicisme évoquant le renseignement bibliographique) et l'autre aux services de prêt/retour. Cette distinction est le reflet d'une organisation catégorielle ancienne, fondée sur les statuts et les missions des personnels et rien d'autre. Bref, une vision des espaces de service et d'accueil qui est tout sauf centrée sur l'utilisateur et qui ne reflète en rien la manière dont les gens voient et utilisent ces services.

Finalement, un seul et même chapitre regroupe tous les diagnostics qui concernent les espaces d'accueil. Si vous voulez que vos institutions soient vraiment centrées sur leurs utilisateurs, il faut arrêter de les organiser en fonction des silos traditionnels, et plutôt les concevoir en tenant compte de la façon dont les gens les utilisent.

Ce chapitre vous propose de faire des autodiagnostics sur tous les types d'accueil. La plupart des bibliothèques en possèdent un ou plus: ces espaces sont souvent des zones chaudes, de forte activité au sein des bâtiments, ce qui les rend très importants. Comme vous allez le constater, les points traités ici, allant du plus simple au plus complexe, ont tous un niveau de difficulté élevé: faire évoluer ces espaces d'accueil n'est pas chose facile, que les changements soient d'ordre matériel ou conceptuel.

\subsection{Les usagers s'approchent facilement des bureaux d'accueil}

\section{Difficulté $* *$}

À quoi ressemblent vos bureaux d'accueil? Les gens s'en approchent-ils facilement lorsqu'ils cherchent de l'aide ou 
sont-ils au contraire hésitants et s'excusent-ils de vous demander quelque chose? Dans cette partie, vous entendrez parler des proportions de vos bureaux ou banques d'accueil ainsi que de quelques idées pour les rendre plus confortables et plus accessibles aux utilisateurs.

\section{Pourquoi c'est important}

Quand vous aurez lu le chapitre 5, consacré aux règlements et services aux utilisateurs, et fait les exercices proposés, vous saurez ce qu'est exactement une philosophie de service et comment en doter votre établissement. Sans attendre que vous en soyez arrivé à ce stade de votre lecture et pour résumer, on entend par là les valeurs et l'éthique promues par la bibliothèque en termes de relations avec son public. Il y a fort à parier qu'une telle philosophie contiendra quelque chose sur la bienveillance, l'ouverture d'esprit, l'écoute et l'accessibilité. Regardez maintenant les bureaux d'accueil de votre bibliothèque. Ces espaces transmettent-ils ce genre de message? Sont-ils en accord avec ce type de valeurs? Vos bureaux d'accueil sont-ils conviviaux, ouverts et accessibles? Si c'est le cas, bravo! Vous pouvez passer directement au point suivant. Sinon, continuez votre lecture.

L'époque des bureaux d'accueil surélevés, massifs et imposants est révolue. Ces guichets d'accueil monumentaux intimident la plupart des utilisateurs. Si vous avez déjà essayé d'y accueillir le public au cours de votre vie professionnelle, vous savez que vos sourires et tentatives de contact visuel n'y changent rien. Il y a de grandes chances pour que les gens continuent à s'excuser de vous déranger avant de poser leurs questions, parce que l'énorme bureau derrière lequel vous êtes assis n'est pas perçu comme accueillant et accessible. Il est temps de changer cela.

Le plus souvent, la perception d'un objet est différente d'un individu à l'autre. Il est possible qu'en regardant le bureau d'accueil 
de votre espace jeunesse, vous trouviez qu'il est le plus agréable, le mieux pensé et le plus accessible du monde... Et pourtant, mettez un enfant de 4 ans face à ce même bureau, il s'enfuira en pleurant!

\section{À vous de jouer : accessible ou intimidant?}

Le meilleur moyen d'évaluer l'accessibilité, c'est d'interroger les personnes qui comptent le plus, celles qui doivent apprécier et utiliser votre bureau d'accueil. Non, pas les bibliothécaires, les utilisateurs et utilisatrices. Pour évaluer leur ressenti concernant le bureau d'accueil de votre bibliothèque, faites quelques exercices.

Le premier est emprunté au monde du design participatif. Placez un tableau blanc devant votre bureau d'accueil et écrivez-y la question suivante: «Trouvez-vous que le bureau d'accueil a l'air accessible et convivial?» Dessinez deux colonnes sous la question, l'une pour les «oui» et l'autre pour les «non». Mettez un feutre à disposition des utilisateurs pour qu'ils mettent une croix dans l'une des deux colonnes et encouragez-les à voter.

S'ils votent majoritairement pour le oui: 10 points.

Si c'est le non qui l'emporte: 0 point.

Puisque le premier exercice s'appuie uniquement sur les opinions des utilisateurs, ce second va vous aider à équilibrer vos résultats en introduisant l'étude des comportements. Pour cet exercice, demandez à votre équipe de recenser le nombre total d'interactions avec le public au bureau d'accueil pendant une journée et de noter toutes celles qui commencent par des excuses. Vous les avez déjà entendues, ces demandes du type: «Excusezmoi de vous déranger, mais puis-je vous poser une question? » ou «Je ne suis pas sûr que vous pourrez me répondre, mais j'ai une question à vous poser». 
Si vous obtenez plus de questions qui commencent sans excuses: 15 points.

Si vous obtenez une majorité de questions qui commencent par des excuses: 0 point.

Additionnez les deux CUTI et reportez-les dans le tableau proposé en annexe. Le CUTI maximum pour ce diagnostic est de 25 points.

\section{Passez à l'action et devenez plus accessible}

Si, après les deux exercices précédents, votre score est bas, vous allez vouloir faire évoluer votre bureau ou banque d'accueil. Voici quelques idées pour y parvenir, classées de la plus simple à la plus complexe.

\section{Sortez de derrière le monolithe}

Cela fait des années que l'on parle de renseignement mobile, de bibliothécaires en mouvement dans les espaces publics. Une manière facile d'éliminer la contrainte d'un bureau d'accueil trop imposant, sans avoir à dépenser beaucoup pour le démanteler, c'est tout simplement de sortir de derrière le bureau pour accueillir le public. Il y a fort à parier que les gens ne remarqueront plus l'énorme banque d'accueil placée derrière un bibliothécaire souriant et accessible.

\section{Supprimez les obstacles}

Regardez votre bureau d'accueil. Y a-t-il un comptoir, une estrade, un écran d'ordinateur, des obstacles entre les professionnels qui y travaillent et les utilisateurs? Si oui, enlevezles. Démontez le comptoir et retirez l'estrade. Souvent, ces éléments étaient prévus pour cacher les câbles informatiques, mais il existe aujourd'hui d'autres solutions pour les dissimuler (périphériques sans fil, système de gestion de câblage, etc.). 


\section{Réduisez la voilure}

Si vous pouvez supprimer un ou deux éléments mobiliers pour réduire la taille de votre bureau d'accueil, faites-le. On l'a déjà dit, les bureaux d'accueil massifs sont souvent intimidants, en grande partie à cause de leur taille. Réduire leurs dimensions fera des miracles.

Si vous avez la chance d'avoir un budget suffisant pour réaliser une rénovation complète, vous pouvez démonter votre banque d'accueil et installer quelque chose de plus léger.

La plupart des principes décrits dans cet ouvrage vous y aideront, ainsi que quelques références documentaires sur le design de service (Service Design). Un peu de travail de terrain vous sera également très utile. Visitez d'autres établissements (lisez: autres que des bibliothèques) pour observer comment les bureaux d'accueil y sont pensés. Il y a beaucoup à apprendre - en bien, comme en mal- du design de service pratiqué dans d'autres services publics, dans des espaces de vente, des banques ou dans les espaces d'accueil des hôpitaux, par exemple. Ne négligez aucun détail. L'un de nos préférés (pour le design de service et le modèle dans son ensemble) peut être observé dans l'Apple Store le plus proche de chez vous. Le concept de Genius Bar d'Apple pourrait aisément être transposé en bibliothèque et faire des merveilles dans la vôtre. Pensez également aux boutiques de téléphonie mobile, présentes dans la plupart des villes.

\subsection{Les besoins changent, les bureaux d'accueil aussi}

Avez-vous, chez vous, un endroit où vous aimez particulièrement vous installer? Tout le monde a un coin confortable de ce genre. Il n'est pas toujours le même selon le moment de la journée, le temps qu'il fait ou la saison, ou selon votre occupation 
du moment - travailler sur ordinateur portable ou lire un livre, par exemple. Parfois, votre coin favori peut être trop froid ou pas assez ensoleillé, ou trop distrayant pour le travail que vous souhaitez accomplir. Du coup, vous vous déplacez et trouvez un autre endroit, plus adapté à ce que vous faites. Ne serait-il pas merveilleux de pouvoir faire la même chose avec les bureaux d'accueil?

\section{Pourquoi c'est important}

Il existe de nombreux arguments en faveur d'une évolution des bureaux d'accueil.

Un exemple: si votre bibliothèque a un bureau de renseignement, vous y traitez sans doute toutes sortes de demandes, de la simple question d'orientation dans la bibliothèque à des interrogations bibliographiques complexes.

Les bureaux d'accueil en position debout sont parfaitement adaptés aux échanges d'informations rapides, mais pas du tout aux rendez-vous bibliographiques. Autre point: si vous avez déjà renseigné un utilisateur en fauteuil roulant depuis un bureau haut, vous savez que si vous pouviez ajuster la hauteur de votre surface de travail, l'interaction avec lui s'en trouverait grandement améliorée comme son confort et le vôtre.

Si vous avez récemment commandé du mobilier pour votre bibliothèque, ou si vous avez parcouru avec envie un catalogue de mobilier, en rêvant d'obtenir un budget d'investissement, vous avez remarqué la variété de l'offre de mobilier à usage commercial proposée de nos jours. Ces meubles sont pour la plupart modulables et faciles à assembler, vous permettant ainsi de les modifier aisément en enlevant ou en ajoutant des éléments souvent mobiles, et prééquipés pour faciliter l'électrification et l'installation d'éclairages, rendant votre bureau d'accueil réellement reconfigurable et même déplaçable. Ainsi, si vous ne voulez plus 
que le soleil vous aveugle l'après-midi, vous pouvez prendre votre bureau et le déplacer (peut-être même le faire rouler) pour l'installer à un endroit plus approprié à quelques pas de là.

\section{À vous de jouer: To be or not to be flexible?}

Évaluer la possibilité de modifier vos bureaux d'accueil ne vous prendra qu'un instant: soit ils sont modulables, soit ils ne le sont pas. Toutefois, selon leur localisation et leur implantation dans la bibliothèque, vous vous rendrez compte qu'il existe plusieurs niveaux de flexibilité et que votre bureau d'accueil n'est pas forcément aussi adaptable que vous le souhaiteriez. Pour vraiment évaluer cela, procédez à l'analyse de ses points faibles. Demandez aux agents qui travaillent à l'accueil d'en dresser la liste et, une fois cette liste en votre possession, recensez tout ce qui peut être changé sans effort (c'est-à-dire en un minimum de temps et sans dépense):

$\odot$ si plus de la moitié des points listés peuvent être modifiés sans effort: 25 points;

$\odot$ si c'est moins de la moitié: 12 points;

$\odot$ si aucun des éléments de la liste ne peut être modifié rapidement et à bas coût: 0 point.

\section{Passez à l'action pour accroître la flexibilité de votre bureau d'accueil}

Si votre bibliothèque a fait un petit score au dernier diagnostic, vous devriez faire contre mauvaise fortune bon cœur, vous débarrasser du bureau d'accueil actuel et en acheter un nouveau. Les idées présentées dans cette partie peuvent vous aider à choisir un nouveau mobilier. Si votre score était moyen, et que vous avez la possibilité d'effectuer des changements rapidement, ces principes peuvent vous guider. 


\section{Les agents sont impliqués dans l'aménagement de l'accueil}

Impliquer les équipes tout au long du processus d'aménagement de l'accueil est très utile: faites-le du début à la fin, de la phase de conception à l'installation et même au-delà, jusqu'au moment où vous évaluerez comment fonctionne le bureau d'accueil, après quelques semaines de mise en service. Le risque de vous retrouver avec un mobilier non fonctionnel et inadapté aux besoins des agents est réduit lorsque ces derniers sont associés au processus dès l'origine.

\section{Les bureaux d'accueil ne sont pas fixés au sol}

Ce que vous pouvez faire très facilement pour améliorer la flexibilité de votre bureau d'accueil est de ne pas le fixer au sol. Même si vous ne comptez pas le déplacer tous les jours, ne pas le fixer au sol vous offrira la possibilité d'effectuer des changements rapidement et à moindre coût.

\section{L'ergonomie des bureaux d'accueil s'adapte aux besoins des équipes et des utilisateurs}

Des ergonomes professionnels peuvent réaliser un audit de votre bureau d'accueil. Si vous travaillez en bibliothèque universitaire, il est possible que des ergonomes exercent sur le campus. Si ce n'est pas le cas, le service hygiène et sécurité ou de médecine du travail de votre établissement peut vous orienter vers le service approprié pour faire une «étude de poste de travail». Faire réaliser un audit de votre bureau d'accueil pour qu'il fonctionne de manière optimale, à la fois pour vos équipes et vos utilisateurs, est une excellente idée. Une évaluation par un professionnel vous indiquera précisément le niveau de flexibilité que cet équipement doit atteindre pour s'adapter aux besoins de chacun. Un bureau et un siège ajustable en hauteur sont une priorité; pas seulement pour permettre au personnel de se tenir debout ou 
de s'asseoir au bureau, mais aussi pour pouvoir ajuster la hauteur du bureau et optimiser le confort des utilisateurs, ce qui se révèle particulièrement utile pour une personne en fauteuil roulant, par exemple.

Une disposition adaptée du lecteur de code-barres, du système de lecture / écriture des antivols, de l'écran, du clavier, de la souris, permettant de limiter les efforts et les gestes inutiles et garantissant la disponibilité des personnes chargées des interactions avec le public, a aussi son importance.

\section{Les bureaux d'accueil sont modulables}

La modularité est un mot à la mode dans le jargon des vendeurs de mobilier. En réalité, ce que vous permet la modularité, c'est de reconfigurer vos bureaux d'accueil selon vos envies ou vos besoins. La plupart des bureaux d'accueil modulables sont composés d'éléments qu'il est possible d'ajouter ou d'enlever selon la situation, que ces éléments soient des plans de travail, des tiroirs, des cloisons de confidentialité ou toute autre chose à laquelle vous pouvez penser. Bien sûr, vous n'aurez sans doute pas à faire ce genre de changement au jour le jour. Pourtant, la modularité est plus rentable sur le long terme, car elle vous permet d'acquérir des éléments supplémentaires ou d'en enlever selon l'évolution de votre service ou de vos besoins. Le contraire d'un équipement modulable est la banque d'accueil sur mesure (ou pire, dessinée par l'architecte) qui ne souffre aucune modification: un choix assurément coûteux, que plus personne ne devrait faire.

\section{Les bureaux d'accueil vous permettent de reconfigurer l'espace sans effort}

Il existe des bibliothèques qui ont transformé tout un niveau en un gigantesque amphithéâtre ou en salle de cinéma... voire en piste de danse. D’autres ont accueilli les médias locaux pour 
couvrir des événements importants. Certaines ont été utilisées comme abris pendant des catastrophes naturelles ou d'autres crises. Il existe aussi des bibliothèques qui ont transformé leurs halls en espaces de réception à l'occasion de cérémonies officielles. Aucun de ces usages imprévus des bibliothèques n'aurait été concevable si des bureaux d'accueil inamovibles et monolithiques avaient trôné en plein milieu de tous les espaces. Tout ce que l'on peut dire, c'est qu'il est judicieux de concevoir, d'acheter ou de construire des bureaux d'accueil qui puissent être déplacés avec un minimum d'efforts si nécessaire.

\subsection{Les gens reçoivent de l'aide quand et où ils en ont besoin}

\section{Difficulté $* * *$}

Il y a quelques années, on pouvait voir aux États-Unis une excellente publicité télévisée pour une enseigne de bricolage. Elle montrait un client perplexe et esseulé parcourir les allées d'un magasin concurrent et chercher désespérément un vendeur pour trouver son chemin. Finalement, le client repérait un vendeur au bout d'une très longue allée, et lançait d'une voix implorante: «Excusez-moi!» L'employé faisait volte-face en regardant le client d'un air penaud, avant de prendre la fuite dans la direction opposée.

Depuis sa diffusion, on imagine, malgré soi, les employés prenant leurs jambes à leur cou quand un client entre dans leur magasin.

La bonne nouvelle pour les bibliothèques, c'est qu'il est très rare de voir un bibliothécaire fuir à l'arrivée du public. La mauvaise, c'est qu'on rencontre partout un problème fondamental, qui se pose à toutes les entreprises de services: aider les usagers où ils en ont besoin et quand ils en ont besoin. 


\section{Pourquoi c'est important}

Un quiz rapide:

Première question: Quel est le contraire de la formule «où et quand ils en ont besoin»? Si vous répondez « où et quand nous le fournissons», vous avez tout bon.

Question suivante: laquelle des deux propositions est centrée sur l'utilisateur et laquelle est centrée sur la bibliothèque? Oui, c'est une question purement rhétorique; non, vous ne trouverez pas la réponse écrite à l'envers au bas de la page.

Même si vous considérez que votre bibliothèque est centrée sur ses utilisateurs, vous continuez peut-être à concevoir vos services d'une manière qui est plus pratique pour vous que pour le public. Un exemple classique, plus fréquent qu'il n’y paraît, est le cas d'une bibliothèque qui décide d'offrir un service de réponse en ligne en temps réel, mais seulement pendant quelques heures, en milieu de journée, parce que c'est le moment le plus commode pour trouver du personnel disponible, et non parce que c'est le moment où les utilisateurs en ont besoin ou peuvent y faire appel.

D'un côté, ces bibliothèques ont fait le louable effort de faire un pas dans la bonne direction - connaître suffisamment leur communauté pour savoir que des personnes ont besoin de ce nouveau service. De l'autre, il est évident qu'elles ne sont pas allées assez loin dans cette direction - connaître les besoins de chacun est important, mais leur offrir un service sans tenir compte de leur disponibilité est absurde.

Pensez au nombre de bureaux d'accueil que compte votre bibliothèque. Si vous en avez plus d'un, est-ce parce que différents départements veulent avoir le leur pour rendre le même type de services ou bien le faites-vous parce que cela a du sens et est plus utile pour vos usagers? Le premier cas est fréquent: répondez avec franchise pour votre bibliothèque. 


\section{À vous de jouer}

La meilleure manière de faire cet autodiagnostic est de mettre en place une enquête toute simple à la sortie du bâtiment et sur votre site internet. N'oubliez pas qu'aider vos utilisateurs où et quand ils en ont besoin est aussi valable en ligne que dans la bibliothèque.

Les deux versions peuvent se décliner autour de ces deux questions:

1/ Avez-vous eu besoin d'aide pour trouver ce que vous cherchiez aujourd'hui? (Oui / Non)

2/ Si oui, avez-vous eu des difficultés à trouver la bonne personne pour vous aider lorsque vous en aviez besoin? (Oui / Non)

Si les utilisateurs interrogés répondent plus souvent «Non » que «Oui» à la question 2: 25 points.

Si les réponses sont également partagées entre «Oui» et «Non»: 12 points.

Si vos membres choisissent plus «Oui» que «Non» à la question 2: 0 point.

\section{Passez à l'action : Renversez les priorités, moins de «vous " et plus de «eux»}

Si votre bibliothèque n'a pas obtenu le score maximal, voici quelques idées pour vous permettre d'améliorer vos performances et de fournir une aide véritablement centrée sur l'utilisateur.

Évaluez avant tout

Avant d'opérer le moindre changement dans votre offre de services qui réponde à l'objectif d'être plus centré sur l'utilisateur, mieux vaut d'abord jouer la carte de l'évaluation. Utilisez les 
résultats de l'enquête réalisée à la sortie de votre bibliothèque comme point de départ d'une réflexion approfondie sur votre offre de services. Qu'ont donné les résultats de l'enquête in situ par rapport à ceux de l'enquête en ligne? Vos visiteurs ont-ils plus de facilité dans la bibliothèque qu'en ligne ou vice versa? Utilisez ces données pour vous aider à vous concentrer sur votre point le plus faible. Une fois ce point identifié, pourquoi ne pas investir un peu de temps et d'efforts dans une enquête directe auprès de vos usagers?

Pour toucher au cœur des besoins de vos usagers, optez pour une méthode fondée sur l'analyse des attitudes, comme l'entretien avec des utilisateurs. Si vous avez le temps, essayez aussi de lancer des sondes culturelles, cette forme d'enquête ethnographique allégée présentée au chapitre 2 , pour mieux saisir quelle est la place de la bibliothèque et de ses services dans la vie des membres de votre communauté. On ne dira jamais assez que, pour développer des services réellement adaptés aux attentes du public, il faut commencer par bien comprendre les besoins des individus.

\section{Combinez}

Quel que soit le type de bibliothèque, publique ou universitaire, l'une des sources de confusion les plus répandues est le syndrome du «Où dois-je aller demander?». L'utilisateur ne sait à quel bureau s'adresser en fonction de ce qu'il cherche. C'est pourquoi la question 2 de l'enquête disposée à la sortie de votre établissement demande si les usagers ont trouvé la «bonne personne» (et pas seulement "quelqu'un»). Une bonne raison à cela: ils n'auront sans doute pas de difficulté à trouver quelqu'un pour les aider quand ils le souhaitent, mais ils auront peut-être du mal à trouver la «bonne personne», celle qui répond vraiment à leurs besoins. Un bon moyen de soigner le syndrome 
de «Où dois-je aller demander?», c'est de regrouper tous les bureaux en un seul point de services, et de bien former le personnel afin que tous vos agents puissent prendre en charge $95 \%$ des besoins du public.

Si vous estimez, après mûre réflexion, que le regroupement de vos bureaux d'accueil risque de dégrader le service rendu, faites des efforts en termes de formation transversale de vos agents. Si vos utilisateurs peuvent accomplir $95 \%$ de leurs démarches à n'importe quel bureau d'accueil, ils en seront certainement ravis.

\section{Éliminez}

Il serait un peu lâche et réducteur de passer sous silence une solution encore plus radicale: supprimer tous vos bureaux d'accueil. En fonction du contexte (budgétaire, administratif, $\mathrm{RH}$, appétit pour le changement, public à desservir), il est possible que vous travailliez dans une bibliothèque qui refusera même de l'envisager, et cela ne pose aucun problème (pour l'instant, en tout cas). Si vous travaillez dans une institution novatrice et pleine d'allant, dotée d'une administration et d'agents qui acceptent l'idée d'un changement radical dans la manière d'accueillir le public, c'est peut-être le bon moment pour vous séparer de tous vos bureaux d'accueil en même temps.

L'accueil et le renseignement peuvent aisément être assurés par des agents mobiles (tant qu'ils sont facilement identifiables par le public), même si vous ne possédez pas d'automates de prêt dans votre bibliothèque, car le personnel peut être équipé de douchettes portables sans fil pour enregistrer les emprunts n'importe où dans vos espaces. Cette possibilité mérite d'être explorée: pour guérir le syndrome de «Où dois-je aller demander?», le meilleur remède est peut-être de ne plus avoir du tout de bureaux. 


\subsection{Les gens reçoivent le type d'aide dont ils ont besoin}

\section{Difficulté $* * *$}

Vous est-il déjà arrivé de chercher désespérément le numéro de téléphone du service client d'une entreprise sur son site internet? Vous parcourez les pages «contacts » et «à propos» du site et votre incrédulité croît à chaque clic, lorsque vous découvrez que l'entreprise a décidé de supprimer son accueil téléphonique ou a fait de son mieux pour bien le cacher, afin que les clients utilisent les modes de communication qu'elle a choisi de privilégier, comme les tutoriels en ligne ou le service de tchat. Si cette expérience ne vous est pas étrangère, alors vous connaissez bien le sentiment de frustration ressenti lorsque vous n'êtes pas en mesure de choisir le moyen de communication qui vous convient pour entrer en contact avec une entreprise.

\section{Pourquoi c'est important}

Le scénario ci-dessus décrit la situation des services clients dans bien des entreprises de téléphonie mobile et autres fournisseurs d'accès internet. En analysant ce cas précis, nul besoin d'une batterie d'examens critiques pour se rendre compte que ces entreprises font clairement passer leurs propres intérêts (réduire les coûts, faire plus de profits) avant ceux de leurs clients (contacter le service client par le moyen de communication de leur choix).

Lorsque les bibliothèques prennent des décisions qui servent plus les intérêts de l'institution que ceux de ses utilisateurs, ces choix semblent être perçus moins négativement, notamment parce que les bibliothèques ne sont pas motivées par le profit. Cela dit, si ces décisions appauvrissent l'expérience des utilisateurs d'un service public, ils restent de mauvais choix. 
Voici un exemple un peu anecdotique, mais très répandu, de service rendu «à contrecœur » : les agrafeuses ou les paires de ciseaux en libre-service enchaînées aux meubles. Vous avez certainement déjà vu cela, peut-être même dans votre bibliothèque. Pourquoi attacher l'agrafeuse? Certes, si vous ne le faites pas, quelqu'un risque de l'emporter. Un bibliothécaire reconnaîtra peut-être que la chaîne représente un léger inconvénient, mais la verra cependant comme une nécessité. Et pour l'utilisateur? La présence de la chaîne est gênante, voire désobligeante. Le prix de remplacement d'une agrafeuse justifie-t-il cela? Sûrement pas.

On pourrait aussi évoquer la question des horaires «spéciaux» de certains guichets de service, comme ceux de prêt entre bibliothèques, de retrait des réservations, d'inscription, du département jeunesse, voire de prêt/ retour, comme dans bien des bibliothèques universitaires (BU) ouvertes en soirée, qui sont le plus sûr moyen d'empêcher un utilisateur d'avoir facilement accès au type de service dont il a besoin.

Le dernier diagnostic portait sur l'importance de fournir une aide aux gens où et quand ils en ont besoin. Le présent diagnostic complète l'équation en soulignant l'importance de fournir le type d'aide dont ils ont besoin.

Cela reviendra à leur proposer de l'aide selon toutes les modalités qu’ils désirent (sur place, en ligne, par téléphone), aux horaires les plus larges possibles, ou alors à couvrir tout le spectre allant du self-service à l'accompagnement pas à pas et à leur proposer, selon leurs besoins, une aide personnalisée sur rendez-vous ou en petits groupes.

\section{À vous de jouer}

La particularité de cet autodiagnostic est qu'il n'existe pas de mesure de base, et que le succès de votre bibliothèque dépend 
totalement des besoins de votre public et de votre capacité à y répondre. Chaque institution étant différente, il est recommandé d'utiliser une méthode d'observation des comportements. Vous pourrez ainsi évaluer si vous avez répondu où, quand et comme il le fallait aux besoins de vos utilisateurs. Ainsi que vous l'avez fait lors du précédent diagnostic, réalisez une petite enquête à la sortie de la bibliothèque, comprenant les questions suivantes:

1/ Avez-vous eu besoin d'aide pour trouver ce que vous étiez venu chercher aujourd'hui? (Oui / Non)

2/ Si oui, avez-vous pu obtenir le type d'aide que vous vouliez? (Oui / Non)

Vous marquez 25 points si vos membres répondent plus oui que non à la deuxième question; 12 points si les réponses se partagent équitablement et aucun point si vos membres ont majoritairement répondu non.

\section{Passez à l'action et proposez une aide mieux adaptée}

Si le CUTI de votre bibliothèque est bas à l'issue de cet autodiagnostic, vous avez du travail à faire pour réussir à répondre aux objectifs de vos utilisateurs et leur proposer le type d'assistance qu'il leur faut. Voici quelques idées qui vous aideront à en apprendre plus sur votre communauté et vous permettront de développer des services centrés sur les besoins des personnes.

\section{Du self-service à l'accompagnement pas à pas}

Le niveau de service parfait qui conviendrait à tous n'existe pas. Certains aiment comprendre les choses et résoudre les problèmes seuls; d'autres aiment regarder une courte vidéo avant de se débrouiller par eux-mêmes; d'autres enfin travaillent mieux avec un expert pour les épauler à chaque étape de leur recherche. Vous avez probablement des utilisateurs dans chacune de ces 
catégories, d'où la nécessité de bien définir la gamme de services que propose votre bibliothèque et de vous assurer qu'elle convient au plus grand nombre. Si vous réfléchissez aux options qui s'offrent à vos usagers en matière d'aide à la recherche bibliographique, pensez à proposer des guides en ligne, des tutoriels ou vidéos en libre-service, des bases de connaissances et une médiation proposée par les bibliothécaires.

\section{Couvrez toutes les modalités}

Vous souvenez-vous de l'histoire du service client de l'entreprise de téléphonie évoquée au début de ce chapitre? Ne faites pas la même erreur! Offrez autant de modalités d'accès à vos services que vous le pouvez: sur place, par mail, par téléphone, voire par SMS et en tchat.

\section{Un service flexible et personnalisé}

Le modèle d'aide tel qu'il est proposé à l'accueil (un par un, chacun son tour) ne s'adapte pas forcément à toutes les situations. Dans les bibliothèques universitaires, par exemple, les étudiants pourraient bénéficier de la possibilité de prendre rendez-vous avec un bibliothécaire au moment qui leur convient le mieux. $\mathrm{Ou}$ encore, un groupe d'étudiants travaillant sur le même projet pourrait vouloir être accompagné dans ses recherches par un bibliothécaire. Dans ces deux situations, le bureau de renseignement type n'est pas adapté pour fournir l'aide dont ces étudiants ont besoin. Vous serez donc bien inspiré d'introduire de la flexibilité dans la prise de rendez-vous et les séances de travail en groupes pour proposer des créneaux à des moments et dans des lieux qui conviennent à tous.

\section{Facilitez les connexions}

Si vous en avez les moyens, vous pouvez envisager un mécanisme qui permettrait à vos utilisateurs de s'entraider. Nombre 
d'entre eux sont probablement experts dans des domaines variés. Pourquoi ne pas les aider à se rencontrer? Vous pouvez voir ce qu'il est possible de faire sur les réseaux sociaux, travailler avec les associations étudiantes, accueillir des séances de tutorat assurées par des associations d'aide aux devoirs ou des dispositifs de lutte contre l'échec des étudiants.

Vous avez peut-être vous-même déjà observé l'entraide spontanée qui naît entre usagers lorsque tous les copieurs / imprimantes sont installés dans le même local et que ces systèmes, complexes, sont lancés depuis quelques années.

\section{Plongez en eaux profondes}

Comme vous avez déjà pu le remarquer, la réussite de votre bibliothèque est directement liée à la façon dont elle répond, ou non, aux besoins des membres de sa communauté. Pour en avoir une idée plus précise et aller de l'avant, vous devriez effectuer une plongée en eaux profondes et interroger vos utilisateurs. Voici quelques éléments que vous pourriez avoir envie d'expérimenter:

$\odot$ travaillez sur des scénarios avec des utilisateurs, une technique empruntée au design participatif. Vous pouvez élaborer, avec quelques personnes, des scénarios où ils ont besoin de l'aide de la bibliothèque. Une fois les scénarios créés, les associer à une phase de génération d’idées permettra d'imaginer des solutions pour que la bibliothèque puisse répondre à leurs besoins;

$\odot$ interviewez des utilisateurs: choisissez quelques personnes (cinq à sept devraient suffire) et questionnez-les sur les situations quotidiennes dans lesquelles elles cherchent une aide quelconque. Laissez la bibliothèque de côté, parlez de situations générales et demandez-leur comment et quand elles le font. Cela vous permettra de mieux comprendre la manière dont elles apprécient d'être aidées, et 
vous rendra plus perspicaces au moment d'élaborer votre offre de services;

$\odot$ développez des personas: jetez un œil au chapitre 2, paragraphe 2.2, pour savoir de quoi il s'agit, et pourquoi des personas pourraient être utiles à votre bibliothèque. Si vous arrivez à obtenir un groupe de 5 personas représentatives de vos utilisateurs, vous pourrez alors vous y référer pour développer des services adaptés à leurs besoins.

\subsection{Plus de lectures}

Gwynne S. C., "LUV and War at 30000 Feet", TexasMonthly, mars 2012. [En ligne] <www.texasmonthly.com/story/luv-andwar-30000-feet >.

Cet article détaille comment le service client et une culture organisationnelle positive ont été au cœur de la réussite de la compagnie aérienne Southwest Airlines.

Meyer D., Setting the Table: The Transforming Power of Hospitality in Business, New York, HarperCollins, 2006.

Un des livres favoris d'une bibliothèque dotée d'un excellent service client (la Darien Library). Ce livre contient les conseils avisés du célèbre restaurateur Danny Meyer.

Service Design Tools. [En ligne] < http://servicedesigntools.com>. Cet ensemble de ressources peut vous aider dans vos projets de conception de services en bibliothèque. 


\section{Chapitre 5. Règlements et services au public}

Le règlement, les procédures et les services aux publics sont intimement liés et révèlent comment une organisation se positionne vis-à-vis des publics qu'elle dessert. Si l'idéal type du «bon service» n'existe pas, toutes les bibliothèques peuvent s'efforcer de mettre en place certaines mesures pour servir au mieux leur public. Ce chapitre vous propose de faire le diagnostic du règlement, des procédures et des services aux publics dans votre établissement et d'envisager comment les faire évoluer.

\subsection{Votre bibliothèque a une philosophie des services}

\section{Difficulté **}

Voici un test rapide et amusant à faire: la prochaine fois que vous irez faire des courses, demandez au premier membre du personnel que vous croiserez quelles sont les valeurs du magasin: selon les lieux, elles sont souvent désignées sous le terme générique de philosophie des services. C'est une démarche distincte des chartes qualité, Marianne et autres bréviaires du service client. S’il s'agit d'une chaîne internationale, il y a toutes les chances pour qu'existe bien une philosophie des services explicite et présentée comme telle et une recherche rapide dans Google vous donnera la réponse. Pour autant, les personnes en contact direct avec la clientèle ne la connaissent pas toujours. Répétez l'exercice dans d'autres magasins, dans des chaînes d'hôtellerie, lors de vos appels à des SAV... Et gardez trace de vos résultats. Concluez cette petite expérience de terrain en posant la même 
question au collègue posté à l'un des bureaux d'accueil de votre bibliothèque. S'il vous répond d'une traite, sans erreur et avec le sourire, bravo! Inutile de faire ce diagnostic ni même le suivant; reprenez votre lecture un peu plus loin.

Si vous êtes encore en train de lire, c'est que vous voulez en savoir plus sur la formalisation de valeurs communes, que, fidèle au texte d'origine qui parle de service philosophy, l'adaptation française désignera sous le terme générique de philosophie des services, pour bien distinguer la démarche de celle des chartes qualité si prisées en France. Il y a en effet beaucoup à dire sur pourquoi et comment expliciter les valeurs qui doivent guider la mise en place des services dans votre bibliothèque.

\section{Pourquoi c'est important}

Lorsqu'on leur demande pourquoi elles font ce qu'elles font sur leur lieu de travail, beaucoup de personnes répondent simplement: «parce que c'est mon boulot». En pratique, la plupart des gens font ce qu'ils font parce que c'est leur travail et qu'ils en ont besoin pour joindre les deux bouts. Cela dit, lorsqu'il s'agit de travailler en contact avec le public, le service s'améliore lorsqu'il est guidé par des principes que tout le monde connaît, auxquels tout le monde croit et que chacun met en œuvre. Une manière de penser ces principes est de les considérer comme une philosophie des services.

Votre bibliothèque dispose-t-elle d'une philosophie des services? Si oui, alors 5 points pour vous. Ajoutez encore 5 points si vous l'appelez réellement Philosophie des services.

\section{Passez à l'action et créez votre philosophie des services}

Pour le dire simplement, une philosophie des services est un texte court d'une ou plusieurs phrases qui expliquent comment se positionne l'organisation face à la notion de service. Selon l'établissement, elle peut aussi s'appeler une philosophie 
d'entreprise, une charte du service clientèle ou toute autre variation sur ce thème. Une philosophie de service est un document qui a, d'une part, pour but de guider les personnels dans leurs interactions avec les usagers et, d'autre part, d'informer les usagers sur ce qu'ils peuvent attendre de l'organisation en termes de qualité de service. Si le terme philosophie des services vous paraît inadapté et un peu pompeux pour votre établissement, trouvez une formule mieux adaptée qui respecte à la fois votre intention d'expliciter des valeurs communes et la culture existante de votre bibliothèque.

Une recherche rapide dans Google des mots-clés «service philosophy" vous donnera de nombreux exemples de philosophies de services aux États-Unis. Voici trois exemples proposés par Amanda Etches et Aaron Schmidt, allant du plus lyrique au plus sobre.

\section{Valeur de base de Zappos Family: rendre un service wow (Whaou)!}

«Wow» est un mot court, simple et qui recouvre plein de choses. Pour qu'on dise que vous êtes «wow», vous devez vous démarquer, ce qui veut dire faire quelque chose d'un peu moins conventionnel et d'un peu plus innovant. Vous devez faire des choses qui vont au-delà des attentes des gens. Tout ce que vous faites doit avoir un impact émotionnel positif sur celui qui le reçoit. Nous ne sommes pas une compagnie moyenne, notre service n'est pas moyen, et nous ne voulons pas que les gens qui y travaillent se contentent d'être moyens. Nous attendons de chaque employé qu'il crée de l'émerveillement ${ }^{1}$.

1. [En ligne] <http://about.zappos.com/our-unique-culture/zappos-core-values/deliverwow-through-service $>$. 


\section{Le credo du Ritz Carlton}

Un hôtel Ritz Carlton est un lieu où notre mission est de procurer attention et confort authentique à nos hôtes. Nous promettons de fournir un service personnalisé et des équipements d'exception, qui permettent à nos hôtes de profiter d'une ambiance chaleureuse, détendue et raffinée. Une expérience Ritz Carlton aiguise les sens, donne une impression de bien-être et exauce jusqu'aux souhaits et besoins que les hôtes n'expriment pas².

\section{Bibliothèques et galeries de Cambridge (Canada)}

Un service personnalisé: rendre service aux gens en faisant attention à eux. Nos usagers sont tous des individus, et nous voulons les traiter comme tels. Nous nous efforçons de rendre un service personnalisé et individualisé avec courtoisie, chaleur humaine et sagesse $^{3}$.

\section{Mitonnez collectivement une philosophie des services} pour votre bibliothèque

Une bonne philosophie des services doit trouver un écho chez le personnel comme chez les usagers. Voici quelques éléments à garder à l'esprit lorsque vous l'élaborez.

\section{Soyez bref}

Personne n'a envie de lire des pages et des pages de bonnes intentions. Votre philosophie des services aura beaucoup plus d’impact si elle est courte et énergique.

\footnotetext{
2. [En ligne] <http://www.ritzcarlton.com/en/Corporate/GoldStandards/Default. htm\# credo >.

3. [En ligne] <http://www.ritzcarlton.com/en/Corporate/GoldStandards/Default. htm\# credo >.
} 


\section{Soyez centré sur l'usager, pas sur la bibliothèque}

Vous pouvez écrire toutes sortes de procédures qui expliquent au personnel comment se comporter au service du public, mais ce n'est pas le rôle de votre philosophie des services. Concentrezvous plutôt sur une formulation que vos usagers comprendront, apprécieront et qui exprimera clairement ce qu'ils sont en droit d'attendre de leur bibliothèque.

Impliquez les personnels dans le processus d'élaboration. Les bibliothèques sont pleines de livres consacrés à la création d'une culture d'entreprise et aux moyens d'obtenir, dès le départ, l'adhésion des personnels à ses valeurs.

1. Créez une petite équipe regroupant des membres du personnel de toutes catégories. Privilégiez les personnes en contact régulier avec le public et allégez au maximum la présence des cadres (un seul suffit).

2. Commencez par creuser collectivement ce qui existe déjà -inutile de réinventer la roue. Cherchez des philosophies de services dans tous les secteurs, public comme privé. Demandez à chaque membre du groupe d'en présenter trois, en expliquant pourquoi il les a choisies.

3. Toujours en groupe de travail, gardez-en trois. Décomposez chaque proposition et gardez ce qui trouve un écho chez chacun des participants.

4. Créez votre propre philosophie des services en utilisant tous les principes que vous avez appréciés chez les autres.

Tout au long du processus, communiquez souvent avec le reste du personnel en le tenant au courant des activités du groupe et en partageant les résultats à chaque étape. À l'issue de la quatrième étape, partagez la version de travail avec l'ensemble du personnel pour avoir leur avis. Reprenez et finalisez votre philosophie des services en tenant compte de ces remarques. 


\subsection{L'équipe connaît et fait vivre la philosophie des services}

\section{Difficulté **}

Félicitations! Votre bibliothèque a maintenant une philosophie des services. C'est un premier pas. Si vous y êtes parvenus en suivant la méthode proposée dans le point précédent, vous avez probablement déjà obtenu l'adhésion de l'équipe, ce qui est un élément décisif.

\section{Pourquoi c'est important}

Vous avez déjà compris pourquoi il est crucial d'avoir une philosophie des services. Aussi importante soit-elle, se contenter de la rédiger ne suffit pas: il est bien plus important de la faire vivre. En fait, rendre publique votre philosophie des services et ne pas la faire vivre au quotidien est pire que de ne pas en avoir du tout. Des promesses non tenues joueront en votre défaveur aux yeux de votre public, car elles révèlent un manque de fiabilité, minent votre crédibilité et, surtout, peuvent avoir un impact de longue durée sur la réputation de la bibliothèque, à l'opposé exact de ce que souhaite tout établissement. C'est pourquoi ce diagnostic est plus important encore que le précédent.

\section{À vous de jouer}

Tester votre bibliothèque sur ce point impose une double approche. Premièrement, interrogez jusqu'à dix personnes de la bibliothèque qui travaillent à l'accueil. Pour chaque personne pouvant citer la philosophie des services, marquez 1 point CUTI (10 points maximum sur cet item).

Une fois que vous aurez déterminé comment les gens s'en sortent lorsqu'il s'agit d'en rappeler la trame, vous allez vouloir tester 
s'ils la font vraiment vivre. L'approche est délicate, mais n'est pas impossible.

Vous avez probablement entendu parler du client mystère, technique souvent employée par les compagnies privées pour contrôler leurs salariés, afin de tester leurs connaissances sur les produits, les techniques de vente, leur investissement dans le service client, etc. Sans encourager les bibliothèques à espionner leur personnel, il est pertinent de tester si ce que font les employés répond aux attentes. Employer un client mystère est un moyen efficace de déterminer l'engagement de l'équipe dans la philosophie de service.

Il y a bien des manières d'utiliser cette technique pour savoir comment les personnels restituent la philosophie de service dans leur relation aux utilisateurs. Vous pouvez le faire de façon informelle, en interrogeant simplement famille et amis sur leurs dernières interactions avec le personnel de la bibliothèque, ou en demandant à un proche de s'adresser à un bureau d'accueil en particulier et de vous dire comment il a été reçu.

Il est également possible de recruter un vrai client mystère, pour entreprendre un certain nombre de démarches déterminées à l'avance le mettant en contact avec les points d'accueil, et noter combien d'interactions lui ont semblé conformes à la philosophie de service de la bibliothèque. C'est vous qui connaissez bien votre bibliothèque et êtes le mieux placé pour choisir la méthode la plus efficace. Pour noter cet élément, réalisez au moins cinq échanges avec des clients mystère (amateurs ou professionnels) et marquez un point pour chaque interaction qui prouve que les membres du personnel ont réellement pris à cœur la philosophie des services et la font vivre chaque jour. Participer au dispositif interministériel «Charte Marianne » vous permet de voir certains de vos services évalués tous les ans par des utilisateurs mystère, mais l'orientation de cette évaluation tend davantage à mesurer le respect de la lettre de la charte que son esprit. 
L'expérience du client mystère est positive dans les cinq cas: 5 points. Ce CUTI peut être noté de 0 à 5 points en fonction du résultat de l'expérience.

Additionnez cette estimation à la précédente, notée sur 10 points, pour arriver à un maximum de 15 points à l'issue de ce diagnostic.

\section{Passez à l'action: quelques moyens d'encourager le personnel à connaître et faire vivre votre philosophie des services}

Il est assez facile d'être motivé quand quelque chose est tout beau, tout neuf. Dans le meilleur des cas, au moment où vous élaborerez et lancerez votre philosophie de service, le personnel sera très motivé. Le plus ardu sera alors de maintenir cette motivation. Voici quelques idées pour y arriver.

Répétez, répétez, répétez

Ne sous-estimez jamais le pouvoir de la répétition. Quand vous dites quelque chose de manière répétée, cela laisse une trace. Misez sur cette psychologie élémentaire et rappelez votre philosophie de service dès que l'occasion se présente. Imprimez des posters qui reprennent les formules choisies et affichez-les un peu partout dans votre bibliothèque, tout particulièrement à des endroits où le personnel peut les voir. Imprimez des versions en format carte postale (si vous suivez notre conseil et que vous faites court, cela devrait être facile!) à donner aux nouveaux personnels.

Si vous avez un livret d'accueil ou un guide d'accueil pour les nouveaux personnels, imprimez sur la première page votre philosophie de service. Assurez-vous toujours que, lorsque vous la rappelez au personnel, vous le faites dans un contexte pertinent, afin de ne pas la passer en boucle comme un disque rayé. Par exemple, 
chaque fois que vous prenez une décision ou changez quelque chose dans la bibliothèque, mettez en exergue une formule du genre "en accord avec notre philosophie de service sur X..., nous avons décidé de faire XXX...», lorsque vous informerez vos collègues. Remplacez le X par ce que vous voulez!

\section{Donnez en modèle les bonnes pratiques}

Les bonnes pratiques peuvent être contagieuses. Rien ne motive autant à faire vivre une philosophie des services que de voir les autres membres du personnel incarner cette philosophie et y conformer leur comportement. Si vous ne travaillez pas vousmême à l'accueil, traitez vos "clients» (qui peuvent être des membres du personnel) de la manière dont vous voudriez qu'ils traitent les utilisateurs de la bibliothèque.

\section{Levez le voile sur le client mystère}

Si vous avez l'intention de tester le personnel à propos de la philosophie des services, vous devriez le lui dire. Dire qu'il y aura des clients mystère (qu'il s'agisse d'un client mystère professionnel ou des approches informelles listées ci-dessus) est une marque de confiance envers le personnel et envoie aussi le message que vous prenez au sérieux l'implémentation de la philosophie des services.

\section{Soyez attentif lors des recrutements}

C'est un lieu commun de dire que les compétences (savoir-faire) sont plus faciles à acquérir que les attitudes (savoir-être). Les bibliothèques sont des organisations qui rendent des services: or, tout le monde n'est pas en mesure de se sentir performant, ni même à l'aise, à un poste d'accueil. Si vous le pouvez, infléchissez vos pratiques de recrutement pour engager des personnes dont les savoir-être sont compatibles avec la culture et la philosophie des services de votre bibliothèque. 
Tenez à jour et retravaillez régulièrement votre philosophie des services

Rien de mieux pour réactiver votre philosophie des services que des relectures et révisions de routine. Revoir votre philosophie des services tous les 18 à 24 mois devrait être une priorité dans votre établissement. Engagez tout le personnel dans le processus de révision: ce sera l'occasion de prendre le pouls de l'organisation (et du personnel) en vous demandant s'il s'agit toujours de quelque chose en quoi vous croyez et qui suscite toujours l'adhésion générale. Faites évoluer les formulations le cas échéant.

\subsection{II y a aussi peu de procédures et de règles que possible}

\section{Difficulté $* *$}

Au premier abord, l'idée d'avoir peu de règles et procédures peut sembler contre-intuitive. Les procédures sont à la base de bien des services, et vous pouvez donc vous attendre à avoir des règlements relatifs à toutes les interactions ou scénarios possibles, afin de rendre les choses plus claires pour les équipes et de gérer les demandes des utilisateurs de manière appropriée. On peut pourtant envisager les choses d'une autre manière.

\section{Pourquoi c'est important}

Beaucoup de règles et procédures sont des restrictions superflues. Trop de règles limitent les membres du personnel de manière négative: elles restreignent leur capacité à exercer leur discernement sur la manière d'interagir, de répondre ou de rendre service aux gens.

Un cas souvent cité en exemple est le règlement de Nordstrom, une enseigne de vente en ligne américaine. Nordstrom est bien connue pour n'avoir qu'une seule règle en matière d'interaction 
avec les clients qui est tout simplement «Faire preuve de bon sens en toutes circonstances». Le manuel du personnel se résume à une carte postale qui cite la «règle unique » et met un point d'honneur à répéter qu'il n’y a pas d'autre règle (voir < http://about. nordstrom.com/careers and the Nordstrom way to Customer service excellence >, de Robert Spector et Patrick McCarthy).

Comment limiter le nombre de procédures peut-il améliorer l'expérience vécue par les utilisateurs dans votre bibliothèque? Pour commencer, cela minimise les risques de confusion chez le personnel, pour une bonne et simple raison: moins il y a de règles, plus il est facile de s'en souvenir. Si votre organisation est dotée d'une procédure pour chaque situation, votre équipe en contact avec le public sera contrainte de consulter un gros manuel au cours de chaque interaction avec un utilisateur: c'est aussi encombrant qu'inutile. En fait, avoir moins de règles signifie donner plus de latitude aux personnels pour exercer leur discernement et faire vraiment passer l'usager au premier rang de leurs préoccupations.

\section{À vous de jouer}

Il serait tentant (et facile) de se contenter de vous demander de compter toutes vos procédures liées aux services en cours et d'enlever un point pour chacune.

Plutôt qu'un tel recensement, voici un exercice un peu plus réflexif pour évaluer l'état des règlements dans votre bibliothèque. Essayez de dresser une liste des «Non» à chaque bureau d'accueil de votre bibliothèque. Demandez aux agents de chaque bureau de noter toute question ou requête d'un utilisateur qui reçoit une réponse négative. Tenez cette liste des «Non» pendant au moins un mois: à la fin de ce mois d'observation collective, faites le bilan de toutes les situations de refus et mettez en face de chaque «Non» la procédure ou pratique en place qui a 
justifié ce «Non». Commencez avec 15 points, et enlevez un point par procédure différente sur cette liste. Reportez le CUTI obtenu dans le tableau de synthèse.

Si vous avez plus de quinze procédures différentes sur la liste des «Non», 0 point pour votre bibliothèque.

\section{Passez à l'action : regardez de près vos procédures}

Si votre CUTI est faible après cet autodiagnostic, votre bibliothèque souffre peut-être d'hypertrophie réglementaire. Voici quelques pistes pour la soigner:

$\odot$ revoyez toutes vos procédures et règlements;

$\odot$ reprenez vos règlements un à un, demandez-vous pourquoi ils ont été mis en place et si vous en avez encore vraiment besoin. Le nombre de règles qui restent en place pour la seule raison que "nous avons toujours fait comme ça» est effrayant;

$\odot$ demandez de l'aide aux personnels qui se trouvent au contact du public.

Les personnels d'accueil sont probablement ceux qui ont affaire le plus régulièrement à vos règlements et qui les connaissent le mieux. Impliquez-les en leur demandant de choisir les trois règlements les plus inutiles, gênants ou difficiles à faire respecter dans leur travail. Débarrassez-vous des règlements qui ne servent à rien, retravaillez ceux qui suscitent de la gêne ou sont compliqués à faire respecter.

Revoyez régulièrement cette liste des «Non». Refaites l'exercice de la liste des «Non" de manière régulière, environ tous les deux ou trois ans. Cet exercice peut vraiment vous aider à faire basculer votre organisation d'une culture du «Non » à une culture du «Pourquoi? » et du «Pour quoi?». 


\subsection{Les règlements de la bibliothèque donnent des marges d'action et de décision aux professionnels}

\section{Difficulté $* * *$}

On a déjà dit que trop de procédures diminuent la qualité de l'expérience vécue par l'utilisateur et, de surcroît, entravent et noient les personnels. La question de la qualité des procédures est tout aussi importante que celle de leur quantité. Même si votre bibliothèque ne souffre pas d'hypertrophie réglementaire et que vous avez un score de 15 points au dernier diagnostic, cette question-ci est tout aussi importante pour offrir une expérience de qualité aux membres de votre communauté.

\section{Pourquoi c'est important}

Quantité de règles, de recommandations, d'instructions et de procédures sont parfois nécessaires pour faire le travail demandé. Si vous travaillez dans une start-up et que la seule procédure en vigueur est: "Jette le manuel et fais de ton mieux", vous vous sentirez peut-être délivré d'un poids. Mais si votre boulot est de monter des meubles en kit, vous serez vraiment démuni devant une telle directive. Pourquoi? Parce que monter un meuble en kit exige de suivre un mode d'emploi!

Heureusement pour les bibliothécaires, monter des meubles - du moins ceux des utilisateurs (mais ce serait une possibilité originale de nouveau service?) - ne fait pas partie de leurs missions.

Cela ouvre la possibilité de réduire le nombre de règlements, de n'en garder ou réécrire que quelques-uns, concis, qui laissent des marges d'action aux agents pour prendre des décisions à la volée, sans se référer à un lourd appareil réglementaire. 
Souvenez-vous des vertus de la «Règle unique» de Nordstrom: «Utilisez votre bon sens dans toutes les situations». À l'évidence, l'idée sous-jacente est bien de donner des marges d'action pour que vos employés puissent utiliser leur bon sens. Pour cela, il vous faut avoir confiance dans leur capacité à prendre des décisions. Une fois cela acquis, vous leur donnez le pouvoir d'utiliser leur discernement. Il s'agit d'un cercle vertueux qui s'auto-entretient et dont tout le monde sort gagnant.

\section{À vous de jouer}

Ce diagnostic est plus difficile que d'autres à dresser. Vous voulez déterminer si les procédures en place donnent des marges d'action et de décision à vos personnels ou si, au contraire, elles entravent leurs possibilités de rendre un service exceptionnel. Pour évaluer comment se situe votre bibliothèque, adoptez une approche en deux volets.

Tout d'abord, interrogez une dizaine d'agents d'accueil pris au hasard pour déterminer comment ils se sentent lorsqu'ils doivent faire appliquer un règlement de la bibliothèque. Se sententils limités dans leur capacité à rendre un service de qualité? $\mathrm{Au}$ contraire, se sentent-ils autorisés et en mesure de le faire?

Marquez un point CUTI à chaque fois qu'un membre de votre équipe dit qu'appliquer une procédure de la bibliothèque lui donne l'impression d'avoir une marge d'action et de décision positive. La note maximale est de 10 points.

Le deuxième volet met en œuvre une méthode d'analyse des opinions (cf. chapitre 2), permettant de savoir comment les usagers ressentent le type de service qu'ils ont reçu.

Elle peut prendre la forme d'un sondage très rapide sur dix usagers pris au hasard: au moment où ils quittent un espace d'accueil de la bibliothèque, demandez-leur s'ils ont l'impression d'avoir 
reçu un service adapté à leurs besoins et à leur situation particulière. Il est sans doute judicieux de leur demander au préalable le but de leur passage, dans la mesure où la question concernant le service reçu n'est pas forcément adaptée à tous les cas. Marquez un point CUTI à chaque fois qu'un utilisateur dit que le service qu'il ou elle a reçu était tout à fait adapté à son besoin particulier. La note maximale est de 10 points.

Additionnez les deux CUTI ci-dessus, et reportez le total dans le tableau de synthèse, sur 20 points.

\section{Passez à l'action}

\section{Les règlements peuvent laisser plus de liberté au personnel}

Si votre bibliothèque a eu un mauvais score, gardez bon espoir. Utiliser les règlements comme un moyen de donner plus de latitude d'action au personnel plutôt que de prescrire des comportements précis constitue une rupture par rapport aux habitudes installées. Il y a certainement un gros travail à faire sur ce point. Voici quelques stratégies utiles.

Tenez compte des résultats des derniers points de diagnostic. Au risque de paraître rabâcher, il faut revenir sur des points capitaux:

$\odot$ avoir une philosophie de service;

$\odot$ la faire vivre;

$\odot$ réduire le nombre de règlements et de procédures dans votre bibliothèque.

Ces trois points vous mettront sur la bonne voie et permettront à votre personnel de fournir le meilleur service possible.

\section{Changez de culture}

Ce n'est pas une suggestion faite à la légère. Nous savons que vous n'allez pas changer la culture de votre organisation en une 
nuit. Ce type de changement, touchant aux représentations professionnelles, est difficile. C'est pourquoi cela va vous demander beaucoup de travail.

Vous aurez besoin de l'accord des cadres, ainsi que de celui de votre tutelle, du président de l'université, si vous êtes une bibliothèque universitaire, ou des directions de la culture, si vous êtes dans une bibliothèque territoriale. Dans ce contexte, l'enjeu d'un changement de culture porte sur deux points:

$\odot$ faire du service (et partant de là, de l'expérience de l'utilisateur) votre priorité numéro un;

$\odot$ infléchir votre politique $\mathrm{RH}$, votre gestion prévisionnelle des emplois et compétences, votre organigramme et vos recrutements en fonction de cet objectif.

\section{Recrutez les bonnes personnes}

Agir sur le recrutement: voilà une idée qui ne va pas prendre corps en une nuit. Il y a de bonnes raisons de penser que lorsque vous recrutez quelqu'un, il est préférable de rechercher des savoir-être plutôt que des compétences ou des savoir-faire trop spécifiques. Une compétence peut toujours s'acquérir, moyennant une formation pratique ou un tutorat attentif. Un savoirêtre touche à une dimension plus personnelle des individus, au croisement de la culture et de la personnalité, et contribuer à le développer dans une certaine direction ne peut être décidé unilatéralement par un $\mathrm{N}+1$.

Autant ne pas délibérément vous compliquer la tâche lorsque vous recrutez des personnels d'accueil: apprenez à être attentif et à rechercher des indices, au moment des entretiens de recrutement, sur les dimensions humaines et relationnelles, sans vous focaliser sur d'éventuelles lacunes purement techniques.

En France, les concours mesurent surtout, dans la phase d'admissibilité, des connaissances orientées métiers et des savoir-faire en 
matière d'aisance rédactionnelle: cela ne simplifie pas les choses. Cela dit, rien ne vous empêche de recevoir, avant un mouvement ou une mutation, toutes les personnes qui souhaitent venir travailler dans votre bibliothèque et de classer les candidatures d'après leur savoir-être et leurs expériences réussies de médiation plutôt que d'après la maîtrise d'un module particulier de prêt/retour, le rang de sortie de diplôme ou l'épaisseur de leur CV. Si l'occasion vous est donnée de participer à des jurys de concours, réfléchissez à la façon de valoriser et révéler les savoir-être des candidats plutôt que leurs seules connaissances (cf. les premières pages de < http://www.fonction-publique.gouv.fr/files/files/publications/coll_ressources_humaines/guide_jury.pdf >).

\section{Le dernier règlement}

Il y a quelque chose de paradoxal à recommander de faire un règlement pour donner de la marge de manœuvre au personnel.

Une des histoires préférées d'Aaron Schmidt et d'Amanda Etches à propos de l'autonomie d'action des employés vient de la chaîne d'hôtels de luxe Ritz Carlton. Le Ritz Carlton a élaboré une stratégie complète pour aider son personnel à faire vivre ce «credo», incluant un «refrain», les «trois étapes du service» et le « $6^{e}$ diamant», une formule sur le service exemplaire (voir < http://www. ritzcarlton.com/en/about/gold-standards $>$ ). De plus, chaque membre du personnel peut, sans en référer à quiconque, aller jusqu'à une dépense de 2000 dollars pour satisfaire un hôte. Dans un article de Forbes daté de 2009, Simon F.Cooper, alors président du Ritz Carlton, décrivait ainsi le niveau d'investissement dans la qualité du service:

Quand vous dites: «Peut aller jusqu'à 2000\$», vous trouvez toujours quelqu'un pour dire: «c'est super, il ne s'agit pas juste d'offrir un film lorsque la chambre n'est pas prête, c'est vraiment une belle somme.» Elle n'est pas souvent utilisée, mais c'est une preuve de 
la confiance que nous avons dans la capacité de jugement de notre personnel... L'idée, c'est de faire quelque chose, de créer un séjour vraiment merveilleux pour chacun de nos hôtes. Il ne s'agit pas seulement de répondre à un problème. Un exemple: si quelqu'un découvre que c'est l'anniversaire d'un hôte, il fait envoyer du champagne et un gâteau dans sa chambre. D'après ce que je vois depuis mon bureau, la plupart du temps, il n’a pas été question de venir à bout d'un problème, mais d'utiliser les 2000 \$ pour créer une expérience hors du commun.

Cette procédure du Ritz Carlton donne de manière effective du pouvoir aux employés, en faisant preuve d'un niveau de confiance élevé dans leur capacité à prendre de bonnes décisions au bénéfice de leurs clients.

N'en concluez pas qu'il faille donner toute latitude au personnel de la bibliothèque de dépenser des milliers d'euros pour chaque usager. Réfléchissez malgré tout aux outils que l'on peut mettre entre les mains des agents pour leur donner le pouvoir de rendre l'expérience vécue par les utilisateurs vraiment exceptionnelle. Les pistes sont nombreuses:

$\odot$ ouverture d'espaces ordinairement non accessibles au public;

$\odot$ prêt de matériel inhabituel (de l'agrafeuse au vélo, en passant par les chargeurs et le petit matériel audiovisuel);

$\odot$ distribution de friandises ou d'objets promotionnels lors de la promotion de certains services;

$\odot$ voire, mais cela ne s'improvise pas, invitation à des événements particuliers (fête de rentrée ou de fin d'année, accueil des Erasmus autour d'un gâteau de bienvenue...).

Quoi que vous décidiez, résumez-le en une formule courte et bien tournée, puis assurez-vous que votre personnel la connaisse et la fasse vivre. Si votre organisation s'est engagée dans une 
démarche qualité et que vous avez recruté les bonnes personnes, cela ne devrait pas poser problème.

\subsection{L'équipe de la bibliothèque est accueillante et serviable}

\section{Difficulté $* * *$}

Il a déjà été répété à plusieurs reprises qu'un bon service public était l'une des bases d'une bonne expérience pour l'utilisateur. La qualité des interactions humaines peut nouer ou dénouer une situation problématique. Avez-vous déjà vécu un délicieux dîner dans un bon restaurant gâché par un serveur désagréable? Ou, en avion, un problème de vol rattrapé par une hôtesse ou un steward adorable qui a tout fait pour être sûr que vous étiez bien? Ces deux exemples montrent comment l'interaction avec une personne peut aussi bien gâcher une bonne expérience qu'en rattraper une mauvaise. Ce diagnostic porte entièrement sur l'importance majeure d'un bon service pour la qualité de l'expérience des usagers de votre bibliothèque.

\section{Pourquoi c'est important}

Vous avez probablement déjà entendu l'adage: "Une personne satisfaite en parle à une ou deux autres; une personne mécontente en parle à dix fois plus». C'est une raison suffisante pour se convaincre de l'importance d'être attentif à l'état du service public dans votre bibliothèque.

Cela impose de revenir sur un autre point déjà évoqué, qui n'est jamais aussi vrai qu'en matière de service à l'usager: un savoirfaire technique peut toujours s'acquérir, moyennant une formation pratique. Modifier un savoir-être ne peut être décidé unilatéralement par un $\mathrm{N}+1$, et on ne peut compter ni sur la formation continue ni sur l'imitation pour arriver à un service à l'utilisateur 
authentiquement bon. Recruter intelligemment, lorsque cela est possible, est la voie de progrès la plus rapide.

Amener tous les membres d'une équipe constituée à se conduire de manière sincèrement accueillante et serviable est l'un des points les plus difficiles de la démarche globale, pour deux raisons:

$\odot$ premièrement, si votre organisation n'a pas mis l'accent de longue date sur l'importance d'un bon service, vous aurez à composer avec des agents en première ligne qui ne sont pas forcément tous aptes à relever le défi;

$\odot$ deuxièmement, même si vous êtes en mesure de recruter de nouveaux personnels d'accueil, il n'est pas toujours facile d'identifier ou d'attirer des professionnels des bibliothèques qui ont déjà un goût affirmé pour les services.

\section{À vous de jouer}

Pour évaluer comment votre organisation s'en sort sur ce point, tournez-vous vers les meilleurs juges de l'expérience des services dans votre bibliothèque: ceux qui les utilisent.

Faites une enquête d'opinion pour demander à quinze de vos usagers s'ils sont satisfaits de l'accueil qu'ils ont reçu lors de leur dernière visite, et si les membres du personnel avec lesquels ils ont échangé leur ont semblé vraiment désireux de les aider (plusieurs sondages de ce type ont été suggérés dans ce chapitre: il est possible de les regrouper au sein d'une même enquête pour ne pas lasser vos usagers en les inondant de sondages). Il faut que les sondés puissent rendre de manière la plus anonyme possible leurs réponses - si vous distribuez en personne la quinzaine de questionnaires, demandez à ceux qui les remplissent de ne pas vous les rendre en mains propres, mais de les déposer à un autre endroit, par exemple, dans une boîte à l'entrée de la bibliothèque. Plusieurs études ont montré que les gens viennent 
rarement se plaindre à quelqu'un quand ils ont reçu un service qui n'est pas à la hauteur: vous pourriez tomber sur des usagers qui vous disent que tout va bien alors que leurs commentaires écrits diront toute autre chose. Marquez un point pour chaque usager qui a une expérience positive. Le score maximal est de 15 points.

\section{Passez à l’action et améliorez le service}

La plupart de ces idées ne peuvent pas être mises en œuvre du jour au lendemain. Vous avez peut-être remarqué que tout ce chapitre est noté trois étoiles. De tels changements exigent approbation administrative, discussions approfondies, négociation avec les représentants du personnel et, plus largement, consensus et approbation de l'ensemble du personnel. Décréter qu'on s'engage dans un service de qualité est aisé, faire vivre cet engagement prend du temps et demande de gros efforts. Le jeu en vaut la chandelle.

\section{Encouragez l'autonomie responsable des professionnels}

Autonomie responsable est une manière appauvrie de traduire le terme original empowerment: cette notion a été définie comme «le mécanisme par lequel les gens, les communautés, les organisations augmentent la maîtrise de leur vie ». Elle désigne la capacité d'agir, le processus par lequel une personne peut elle-même contrôler sa situation et améliorer ainsi ses conditions de vie, de travail ou son environnement.

Le dernier diagnostic était consacré à la façon de permettre à vos personnels de s'appuyer sur leur discernement et leurs compétences pour rendre agréable l'expérience de l'utilisateur. Si vous avez déjà le personnel qu'il vous faut en première ligne, mettre en œuvre ce genre de choses devrait aller tout seul et pourrait même se révéler amusant. Si ce n'est (pas encore!) le cas, vous 
aurez intérêt à ne pas commencer par là et à y revenir un peu plus tard.

\section{Repositionnez le personnel}

Si votre organisation commence tout juste à faire du service une priorité, vous devez composer avec certains personnels d'accueil qui manquent quelque peu d'assurance ou d'aisance lorsqu'on en vient aux compétences interpersonnelles.

Si vous êtes dans cette situation, vous pouvez envisager une réorganisation de transition pour accompagner ces personnels vers des fonctions internes, afin qu'ils soient moins en contact direct avec les usagers de la bibliothèque et, du même coup, que la bibliothèque utilise mieux leurs autres compétences. Si quelqu'un n'est ni cordial ni affable à l'accueil, il y a de bonnes chances qu'il ou elle ne se sente de toute manière pas à l'aise à ce poste. Lui proposer moins d'accueil pourrait bien au final améliorer ses conditions de travail, voire celles de ses collègues. En parallèle, recherchez activement au sein du personnel des gens qui sont à l'aise dans leur relation aux autres et ont été jusqu'ici cantonnés à du travail en interne: proposez-leur des missions d'accueil et de service.

Parvenir à cela est très complexe dans la plupart des structures où la continuité des plannings d'accueil repose sur des équilibres délicats et bien ancrés dans l'histoire professionnelle de l'institution et de ses acteurs. Ce n'est toutefois pas impossible.

\section{Recrutez les bonnes personnes}

C’est un point crucial, qui mérite d'être répété une nouvelle fois.

Si vous êtes en mesure (veinard que vous êtes!) de recruter du personnel d'accueil, assurez-vous de recruter des personnes qui non seulement sont accueillantes et serviables, mais qui ont aussi de l'empathie pour les autres. Cherchez celles qui ont eu une expérience réussie en la matière. 
On ne reviendra pas sur l'importance, en France, de faire évoluer les représentations sur les compétences clés des bibliothécaires et de mieux valoriser les savoir-être par rapport aux pures connaissances administratives, techniques et bibliothéconomiques, à toutes les étapes de la vie professionnelle: formation initiale, sélection en Master Pro, concours, recrutement au sein d'un établissement. Sans un mouvement d'ampleur en ce sens, passer d'une organisation fondée sur le traitement documentaire à une organisation orientée vers la qualité de l'expérience vécue par l'utilisateur restera difficile. Tous les espoirs restent permis, de très nombreuses personnes ouvertes et attentives aux autres ayant, même sans y être encouragées, infiltré les bibliothèques françaises.

\section{Encouragez la transparence}

Rien n'illustre mieux un engagement en faveur de la qualité du service que d'encourager les retours de la part de la communauté que l'on sert, et d'être transparent à ce sujet. Mettez en place une boîte à commentaires dans votre bibliothèque et en ligne, pour encourager les usagers à vous dire comment vous vous en sortez - et partagez ces avis avec tout le personnel de la bibliothèque. Pour un équivalent en ligne, essayez un réseau social comme Facebook ou Twitter, ou testez une plate-forme qui collecte les avis. Soyez réaliste et sincère lorsque vous répondez à ces commentaires: acceptez les compliments avec grâce et excusez-vous si besoin.

\section{Construire de l'empathie}

On a dit et répété qu'une bonne attitude ne pouvait être enseignée. Cependant, il est possible, si ce n'est d'apprendre l'empathie, du moins de la révéler et de l'affûter. Une excellente manière d'encourager les agents à s'occuper de l'expérience usager est de les aider à comprendre ce que ressent un usager lorsqu'il utilise 
les services de la bibliothèque. Et la meilleure manière de développer compréhension et empathie est de les encourager à se mettre à la place d'un utilisateur. Pour ce faire, instaurez une journée «Dans la peau d'un usager» pour l'équipe d'accueil, idée proposée pour la première fois par le bibliothécaire Brian Herzog, < http://www.swissarmylibrarian.net/2008/10/07/worklike-a-patron-day >. Ce jour-là, les agents devront essayer de faire leur travail quotidien en utilisant les mêmes ressources, les mêmes outils et les mêmes interfaces que vos usagers. Comme Herzog le recommande dans son billet de blog, le personnel doit:

$\odot$ entrer et sortir de la bibliothèque par l'entrée du public (et non par celle du personnel);

$\odot$ utiliser les toilettes publiques;

$\odot$ utiliser les ordinateurs en libre accès pour travailler;

$\odot$ réserver les salles de travail de la bibliothèque pour les réunions;

$\odot$ respecter l'ensemble des règlements et procédures de la bibliothèque.

Vous pouvez aussi envisager d'équiper le personnel avec le même mobilier que les usagers, et les encourager à utiliser systématiquement les interfaces publiques (plutôt que professionnelles) pour tous vos outils en ligne. Encouragez les personnels à noter leurs observations, à avoir une attitude réflexive sur cette expérience, et prenez un peu de temps le jour suivant pour en faire une restitution en petit groupe.

Un autre moyen de renforcer l'empathie est de faire une enquête contextuelle en y associant le personnel d'accueil. Comme cela a été rapidement dit au chapitre2, une enquête contextuelle est une méthode de recherche comportementale où les participants observent des usagers qui doivent mener à bien des tâches 
spécifiques dans la bibliothèque. Un exercice contextuel réussi qui associe les personnels exigera une formation préalable, pendant laquelle les agents seront invités à faire des hypothèses, à observer attentivement, à prendre des notes et à prendre du temps à la fin de l'exercice pour synthétiser les données qu'ils ont collectées et réfléchir à ce qu'ils ont observé. Une fois encore, travaillez en groupe à la restitution pour donner à tous l'occasion de discuter tant sur ce qu'ils ont observé que sur l'exercice en général.

Vous constaterez que les exercices du type «Dans la peau d'un usager » couplés à une enquête contextuelle renforceront l'empathie de vos agents et les transformeront en de meilleurs ambassadeurs des services au sein de votre bibliothèque.

\subsection{La qualité du service est homogène au sein de l'institution}

\section{Difficulté $* * *$}

Les bibliothèques sont avant tout un ensemble de services. Vous rendez un service lorsque vous organisez une heure du conte pour les moins de 5 ans; vous rendez un service lorsque vous aidez quelqu'un à imprimer son CV; vous rendez un service lorsque vous commandez un article par le biais du prêt interbibliothèques ou encore lorsque vous concevez une interface pour faire des recherches dans les ressources en ligne acquises par la bibliothèque.

La plupart du temps, chacun de ces services est rendu par plusieurs personnes, issues de plusieurs «services» ou «départements » et selon différentes modalités (certains en face-à-face, d'autres en ligne, d'autres encore par téléphone).

Il faut garder à l'esprit que, du strict point de vue des utilisateurs, il s'agit toujours de services. Peu leur importe à quel «service» ou «département» de la bibliothèque ils ont affaire: ces 
personnes recherchent simplement un service de qualité homogène lorsqu'elles ont affaire à la bibliothèque.

\section{Pourquoi c'est important}

Si vous avez prêté attention à Twitter ces temps-ci, vous avez probablement été témoin ou bénéficiaire d'un service hétérogène.

Alors que Twitter a commencé comme un petit coin d'Internet, où des amis pouvaient tenir de brèves conversations avec des membres de leur réseau, il est devenu maintenant une énorme plate-forme de "service clientèle ». Chaque entreprise de quelque importance y est présente et a engagé du personnel dédié pour répondre aux tweets sur la compagnie (réclamations ou compliments). Pour les clients qui sont sur Twitter, c'est formidable! Ils bénéficient là d'un meilleur service, plus rapide, et plus humain: ils sont donc ravis. Les gens qui n'ont pas de compte Twitter? Tant pis pour eux: qu'ils continuent à taper \#1, \#2 ou \#3 sur des standards téléphoniques à rallonge ou à attendre de longues minutes d'être mis en relation avec un SAV à peu près francophone délocalisé dans un centre d'appel.

Où veut-on en venir? À cette simple constatation: offrir de meilleurs services à certains usagers sous prétexte qu'ils ont opté pour la dernière plate-forme à la mode n'est pas satisfaisant.

De la même manière, il n'est pas bon de rendre un meilleur service à un usager qui se trouve à l'accueil de la bibliothèque plutôt qu'à celui qui aura choisi d'envoyer sa question par mail. Et pourtant, les bibliothèques font tout le temps ce genre de choses. Il y a des chartes de qualité pour l'accueil in situ, mais ce ne sont pas les mêmes pour les mails, les services de référence en ligne, les réseaux sociaux ou le téléphone.

Cet exemple est centré sur les types d'interactions: de la même manière, il est important de penser à l'homogénéité des services 
entre départements. Bien souvent, chacun est enfermé dans son propre silo et prend des décisions à l'échelle de son service sans penser à en faire part à ses collègues des autres départements, même si les bureaux ne sont séparés que de quelques mètres dans le même bâtiment. Alors que cela semble hallucinant lorsqu'on prend du recul, vous connaissez sans doute une bibliothèque où les prix de photocopie, les règles de prêt ou les pénalités diffèrent d'un «département» ou d'une "section» à l'autre, au sein d'un même établissement. Vous vous dites que ce genre de chose ne se produit jamais dans votre bibliothèque: il est temps de vous en assurer.

\section{À vous de jouer}

Évaluer l'homogénéité du service peut être un peu délicat, car on ne peut pas comparer des pommes, des poires et des scoubidous. Dressez donc d'abord une liste des services comparables rendus par plusieurs départements ou de différentes manières au sein d'un même service.

Prenez le renseignement bibliographique, par exemple. Vous pouvez fournir un service de renseignement bibliographique en personne, par tchat ou par téléphone ou dans plusieurs départements thématiques dotés chacun d'un bureau de renseignement. Si tel est le cas, le renseignement bibliographique est un candidat idéal pour une évaluation comparative. Comparez des éléments mesurables comme les horaires, les files d'attente et les délais de réponse selon les lieux ou les types d'interactions.

Si vous avez plus d'un département qui propose un service de photocopie et d'impression aux usagers, voilà un autre bon sujet d'évaluation croisée. Même chose pour les règles d'emprunt, de prolongation et les pénalités.

Vous pouvez aussi jeter un œil à la programmation des événements dans votre bibliothèque ou à la réservation des salles de 
travail. Plusieurs départements proposent des activités ou des salles réservables? Si tel est votre cas, cela peut valoir la peine de regarder de plus près comment chaque département gère les réservations et les listes d'attente, par exemple.

Pour évaluer l'homogénéité du service de votre bibliothèque, vous pouvez comparer trois types de services différents et leurs déclinaisons au sein de votre établissement.

Les trois services sont homogènes: 10 sur 10 !

Deux sur trois: attribuez-vous 7points.

Un sur trois: vous avez 3 points.

Si aucun des services comparés n'est homogène au sein de votre établissement, 0 point.

\section{Passez à l'action et homogénéisez les services entre départements et types d'interfaces}

Si votre bibliothèque a eu la note maximale sur ce point, bravo.

Tous ceux qui ont travaillé dans de grandes organisations savent combien il est difficile d'atteindre un tel niveau d'homogénéité de service. Il est tout à fait compréhensible, si votre score est moins bon, qu'incohérences et divergences se soient glissées dans toutes vos pratiques. Voici quelques idées pour vous aider à vous mettre sur la piste d'une meilleure cohérence entre services d'une même bibliothèque.

\section{Engagez-vous}

La cohérence des services fait partie des choses qui exigent un fort soutien de l'administration. Pourquoi? Parce qu'une équipe peut être très conservatrice et jalouse de son territoire lorsqu'on touche à sa manière de faire. D'un certain côté, c'est une très bonne chose, parce que cela veut sans doute dire qu'elle s'approprie les 
services qu'elle rend. D'un autre côté, les personnels peuvent ne pas désirer faire de compromis ni changer quoi que ce soit, surtout si c'est pour faire la même chose que dans d'autres départements ou services. Vous allez certainement entendre des choses du genre: «Nous faisons comme ça depuis plus longtemps qu'eux, pourquoi serait-ce à nous de changer notre manière de faire?»

Pour contrebalancer le repli de chacun sur son territoire et ses habitudes, votre bibliothèque doit être claire sur le fait de s'engager, avant toute chose, pour améliorer l'expérience de l'usager. À partir de là, il ne devrait pas être exagérément difficile de démontrer qu'il est dans l'intérêt des utilisateurs de bénéficier de la même qualité de service et des mêmes repères partout dans la bibliothèque.

\section{Parlez entre vous}

Cela a l'air simple, n'est-ce pas? Si vous encouragez vos agents à se parler avant de prendre des décisions qui ont un impact sur l'offre de services, il y a fort à parier que les incohérences entre services se réduiront rapidement.

\section{Évaluez de manière similaire}

Si vous collectez des statistiques ou lancez une évaluation des services fournis par votre bibliothèque, un premier pas pour rendre vos services homogènes, quel que soit le département ou le type d'accès, est de vous assurer que tout le monde évalue ces services et collecte ces statistiques de la même manière. Cela devrait permettre de prendre des décisions fondées sur des preuves qui pourraient fonctionner quel que soit le département ou le mode d'accès au service et non de manière isolée.

\section{Employez les mêmes outils}

Si vous avez plus d'un département dans votre organisation qui demande aux usagers de s'identifier pour s'inscrire à une formation 
ou à un événement, vous devriez utiliser le même logiciel d'inscription. Cela paraît évident, pas vrai? Et pourtant, il arrive, dans certaines bibliothèques, que des outils ou des logiciels distincts soient utilisés pour faire la même chose. C'est à la rigueur acceptable si ces outils sont cantonnés à du travail interne, mais cela devient inadmissible si vos usagers ont besoin de les utiliser.

\section{Ayez des normes communes de service}

Avez-vous une norme pour les files d'attente aux bureaux d'information (par exemple, un usager ne doit pas attendre plus de trois minutes avant qu'un membre du personnel le prenne en charge)? Y a-t-il une norme pour le temps de traitement des demandes par mail (façon charte Marianne: "Toutes les demandes reçoivent une réponse sous 24 heures») ? Si tel n'est pas le cas, il s'agit de deux des normes (parmi d'autres) de service que vous pouvez mettre en place dans votre bibliothèque. Le plus important? Ne définissez pas ces normes tout seul: faites en sorte que tous les départements de la bibliothèque s'impliquent. Des normes de service cohérentes sont capitales pour améliorer la cohérence globale de la qualité des services dans toute organisation.

\section{Pensez à l'harmonisation}

Vous pourriez envisager d'entreprendre un projet plus large d'harmonisation de tous les services de votre bibliothèque. Ce travail exige une adhésion à tous les niveaux, ainsi qu'un engagement réel de faire des changements dans l'intérêt d'une expérience utilisateur plus homogène. L'effort d'harmonisation des services doit commencer par réunir toutes les parties prenantes qui sont responsables d'une offre de services au public. Une fois ces personnes réunies, l'étape suivante consistera à demander à chaque service de présenter tous les détails de sa prestation au groupe de manière à ce que tout le monde soit sur la même longueur d'onde. Après cela, il sera possible d'inventorier tous les 
services offerts dans la bibliothèque, ainsi que des plates-formes, outils et ressources utilisées pour rendre ces services (systèmes, logiciels, applications, équipement, etc.).

Vous pouvez gagner en cohérence et en efficacité si vous comparez les types de services et les ressources utilisées. Vous pourrez aussi être agréablement surpris par le potentiel d'harmonisation des services lorsque vous faites travailler ensemble les bonnes personnes, dans la même pièce, sur des enjeux communs.

\subsection{Le service est cohérent dans toute l'organisation}

\section{Difficulté ***}

Après ce dernier diagnostic, vous pourriez penser que tout a été dit sur la cohérence, mais ce n'est pas encore le cas. Certes, s'assurer de l'homogénéité entre départements et modes d'accès est crucial. Mais un autre type de cohérence est tout aussi important pour assurer une expérience de haute qualité aux membres de votre communauté.

\section{Pourquoi c'est important}

Une histoire de la vie courante pour expliciter le propos:

Vous faites vos courses dans votre supérette de quartier et vous avez vraiment besoin de mettre la main sur du combava, car votre émission de cuisine préférée a vanté les vertus de cet agrume pour faire un bon rougail. Vous avez fait trois visites au magasin, et demandé à trois personnes différentes s'ils avaient du combava. Les trois employés se sont excusés et vous ont dit que non, ils n'avaient plus de combava depuis un moment et ne savaient pas quand le magasin serait livré. Et de vous laisser sur ces belles paroles. Comment vous sentiriez-vous? Désappointé sans doute. Probablement un peu embêté, car vous allez devoir 
chercher votre combava dans une autre boutique et que vous n'avez aucune garantie d'en trouver.

Si vous aviez à noter le service reçu dans votre supérette à ces trois occasions, vous pourriez dire qu'il a été correct. Cohérent, même, puisque vous avez reçu la même réponse des trois personnes de l'équipe.

Reprenons maintenant ce scénario au moment où le premier employé vous dit que: "Non, nous n'avons pas de combava»; mais cette fois-ci vous demande: "Quelle recette essayez-vous de réaliser? Avez-vous envisagé de remplacer le combava par du citron vert, qui est parfois utilisé comme substitut du combava dans le rougail?» Et si le deuxième employé vous avait dit que: «non, nous n'avons pas de combava, mais je vais aller voir le responsable de rayon pour savoir où en est la livraison de combava»? Et si la troisième employée vous avait dit que: «Non, nous n'avons pas de combava, mais êtes-vous allé voir dans la boutique exotique trois rues plus loin, parce que j'en ai vu là-bas dans la vitrine en passant devant à vélo »?

Maintenant, si vous aviez à noter le service que vous avez reçu de votre supérette à ces trois occasions, que diriez-vous? Vous répondriez sans doute qu'il était vraiment excellent.

Était-il cohérent? Eh bien, non, si être cohérent signifie recevoir la même réponse à chaque fois, de la part de tous les employés. Mais si on vous demandait si le service était constamment au-dessus de la moyenne, vous répondriez probablement par l'affirmative.

Trop souvent, organisations et procédures sont structurées de manière à mettre en œuvre le premier type de cohérence, celui qui s'assure que tous les règlements et procédures sont clairs, que tous les membres du personnel les connaissent, et qu'ils répondent de la même façon lorsqu'un même cas de figure se présente. Une telle cohérence formelle est sécurisante en termes de management, et confortable pour les employés. En réalité, 
c'est vers l'autre type de cohérence, celui qui permet d'offrir un service toujours remarquable, qu'il faut tendre. C'est beaucoup plus difficile à réussir, bien sûr, et il est fort délicat de former des gens pour le faire : mais cela en vaut la peine et permet d'améliorer de manière significative la qualité de l'expérience de vos usagers.

\section{À vous de jouer}

Pour évaluer la cohérence du service dans une organisation, vous ne pouvez pas faire un sondage minute. La cohérence ne peut se mesurer que dans la durée. Voici deux propositions pour évaluer ce point précis dans votre bibliothèque. La première demande un certain investissement et nécessite d'être planifiée; la seconde est un peu plus facile à mettre en œuvre. Choisissez la méthode qui convient le mieux à votre organisation.

\section{Option 1: sondes culturelles (ou enquête ethnographique allégée)}

Les sondes culturelles ont été abordées dans la partie «Méthodes d'analyse des comportements» du chapitre 2. Pour cela, il vous faudra recruter au moins cinq utilisateurs réguliers de votre bibliothèque et leur donner à chacun un carnet de notes pour raconter leurs expériences après cinq visites à la bibliothèque.

Demandez-leur de reporter des informations détaillées à propos de chacune de leurs visites: pourquoi ils sont venus, à qui ils ont parlé, quel service ils ont reçu et leur sentiment sur ce service. Certains usagers pourraient compléter ce travail en une semaine, d'autres prendre un peu plus de temps. Une fois que chacun d'eux vous aura remis son journal de bord, organisez un court entretien avec chacun pour jauger la cohérence du service qu'ils ont reçu. Donnez-vous 3 points pour chaque participant qui vous dit que le service était constamment remarquable (maximum 15 points). 


\section{Option 2: interviews d'usagers}

Si vous n'avez pas assez de temps à consacrer à une enquête ethnographique allégée, vous pouvez sans doute obtenir des résultats similaires, bien que moins riches, en vous contentant de faire les entretiens décrits à la fin du paragraphe précédent. Recrutez au moins cinq visiteurs réguliers et posez-leur des questions ouvertes sur leurs cinq dernières visites. Assurez-vous d'avoir avec vous quelqu'un pour prendre des notes ou, mieux encore, enregistrez les entretiens de manière à pouvoir réécouter les enregistrements et réfléchir à chacune des réponses. Si vous enregistrez des interviews, pensez à demander systématiquement la permission à tous les interviewés. Là encore, donnez-vous 3 points pour chaque participant qui vous dit que le service était toujours remarquable (maximum 15 points).

\section{Passez à l'action}

Il y a peu de manières de faire monter votre cote d'utilisabilité. Si votre recherche sur les usages (enquête ethnographique ou entretiens individuels) vous a révélé que vous n'étiez cohérent ni dans la réponse (tout le personnel ne donne pas les mêmes réponses), ni en termes de niveau de service, et que vous ne retrouvez aucun signe indiquant que le service est apprécié pour sa grande qualité, reprenez ce chapitre au début et travaillez sur les exercices suggérés pour passer à l'action. Si vous réussissez bien en matière de cohérence de la réponse (tout le monde dit la même chose), mais que votre score sur le niveau de service est moins bon, c'est bien. Vous êtes à mi-parcours, ne vous arrêtez pas en si bon chemin! Les idées suivantes vous permettront de surmonter les derniers obstacles.

Recommencez encore et encore! Il s'agit du dernier point de ce chapitre, pour une bonne raison: il n'est pas possible que vous 
puissiez rendre de manière constante un service exceptionnel à moins d'avoir travaillé tous les points qui précèdent.

Assurez-vous:

$\odot$ d'avoir élaboré une philosophie des services;

$\odot$ que vos personnels la connaissent et la fassent vivre;

$\odot$ d'avoir réduit le nombre de règlements et de procédures;

$\odot$ de donner du pouvoir à vos agents et de renforcer leur empathie envers les différents publics à desservir pour avoir l'équipe la plus accueillante et la plus prévenante possible.

\section{Un engagement suivi dans la durée}

Si vous avez introduit les changements dont nous avons parlé dans ce chapitre afin d'améliorer le service rendu dans votre bibliothèque, c'est bien!

Mais sachez que vous ne pouvez pas vous en féliciter et tourner les talons pour passer à autre chose. Ces choses-là exigent un entraînement suivi et des piqûres de rappel. Considérez qu'il faut répéter la plupart des exercices dont nous avons parlé dans ce chapitre (le client mystère, la liste des "Non », l'enquête contextuelle, les sondes culturelles) de manière suivie. Assurez-vous également que vous motivez vos agents de manière régulière, en les encourageant à s'investir dans les exercices de suivi autant que possible, et en communiquant les résultats de ces exercices de manière cohérente.

\section{Envisagez une rotation des personnels}

Il est assez facile de perdre son énergie et sa vigueur en service public lorsqu'on fait le même travail, au même bureau d'accueil, dans la même bibliothèque, tous les jours, depuis des années. 
Si vous avez un peu de souplesse pour proposer plus de mobilité aux personnels d'accueil au sein de l'établissement, envisagez de le faire. Qu'il s'agisse de déplacer les gens entre "sections» ou juste de les faire bouger entre bureaux d'accueil dans un même département, changer les choses tous les 12 ou 24 mois environ peut faire des merveilles pour éviter aux agents de s'ennuyer et de perdre leur empathie. Le point positif pour la bibliothèque est d'avoir un personnel d'accueil parfaitement polyvalent entre départements, ce qui vous permet plus de souplesse et d'agilité pour des changements plus ambitieux. 


\section{Chapitre 6. Signalétique et orientation}

Où suis-je? Que puis-je faire ici? Où puis-je aller à partir d'ici? Où sont les toilettes? Comment puis-je sortir d'ici? Où se trouve ce livre? Comment trouver la bonne direction?

Dans l'idéal, la signalétique de votre bibliothèque doit permettre aux gens de répondre à toutes ces questions. Elle est une aide essentielle, notamment pour les nouveaux arrivants. Une signalétique plus ou moins réussie peut non seulement améliorer ou diminuer la qualité de l'expérience visuelle dans votre bibliothèque, mais aussi jouer sur la probabilité que les gens y vivent un moment agréable.

Imaginez la signalétique de votre bibliothèque comme une forme de service public, comme la traduction de son attitude envers ses utilisateurs. À l'instar du personnel d'accueil, est-elle agréable, utile et prête à aider ceux qui en ont besoin? La signalétique permet de partager des informations avec les gens: il faut donc bien la concevoir comme faisant partie intégrante du travail de la bibliothèque. Vous pourrez utiliser les diagnostics de ce chapitre pour évaluer et améliorer les différentes facettes de la signalétique dans votre établissement.

\subsection{Votre bibliothèque dispose d'une charte graphique bien conçue}

\section{Pourquoi c'est important}

\section{Difficulté $* * *$}

Une charte graphique, au sens large, est une liste de préconisations qui indiquent à quoi la signalétique (murale, imprimée, en 
ligne...) devrait ressembler. En ce sens, une charte graphique est intrinsèquement prescriptive. Développer un tel outil prend du temps, mais, en fin de compte, sa mise en place permettra d'en gagner, car vous ne partirez pas de zéro à chaque fois que vous aurez besoin de créer une nouvelle signalétique ou une nouvelle page web. Les décisions concernant la forme et le design auront déjà été prises, et créer un nouveau support sera aussi simple que de mettre à jour un contenu déjà stylé.

Cette charte devrait être utilisée pour tout ce que voient les usagers de la bibliothèque et tout ce qui leur est distribué: brochures, affiches, site web, cartes de lecteurs, signalétique, sacs fourre-tout, etc. Pourquoi est-ce important? Une identité visuelle cohérente rendra votre établissement attractif et reconnaissable et permettra au public de savoir où il met les pieds. Cette identité graphique vous permettra d'exprimer de manière visible les valeurs portées par votre établissement, sa personnalité. Cette identité montrera aussi que vous êtes attentif aux détails, ce qui augmentera le degré de confiance que le public place dans votre bibliothèque. Les bibliothécaires sont des professionnels de l'information et, à ce titre, devraient traiter leurs informations en conséquence. Bien que cela puisse aider, vous n'avez pas besoin de devenir un expert en design ou un graphiste chevronné pour créer une charte graphique pour votre établissement. Les étapes suivantes vous permettront de démystifier le processus de création graphique.

Dans de nombreux cas, vous êtes dépendant de l'identité graphique de votre établissement de tutelle, collectivité territoriale, université ou école. Appréciez votre marge de manœuvre et ce qui peut permettre à la bibliothèque d'être bien identifiée dans cette communauté: votre propre jeu de couleurs, un complément de logo, des éléments de langage ou un slogan en propre, une police complémentaire. De toute manière, les informations ci-dessous peuvent vous permettre d'ouvrir la discussion avec les services de communication, de mieux comprendre la logique de leur travail 
et de vous appuyer sur un vocabulaire commun. Souvent, pouvoir s'appuyer sur une identité visuelle construite par des professionnels est une chance et non une contrainte: engagez le dialogue sur ce qui fait la spécificité de votre bibliothèque et sur la façon dont elle peut s’inscrire dans une démarche générale.

\section{À vous de jouer}

Soit votre établissement dispose d'un référentiel, soit il n'en a pas. L'autodiagnostic est facile à faire.

Vous avez une charte graphique complète et vous l'utilisez: 25 points.

Vous n'en avez pas: 0 .

Vous êtes à un stade intermédiaire? Attribuez-vous des points comme bon vous semble. Souvenez-vous, le CUTI n'est pas une science exacte, tant que vous faites preuve d'honnêteté intellectuelle et d'esprit critique.

\section{Passez à l'action : construisez une charte graphique}

Pour créer cette charte graphique, vous choisirez des polices de caractère et des couleurs officielles, propres à votre établissement, et fournirez un guide d'usage indiquant comment ces éléments doivent être mis en œuvre. Voici un exemple des éléments indispensables à la mise en place d'une charte graphique digne de ce nom.

Éléments de base:

1. Typographie - précise les polices de caractère qui devront être utilisées sur tous les supports imprimés, des logos aux titres, en passant par le texte;

2. Couleurs - donnent toutes les informations sur les couleurs principales et secondaires qui devront être utilisées sur l'ensemble des supports; 
3. Logo ou marque - règle d'usage, emplacement et modèles couleur et noir et blanc intégrables dans des documents web ou destinés à l'impression sur papier (vectoriel).

Gabarits type pour différentes sortes de documents:

4. Signalétique;

5. Affiches;

6. Brochures;

7. Site web.

Voici quelques éléments dont vous devrez tenir compte lorsque vous définirez votre charte graphique.

\section{Sélection des polices de caractère}

La base de la communication visuelle repose sur les lettres. Ces lettres peuvent être de taille et de formes variées: choisir les meilleures pour votre bibliothèque est un enjeu important en termes de design. Sélectionner les bonnes polices de caractère va au-delà du choix de quelque chose de «joli»: vous devez également tenir compte de la lisibilité d'une police et de sa capacité à transmettre les valeurs de votre établissement. Choisissezles avec soin, car vous les utiliserez dans toutes les productions de votre bibliothèque. Restez-en aux polices classiques: elles ne le sont pas devenues sans une bonne raison. Les polices peuvent être regroupées en deux grandes familles:

Serifs: les polices à empattement ont de petites extensions au bout des lettres. Ces extensions sont appelées serif en anglais, traduit par empattement en français. Elles donnent souvent aux caractères une dimension formelle, élégante et traditionnelle.

\section{Bodoni Garamond Times Century}


Dans la plupart des cas, les polices à empattement sont intéressantes pour les longs paragraphes. De nombreux livres sont imprimés dans ce type de police. Les polices à empattement les plus populaires sont Georgia et Times.

Sans empattement ou sans serif: comme leur nom l'indique, les polices sans empattement sont dépourvues des extensions ornementales au bout des caractères. Les polices de ce type peuvent donner au graphisme une apparence moderne, élégante et chaleureuse. Les plus courantes sont:

\section{Helvetica Frutiger Futura Gotham}

Elles sont particulièrement efficaces quand elles sont utilisées pour les titres ou la signalétique. Observez la signalétique de n'importe quel aéroport, vous pouvez être sûr de trouver une police sans empattement. Notez que si les polices avec empattement sont adaptées aux longs textes imprimés, celles qui en sont dépourvues sont plus lisibles sur la plupart des écrans.

Les polices les plus populaires adaptées à la lecture sur écran sont Arial et Verdana.

Combien de polices choisir? Probablement deux. Les polices que vous aurez sélectionnées ont besoin de bien fonctionner ensemble: en choisir plus de deux compliquera leur combinaison. Une valeur sûre consiste à associer une police avec empattement avec une qui en est dépourvue. Cela garantit un bon contraste entre les deux.

L'exemple suivant associe un titre en Bodoni avec un corps de texte en Helvetica: 
Agréables et classiques tout à la fois, ces deux polices sont simples et lisibles.

Une autre bonne combinaison consiste à associer un titre en Helvetica avec un corps de texte en Century Schoolbook.

\section{Library News}

Come to the library on June 6 th for face painting. Kids love this for some reason.

Dans les deux exemples qui suivent, remarquez comment le titre en Futura Bold (à droite) offre une hiérarchie visuelle plus forte et, en règle générale, fonctionne mieux avec un corps de texte en Helvetica qu'un titre en Futura Light (à gauche).

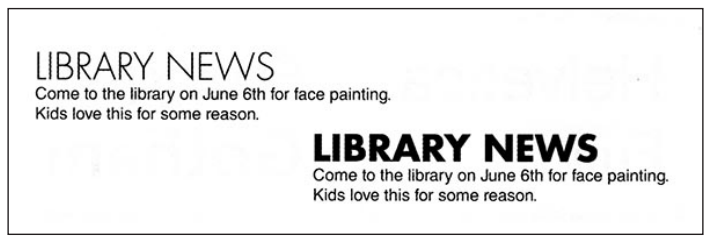

Trop simple? Choisir une seule police peut conduire à des graphismes inélégants, lourds ou difficiles à lire. Si vous n'en utilisez qu'une seule, essayez Helvetica et, dans ce cas, veillez à augmenter les contrastes afin de souligner le caractère délibéré de votre graphisme. L'exemple qui suit met en contraste un titre en Helvetica Bold avec un corps de texte en Helvetica:

\section{Library News}

Come to the library on June 6th for face painting. Kids love this for some reason.

Plusieurs exemples ci-dessus mettent en œuvre des polices payantes comme Helvetica ou Futura, qui ne sont pas fournies par défaut dans les logiciels de traitement de texte de base. Si vous faites le choix d'adopter une police payante, ce qui vaut souvent 
le coup et assure à votre communication visuelle un aspect professionnel, vous veillerez à la faire installer sur tous les postes.

\section{Mettez en place un référentiel de couleurs}

Le spectre des couleurs est infini: choisir les bonnes pour votre bibliothèque peut vous paraître une lourde responsabilité. À l'instar des polices que vous aurez choisies, vous aurez intérêt à utiliser partout ces couleurs. Quand vous choisissez des couleurs, gardez toujours à l'esprit qu'elles seront utilisées sur de multiples supports, imprimés ou en ligne. Limitez-vous à une palette de couleurs simples, en visant peut-être quatre à cinq couleurs (hors noir et blanc). En intégrer davantage rendra non seulement le choix plus difficile, mais compliquera leur agencement.

Avant de trouver de jolies couleurs, il vous faut savoir ce que vous voulez qu'elles expriment. Ces couleurs diront quelque chose de votre établissement: soyez sûr d'envoyer le bon message. Rattachez cette décision aux missions de votre bibliothèque et à son orientation générale. Choisissez trois mots et cherchez des couleurs qui expriment ces valeurs. Par exemple:

$\odot$ fiable, stable, solidaire;

$\odot$ fun, moderne, aventureuse;

$\odot$ à la pointe, drôle, étonnante.

Une excellente manière d'associer des couleurs à ces valeurs consiste à créer ce que les designers appellent un mood board, qu'on pourrait traduire en tableau d'humeurs. Accrochez trois feuilles de paperboard et notez-y les valeurs que vous aurez choisies. Mettez une pile de vieux magazines, de la colle et des ciseaux à proximité et demandez aux usagers de découper les couleurs qu'elles associent à chaque valeur. Mieux encore: disposez tout cela dans un espace public et faites-le faire aux bibliothécaires. Il peut être très intéressant de faire réaliser cet 
exercice en parallèle par le personnel et le public et de comparer les similarités et les différences.

Une fois que vous aurez une idée générale des couleurs que vous souhaitez utiliser, vous n'aurez pas à partir d'une page blanche pour effectuer vos choix finaux. Vous pouvez toujours lancer une recherche de "palette de couleurs» sur le Web et trouver des sites utiles pour vous lancer. Jetez un œil sur des sites comme COLOURlovers < www.colourlovers.com > et Adobe Kuler $<$ https://kuler.adobe.com >. Ils vous donneront de l'inspiration et vous aideront à choisir des couleurs fonctionnant bien ensemble.

Gardez une trace de votre nuancier en enregistrant chacun des codes hexadécimaux (\#8CCCD9, par exemple) et, si vous produisez beaucoup de documents imprimés, leurs références Pantone, entreprise américaine dont les nuanciers font référence dans le monde entier (par exemple, Pantone 630 - Vintage Blue) ou RVB, Rouge Vert Bleu, système de codage informatique des couleurs (par exemple, 140, 204, 217).

\section{Créez votre logo}

Prendre au sérieux l'expérience globale de l'utilisateur, ainsi que l'image de votre bibliothèque et la relation entre les usagers et votre établissement, implique d'embaucher un professionnel pour en réaliser le logo (un symbole qui représente votre bibliothèque) ou la marque (un type de logo qui n'est jamais qu'une représentation textuelle de votre établissement). Vous pouvez également mobiliser de futurs professionnels en formation pour explorer des pistes, dès lors que vous vous engagez à ne pas utiliser leur travail sans rémunération.

Si vous n'avez pas les moyens de recruter un professionnel, il faut envisager de concevoir votre logo ou votre marque vous-même. Investissez-y un peu de réflexion, ils n'en seront que mieux conçus. Avant de commencer la conception, recherchez des sources 
d'inspiration dans les logos des grandes marques. Le travail de Saul Bass (graphiste américain, auteur notamment de génériques et d'affiches de films célèbres), PaulRand (graphiste américain, auteur par exemple du logo d'IBM) et de Chermayeff \& Geismar (auteurs des logos d'entreprises comme NBC, Mobil...) est un bon point de départ. Consultez la bibliographie en fin de chapitre pour des sources d’inspiration complémentaires.

Dressez ensuite la liste de certaines des caractéristiques de votre bibliothèque et de la communauté qui l'entoure. Y a-t-il des particularités architecturales ou naturelles exceptionnelles pouvant servir de base à un logo? Gardez à l'esprit que vous avez déjà sélectionné vos polices de caractère et vos couleurs de référence. Pouvez-vous les utiliser pour produire quelque chose d'efficace et approprié?

Prenez soin d'utiliser un logiciel de dessin vectoriel tel qu'Adobe Illustrator ou ses équivalents libres comme Inkscape ou OpenOffice Draw pour créer votre logo. Cela vous permettra de l'adapter à divers usages, y compris les formats d'impression en haute résolution, et de les redimensionner sans dégrader la qualité de l'image.

Comme dans tous les domaines du design, la simplicité produira la plupart du temps de bons résultats en limitant les risques d'impair. Si vous n'êtes pas satisfait de ce que vous avez créé, optez pour le nom de votre bibliothèque en utilisant l'une des polices que vous aurez choisies.

\section{Plainville Library}

\section{Concevez des gabarits}

Sélectionner des typographies et des couleurs est un bon début, mais il y a encore du travail à faire. L'étape suivante - créer des gabarits - orientera la mise en œuvre de ces éléments et 
garantira que les typographies et couleurs que vous aurez choisies seront utilisées de manière appropriée.

Avant de concevoir ces gabarits, vous devez savoir ce que vous voulez. Si vous ne l'avez jamais fait, c'est le bon moment pour lancer un audit sur la signalétique de votre bibliothèque (6.2 Une même charte graphique pour toute la signalétique). Vous trouverez probablement de nombreux éléments de signalétique exigeant un design propre, mais complémentaire (6.3 Les différents types de signalétique sont visuellement distincts). Une fois que vous aurez dressé cette liste pour votre bibliothèque, vous saurez quels types de gabarits créer.

Pour illustrer cet exemple, nous créerons un gabarit de format $21 \times 29,7$ pour une affiche d'information, et montrerons comment il peut être utilisé.

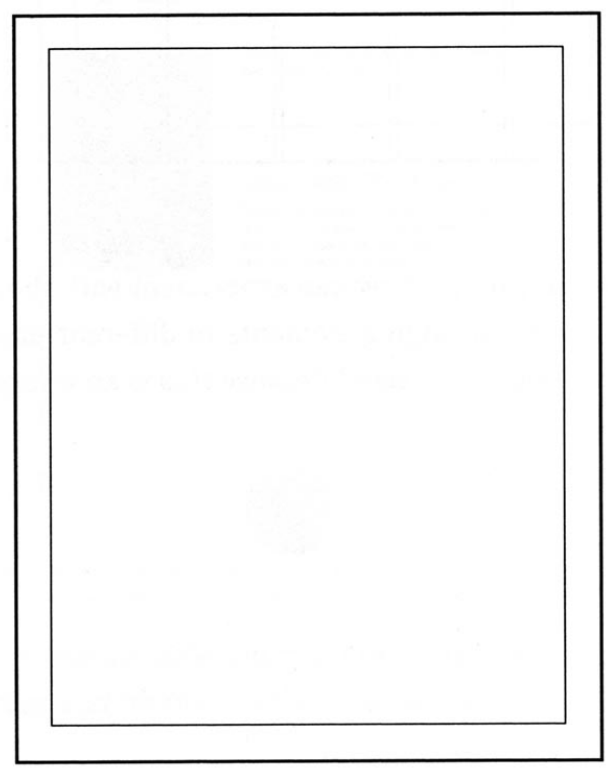

Fig. 6.1 Gabarit pour une affiche d'information 


\section{Disposition}

Créer des gabarits structurés par une grille vous simplifiera la vie et contribuera à homogénéiser l'ensemble de votre production. La grille désigne le découpage en colonnes qui facilite le placement du texte, la mise en place d'une hiérarchie visuelle et vous aide à structurer la conception globale.

Avant de diviser le canevas, il est nécessaire de créer une bordure, l'ensemble de l'espace n'étant pas utilisable. Insérer du contenu - plus particulièrement du texte - sur le bord de votre canevas rendra les choses difficiles à lire et ajoutera de la tension dans le design. Créez donc une bordure au sein de laquelle vous insérerez le texte. Dans l'exemple suivant, la page est entourée de chaque côté d'une marge d'un centimètre.

Pressé par le temps ? S'il est recommandé de créer un gabarit pour chaque type de signalétique dans votre bibliothèque, les contraintes de temps limitent ce qu'il est possible de faire. N'abandonnez pas pour autant. Disposer d'un unique gabarit bien conçu pour l'ensemble de la signalétique de votre établissement sera toujours mieux que de n'en avoir aucun. Qui plus est, il vous sera plus facile de créer un consensus avec un seul gabarit et d'inciter ainsi vos collègues à l'utiliser.

Créer une grille implique de diviser l'espace utile du canevas. Bien que les manières de diviser cet espace soient infinies, nous choisirons ici, pour faire simple, cinq colonnes assez larges. Il s'agit d'un nombre pertinent, car il permet de limiter les variables et les complications inutiles. Mais créer une grille est un peu plus complexe que la simple division de l'espace en parts égales. En plus des marges entourant le gabarit, il est nécessaire de laisser des espaces de respiration entre les colonnes, appelés 
gouttières (figure 6.2). Sans gouttières, les éléments de la page se chevauchent et sont illisibles.

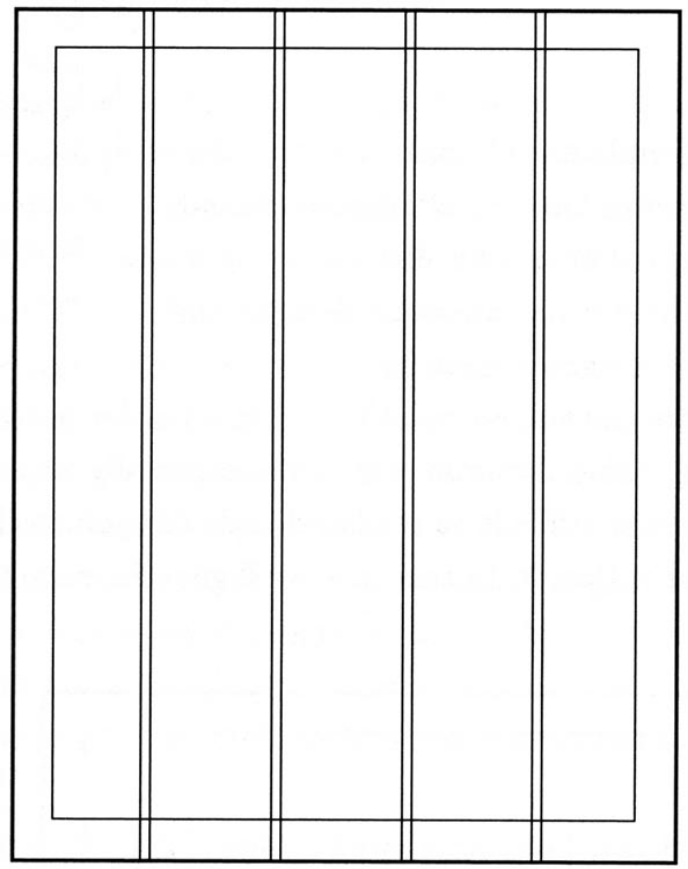

Fig. 6.2 Grille et gouttières

Une fois le canevas obtenu, on peut tester la disposition des éléments de signalétique informationnelle en variant leur agencement sur le gabarit. Mais tout d'abord, quels sont les contenus devant figurer dans notre signalétique? Les informations de base incluent:

$\odot$ le logo de la bibliothèque;

$\odot$ la nature de l'événement;

$\odot$ la date de l'événement;

$\odot$ le lieu de l'événement.

La figure 6.3 en donne un aperçu. Faites des essais en plaçant ces informations élémentaires à différents endroits de la grille, comme dans la figure 6.4. 


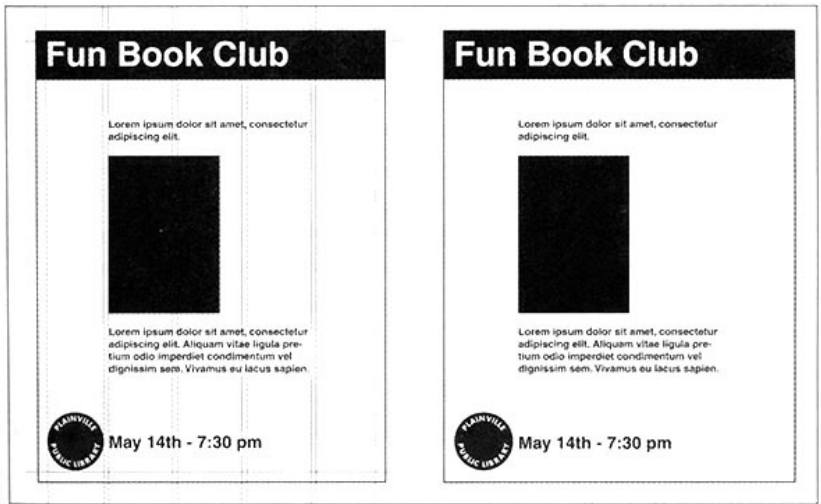

Fig. 6.3 Placement des éléments sur la grille

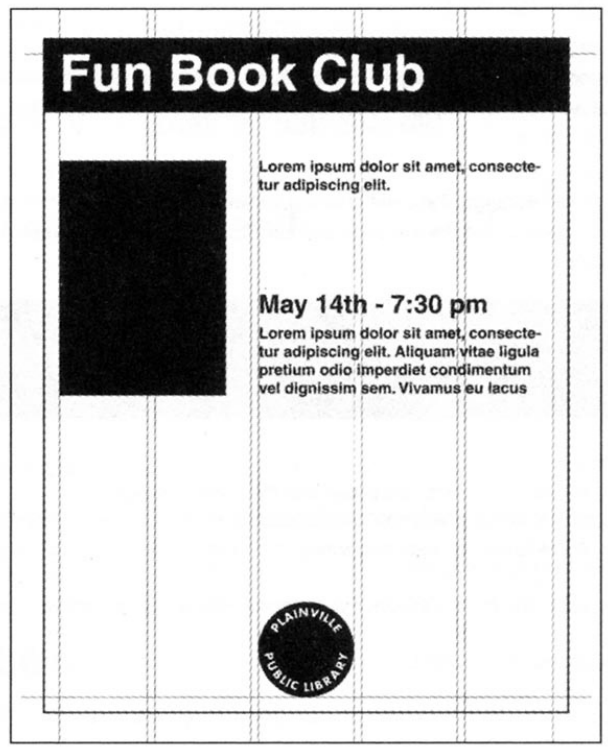

Fig. 6.4 Les éléments de la figure 6.3 retravaillés

Créer des gabarits pour les différents types de signalétique de votre établissement facilitera leur production et, grâce à une conception cohérente, les rendra plus facilement compréhensibles pour vos utilisateurs. 
Une bibliothèque de modèles pour votre site web

Votre site web devrait employer les mêmes typographies et couleurs que vos autres productions graphiques. Là encore, utiliser les mêmes typographies et couleurs ne suffit pas à garantir un design réussi. Vous aurez également besoin de structurer la disposition de vos pages web. Une manière efficace de le faire est de créer une bibliothèque de modèles. Celle-ci est un peu comme un gabarit, mais, au lieu - ou plutôt en plus - d'indiquer exactement comment disposer les éléments sur la page, cette bibliothèque détaille l'aspect qu'ils devraient prendre. Même si le design du site est réalisé par une seule personne, ce sera toujours un outil très utile. Le site web de démonstration du Global Experience Language de la BBC < http://static.bbci.co.uk/gel/0.5.7/downloads/GEL_web_styleguide.pdf > est un exemple assez remarquable. Une autre excellente bibliothèque de modèles est celle de la Grand Valley State University (<http://labs.library.gvsu.edu/ ui/ > et figure 6.5).

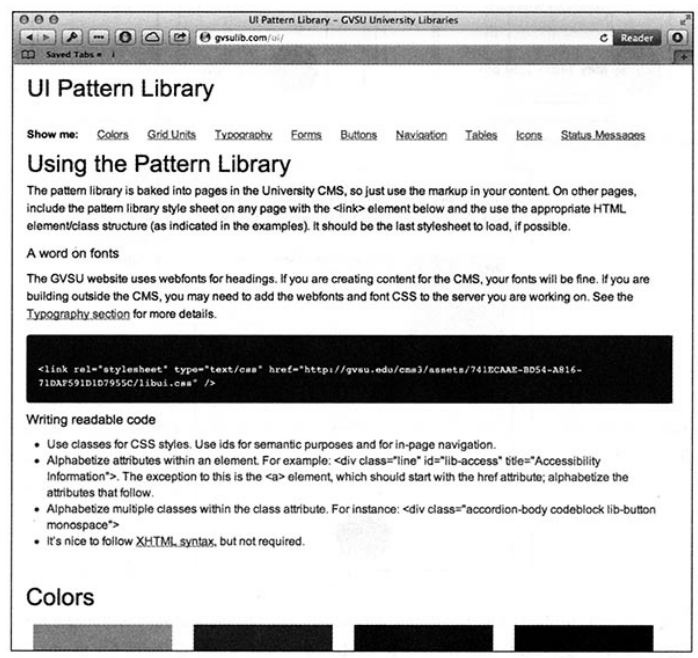

Fig. 6.5 Bibliothèque de modèles de la Grand Valley State University 
Créez un gabarit pour tous les types de page de votre site. Des pages type peuvent être, par exemple:

$\odot$ la page d'accueil;

$\odot$ les pages de renvoi ou de navigation;

$\odot$ les catégories et sous-catégories;

$\odot$ les formulaires.

Détaillez ensuite l'aspect que devraient prendre les différents éléments sur ces pages. Développer une bibliothèque de modèles fait gagner du temps sur le long terme et est essentiel dans tout projet de refonte de site web.

Utilisez les couleurs efficacement. Gardez ces différents points à l'esprit quand vous concevez vos gabarits: vous créerez une hiérarchie cohérente si un même type de signalétique correspond toujours à la même couleur; le noir est difficile à lire sur la plupart des couleurs. Restez-en au noir et blanc uniquement, ou peut-être au jaune. Avez-vous déjà remarqué que la plupart des aéroports utilisent du noir sur fond jaune pour les informations importantes? Le noir et le jaune forment un contraste saisissant, facile à lire pour la plupart des gens, même à distance; un texte coloré peut être attrayant, mais aussi difficile à lire. Le choix le plus sûr est d'oublier les typographies en couleur et d'en rester, pour le texte, au noir et blanc; un texte en blanc est un choix pertinent sur n'importe quelle couleur, pourvu qu'elle soit assez sombre pour produire un contraste suffisant.

\section{Mettez en ouvre dans la durée} et partout votre nouvelle identité visuelle

Même si vous suivez scrupuleusement le mode d'emploi ci-dessus pour établir votre nouvelle identité visuelle (police, couleurs, 
logo, gabarit), vous ne pourrez pas l'implémenter d'un seul coup de baguette magique partout où il le faudrait dans votre bibliothèque.

Pour y arriver, vous devrez d'abord faire l'inventaire de tous les endroits où vous utiliserez cette nouvelle identité visuelle - des marque-pages au site web-, puis créer un plan d'action et d'implantation réaliste et le respecter. Des progrès mesurables et constants sont, en la matière, une bonne chose. Un plan d'implantation à moitié réalisé et abandonné à mi-chemin est inefficace. Si vous n'allez pas jusqu'au bout de la démarche, votre gros effort initial de création d'une charte graphique n'aura servi à rien.

\subsection{Une même charte graphique pour toute la signalétique}

\section{Difficulté **}

\section{Pourquoi c'est important et à vous de jouer}

Cela devrait être évident: la meilleure charte graphique du monde ne donnera rien si elle n'est pas mise en œuvre correctement sur le terrain.

Regardez chaque élément de la signalétique de votre bibliothèque. Est-il conforme à votre charte graphique? Identifiez tout ce qui n'en relève pas et corrigez-le. Vous pouvez pour ce faire conduire un audit de la signalétique et, en la matière, la méthode la plus simple et la plus rapide de procéder est de prendre des photos. Insérez-les dans un tableur et listez les points suivants:

$\odot$ localisation de la signalétique;

$\odot$ nature de la signalétique - directionnelle, d'identification, pédagogique, informative, réglementaire (6.3 pour la définition de ces termes); 
$\odot$ conforme à la charte graphique? (oui ou non);

$\odot$ scotché au mur? (oui ou non);

$\odot$ rédigé de manière correcte? (oui ou non).

N'oubliez pas que pour atteindre les 15 points vous devez, ici, disposer d'une charte graphique. Si, après l'audit, vous constatez que l'ensemble de votre signalétique s'inscrit dans l'identité visuelle de la bibliothèque décrite dans la charte: 15 points.

Si vous trouvez beaucoup d'éléments aberrants ou incohérents: 0 point. Comme toujours des notes intermédiaires sont possibles, mais ne soyez pas trop indulgent.

\section{Passez à l'action et normalisez votre signalétique}

L'audit de la signalétique vous permettra de planifier votre travail pour supprimer les éléments hétérogènes ou aberrants. Ne vous débarrassez pas de votre fichier une fois que vous aurez terminé! Utilisez-le comme un outil d'évaluation permanent, vous permettant de garder une trace de toute la signalétique qui aura été créée.

Ce plan de travail suppose bien sûr que vous ayez déjà créé votre charte graphique. Si c'est le cas, bravo! Supprimer la signalétique inadaptée sera aussi simple que de la rendre compatible avec les lignes directrices de votre charte. Si vous n'en avez pas encore, laissez cette étape en suspens. Une fois que vous aurez une identité visuelle, vous pourrez y revenir.

\subsection{Les différents types de signalétique sont visuellement distincts}

\section{Pourquoi c'est important}

\section{Difficulté $* * *$}

Il y a différentes sortes de signalétique dans les bibliothèques et toutes ont des objectifs différents. Certaines, par exemple, 
permettent d'aider les gens à se rendre d'un point à un autre; d'autres les informent d'un changement dans le règlement. Quand chaque élément a son propre design visuel, c'est tout le système de signalétique qui gagne en efficacité. C'est une bonne chose en soi, mais il faut surtout garder à l'esprit le résultat final: ce système aidera les usagers de la bibliothèque à l'utiliser avec succès.

\section{À vous de jouer}

Avant de veiller à ce que les éléments de signalétique de votre bibliothèque soient visuellement distincts, il vous faudra faire le point sur les différentes sortes de signalétiques existantes. En voici une liste, tirée du livre Interior Design for Libraries, de Carol Brown:

Directionnelle: signalétique aidant les usagers à aller d'un point A à un point B. L'exemple courant est celui d'un lieu associé à une flèche.

Identification: signalétique identifiant les zones de la bibliothèque.

Pédagogique: signalétique guidant les usagers dans leur utilisation de la bibliothèque. L'exemple type de cette signalétique est celle installée à proximité des photocopieurs et imprimantes.

Injonctive ou réglementaire: signalétique exprimant une règle ou essayant d'influencer les comportements. De nombreuses bibliothèques ont une signalétique interdisant l'utilisation des téléphones portables ou le fait de consommer de la nourriture.

Informative: signalétique décrivant la bibliothèque ou ses services. Cela va du nom de la bibliothèque sur le fronton du bâtiment à l'annonce d'un événement à venir. Les étiquettes sur les 
livres constituent une signalétique d'identification très présente et parfois négligée. Elle demande un choix cohérent de couleurs et de polices dans la durée et d'éviter la multiplication des pastilles et autres gommettes qui ne parlent qu'aux bibliothécaires et polluent l'espace visuel.

Reprenez la liste de toute la signalétique si vous l'avez faite au moment du diagnostic précédent (6.2) et indiquez de quelle catégorie relèvent ses éléments. Notez également si la signalétique utilise le même design que les autres éléments du même type. Si votre bibliothèque ne dispose pas d'un système de signalétique formalisé, il y a de grandes chances que les éléments ne s'harmonisent pas entre eux.

\section{Passez à l'action et créez des éléments de signalétique visusellement distincts}

Rappelez-vous que chaque élément de signalétique devrait avoir son propre design tout en partageant une même identité visuelle. Bien qu'il ne soit pas facile de créer cette identité dans votre bibliothèque, l'effort initial simplifiera la création d'éléments visuellement distincts. Une signalétique injonctive pourra partager, par exemple, la même typographie qu'une signalétique pédagogique, tout en étant de couleur différente.

Un schéma homogène peut faire évoluer une collection bigarrée d'éléments de signalétique vers un système complet d'orientation. Utiliser des formes et couleurs différentes est une manière efficace d'y arriver. L'ensemble d'une signalétique injonctive pourra ainsi être rouge, blanche, noire et ronde. Tous les signes liés à l'identification pourront être jaunes, noirs et rectangulaires. Il faudra que cela soit plus détaillé, bien sûr: toutes ces spécifications devront être incluses dans votre charte graphique. 


\subsection{La signalétique est minimale}

\section{Pourquoi c'est important}

\section{Difficulté *}

Les éléments de signalétique ne sont pas mauvais en eux-mêmes, mais ils traduisent parfois, par leur abondance, un problème plus général. Pour inverser la perspective d'une façon schématique, un bâtiment idéal n'aurait a priori pas besoin de signalétique directionnelle. Il serait organisé de manière si intuitive que les utilisateurs pourraient s'orienter facilement juste en regardant autour d'eux. De même, dans l'idéal, l'ensemble de vos services et de vos équipements devrait être assez facile à utiliser pour qu'il n'y ait pas besoin, non plus, de signalétique didactique.

Une bibliothèque ainsi conçue et équipée serait la bibliothèque idéale. Elle n'existe pas. Toutes les bibliothèques ont besoin d'une signalétique pour aider les visiteurs à s'orienter et à travailler efficacement. Mais cela ne signifie pas qu'il faille afficher toute la signalétique possible et imaginable... Tout comme vous devez supprimer de votre site web les contenus parasites et les gadgets afin de faciliter la recherche, vous ne devez avoir aucune signalétique superflue dans votre bâtiment. Une signalétique foisonnante distrait l'attention et crée une atmosphère étouffante. Chaque élément ajouté rend les autres moins efficaces.

L'évaluation est toujours ici un peu subjective, car il est difficile de distinguer une signalétique pertinente de celle qui ne l'est pas. Accordez-vous de 0 à 10 points en fonction de la manière dont la signalétique est perçue en général.

\section{Passez à l'action et supprimez la signalétique superflue}

Quels éléments de signalétique devriez-vous supprimer? Commencez par la signalétique injonctive - celle qui influe sur 
les comportements, parce que, la plupart du temps, elle tente de réguler des comportements exceptionnels. Vous obligez ainsi tout le monde à voir quelque chose qui ne s'applique finalement qu'à un très petit nombre de gens. En outre, cette signalétique est la plupart du temps inefficace. Est-ce qu'une simple affiche indiquant aux personnes qu'elles ne sont pas autorisées à consommer de la drogue dans les toilettes va permettre d'éviter ces comportements problématiques? Ils doivent être traités de vive voix, avec prudence et courtoisie, pas avec des affiches.

Une fois la signalétique injonctive supprimée, faites le point sur la signalétique didactique de votre bibliothèque. Que pouvez-vous faire pour la rendre superflue? Il est probable que cela exige des changements substantiels partout où les choses restent confuses pour les usagers. La figure 6.6 illustre bien ce genre de cas de figure.

Supprimer la signalétique directionnelle au jugé n'est pas une bonne idée, car il est difficile de savoir quand elle est utile ou non. Livrez-vous plutôt au test décrit en 6.8 afin de déterminer ce qu'il est pertinent de maintenir.

Après avoir nettoyé l'environnement visuel de votre bibliothèque de sa signalétique inutile, soyez attentif aux changements dans le comportement des usagers. Il est peu probable que vous observiez une augmentation soudaine des infractions ou des comportements déviants, et vous n'aurez probablement pas à répondre vous-même à une série de questions à laquelle cet affichage apportait des réponses.

\subsection{Bannir le scotch}

\section{Difficulté *}

\section{Pourquoi c'est important}

Une affiche scotchée au mur, sur un bureau d'accueil ou sur une table est déprimante. Mais ne pensez pas à vous; préoccupezvous de vos usagers. Cet affichage d'aspect fort peu professionnel 


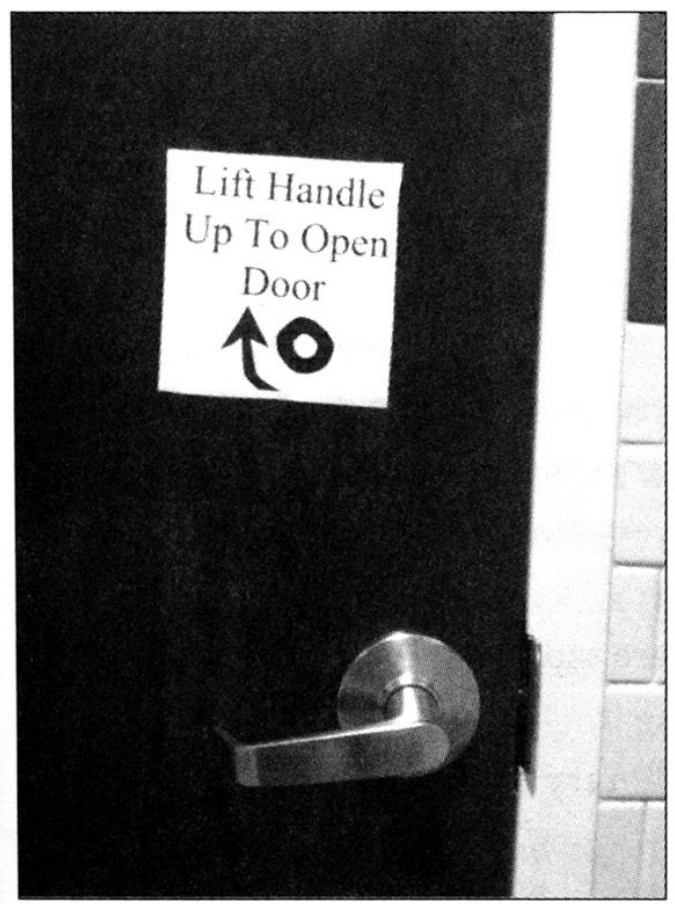

Fig. 6.6 Le signe du bouton de porte

a un effet négatif sur l'environnement visuel de votre établissement et nuit à l'expérience vécue par l'utilisateur. Réfléchissant à l'implication dans l'aménagement de la bibliothèque centrale de Seattle, Bruce Mau écrivait:

Au départ, il y avait un problème, les livres, et une solution, les étagères. Aujourd'hui, quand vous entrez dans une bibliothèque, il y a littéralement des centaines d'affiches et de meubles divers destinés à présenter autant de nouveaux supports. Tout, depuis les magazines jusqu'aux DVD, dispose d'un meuble spécifique, d'un manuel d'utilisateur, d'un catalogue et d'étiquettes spécifiques. Le résultat est un colossal problème de communication. Si l'on peut féliciter les bibliothécaires pour leur inventivité, les usagers, eux, sont confrontés à une confusion permanente, et une superposition organisée de 
strates d’informations scotchées les unes sur les autres. Il est temps d'imaginer autre chose ${ }^{1}$.

Au mieux, ces affichages sont peu attractifs. Après quelques mois de passage et de manipulation, ils donnent un sentiment d'usure et de négligence. Ce n'est certes pas ce que vous aviez en tête, n'est-ce pas?

Dégageant une impression d'amateurisme, ces affiches posées à la hâte sont souvent le symptôme d'une pauvreté de l'aménagement et, peut-être, d'habitudes centrées sur les besoins des bibliothécaires. Généralement, elles sont mises en place parce que quelque chose ne fonctionne pas très bien. Quelques exemples, en vrac:

$\odot$ des instructions pour l'utilisation des bornes de prêt sont scotchées à proximité de l'écran parce que le personnel ne souhaite pas se déplacer pour donner des explications;

$\odot$ une signalétique injonctive est mise en place parce que la bibliothèque n'a pas réussi à trancher entre des besoins contradictoires de lieux de travail en groupe et d'espaces de travail individuel;

$\odot$ un affichage directionnel provisoire est nécessaire pour guider les participants vers leur salle de réunion, car la signalétique permanente est absente ou inadaptée.

Si vous avez déjà fait l'audit proposé en 6.2, vous pouvez mesurer rapidement si votre bibliothèque est parasitée par ces affichages ou non.

S’il n'y a pas d'affichages parasites: 10 points.

1. Bruce Mau, Life Style, New York, Phaidon, 2000, p.242. Bruce Mau est un designer canadien reconnu, auteur de la signalétique de la bibliothèque centrale de Seattle, dont l'architecte est Rem Koolhaas. La conception et la construction de ce bâtiment ont coûté 200 millions de dollars. 
Dès la première affiche scotchée, même s'il n'y en a qu'une: 0 point. Même chose s'il reste des morceaux de scotch laissés après le retrait des anciennes affiches.

\section{Passez à l'action}

Le meilleur moyen d'aborder le problème des affiches provisoires est d'affronter celui, plus profond, qui rend la signalétique nécessaire. Vous pourriez remplacer l'affichette par une signalétique professionnelle, mais cela ne serait que de la poudre aux yeux. S'attaquer radicalement aux causes de cette signalétique permettra non seulement de nettoyer l'environnement visuel, mais améliorera aussi la manière dont les choses fonctionnent.

\subsection{La signalétique réglementaire est simple, conviviale et courtoise}

\section{Pourquoi c'est important}

\section{Difficulté *}

Il faut bien l'avouer, les bibliothécaires peuvent vite devenir désagréables quand ils rédigent des affiches injonctives. Et vous conviendrez que cette signalétique et les messages discourtois qu'elle véhicule n'améliorent guère l'expérience de l'utilisateur...

Si vous avez suivi les choses dans l'ordre, vous avez déjà supprimé la plupart des affiches injonctives de votre bibliothèque, puisqu'elles ne s'appliquent qu'à une frange minoritaire des usagers et sont inefficaces. Si vous devez maintenir l'affichage d'un rappel réglementaire, assurez-vous qu'il ne fasse pas passer les bibliothécaires pour des peaux de vache moralisatrices ou des donneurs de leçons sentencieux.

Utilisez votre audit pour identifier l'ensemble de la signalétique injonctive ou réglementaire qui reste dans votre bibliothèque. 
Testez le ton de vos affiches en les lisant à voix haute: ce que vous ne pourriez pas dire de vive voix à un utilisateur de la bibliothèque n'est pas non plus à écrire sur une affiche. Faites la chasse à toutes les formules négatives. Recherchez une meilleure manière de formuler ces consignes, notez-la et prévoyez une réécriture.

Si l'ensemble de votre signalétique injonctive est simple, conviviale et courtoise: 10 points.

Si la moitié demande une amélioration: 5 points.

Si plus de $50 \%$ nécessitent une remise à plat complète: 0 point.

\section{Passez à l'action et réécrivez la signalétique}

Cela ne devrait pas prendre trop de temps, puisque, en apprenti designer UX appliqué, vous avez déjà supprimé la plupart des affiches injonctives de votre bibliothèque.

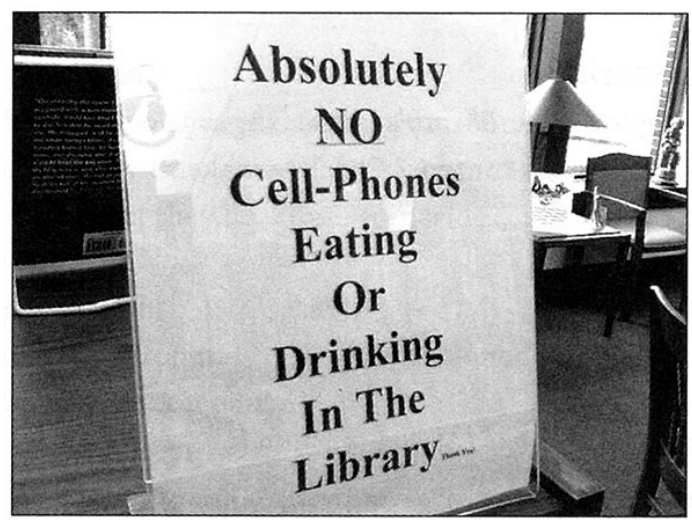

Fig. 6.7 Exemple d'affiche injonctive: Interdiction absolue de téléphoner

Voici un exercice de réécriture pour illustrer comment améliorer une mauvaise signalétique. Une affiche qui ne passe pas le test de ton décrit ci-dessus (figure 6.7) est désobligeante, laide et, sur le fond, tente de réguler les comportements en régulant l'usage 
de la technologie. C'est une perspective complètement erronée et une piètre manière de communiquer. Tout cela compromet la qualité de l'expérience vécue par l'utilisateur.

Une meilleure solution est illustrée par la figure 6.8. Notez qu'elle ne se place pas sous un angle technologique: elle s'adresse au comportement, et ce de manière positive et engageante.

\section{Niceville Public Library}

\section{Polite use of cellphones encouraged!}

Fig. 6.8 Exemple de message bienveillant

\subsection{La carte de bibliothèque}

contient des informations utiles et respecte la charte graphique

\section{Pourquoi c'est important}

\section{Difficulté *}

D'accord, cette étape est un peu la cerise sur le gâteau. Une superbe carte de bibliothèque ne procurera pas, en elle-même, une valeur ajoutée exceptionnelle à l'expérience de l'utilisateur. Malgré tout, ne négligez pas cet élément de votre identité visuelle. La carte est une toute petite part de votre établissement, mais une petite part que les usagers vont très souvent utiliser et rapporter chez eux: assurez-vous qu'elle puisse être jolie et utile. Une carte de bibliothèque, c'est une attention discrète, certes, mais c'est une marque d'attention quand même... 


\section{À vous de jouer}

De deux choses l'une, ou votre carte respecte la charte graphique de votre bibliothèque, ou ce n'est pas le cas. C'est facile à mesurer. Quelles sont les informations type à faire apparaître sur une carte? Assurez-vous qu'elle comprend bien les éléments suivants:

$\odot$ nom de la bibliothèque;

$\odot$ numéro de téléphone;

$\odot$ horaires;

$\odot$ URL du site web;

$\odot$ adresse;

$\odot$ slogan de la bibliothèque;

$\odot$ nom de l'usager;

$\odot$ code-barres.

Accordez-vous de 0 à 5 points selon la qualité de votre carte.

\section{Repensez le design de la carte de bibliothèque}

Utilisez les lignes directrices de votre charte graphique pour concevoir le design de votre carte. Si vous êtes en panne d'inspiration, jetez un œil sur les élégantes cartes professionnelles des grandes entreprises: vous en trouverez de nombreux exemples en ligne.

Si vous concevez une nouvelle carte, vous pouvez tout aussi bien en prévoir plusieurs. Il vous suffit d'utiliser les différentes couleurs de votre charte graphique et de laisser les usagers choisir celle qu'ils préfèrent.

Vous pouvez aussi envisager des cartes de taille différente. Certains usagers préfèrent les versions minimales (sous forme de porte-clés) au lieu du format de type carte de crédit. 


\subsection{Les nouveaux visiteurs repèrent facilement l'organisation générale de la bibliothèque}

\section{Pourquoi c'est important}

\section{Difficulté *}

Bien sûr, faire bonne impression à l'utilisateur dès sa première visite est important. Mais même les usagers de longue date se comportent comme les visiteurs qui découvrent les lieux pour la première fois quand ils viennent à la bibliothèque pour faire quelque chose de nouveau.

Assurez-vous que chaque nouveau visiteur puisse aisément se repérer dans toutes les zones de la bibliothèque. Cela garantira à chacun d'entre eux de passer un moment agréable en se promenant dans le bâtiment.

\section{À vous de jouer}

Trouvez des personnes qui n'ont jamais visité votre bibliothèque. Demandez-leur de se placer dans le hall d'entrée et de repérer des lieux ou des services. Proposez-leur des scénarios type et regardez comment ils s'en sortent. Ces scénarios pourraient être, par exemple:

$\odot$ rendre un document;

$\odot$ trouver où a lieu l'heure du conte;

$\odot$ trouver un livre sur un sujet donné;

๑ retirer un document réservé;

$\odot$ trouver un bibliothécaire pour lui poser une question.

Restez en retrait, suivez-les discrètement et observez-les pendant qu'ils se livrent à l'exercice. Errent-ils à l'aveuglette? 
S'adressent-ils au personnel, ou celui-ci va-t-il au-devant d'eux? Regardent-ils la signalétique? Quels sont leurs points d'arrêt, c'est-à-dire les endroits où ils marquent une pause afin de décider de la direction à prendre? Passent-ils à côté d'affiches qui pourraient les aider?

Cet exercice classique de carte d'itinéraire (voir le chapitre2) peut vous être très utile pour comprendre les différents trajets de personnes qui recherchent la même chose, et le temps que cela leur prend.

Une fois que les volontaires auront atteint leur objectif, posezleur des questions dans la foulée sur leur expérience. Demandezleur ce qu'ils ont trouvé pratique ou, au contraire, déroutant. En associant vos observations et les résultats de la carte d'itinéraire, vous aurez suffisamment de données valables pour déterminer comment ils s'orientent et retrouvent leur chemin dans la bibliothèque.

Si chacun de vos utilisateurs néophytes parvient à remplir son objectif rapidement, avec facilité et sans confusion: 15 points. Vous n'avez rien à faire de plus.

Si à l'inverse la plupart des gens peinent à se repérer dans la bibliothèque: 0 point. $\mathrm{Du}$ travail vous attend.

Mise en garde. Ne trichez pas en demandant à un collègue de la ville d'à côté de se porter volontaire pour ce genre de test. Un bibliothécaire en sait toujours plus que n'importe quel utilisateur type sur la manière d'organiser une bibliothèque. Des amis ou des parents sont en revanche des personnes ressources potentielles, même si dans l'idéal, il vaut mieux faire appel à un des volontaires enregistrés dans votre base de testeurs. 


\section{Passez à l'action et réglez les problèmes d'orientation dans le bâtiment}

Si de nombreux usagers éprouvent des difficultés à identifier un endroit précis de la bibliothèque, étudiez les moyens de rendre ce lieu plus facile à trouver. Mettez en œuvre votre meilleure idée et effectuez quelques tests. Le changement améliore-t-il les choses? Dans le cas contraire, recommencez et faites de nouveaux tests jusqu'à cela soit le cas.

\subsection{Plus de lectures}

Calori C., Vanden-Eynden D., Signage and Wayfinding Design: A Complete Guide to Creating Environmental Graphic Design Systems, $2^{\text {nd }}$ edition, Hoboken, Wiley, 2015.

Chermayeff I., Geismar T., Kenedi A., Haviv S., Identify: Basic Principles of Identity Design in the Iconic Trademarks of Chermayeff \& Geismar, New York, NY: Print Publishing, 2011.

Quelques-unes des réalisations d'un des plus grands cabinets de design américain, auteur notamment du logo de la Library of Congress.

GEL (Global Experience Language), BBC. [En ligne] < http://static. bbci.co.uk/gel/0.5.7/downloads/GEL_web_styleguide.pdf >.

Gibson D., The Wayfinding Handbook: Information Design for Public Places, New York, Princeton Architectural Press, 2009.

Learning Space Toolkit. [En ligne] <http://learningspacetoolkit. org/ >.

Müller-Brockmann J., Grid System in Graphic Design: A Visual Communication Manual for Graphic Designers, Typographers and Three Dimensional Designers, Sulgen, Niggli, 2008. [En ligne] < http://www.sudoc.fr/140017186>.

Un classique absolu en matière de design. 
Williams R., The Non-designer's Design Book: Design and Typographic Principles for the Visual Novice, 4th edition, Berkeley - Peachpit - Londres, Pearson Education, 2015. [En ligne] < http:// www.sudoc.fr/184587573 >. 


\section{Chapitre 7. La présence en ligne}

Il y a de fortes chances pour que les personnes préfèrent consulter le site web de la bibliothèque que de se rendre sur place.

Les efforts que vous investissez dans votre site web sont-ils à la hauteur de l'attention que les utilisateurs lui portent? Peut-être pas. Si vous êtes prêt ou prête à faire le point sur la présence en ligne de votre bibliothèque, ce chapitre est fait pour vous: il vous permettra de comprendre les enjeux de votre site web et de le repenser pour améliorer l'expérience de ceux qui l'utilisent.

\subsection{Trouver des documents et les réserver est facile}

\section{Pourquoi c'est important?}

\section{Difficulté $* * *$}

Avez-vous déjà recherché un livre dans Amazon avant d'en copier/coller la référence dans le catalogue en ligne de votre bibliothèque? Si oui, vous n'êtes sans doute pas le seul. Or, si vous pensez que votre outil de recherche en ligne est poussif, que doivent ressentir vos utilisateurs?

Nombre de catalogues de bibliothèque sont vraiment difficiles à utiliser: c'est un problème majeur. Pourquoi?

Parce que la plupart des gens utilisent le catalogue d'une bibliothèque pour trouver des documents, prolonger des emprunts ou faire des réservations. Avant toute chose, c'est cela qui doit être vraiment facile à faire. C'est une des opérations essentielles effectuées sur votre site! 
Commencez par demander à quelques personnes dans la bibliothèque pourquoi elles vont sur votre site web et écoutez bien leurs réponses. Vous entendrez sans doute beaucoup parler de recherche de documents.

Il est facile d'avoir le formulaire de recherche au milieu de la page d'accueil, voire même au milieu des autres pages du site. Malheureusement, la prise en compte du point de vue de l'utilisateur s'arrête bien trop souvent là. Après avoir lancé leur recherche, les usagers sont renvoyés vers une interface différente et souvent difficile à utiliser.

L'importance de la recherche catalogue sur le site web de la bibliothèque et la difficulté pour l'améliorer sont une véritable épine dans le pied pour tout bibliothécaire qui veut améliorer l'expérience de ses utilisateurs.

\section{À vous de jouer}

Vous pouvez toujours demander aux gens leur opinion sur la recherche, le renouvellement et les réservations de documents. Cependant, un des meilleurs moyens de savoir ce qu'ils vivent réellement quand ils utilisent votre catalogue est de mener des tests d'utilisabilité. Il n'y a qu'en les observant que vous serez capable de savoir où, quand et comment les utilisateurs se perdent en utilisant le catalogue de la bibliothèque.

Concevez un test d'utilisabilité portant sur les principales fonctionnalités de votre catalogue. Essayez un scénario de ce genre (ou toute variation adaptée à votre environnement):

1. Votre fille de 14 ans veut s'inscrire à un atelier de scrapcooking et vous aimeriez en savoir plus sur cette activité. Trouvez trois livres sur le sujet disponibles à la bibliothèque et empruntables.

2. Vous êtes intéressé par le dernier livre de cuisine minceur de Carole Nitsche. Cherchez-le dans le catalogue de la bibliothèque et réservez-le. 
3. Vous avez emprunté trois livres à la bibliothèque et vous ne pouvez pas les rendre à la date prévue. Prolongez vos emprunts.

Suivez la méthode proposée en 7.2 pour conduire le test.

Si les testeurs ont accompli toutes les tâches demandées: 10 points.

S’ils n'ont réussi qu'une partie des missions: 5 points.

S’ils n’ont réussi à effectuer aucune des 3 tâches: 0 point.

\section{Passez à l'action et améliorez l'expérience de recherche}

La plupart des bibliothèques achètent des OPAC (Interfaces de consultation) "clés en main" à leurs fournisseurs de SIGB (Système intégré de gestion des bibliothèques). Certains fournisseurs s'efforcent de proposer de bonnes interfaces et les conçoivent selon une méthodologie UX, orientée vers l'utilisateur, en menant des tests d'utilisabilité. Ce n'est pas le cas de tous. Les bibliothèques ont peu de prise sur le développement des interfaces propriétaires et maîtrisent peu le résultat final. Éditer les feuilles de style et utiliser les solutions de contournement offertes par JavaScript permet quelques améliorations, mais il est souvent difficile d'aller bien loin.

Il faut donc être créatif et renoncer à l'interface de consultation fournie avec le SIGB. Certaines bibliothèques y parviennent en utilisant des applications tierces pour valoriser leurs collections - elles mettent, par exemple, en valeur les nouveautés sur leur page d'accueil sous forme de carrousel de couvertures via une application comme LibraryThing <www.librarything.com > ou Goodreads < www.goodreads.com >, ou bien en récupérant des fichiers de fournisseurs comme Electre ou certains libraires. Si ces outils ne corrigent pas vraiment la piètre qualité de l'utilisabilité des catalogues, ils peuvent aider à améliorer la navigation et la découverte des collections de la bibliothèque. 
Vous pouvez faire des changements plus structurels sur votre catalogue en utilisant un outil de découverte. Parmi les options à votre disposition Blacklight <http://projectblacklight.org/>, BiblioCommons <www.bibliocommons.com >, Elastic Search $<$ https://www.elastic.co/fr/products > et VuFind < http://vufind. org > présentent l'avantage d'être des logiciels libres, utilisables gratuitement, si vous avez un bon informaticien sous la main.

En bibliothèque universitaire, certains outils de découverte comme Summon, Primo ou Ebsco EDS permettent de surcroît d'intégrer dans la recherche l'interrogation des articles en ligne et, moyennant des paramétrages personnalisés, d'avoir des listes de résultats classées par type de document et des outils performants pour affiner la recherche en fonction de critères variés.

Ces interfaces permettent aux bibliothèques de continuer à utiliser le même SIGB pour les activités de back-office et de rendre plus utilisable l'interface publique. Mettre en place ce type d'outil est relativement complexe, mais cela n'implique pas de conduire une réinformatisation complète ni de changer de SIGB, ce qui en fait un bon compromis.

\subsection{Facilitez les opérations essentielles}

\section{Difficulté *}

Vous est-il déjà arrivé, en consultant le site de votre banque, d'être distrait par une liste d'animations dans les succursales de la région, de vous voir proposer, dès la page d'accueil, un calculateur de retraite, des contrats d'assurance-vie ou d'épargne logement?

Si vous n'avez jamais eu ce (dé)plaisir, il y a une bonne raison: votre banque a décidé de simplifier l'expérience de ses clients en ligne. Elle sait bien que la plupart d'entre eux vont sur son site pour y effectuer des opérations précises (consulter ses relevés de 
compte, faire un virement, contacter un conseiller...) et non pour se tenir au courant des dernières nouvelles.

L'autodiagnostic suivant vous aidera à comprendre pourquoi les utilisateurs se connectent au site web de votre bibliothèque et ce qu'ils viennent y faire. Sachant cela, vous pourrez alors leur faciliter les choses.

La raison d'être d'un site web réside dans ce qu'il vous permet de faire. Si vous passez outre cette phase d'analyse, c'est à vos risques et périls.

\section{Pourquoi c'est important}

Chacun peut avoir ses propres motivations pour aller sur votre site web. L'un veut savoir quand il peut venir avec un enfant pour écouter un conte, l'autre a besoin de prolonger un emprunt. Certaines de ces opérations sont plus importantes que d'autres pour eux. Par exemple, chercher un document est encore une des raisons principales de recourir au site web d'une bibliothèque. D’autres opérations, comme contacter par mail la bibliothèque, par exemple, sont très courantes. Prêter attention à ces opérations essentielles et fréquentes est la seule façon de rendre vraiment utile votre site web. De ce fait, vous devez concentrer vos efforts là-dessus. Votre site web permet-il de les accomplir facilement?

Pour déterminer si ces opérations essentielles sont faciles à réaliser, vous avez deux choses à faire:

$\odot$ les identifier;

$\odot$ mener des tests d'utilisabilité sur votre site web.

\section{Identifiez les fonctionnalités essentielles}

Les sites web de bibliothèques proposent souvent de nombreux contenus. Comment pouvez-vous déterminer ce que viennent 
y faire les utilisateurs? C'est simple comme bonjour: en leur posant la question et en les observant.

\section{Entretiens informels}

Les personnes qui viennent à la bibliothèque sont souvent celles qui utilisent le site web: vous n'aurez donc pas de difficultés pour trouver celles à interroger. La seule chose que vous aurez à faire est de sortir de votre bureau et de commencer à leur parler. Vous ne leur prendrez pas beaucoup de temps, puisque vous n'avez qu'une seule question à leur poser: «Pourquoi utilisezvous le site web de la bibliothèque? » Enregistrez leur première réponse et remerciez-les de vous avoir consacré un peu de temps. Vous n'aurez pas besoin de faire beaucoup d'entretiens pour voir émerger plusieurs tendances: assurez-vous d'aller poser la question à des utilisateurs installés dans les différents espaces de la bibliothèque. Vous collecterez ainsi des données d'utilisateurs de différents types. À ce stade, il est recommandé d'interviewer dix à quinze personnes par zone de la bibliothèque.

Après ces entretiens, triez les réponses en fonction de leur fréquence. Les tâches qui se situent en début de classement sont les opérations fondamentales. Votre site devra permettre de les réaliser très facilement.

\section{Statistiques web}

Comparez ce que disent les personnes interrogées avec vos statistiques d'utilisation. Il y a toutes les chances pour que ce qu'elles disent faire sur le site et ce qu'elles y font effectivement soit un peu différent! Comparez les données recueillies et les statistiques: elles révéleront quels sont les contenus très utilisés qu'ils n’ont pas mentionnés. Ajoutez-les à la liste des opérations essentielles. 
Aller plus loin que les fonctionnalités essentielles

Réfléchir aux fonctionnalités essentielles est très utile: aller un peu plus loin et examiner les motivations profondes des utilisateurs peut aussi aider à mieux concevoir un site web.

\section{Tests d'utilisabilité}

Connaître les opérations essentielles effectuées via votre site web est un premier pas important: il ne faut pas s'arrêter là. Vous devez ensuite vous assurer que ces tâches sont faciles à effectuer pour les utilisateurs. Il n'y a qu'une façon de le savoir: faire des tests d'utilisabilité.

Les tests d'utilisabilité sont capitaux: un bibliothécaire n'a pas la même vision du site web qu'un utilisateur. Même s'il est possible que des bibliothécaires fassent preuve de discernement et sachent prendre du recul, ils connaissent généralement trop bien le fonctionnement des bibliothèques - et sont trop immergés dans la construction du site web - pour faire des remarques objectives qui conduisent à des améliorations. Les tests d'utilisabilité permettent d'échapper à ces biais et révèlent la façon dont les utilisateurs novices perçoivent et utilisent le site web de la bibliothèque.

\section{Tests d'utilisabilité : un bref mode d'emploi}

Qu'est-ce qu'un test d'utilisabilité? Un test d'utilisabilité est une technique d'analyse des usages consistant à observer quelqu'un qui est en train d'utiliser un produit. Dans le cas d'un site web, l'observateur regarde une personne utiliser ce site. Donc pour tester celui de votre bibliothèque, vous devez observer de manière réflexive quelqu'un en train d'accomplir - ou en train 
d'essayer d'accomplir - une tâche. Au cours du processus, vous découvrirez où les utilisateurs rencontrent des difficultés sur votre site web et ce que vous pouvez faire pour le rendre plus facile à utiliser. Organisez des tests d'utilisabilité. Conduire des tests d'utilisabilité est une activité simple que vous gagnerez à planifier soigneusement. Une après-midi ou une matinée de tests d'utilisabilité s'organise grosso modo ainsi. Trouvez trois à cinq testeurs. Bonne nouvelle: vous pouvez demander à n'importe qui de faire des tests d'utilisabilité. Les seuls que vous devez éviter sont les professionnels de la bibliothèque et les personnes qui n’ont absolument aucune expérience du Web. Les employés de la bibliothèque en connaissent trop pour être des testeurs objectifs, et quelqu'un qui n'a jamais été sur Internet manquera des compétences nécessaires pour mener le test. Autrement dit, quasiment tout le monde fait l'affaire. Les testeurs peuvent être des utilisateurs de la bibliothèque ou des non-utilisateurs et ils peuvent avoir des expériences variées d'Internet. Vous pouvez solliciter des participants dans la bibliothèque, mettre une annonce sur le site de votre ville ou université, dans la presse locale, ou demander à vos amis. Demandez-leur leurs coordonnées, fixez un créneau d'une heure avec eux, et envoyez-leur un rappel la veille du jour J. Développez des scénarios. Durant vos tests d'utilisabilité, vous demandez aux participants d'effectuer des actions précises en utilisant votre site web. Les mots que vous utiliserez sont importants parce qu'ils influenceront ce que feront les personnes interrogées. Si vous cherchez à savoir comment elles s'en sortent pour «interroger les bases de données de la bibliothèque», votre première réaction sera probablement de demander «Pourriezvous, s'il vous plaît, rechercher un article en utilisant nos bases de données». Ces trois mots «rechercher», «article» et «bases de données» peuvent donner aux intéressés trop d'indices sur ce qu'ils ont à faire. Au lieu de leur transmettre des instructions littérales, vous devez présenter des scénarios fictifs. Voici un 
scénario qui peut fonctionner pour tester la recherche d'informations: «Vous vous entraînez pour votre premier marathon. Vous cherchez un guide pratique sur le régime et la préparation physique à suivre.» $\mathrm{Ou}$ en version niveau Master/Doctorat: "Vous avez entendu un reportage à la radio sur de nouvelles recherches sur le régime alimentaire et les performances en course de fond et vous voulez en savoir plus. Utilisez le site web de la bibliothèque pour trouver des informations précises sur ces nouvelles recherches.» Les scénarios éviteront d'orienter les personnes interrogées vers certaines parties du site et votre test n'en sera que plus réaliste. Pendant les tests, vous devez avoir une liste de scénarios à lire que vous remettrez aussi sur papier aux testeurs, en guise de pense-bête. Rédigez un script. Suivre un script permet de s'assurer de garder la main pendant la conduite du test d'utilisabilité. Après avoir accueilli les testeurs en les mettant à l'aise, ce qui exige de rester assez informel au départ, tout ce que vous aurez à faire sera de lire les scénarios prérédigés et de vous assurer que tous les testeurs ont entendu les mêmes instructions. Votre test y gagnera en rigueur et ses résultats en validité. Le rôle majeur du script est de rassurer et accompagner les testeurs. En effet, ils peuvent être inquiets que vous les jugiez négativement s'ils n'arrivent pas à faire ce que vous leur demandez, ou se sentir gênés d'être observés. Soyez clairs sur l'objectif de la session: c'est le site de la bibliothèque qui est testé, pas eux! Rappelezleur qu'il ne faut pas avoir peur de faire des erreurs parce qu'il n'y a pas de «mauvaise réponse». Encouragez-les à dire tout ce qui leur passe par la tête à propos du site au cours de leur recherche et rappelez-leur qu'ils ne doivent avoir peur de ne vexer personne. On trouve de bons exemples de scripts en ligne, y compris en français. Préparez les lieux. La préparation de votre salle n'est pas compliquée. Vous devez juste vous assurer d'avoir un lieu au calme, sans distraction, pour pouvoir conduire les tests dans de bonnes conditions. Un bureau, une salle de travail en groupe, ou 
même un coin tranquille de la bibliothèque conviendront. Évitez de conduire des tests avec des ordinateurs portables, car certaines personnes n’y sont pas habituées. Utilisez des ordinateurs standard avec clavier et souris. Si vous avez des ordinateurs équipés de Microsoft Windows ou d'Apple OS X, allumez les deux et donnez aux testeurs la possibilité de choisir leur système d'exploitation. Si vous voulez que d'autres employés de la bibliothèque observent les tests, vous devrez aussi installer un micro et un logiciel de partage d'écran. Que devez-vous enregistrer? Quand vous menez le test, notez si les testeurs ont fait tout ou partie de ce qui leur avait été demandé. Vous pouvez aussi prendre des notes quand un testeur se trouve bloqué, en consignant ses propositions ou vos idées sur la meilleure façon de pallier les problèmes rencontrés et tout ce que les testeurs peuvent dire d'intéressant pendant qu'ils utilisent votre site. Vous pouvez choisir d'enregistrer le test grâce à un logiciel de capture d'écran comme ScreenFlow < www.telestream.net/screenflow/overview. htm>, mais ce n'est vraiment pas nécessaire. Si vous le faites, revoir la vidéo doublera le temps consacré au test sans vraie valeur ajoutée. Vous avez tout à gagner à utiliser ce temps pour faire un nouveau test plutôt que pour revoir le même. Tout au plus, si vous menez un test d'utilisabilité pour la première fois, l'enregistrement pourra-t-il vous aider à prendre confiance en vous, à vérifier que vous n'avez pas laissé passer d’informations importantes et à améliorer votre manière de faire pour la fois suivante. Qui observe le test d'utilisabilité? Si le site web de la bibliothèque n'est pas un cheval de bataille politique et que vous voulez juste comprendre comment l'améliorer, vous n'avez pas besoin d'y associer qui que ce soit. Vous pouvez observer, comprendre, faire les changements vous-même puis recommencer. Les tests d'utilisabilité peuvent aussi permettre d'aller plus loin que la seule ergonomie du site web. Ils peuvent vous aider à sensibiliser les décideurs et à infléchir leur point de vue. Si la direction de la 
bibliothèque constate en direct les difficultés rencontrées par les utilisateurs du site web, cela peut vous donner des arguments concrets pour augmenter les ressources humaines et matérielles dédiées aux outils en ligne. Cela peut aussi vous aider à dépasser les discussions stériles entre collègues, fondées sur des idées toutes faites et des préférences personnelles, pour enfin discuter - sur pièces - des comportements réels des usagers. Pensez à inviter les bonnes personnes pour assister aux tests si vous estimez que cela peut être un levier de changement. Si vous voulez que d'autres personnes assistent aux tests, ne les installez pas dans la même salle que vous. Cela ne sera confortable ni pour les spectateurs ni pour les testeurs qui peuvent être intimidés. Il est préférable d'utiliser un logiciel de partage d'écran comme join.me $<$ https://join.me > afin de projeter le test dans une autre pièce ou, si vous n'avez pas le choix, d’organiser en différé une séance de visionnage des tests. Rendez compte des résultats. Si vos collègues regardent les tests en direct, ne différez pas la discussion et programmez une réunion ou un déjeuner dans la foulée afin que les détails soient encore frais dans leur esprit. Les tests d'utilisabilité doivent vous permettre de dégager des priorités d'action et un consensus. Certaines mesures très simples permettent parfois d'améliorer de manière significative l'utilisabilité des sites, sans nécessiter de coûteux développements. Si vous faites des tests seulement pour faire un diagnostic, sans action corrective programmée dans la foulée, vous pouvez écrire la synthèse de ce que vous avez appris et la transmettre aux personnes concernées. Testez tôt et réitérez le processus. Ne vous contentez pas d'une seule séance de tests d'utilisabilité. Idéalement, vous devriez entrer dans une routine de: tests; résolution de problèmes; nouveaux tests. C'est un processus continu et itératif d'améliorations, qui est bénéfique à la fois pour le moral de l'équipe et pour les usagers de la bibliothèque. 


\section{À vous de jouer}

Quel était le taux de réussite de votre test? Est-ce que tous les testeurs sont parvenus à faire l'intégralité de ce que vous leur demandiez? Est-ce que la plupart des testeurs ont eu des difficultés avec certaines tâches? Le calcul du CUTI à l'issue d'un test d'utilisabilité sera forcément un peu subjectif.

Si vous êtes globalement satisfait de la façon dont votre site fonctionne: 20 points.

S’il y a beaucoup de choses à améliorer: 10 points ou moins.

Conseil. Si vous concevez un nouveau site web ou si vous faites une refonte de votre site, vous n'avez pas à attendre que les changements soient effectifs pour mener un test d'utilisabilité. Tester des prototypes est un moyen remarquablement efficace de gagner du temps et de gommer les défauts avant de mettre le site en production. Cela vous évitera de décevoir les utilisateurs. Le test sur prototypes est une bonne méthode, quel que soit votre processus de conception et de développement. Si vous avez des maquettes web, utilisez-les pour les tests. Mais n'oubliez pas que vous pouvez aussi tester des maquettes faites avec Photoshop ou même des esquisses sur papier. Pour en savoir plus sur cette forme de tests d'utilisabilité, voir Carolyn Snyder, Paper Prototyping (Morgan Kaufmann, 2003).

\subsection{La richesse en contenus de votre site est adaptée au temps que vous pouvez y consacrer}

\section{Pourquoi c'est important}

\section{Difficulté*}

Créer une nouvelle page sur un site web est facile et ne prend que quelques minutes. Remplir cette page avec du contenu pertinent 
et bien rédigé, l'illustrer d'une bonne image et la tenir à jour demande un effort autrement soutenu! Bien des bibliothèques ont cédé à la tentation de créer des pages et des pages pour leur site web. Chaque service réclamant d'être présent sur le site web, on comprend pourquoi de nouvelles pages ou des sous-sites sont sans cesse créés. Mais ces passions d'un jour débouchent rarement sur une histoire durable. On les laisse bientôt prendre la poussière tandis qu'une nouvelle activité plus séduisante attire l'attention. Résultat: un site mal ficelé, rempli de contenus sans intérêt. Plutôt que de multiplier les histoires sans lendemain avec des contenus dénués de sens, lancez-vous plutôt dans une relation durable avec des contenus qui en valent la peine.

Avoir un petit site web offre bien des avantages. Plus votre site sera petit, plus vous serez attentif à chaque contenu. Ne dispersez pas vos efforts: réduire le nombre de pages que vous aurez à maintenir libérera du temps et vous permettra de vous concentrer sur les pages importantes.

Plus vous avez de contenus, plus vous devrez prendre de décisions de mise en forme. Chaque nouvelle décision est une occasion de faire une erreur. Ces contenus exigent une architecture, un nommage, une navigation, un graphisme visuel de plus en plus sophistiqués. Il est impossible de faire toutes ces choses correctement et l'utilisateur risque rapidement d'avoir l'impression de chercher une aiguille dans une meule de foin. Plus la meule de foin sera petite, plus vite il y trouvera ce qu'il cherche.

En réalité, décider dès le départ que votre site doit être restreint est une décision raisonnable. Il est toujours préférable de centrer son site sur les opérations essentielles pour les visiteurs et de s'en tenir à cela.

\section{À vous de jouer}

La taille de votre site dépendra de la taille de votre équipe web et de l'état de votre site actuel. Le diagnostic 7.4 vous proposera 
de faire un audit de contenu. Vous pouvez l'utiliser pour évaluer la qualité de votre site dans son ensemble. Combien de pages ne sont-elles consultées que quelques secondes?

Si plus du quart de vos pages web nécessitent une sérieuse amélioration, c'est que votre équipe web se disperse. Vous pouvez y remédier, soit en engageant plus de monde, soit en réduisant le nombre de pages de votre site. Au vu de la situation budgétaire de la plupart des bibliothèques, la deuxième option est probablement plus réaliste. Elle est peut-être même préférable dans l'absolu. Souvenez-vous: sur le Web, «less is more», moins c'est mieux. Ou tout simplement: "less is less", moins c'est moins, et c'est bien comme ça. En fait, c'est même parfait.

Votre site a juste la taille qu'il faut, chaque page est fréquentée et à jour? 10 points.

Il est énorme, s'éparpille et il n'y a pas assez de monde pour s'en occuper? 0 point.

\section{Passez à l'action et élaguez votre site}

Qu'allez-vous garder? Qu'allez-vous élaguer? Ce sont des questions importantes. Alléger votre site ne doit pas être un casse-tête. Pensez à vos contenus comme à un compromis équilibré entre:

$\odot$ ce que veulent faire vos utilisateurs sur le site;

$\odot$ ce que la bibliothèque veut ou doit absolument y inclure;

$\odot$ ce que l'organisation et les ressources de la bibliothèque permettront de gérer sur le long terme.

\section{Les besoins des utilisateurs}

Ce sont les opérations essentielles que les utilisateurs font sur le site. Allez voir le point de repère 7.2 pour les identifier. Gardez toutes les fonctionnalités essentielles. 


\section{Les besoins de la bibliothèque}

Dans la plupart des bibliothèques, chaque équipe a des demandes spécifiques. Si rien n'est mis en place pour évaluer ces demandes et faire le tri, il devient malaisé d'y faire face de façon constructive; les collègues peuvent se sentir frustrés et le webmaster débordé, ce qui n'est bon pour personne. La plupart du temps, les contenus finissent par être ajoutés et les sites web des bibliothèques accumulent les contenus accessoires.

Pour éviter cela, vous pouvez mettre en place une stratégie de gestion des contenus compréhensible par tous. Le fait de poser des règles claires sur le type de contenu que le site web recueillera permet de stimuler chacun dans sa contribution à l'objectif principal du site plutôt que de chercher à imposer à tout prix son point de vue ou des détails mineurs concernant son seul service.

\section{La capacité d'organisation}

Évaluer la capacité de travail de votre équipe web vous aidera à déterminer la quantité de contenus à supprimer. Peut-être que votre bibliothèque a une capacité suffisante pour maintenir correctement cinquante pages ou seulement vingt-cinq. Mieux vaut pécher par excès de prudence. Si, après avoir élagué le site, votre équipe web a la situation parfaitement sous contrôle, voire tue le temps en faisant de la veille sur YouTube, il sera bien temps, alors, de penser à ajouter quelques pages.

\section{Imaginer une version mobile}

Une autre manière de réfléchir à ce qui est le plus important dans votre site web est d'imaginer une version mobile... ou même de la mettre en place dans la foulée. Compte tenu de la taille limitée des écrans de smartphones, quelle partie de votre site est vraiment essentielle? Cette liste sera probablement très proche de la liste des fonctionnalités essentielles. 


\section{Les statistiques}

Les statistiques de votre site web devraient aussi vous aider, mais elles peuvent être délicates à interpréter. Le nombre de pages vues ne donne pas d'informations sur ce qui motive les visites. Certaines pages peuvent être ignorées, soit que leur contenu n'intéresse pas les utilisateurs, soit qu'elles sont juste difficiles à trouver ou à utiliser. Un manque de visites ne signifie pas forcément que la page n'est pas utile, mais il faut en tenir compte et en comprendre les raisons. Utilisez les statistiques avec discernement. Une fois que vous avez déterminé ce qu'il est vraiment important de conserver, considérez le reste comme éventuellement intéressant mais pas nécessaire.

\section{En cas de doute, élaguez!}

Si vous n'êtes pas sûr de l'utilité de certains contenus, enlevez-les. S'il s'avère qu'ils étaient importants et que les internautes en ont besoin, ils se feront entendre. Vous pourrez toujours remettre du contenu, mais il y a fort à parier que vous n'aurez pas à le faire.

\subsection{Le contenu web est attrayant}

\section{Pourquoi c'est important}

\section{Difficulté **}

Pour commencer, une évidence: les utilisateurs visitent votre site web pour y trouver quelque chose de précis. Son contenu est sa raison d'être. Votre bibliothèque doit donc prendre très au sérieux ce qu'elle met et dit sur son site. Non seulement le contenu doit être soigné dans sa forme, adapté aux usages du Web et mis en forme pour le Web, mais il doit aussi être pertinent sur le fond et répondre aux besoins de vos utilisateurs.

Amanda Etches et Aaron Schmidt expliquent avoir travaillé sur les sites web de bibliothèques durant des années et disent: 
«Si nous nous réjouissons de voir une amélioration générale dans la façon dont les bibliothèques planifient et développent leur présence sur le Web, le contenu des sites continue de nous consterner. De nombreuses bibliothèques ont compris que la navigation, la recherche, le fait de trouver ce qu'on cherche sont des points importants, mais ces mêmes bibliothèques considèrent les plannings de publication, le développement et la gestion du site après coup.»

La structure, l'architecture et le design d'un site web sont cruciaux. Les mots et les images présents sur chaque page sont tout aussi importants. Un site web parfaitement conçu et intuitif dont le contenu ne correspond pas aux besoins des utilisateurs est complètement vain. Pour dire les choses autrement: liriez-vous, en tant que bibliothécaire, un magazine entièrement dédié aux tronçonneuses? Probablement pas. Même si le magazine était bien écrit et rempli de belles photographies, un tel sujet ne retiendrait pas longtemps votre intérêt. Belles images et clarté des textes ne suffisent pas à rendre un sujet intéressant, s'il ne fait pas partie de vos centres d'intérêt. Si vous étiez une scierie ou un bûcheron, en revanche, un tel magazine reprendrait tout son intérêt. Pour votre site web, c'est pareil: peu importe qu'il soit joli et bien écrit si son contenu n’intéresse pas la communauté que vous desservez.

\section{À vous de jouer}

L'idéal serait d'avoir une méthode toute faite pour évaluer le contenu de votre site. Malheureusement, il ne s'agit pas juste de voir s'il y a bien des photos de jolis chatons et la météo locale en page d'accueil. C'est un peu plus compliqué parce que les besoins de vos utilisateurs sont variés. Heureusement pour vous, il existe quand même une méthode assez simple pour vérifier si le contenu est utile. 


\section{L'audit de contenu}

L'objectif de l'audit de contenu est d'inventorier et d'évaluer le contenu de votre site de manière critique. Ce processus vous aidera à identifier les contenus à améliorer, à supprimer et à ajouter.

Un audit de contenu consiste à passer en revue chaque élément de votre site: chaque page mais aussi chaque PDF, chaque fichier audio et vidéo, bref, tout ce que vous y avez mis. Cela peut être une tâche ingrate. Si votre site est très gros, demandez de l'aide à tous ceux, qui, dans la bibliothèque, contribuent au site web. Un audit de contenu est une collection de métadonnées sur votre site. Si vous travaillez avec des collègues qui aiment cataloguer, ils aimeront les audits de contenu.

\section{Organisez un audit}

L'audit sera mené à l'aide d'une feuille de tableur comme celui de la figure 7.1. Les premières choses que vous allez enregistrer sont descriptives:

$\odot$ numéro de page;

$\odot$ URL;

$\odot$ titre ou nom de la page;

$\odot$ type de fichier.

\begin{tabular}{|c|c|c|c|}
\hline$\because:$ & A & 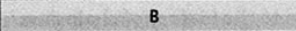 & 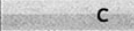 \\
\hline 1 & PAGE ID & URL & NAME \\
\hline 2 & 1.0 & http:uxlibrary.org & home page \\
\hline 3 & 1.1 & http:uxlibrary.org/research & Research \\
\hline 4 & 1.2 & http:uxlibrary.org/reading & Reading \\
\hline 5 & 1.3 & http:uxlibrary.org/cafe & Library Cafe \\
\hline 6 & 1.2 .1 & http:uxlibrary.org/reading/comics & Comic Books \\
\hline 7 & 1.2 .2 & http:uxlibrary.org/reading/classics & Classics \\
\hline
\end{tabular}

Fig. 7.1 Audit de contenu

Le bénéfice de l'exercice réside dans la collecte d'informations qualitatives. Pour évaluer la pertinence de votre contenu, vous devrez vous intéresser à trois aspects principaux. 
Utilité pour les membres de votre communauté

Adoptez le point de vue de vos utilisateurs et demandez-vous: "À quel besoin ce contenu répond-il? À qui manquerait-il s'il était supprimé? » Faites preuve d'honnêteté intellectuelle en répondant à cette question. Ne gonflez pas votre score juste parce que vous pensez que ce contenu pourrait peut-être, un jour, avec un peu de chance, finir par servir à quelqu'un. Mobilisez tout ce que vous savez sur les fonctions essentielles de votre site (voir 7.2) et des centres d'intérêt de vos utilisateurs pour mener à bien cet examen.

\section{Utilité pour la bibliothèque}

Ce contenu est-il assez important pour la bibliothèque pour être en ligne? Sa suppression aurait-elle des conséquences sérieuses?

\section{Usage}

Quel est le trafic sur cette page? Créez une échelle de notes pour chaque élément de contenu et respectez-la. Par exemple l'échelle pour «utile aux utilisateurs» et «utile à la bibliothèque» pourrait être:

$\odot 0$ - inutile;

$\odot 1$ - assez utile;

$\odot 2$ - essentiel.

Une échelle de CUTI pour «l'usage» pourrait être:

$\odot 0-<1000$ visites par mois;

$\odot 1$ - 1001 à 5000 visites par mois;

$\odot 2$ - > 5001 visites par mois.

Il ne vous aura pas échappé que faire un audit de contenu n'est ni rapide ni facile. Cela nécessite du temps et le travail d'un grand nombre de gens. Plus votre site est riche, plus cela nécessitera de temps et de monde! L'effort en vaut pourtant la peine. 


\section{Pour calculer ce CUTI:}

$\odot$ si plus de $75 \%$ des contenus de votre site ont la note maximale sur ces trois aspects: utile aux membres, utile à la bibliothèque et usage: 10 points;

$\odot 50 \%$ des contenus du site ont la note maximale: 5 points;

$\odot$ moins de $50 \%$ : 0 point.

\section{Passez à l'analyse de votre contenu}

Une fois ces critères complétés, vous pouvez trier les colonnes de votre tableau pour en tirer quantité d'enseignements.

Quel pourcentage de votre contenu a reçu 0 ou 1 point pour « utile aux utilisateurs»? Quel est le contenu le plus populaire de votre site? Quels contenus sont ignorés? Si vous découvrez du contenu qui n'est pas très utile et qui reçoit peu de trafic, supprimez-le. Il ne manquera à personne! Si vous découvrez du contenu vraiment utile qui reçoit peu de trafic, trouvez un moyen de le rendre plus facilement accessible.

Grâce à cet audit, vous constaterez qu'en mettant en lumière les faiblesses de votre site, vous découvrez du même coup comment l'améliorer. Comme la plupart des analyses d'usages, cette première exploration de votre contenu ne doit pas être la dernière. Il faut mener ce type d'évaluation de manière régulière, pour maintenir un site utile et utilisable. Par exemple, quand un de vos contributeurs met à jour une page ou ajoute un nouveau contenu, vous devez l'évaluer de la même manière que le reste du site. C'est un bon moyen de vous prémunir contre le risque, apparemment inévitable, d'explosion des contenus qui trouble le sommeil de bien des webmasters.

Des contenus bien pensés. Avant de rédiger un gros bouquet de contenus pour votre site web, relisez le conseil de la 
section 7.3: vos utilisateurs s'accommoderont mieux d'une sélection de contenus en petit nombre calibrés en fonction de leurs besoins que d'une grosse quantité d'informations peu pertinentes.

\section{Tirez le maximum de votre audit de contenu}

Pour faire un tour complet de la question, vous pouvez aussi évaluer d'autres aspects des contenus de votre site web. Voici quelques suggestions:

$\odot$ rédigé pour le Web: ce contenu est-il rédigé de manière concise, facile à lire et plaisante?

$\odot$ à jour: quand a eu lieu la dernière mise à jour du contenu?

$\odot$ exact: l'information fournie est-elle exacte?

$\odot$ utile aux bibliothécaires: les informations peuvent-elles servir aussi aux bibliothécaires?

Vous pouvez utiliser ces informations supplémentaires pour trier de manière utile votre grand tableau d'audit des contenus. En particulier, assurez-vous scrupuleusement de la qualité de la rédaction et de l'exactitude des contenus les plus consultés.

\subsection{Rédigez pour le Web}

\section{Pourquoi c'est important}

\section{Difficulté*}

Un site web relève du domaine de l'édition. En bon éditeur, vous devez faire très attention à la manière dont les choses sont rédigées. C'est tout naturel: après tout, les gens viennent sur votre site pour utiliser ses contenus. Puisque c'est important, cela vaut la peine de passer du temps à travailler en profondeur l'écriture des pages web. 
Qu'a donc de si spécial l'écriture pour le Web? Tout simplement, de prendre en compte les habitudes de lecture en ligne des internautes.

Ceux-ci ont souvent quelque chose de précis à faire quand ils utilisent le Web. Ils recherchent de l'information ou à effectuer une démarche en particulier. Et ils veulent aller vite. De ce fait, au lieu de lire chaque mot de la page, ils ont tendance à lire très rapidement et à se contenter de la survoler. Il faut donc adapter votre site web à ce type de lecture.

Vous n'avez pas besoin d'attendre la refonte de votre site pour rédiger du contenu adapté au Web. Réécrire des pages existantes est une façon efficace et peu coûteuse d'améliorer sensiblement votre site.

Rédiger pour le Web n’est ni naturel ni automatique.

Si vous ne l'avez jamais fait: 0 point.

Si vous avez déjà réécrit tout votre site pour le Web (ou quand vous l'aurez fait): 10 points.

\section{Passez à l'action et réécrivez vos pages}

Commencez d'abord par modifier vos pages les plus consultées et ne vous arrêtez pas avant d'avoir révisé la rédaction de toutes vos pages. Si une page ne vaut pas la peine d'être réécrite, elle n'a sans doute rien à faire sur votre site. Plutôt que de réécrire chaque page en partant de zéro, éditez votre texte plusieurs fois en l'améliorant à chaque passage. Par exemple, commencez par supprimer des mots, puis réorganisez le contenu en forme de pyramide inversée, puis réécrivez les liens si nécessaire et ainsi de suite.

Utilisez le guide qui suit pour vous aider dans le processus de réécriture. 


\section{Supprimez des mots}

Moins vous utilisez de mots, moins les lecteurs auront à réfléchir. Pour les débutants, ne craignez pas d'utiliser des segments de phrases qui ne contiennent qu'une information à la fois. Par exemple:

Incorrect: Placer une réservation sur un document

Correct: Réserver un document

Évitez les images passe-partout

$\mathrm{Au}$ mieux, les photos trouvées sur Flickr, Corbis ou Fotolia n'ajoutent pas de valeur à votre page web, au pire (et c'est souvent le pire) ce ne sont que des distractions qui donnent à la page l'air brouillon. Si vous utilisez des photographies, assurezvous qu'elles soient de très bonne qualité et en lien avec votre bibliothèque.

\section{Utilisez la pyramide inversée}

Après avoir réduit le nombre d'éléments sur la page, vous pouvez réorganiser ceux qui restent pour les rendre plus faciles à lire.

Regroupez les informations essentielles en haut de la page. C'est là que les internautes sont le plus enclins à regarder. Placer en haut de page une information importante augmente ses chances d'être lue. Mettre un texte d'introduction ou un mot de bienvenue en haut de la page est une perte de temps. Les informations secondaires doivent être reportées en bas de page.

\section{Faites attention aux titres}

Concevez vos titres comme des moyens d'étiqueter les contenus. Ils font gagner du temps au lecteur qui glane et lit de l'information en diagonale. Si un titre lui donne une information directement utile, il continuera de lire. Sinon, il continuera à parcourir la page, de titre en titre, jusqu'à trouver exactement ce qu'il 
cherche. Pour être plus efficaces, les titres doivent être visuellement distincts de l'information qu'ils décrivent. Une bonne façon de procéder est de les rendre plus grands, plus gros ou d'utiliser une couleur différente. Ne laissez pas vos titres au milieu d'une page sans être reliés à un contenu précis. C'est ce qui arrive lorsque l'espace entre un titre et le paragraphe qui le suit est trop important. Dans la figure 7.2, comparez la première colonne (mauvaise façon de faire) avec la seconde (bonne façon de faire).

\begin{tabular}{|c|c|}
\hline $\begin{array}{l}\text { content content content content } \\
\text { content content content content } \\
\text { content content content content } \\
\text { content content content content } \\
\text { content content content content } \\
\text { content content content content } \\
\text { content content content content } \\
\text { content content content content } \\
\text { HEADER } \\
\text { content content content content } \\
\text { content content content content } \\
\text { content content content content } \\
\text { content content content content } \\
\text { content content content content } \\
\text { content content content content } \\
\text { content content content content } \\
\text { content content content content } \\
\text { HEADER }\end{array}$ & $\begin{array}{l}\text { HEADER } \\
\text { content content content content } \\
\text { content content content content } \\
\text { content content content content } \\
\text { content content content content } \\
\text { content content content content } \\
\text { content content content content } \\
\text { content content content content } \\
\text { content content content content } \\
\text { HEADER } \\
\text { content content content content } \\
\text { content content content content } \\
\text { content content content content } \\
\text { content content content content } \\
\text { content content content content } \\
\text { content content content content } \\
\text { content content content content } \\
\text { content content content content } \\
\text { HEADER } \\
\text { content content content content } \\
\text { content content content content } \\
\text { content content content content }\end{array}$ \\
\hline
\end{tabular}

Fig. 7.2 Titres et contenu

\section{Rédigez des liens explicites}

Les liens doivent être descriptifs, privilégier la forme active pour les verbes, et n'être ni trop longs ni trop courts. Voici quelques exemples de formules à rechercher ou à bannir:

$\odot$ à éviter: Pour accéder à votre compte, cliquer ici;

$\odot$ acceptable: Votre compte;

$\odot$ la meilleure solution: Accéder à votre compte.

Utilisez les listes à puces et passez à la ligne

Comme les titres, les listes à puces aident les utilisateurs à parcourir rapidement la page des yeux. Si vous avez une liste dans un 
paragraphe, pensez à séparer les items par des puces. Comparez ce paragraphe:

Pour obtenir une carte de bibliothèque, vous devez vous munir d'un permis de conduire, d'une carte d'identité ou un justificatif de domicile.

Avec cette version:

Pour obtenir une carte de bibliothèque, vous devez présenter:

$\odot$ permis de conduire;

$\odot$ carte d'identité;

$\odot$ justificatif de domicile.

En espérant qu'il ne faut pas toutes ces pièces pour obtenir une carte de votre bibliothèque! Sinon, reportez-vous au chapitre 5.

\section{Adoptez le ton de la conversation}

Quand les utilisateurs de la bibliothèque cherchent un service ou une information sur le site, c'est comme s'ils entamaient une forme de dialogue. Ils doivent se dire quelque chose comme:

«À quelle heure ouvre la bibliothèque le samedi?»

«J'ai besoin de rendre ce DVD après la date prévue, comment faire?»

"Quand est-ce que je peux amener mon fils écouter un conte?»

Si vous voulez rendre votre contenu facile compréhensible, vous pouvez le présenter de cette manière. Anticiper les questions que les gens se posent et les utiliser comme titres est une manière très efficace de rédiger. Quand ils parcourent la page des yeux, ils reconnaissent une question similaire à la leur et comprennent qu'il faut lire la suite. Regardez les figures 7.3 et 7.4 qui présentent une même page avant et après sa réécriture, selon cette technique. 


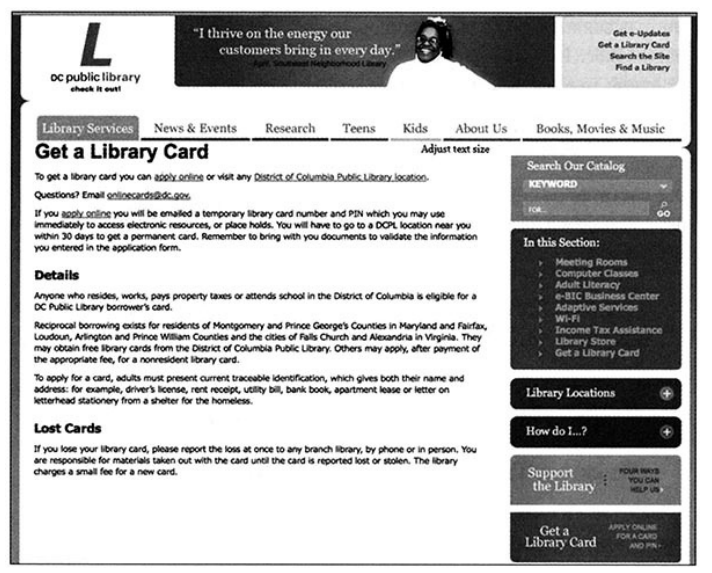

Fig. 7.3 Avant révision

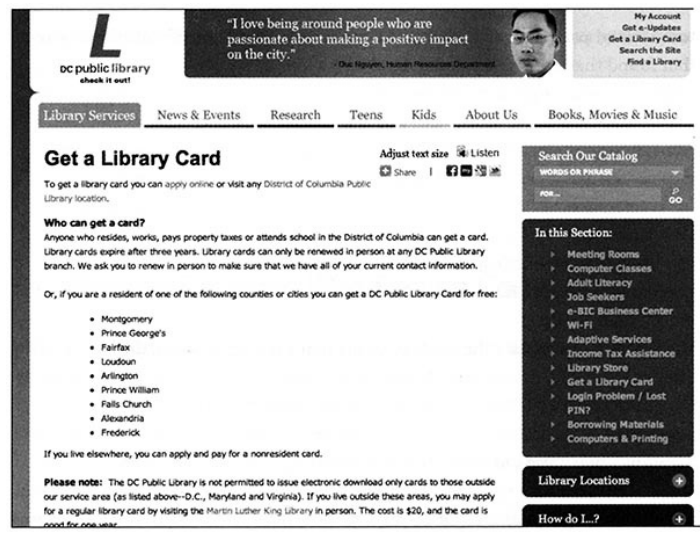

Fig. 7.4 Après révision

Une façon infaillible de garder le ton de la conversation sur le Web est d'éviter la voie passive et de lui préférer, le plus possible, la voie active. Il est facile de tomber dans le piège de la voie passive, car elle donne l'impression d'être plus institutionnelle et plus professionnelle. En réalité, les phrases à la voie passive ont surtout l'air froides et guindées.

Des copies de la lettre d'information de la bibliothèque peuvent être trouvées sur la page «À propos»: à éviter. 
Vous trouverez notre lettre d'information sur la page «À propos»: une meilleure formulation.

\section{Évitez le jargon}

Le jargon induit les utilisateurs en erreur et leur donne l'impression d'être stupides. Il s'insinue dans le site dès qu'on oublie que les utilisateurs de la bibliothèque ne sont pas bibliothécaires. En supprimant le jargon de votre site, vous sortirez de cette vision «bibliocentrée». Le langage naturel aidera vos utilisateurs à vous comprendre et à se servir de votre site.

Pour améliorer le langage de votre site web, dans un premier temps, allez voir John Kupersmith's Library Terms That Users Understand <http://escholarship.org/uc/item/3qq499w7>. Kupersmith rapporte en détail des tests d'utilisabilité qui prouvent que les usagers ne comprennent pas les termes suivants:

$\odot$ base de données;

$\odot$ catalogue ;

$\odot$ revues électroniques;

$\odot$ ouvrage de référence;

$\odot$ ressources;

$\odot$ les noms de marques (i.e. EBSC0);

$\odot$ les acronymes (i.e. SIGB).

Le rapport suggère des mots que les usagers comprennent:

$\odot$ trouver des livres;

$\odot$ services;

$\odot$ mon compte lecteur.

Le rapport de Kupersmith est un bon début, mais ne vous en servez pas pour définir les mots que vous utiliserez vous-même. 
Vous obtiendrez de bien meilleurs résultats en faisant quelques tests auprès de vos utilisateurs.

\section{Testez vos intitulés}

Vous pouvez le faire de manière formelle ou passer quelques après-midi dans les salles de lecture et demander à quelques utilisateurs de consacrer un peu de leur temps. Dans les deux cas, l'idée est de leur montrer les intitulés de vos principales rubriques, un à un, et de leur demander ce qu'ils imaginent trouver dans cette catégorie. Rassurez-les sur le fait qu'il n'y a pas de bonne ou de mauvaise réponse et que vous êtes juste intéressé par leur opinion. Notez leurs réponses et comparez leurs suppositions avec le contenu réel des rubriques.

\section{Testez le tri de cartes}

Le tri de cartes est la démarche inverse. Au lieu de commencer par les intitulés pour amener les utilisateurs à parler du contenu, proposez-leur des descriptions de contenus sur des fiches cartonnées, et demandez-leur de les regrouper, puis de trouver un intitulé parlant pour chaque groupe. Référez-vous au chapitre 2, section 2.2 pour une brève introduction au tri de cartes.

Cet exercice teste votre arborescence et vous donne quelques idées sur le type de mots et d'intitulés qui ont du sens pour les membres de votre communauté.

Une fois que vous aurez testé toute l'arborescence et fait les changements nécessaires, il vous faudra refaire l'exercice régulièrement. Pour vous assurer que les intitulés de votre site restent centrés sur l'utilisateur, conduisez des petits tests dès que vous ajoutez quelque chose. Si vous parvenez à les systématiser, la prochaine fois que vous devrez analyser l'intégralité de votre arborescence, cela se fera en un clin d'œil. 


\subsection{Respectez les conventions de design du Web}

\section{Pourquoi c'est important}

\section{Difficulté *}

Quand vous vous arrêtez à un feu de signalisation, quelle couleur attendez-vous pour démarrer en toute sécurité: vert ou rouge? C'est une convention employée dans votre ville, dans les villes avoisinantes, dans tout le pays et en réalité presque partout. Si certaines villes avaient choisi d'autres couleurs pour leurs feux, ce serait le chaos. C'est pourquoi tout le monde s'est accordé pour standardiser les couleurs des feux de signalisation pour tous les moyens de transport.

De même, il y a des conventions pour le Web qui ont fait leurs preuves et que les internautes respectent, qu'ils en aient conscience ou non. Utiliser ces conventions fera du site web de votre bibliothèque un environnement familier pour votre utilisateur, qui n'offre pas de mauvaise surprise et réagit comme on peut s'y attendre. Cela augmentera l'utilisabilité de votre site et sa fiabilité.

\section{Faites votre autodiagnostic}

Vérifiez que votre site web utilise les conventions suivantes. Si ce n'est pas le cas, le mettre aux normes du Web est une manière relativement rapide et simple d'améliorer votre site.

\section{Le logo du site}

Le nom de votre bibliothèque ou son logo doit apparaître en haut à gauche du navigateur sur toutes les pages du site. Texte ou image, le logo doit permettre de revenir à la page d'accueil. 
Cela fonctionne comme une astuce de navigation infaillible pour les utilisateurs. S'ils se perdent en naviguant sur votre site, ils s'attendent à pouvoir revenir à la page d'accueil en cliquant sur le logo en haut à gauche.

\section{Les liens}

Faites des liens hypertexte visuellement distincts. Pour les liens hypertextes, la meilleure façon de faire est de les afficher dans une couleur différente et de les souligner. La convention la plus classique, un peu démodée, est de les mettre en bleu (les premiers navigateurs web affichaient les liens ainsi par défaut, les internautes s'y sont habitués). D’autres couleurs font aussi bien l'affaire: assurez-vous juste qu'elles soient différentes du reste $\mathrm{du}$ texte et contrastent suffisamment avec la couleur du fond. Si vous décidez de choisir du bleu, souvenez-vous qu'aucun texte sur le site, hormis les liens, ne doit être bleu. Il n'y a pas moyen que vos liens cliquables ressortent si d'autres textes, non cliquables, sont aussi en bleu! N'oubliez pas non plus d'attribuer une couleur différente aux liens déjà visités. Par la suite, cela aidera vos visiteurs à s'orienter. Dans l'idéal, la couleur de vos liens visités sera une version plus claire de la couleur que vous avez choisie pour les liens non visités.

\section{Le fil d'Ariane}

Le menu principal de votre site web n'est pas la seule façon de s'orienter sur votre site. Le fil d'Ariane est un autre outil de navigation qui apparaît souvent en haut de la zone de contenu principal d'une page. Les fils d'Ariane les plus courants précisent l'emplacement de la page dans l'arborescence du site.

Si votre site a une architecture complexe, assurez-vous de proposer un fil d'Ariane. Si votre site n'a pas une arborescence très profonde et comporte seulement un ou deux niveaux, vous pouvez probablement vous en passer. 


\section{Contacts et informations pratiques}

Beaucoup d'utilisateurs ne visitent votre site que pour trouver un numéro de téléphone ou une adresse. Assurez-vous qu'ils ne perdent pas une heure à les dénicher. Quelques bonnes solutions pour cela:

$\odot$ ajouter dans le menu un intitulé « Contact » ou «Informations pratiques»;

$\odot$ inclure l'information dans la bannière du site, c'est-à-dire le bandeau qui occupe la partie haute de la page, en haut à droite;

$\odot$ répéter cette information dans le pied de page.

\section{La recherche}

Pouvoir faire une recherche sur le contenu d'un site web est un service classique. Assurez-vous que les fonctionnalités de recherche de votre site soient toujours au même endroit sur toutes les pages - quelque part dans la barre de navigation ou en haut de chaque page, là où les utilisateurs s'attendent à trouver les options de recherche. Ce qui complique les choses sur un site web de bibliothèque, c'est que les usagers veulent aussi pouvoir interroger le catalogue ou l'outil de découverte. Si votre site et votre catalogue peuvent être interrogés depuis une même fenêtre de recherche, c'est parfait! À défaut, faites de votre mieux pour faciliter leur cohabitation.

\section{Une arborescence cohérente}

Comme dans votre bâtiment, les utilisateurs doivent savoir où ils se trouvent sur votre site web, ce qu'ils peuvent faire et comment passer facilement d'un endroit à l'autre. Une arborescence cohérente est importante, car elle permet aux utilisateurs de ne pas se perdre. 
Souvent, l'arborescence des sites de bibliothèques devient incohérente parce que le catalogue lui-même, des blogs à part ou des microsites ont été ajoutés au site principal. Ces sites satellites ont souvent une arborescence et un aspect visuel différents. Cliquer dans une interface et être envoyé vers un environnement complètement différent peut désorienter les utilisateurs. Ce sentiment réduit leur confiance - en eux-mêmes et dans le site - et les empêche de faire sereinement ce qu'ils ont à y faire.

Malheureusement, la construction d'un site morceau par morceau est fréquente, parce que c'est le moyen le plus simple d'ajouter du contenu. Au lieu de prendre le temps d'insérer le nouveau contenu dans un environnement plus large - une structure organisée -, il est plus rapide d'en créer un nouveau et de faire le lien depuis le site principal.

Le catalogue de la bibliothèque est un autre élément qui reste, encore trop souvent, visuellement distinct du site web en luimême. Là encore, la transition entre une interface et une autre est perturbante. De plus en plus de bibliothèques ont traité le problème. Allez visiter les sites web de la Darien Library (<www. darienlibrary.org $>$ ) et la Topeka et Shawnee County Public Library (<http://tscpl.org $>$ ) pour voir des exemples de catalogues bien intégrés au site web. En France, le site de la bibliothèque de l'université de Rennes 2 gère bien la chose.

\section{Réduire le nombre d'arborescences}

Pour simplifier l'interface et éviter la multiplication des arborescences parallèles, il est préférable de supprimer les contenus externes et de les intégrer dans le site principal. Hormis les cas très particuliers des fonds anciens ou d'un projet très spécifique (exposition virtuelle, carnet de recherche), si un élément n'est pas assez important pour être intégré dans l'arborescence, c'est que son contenu n'en vaut pas la peine. 


\section{À vous de jouer}

Votre site utilise la majorité de ces conventions: 10 points.

Votre site utilise la moitié de ces conventions: 5 points.

Seulement quelques-unes: 0 point.

Utilisez les différents items de l'autodiagnostic comme un guide d'action, et modifiez votre site web de manière à ce qu'il respecte les conventions ci-dessus.

\subsection{Votre page d'accueil exprime clairement ce que l'on peut faire sur votre site}

\section{Pourquoi c'est important}

\section{Difficulté **}

Les internautes sont souvent impatients et ont tendance à porter des jugements hâtifs et définitifs sur la qualité d'un site. S'assurer que votre page d'accueil envoie un message clair est un moyen efficace d'améliorer la première impression produite sur vos visiteurs. Votre page d'accueil peut par exemple signifier: «Voici tout ce que nous faisons pour vous» ou «Voici tout ce que vous pouvez faire sur ce site». Cela participe de la valeur de votre site et met vos utilisateurs en confiance.

Une page d'accueil bien conçue fait plus qu'influencer la perception des internautes. Elle augmente aussi l'utilisabilité du site en les amenant directement là où ils veulent aller. Le design de la page d'accueil contribue donc à l'utilisabilité générale de votre site.

La figure 7.5 montre une page au visuel extrêmement surprenant. L'image est nouvelle, la marque est intrigante et l'impression globale que vous retirez de cette page d'accueil est que la bibliothèque est un lieu plein de vie. Malheureusement, ça s'arrête là. 


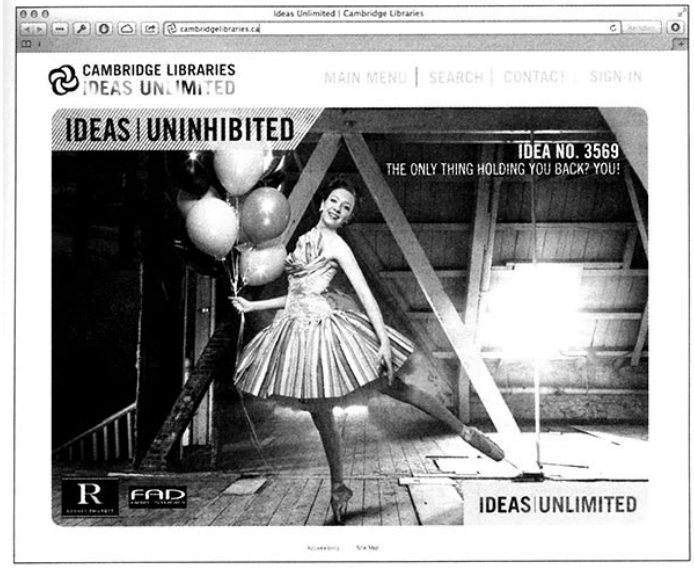

Fig 7.5 Page d'accueil de la bibliothèque municipale de Cambridge (Canada)

C'est beau, mais les quatre rubriques disponibles sur la page ne suffisent pas. L'intitulé Main menu (menu principal) cache toute l'arborescence du site! Cela demande aux utilisateurs de cliquer une fois de trop. Plus grave, cela les empêche de savoir instantanément ce qu'ils vont pouvoir faire sur le site.

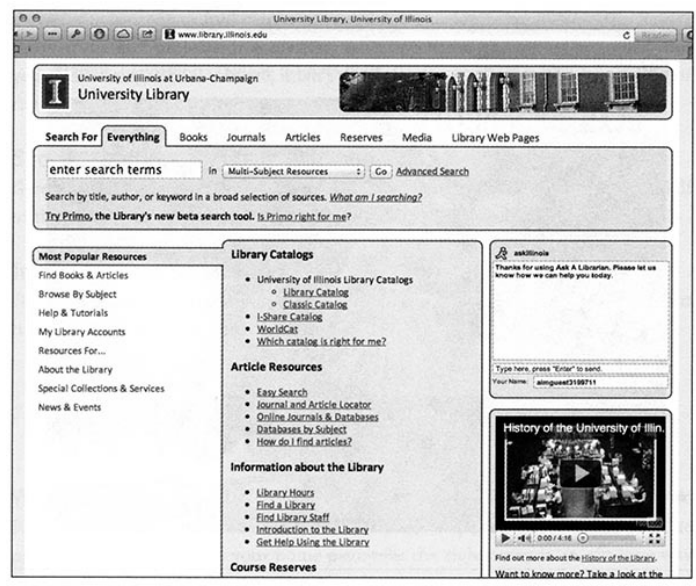

Fig 7.6 Page d'accueil de la bibliothèque universitaire de l'Illinois 
La figure 7.6 montre une page d'accueil chargée qui rend difficile la compréhension de l'objectif du site en proposant trop d'informations. Si la page est certainement l'expression de ce qu'on peut faire sur le site, elle est confuse. Parmi tous ces liens, certains sont utiles, mais ils sont étouffés par l'information superflue. Cette page gagnerait à mettre en valeur les services les plus importants.

La figure 7.7 montre une page visuellement attirante qui dégage une impression classique et typiquement universitaire. Elle propose plusieurs possibilités d'action. Les intitulés de l'arborescence sont bien mis en valeur et faciles à comprendre: Find (Trouver), Request (Demander), Help (Demander de l'aide). Le champ de recherche est bien visible. Cette page d'accueil est aérée et permet aux gens d'aller là où ils veulent.

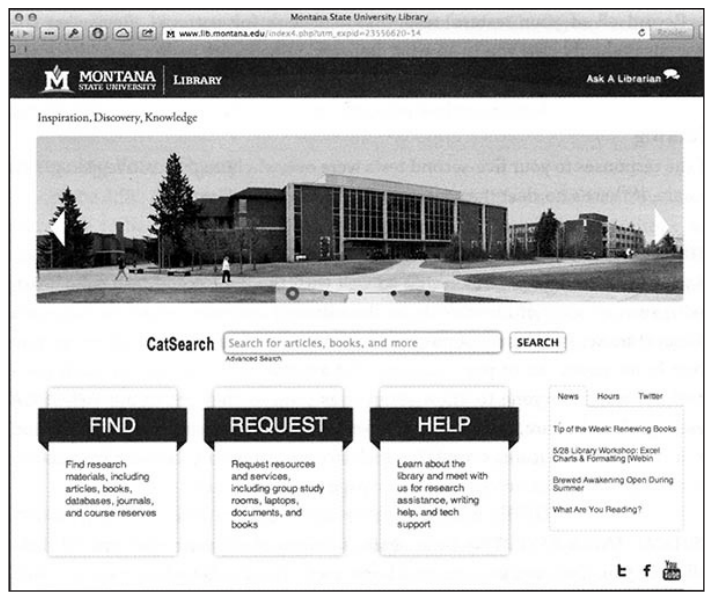

Fig. 7.7 Page d'accueil de la bibliothèque universitaire de l'État du Montana

\section{Autodiagnostic}

Un bon moyen de déterminer si votre page d'accueil délivre un message clair est de conduire un test de cinq secondes (voir 
chapitre 2). Il s'agit d'un test d'utilisabilité pendant lequel les testeurs regardent une page web pendant (devinez!) cinq secondes. Vous leur demandez ensuite de se souvenir de ce qu'ils ont vu sur la page.

Quand vous menez le test de cinq secondes pour savoir ce que dit votre page d'accueil, faites bien attention aux questions que vous posez aux testeurs. Si vous demandez: "Que pouvez-vous faire sur cette page?», vous en dites trop. Demandez plutôt: «De quoi vous souvenez-vous à propos de cette page?» Peut-être les utilisateurs ne se souviendront-ils que de certaines images ou couleurs ou de certains mots. Mais s'ils décrivent certaines des choses que l'on peut faire sur le site, c'est l'idéal! C'est à coup sûr le signe que votre page communique de manière efficace.

C'est seulement quand votre testeur aura fini de répondre à votre question initiale que vous pourrez aller plus loin en lui demandant: «Que pouvez-vous faire sur cette page?» À ce stade, vous pouvez les aider un peu. Passer en revue les réponses devrait vous éclairer sur l'impression que produit votre site et vous montrer où porter vos efforts.

Si le test de 5 secondes donne des résultats très positifs: 10 points.

Si l'objectif du site n’a pas été clairement exprimé: 0 point.

\section{Passez à l'action et créez une page d'accueil efficace}

Si la page d'accueil de votre bibliothèque n'a pas réussi le test de cinq secondes, ne vous laissez pas abattre: toutes sortes de bibliothèques, des plus modestes aux plus grandes, font l'erreur de proposer trop d'options et de contenu sur leur page d'accueil par pur souci de bien faire. La bibliothèque fait tant de choses qu'il lui faut les montrer à tous dès l'arrivée sur son site! Un noble objectif, bien sûr, mais comme le prouve le test de cinq secondes, trop d'informations sur une page d'accueil noient les 
plus importantes et l'impression dominante est celle d'un fouillis intimidant et cryptique. Voici quelques stratégies pour créer une page d'accueil efficace.

\section{Les fonctionnalités essentielles revisitées}

Une fois de plus, pensez aux fonctionnalités essentielles de votre site. Comparez votre page actuelle avec la liste des fonctionnalités essentielles que vous avez réalisée au point de repère 7.2. Votre page d'accueil ne doit pas se contenter de les rendre accessibles; elle doit les rendre VRAIMENT très accessibles. Vous ne pouvez pas vous satisfaire d'un simple lien vers votre catalogue ou votre outil de découverte. Vous devez proposer une fenêtre de recherche bien en évidence, qui occupe le centre de la page.

\section{Soyez fier de votre marque}

Quand vous pensez à votre page d'accueil, pensez aussi à donner aux utilisateurs une idée de ce que fait la bibliothèque en général. Vous pouvez faire cela en affichant votre marque (ce que les bibliothèques de Cambridge, évoquées plus haut, font bien) et en mettant en avant les événements que vous organisez.

\subsection{Le site s’adapte à tous les écrans}

\section{Pourquoi c'est important}

\section{Difficulté $* * *$}

Avez-vous pris récemment le train ou l'avion? Les cartes d'embarquement et les billets électroniques, la myriade de dispositifs divertissant les voyageurs et le wi-fi à bord sont là pour rappeler l'ubiquité toujours plus grande du Web et la diversité des appareils qui permettent de s'y connecter.

Qu'on le veuille ou non, les sites web de bibliothèques prennent part à cette évolution. Regardez les statistiques de votre site web. 
Il est certain que les connexions depuis un smartphone ou une tablette sont désormais plus courantes et ont augmenté d'une année sur l'autre. Concevoir des services en ligne destinés aux individus assis derrière des bureaux avec un ordinateur, c'est rester aveugle aux besoins et aux pratiques de vos utilisateurs.

\section{À vous de jouer}

Il ne vous sera pas difficile de tester votre site web sur différents appareils. Vous n'aurez même pas à en acheter pour pouvoir le tester. Souvenez-vous que ces dispositifs sont omniprésents: il y a donc de bonnes chances pour que vous puissiez vous débrouiller avec ceux du personnel de la bibliothèque. Au minimum, vous pourrez tester votre site sur iOS (iPhone, iPad) et sur quelques appareils utilisant le système d'exploitation Android. Les navigateurs de ces dispositifs réussissent généralement bien à redimensionner les sites web. Cliquez et explorez votre site. Pouvez-vous facilement accéder aux principales fonctionnalités?

Ce diagnostic ne fait pas dans la nuance: c'est tout ou rien.

Votre site web est conçu pour les smartphones et tablettes ou développé en Responsive Web Design (c'est-à-dire dimensionne l'affichage automatiquement en fonction du type d'appareil utilisé et de la taille de l'écran): 10 points.

Il ne s'affiche pas correctement et ne peut être utilisé que sur un ordinateur de bureau? 0 point.

\section{Passez à l'action et mettez en place du Responsive Web Design (RWD)}

Les navigateurs de smartphone réussissent habituellement bien à afficher les sites web. Dans l'idéal, il est préférable que votre site soit conçu, dès le départ, pour bien s'afficher sur un téléphone 
mobile. Une bonne pratique, désormais courante, est le recours au Responsive Web Design (RWD).

Avec le RWD, les sites sont redimensionnés correctement en fonction de l'appareil sans devoir tenir compte de la résolution de l'affichage. Cela permet d'optimiser le rendu visuel et de réduire le nombre de défilements, de balayages, de pincements et de zooms que nécessite souvent l'utilisation d'un site web sur un mobile. Observez les figures 7.8 et 7.9 pour voir comment le RWD gère différentes tailles d'écran.

Ce chapitre n'entrera pas dans les détails techniques à propos du RWD, car vous trouverez pléthore d'informations à ce sujet sur le Web. L'article Responsive Web Design d'Ethan Marcotte < http:// alistapart.com/articles/responsive-web-design >, qui a été le premier à utiliser ce terme, est un bon point de départ. Voir la section «Lectures en plus» à la fin de ce chapitre.

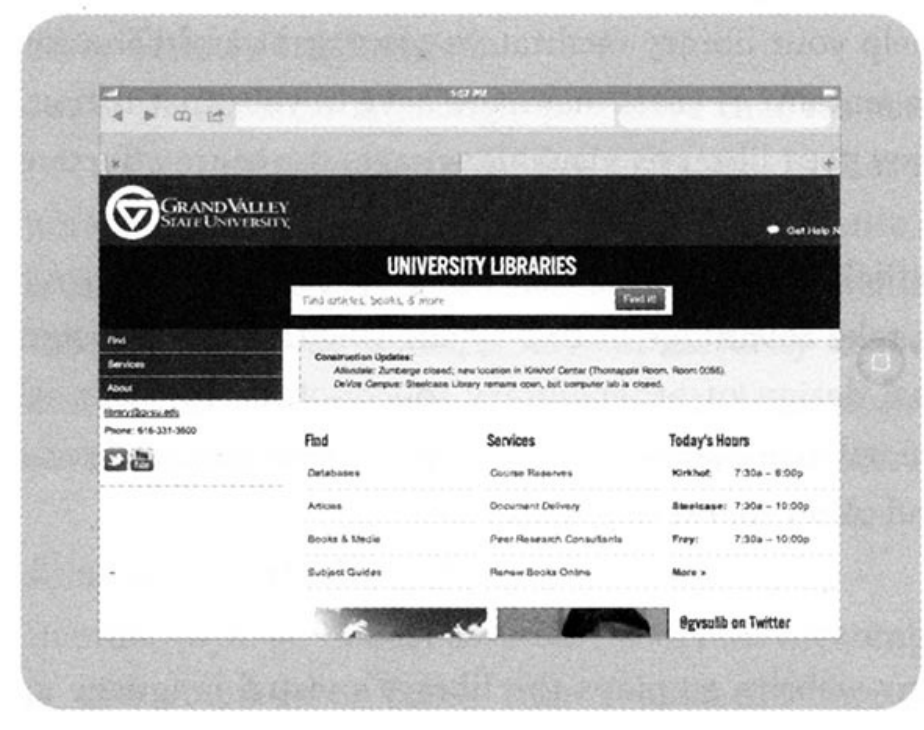

Fig. 7.8 Page d'accueil de bibliothèque sur iPad 


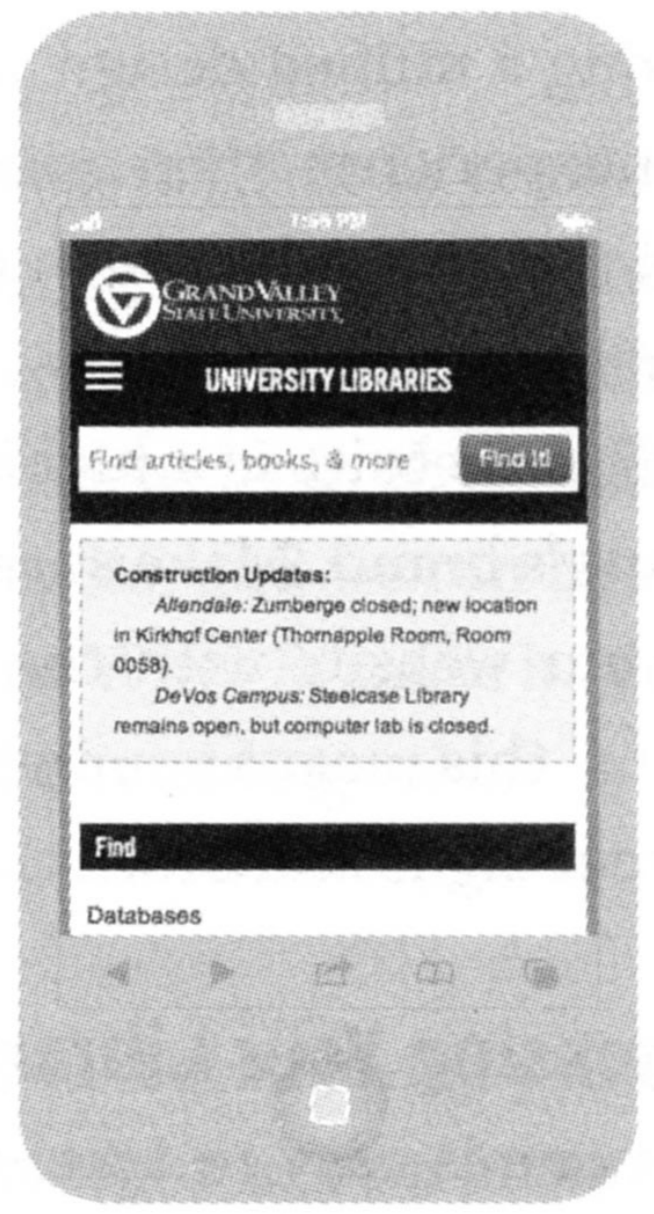

Fig. 7.9 Page d'accueil de bibliothèque sur iPhone

\section{Les limites actuelles du Responsive Web Design}

S’il est assez facile de concevoir des sites web en RWD, la marge de manœuvre des bibliothèques s'arrête là. Les applications tierces, les OPAC pour le catalogue et plus encore les bases de données et les bouquets de journaux en ligne ne s'affichent pas toujours bien sur smartphone. C'est un gros problème. À part 
faire pression sur les fournisseurs pour qu'ils adoptent à leur tour le RWD, il n'y a pas encore de solution pratique pour améliorer la situation. Heureusement, certains ont bien compris cette évolution et adaptent enfin leurs produits.

\subsection{Le site web utilise l'identité visuelle de la bibliothèque}

\section{Pourquoi c'est important}

\section{Difficulté $* * *$}

À la fin de l'autodiagnostic 6.1, vous avez fait la liste de tous les endroits où vous utilisiez votre identité visuelle. Il vous était recommandé d'y ajouter le site web. Ceci est un rappel.

Il est important que tous les points de contact de la bibliothèque aient une apparence similaire. Utiliser un même langage visuel aidera vos utilisateurs à avoir des repères cohérents.

En diffusant une même identité visuelle reconnaissable en tout point, vous construirez un sentiment d'appartenance et de familiarité qui vous permettra d'accroître le nombre de vos utilisateurs.

Votre site web est un point de contact très fréquenté qui peut vous aider à installer l'identité visuelle de votre bibliothèque. Pour être sûr qu'il joue bien ce rôle, utilisez la même charte graphique pour votre site web que pour les autres points de contact de la bibliothèque. En pratique, cela signifie que votre site web doit utiliser la même palette de couleurs, la même police et le même ton que celui des brochures et autres supports de communication.

\section{Estimez votre CUTI}

Voilà un autodiagnostic facile à faire. Soit votre site web utilise l'identité visuelle de votre bibliothèque, soit il ne l'utilise pas. 
Le diagnostic est aisé, le traitement ne l'est pas. La condition préalable, et non des moindres, est d'avoir une identité visuelle (cf. chapitre 6), que vous pouvez appliquer à votre site web. Une fois que ce sera fait, votre site web ressemblera à vos documents imprimés et à votre signalétique.

Encore un score facile à calculer.

Si votre site web a la même apparence que les autres produits de la bibliothèque: 5 points.

Sinon: 0 point.

\section{Mettez en œuvre l'identité visuelle de la bibliothèque}

Pour améliorer votre score après ce diagnostic, vous aurez très probablement à développer votre identité visuelle. Vous devrez probablement prévoir une refonte de votre site web. Cela prendra beaucoup de temps et demandera de gros efforts. Mais c'est absolument nécessaire si vous voulez créer une expérience vraiment exceptionnelle pour vos utilisateurs.

\subsection{Vous utilisez les réseaux sociaux à bon escient}

\section{Difficulté *}

Votre présence en ligne ne se résume sans doute pas à votre site web. Elle comprend tous les autres outils web que vous utilisez pour contacter, informer et inviter vos utilisateurs à venir. Cet autodiagnostic vous permettra de voir si vous mettez en œuvre ces différents outils de manière raisonnée et utile à la communauté que vous desservez.

\section{Pourquoi c'est important}

Les médias sociaux sont désormais si omniprésents qu'on ne se souvient plus de ce qui s'est passé lorsqu'ils ont émergé, autour 
de 2010. Au début, bien des bibliothèques, pour ne pas rater le train en marche, ont sauté à bord avec impatience: elles ont créé un compte ou une page sur tous les réseaux sociaux (Facebook, Twitter, Foursquare, etc.), en espérant que leurs utilisateurs y seraient et viendraient les y chercher. Heureusement, les médias sociaux ont maintenant atteint un niveau de maturité qui permet d'être plus sélectif et de mieux savoir où porter les efforts. Aujourd'hui, il ne s'agit plus d'être présent à tout prix sur les réseaux sociaux pour être à la page, mais d'y être actif à condition que cela apporte vraiment quelque chose à vos utilisateurs.

\section{À vous de jouer}

Pour mesurer de manière tangible l'intérêt de votre présence sur les réseaux sociaux, le meilleur moyen est d'aller interroger les membres de votre communauté. Organisez une courte enquête sur la présence de la bibliothèque dans les trois principaux réseaux sociaux. Vous pouvez constituer «le top 3 » comme bon vous semble: par exemple, les trois médias sociaux sur lesquels vous investissez le plus de temps et d'efforts. Demandez alors à vos utilisateurs s'ils savent que vous avez un compte. Votre enquête pourrait ressembler à cela:

Savez-vous que la bibliothèque est sur:

a. Facebook? (oui/non)

b. Twitter? (oui/non)

c. Pinterest? (oui/non)

Si vous obtenez trois oui: 5 points; si vous avez deux oui: 2 points; si les non l'emportent: 0 point.

\section{Passez à l'action et donnez du sens à votre présence sur les réseaux sociaux}

Vous pouvez avoir un mauvais score sur ce point pour plusieurs raisons. Peut-être que vos utilisateurs ne connaissent pas votre 
page Facebook; peut-être ne sont-ils pas du tout sur Pinterest. Quelle qu'en soit la raison, lisez la suite: vous y trouverez des idées pour donner du sens et du poids à votre présence sur les réseaux sociaux.

\section{Faites-vous connaître}

Vous devez faire connaître votre présence sur les réseaux sociaux comme vous faites connaître tous les services offerts par la bibliothèque. Vous pouvez tout simplement mettre des liens vers les comptes Facebook ou Twitter (ou autres) sur des supports imprimés comme les marque-pages, et promouvoir ces comptes sur la page d'accueil du site de votre bibliothèque.

\section{Focalisez vos efforts en fonction des pratiques} de votre communauté d'utilisateurs

Faites une enquête auprès de vos utilisateurs pour savoir où ils passent leur temps en ligne. Si vous avez une forte communauté d'utilisateurs de Twitter, la bibliothèque tirera un avantage évident à ouvrir un compte sur Twitter. S’ils préfèrent Facebook, ouvrez une page Facebook. Vous avez plus de chance de constituer une communauté en ligne représentative si vous utilisez le réseau social préféré de la majorité de vos utilisateurs pour interagir réellement avec eux.

Analysez la communauté actuelle des gens qui suivent les comptes de votre bibliothèque sur les réseaux sociaux. Si elle est composée en majorité de bibliothécaires, reprenez depuis le début.

\section{Augmentez le facteur d'engagement}

Avec un peu de recul sur les réseaux sociaux, vous avez probablement déjà pris conscience qu'il est réducteur et contre-productif de les utiliser comme outil de diffusion d'information à sens unique de la bibliothèque vers ses utilisateurs. Faites preuve de bon sens: utilisez la puissance de Facebook ou de Twitter de 
manière opportune: ce n'est pas un mur d'affichage de vos informations, mais un lieu d'échanges qui vous permet de poser des questions aux internautes, d'engager le dialogue avec eux, de répondre à leurs besoins d'information, voire d'encourager les échanges entre eux au sujet de la bibliothèque.

Impliquez les membres de votre communauté

dans le développement de votre présence sur les réseaux sociaux

Si vous avez des difficultés à obtenir la participation que vous souhaitez, vous devriez essayer d'impliquer vos utilisateurs dans le développement de votre présence sur les réseaux sociaux. Par exemple, pourquoi ne pas laisser certaines personnes twitter pour le compte de la bibliothèque? Ou bien, si vous êtes dans une bibliothèque universitaire, pourquoi n'impliquez-vous pas les représentants étudiants dans votre présence sur Facebook? Ce type d'action peut stimuler la participation de vos utilisateurs de manière remarquable.

\subsection{Plus de lectures}

Center for Plain Language. [En ligne] < http://centerforplainlanguage.org >.

Krug S., Don't Make Me Think: A Common Sense Approach to Web Usability, $3^{\text {rd }}$ ed., Berkeley, New Riders, 2005.

Krug S., Rocket Surgery Made Easy: The Do-It Yourself Guide to Finding and Fixing Usability Problems, Berkeley, New Riders, 2009. Nielsen J., « Guidelines For Visualizing Links », mai 2004. [En ligne] <www.nngroup.com/articles/guidelines-for-visualizing-links >.

Reddish G., Letting Go of the Words: Writing Web Content That Works (Interactive Technologies), 2nd ed., Waltham, Morgan Kaufmann, 2012.

Reidsma M., «How We Do Usability Testing», novembre 2011. [En ligne] <http://matthew.reidsrow.com/articles/13>. 


\section{Chapitre 8. La bibliothèque comme outil de travail}

Ce livre a jusqu'ici porté sur des points de contact précis et vous a proposé de travailler sur les interactions qui ont lieu lorsque les usagers utilisent les bâtiments et certains services: signalétique, procédures et règlements, espaces d'accueil, sites web et espaces physiques.

Ces personnes interagissent avec les services de la bibliothèque de bien d'autres manières, notamment lorsqu'ils utilisent, entre autres, les ordinateurs publics, l'infrastructure technologique, les collections ou les animations proposées. Ce chapitre aborde ces autres types d'interactions et propose quelques idées pour améliorer l'expérience des utilisateurs de ces services.

\subsection{Les équipements et appareils proposés dans la bibliothèque sont adaptés aux besoins, utiles et utilisables}

\section{Difficulté $* *$}

Il fut un temps où vous pouviez entrer dans une bibliothèque, trouver ce que vous vouliez lire, faire ce que vous vouliez y faire et rentrer chez vous en emportant tout un tas de choses, le tout sans jamais avoir à utiliser le moindre appareil. Même si c'est peut-être encore le cas, dans quelques rares occasions particulières (vraiment très, très rares), vous auriez du mal à trouver quelqu'un dans votre bâtiment qui n'ait pas eu à se servir d'un équipement sur place, qu'il s'agisse d'un ordinateur public pour faire une recherche dans le catalogue, de votre réseau wi-fi via son ordinateur, son smartphone ou sa tablette, etc. 
La vie actuelle est totalement irriguée par la technologie: ainsi, si votre bibliothèque n'a pas encore engagé de réflexion sur l'expérience des personnes qui utilisent les équipements qu'elle propose, il est temps d'y songer.

\section{Pourquoi c'est important}

Prenez une minute pour réfléchir aux différents équipements que vous offrez dans votre bâtiment. En voici une liste non limitative:

$\odot$ ordinateurs publics;

$\odot$ ordinateurs portables;

$\odot$ tablettes;

$\odot$ photocopieurs et imprimantes;

$\odot$ scanners;

$\odot$ lecteurs de DVD;

○ télévisions ou écrans;

$\odot$ vidéoprojecteurs;

$\odot$ antivol;

○ câbles RJ45;

$\odot$ distributeurs divers;

$\odot$ lecteurs / tireurs de microfiches;

$\odot$ automates de prêt/retour;

$\odot$ wi-fi;

$\odot$ liseuses;

$\odot$ lecteurs MP3 ou dictaphones;

$\odot$ consoles de jeux;

$\odot$ appareils photo et caméras vidéos. 
Cela vous oblige à prendre en compte de nombreux objets techniques et ce n'est qu'un début: vous avez probablement bien d'autres choses à ajouter à cette liste, tout particulièrement si vous prêtez du matériel électronique ou gérez des paiements monétiques ou en ligne.

Si vous pensez à la masse de ces équipements, et que vous vous demandez comment être sûr qu'ils sont tous faciles à utiliser, vous pouvez légitimement vous sentir dépassé! Avant tout, réfléchissez à ce que ressentent vos usagers en utilisant tous ces matériels. Mettez-vous à leur place, et imaginez-vous mis au défi de comprendre comment ils fonctionnent, comme ils sont sans doute obligés de le faire à chaque fois qu'ils passent les portes de la bibliothèque. Si vous adoptez ce point de vue, vous ne pourrez qu'être agréablement surpris que les usagers viennent encore à la bibliothèque, malgré les pièges qui leur sont tendus à chaque fois qu'ils doivent utiliser vos équipements!

\section{À vous de jouer}

Quels sont ces pièges et comment les évaluer? Il y a beaucoup de choses à prendre en compte dans cet autodiagnostic et il ne sera sans doute pas facile d'avoir une approche globale des équipements que vous proposez, ni de ce que ressentent vos usagers lorsqu'ils utilisent le matériel mis à leur disposition dans vos espaces. Commencez donc par évaluer de petites choses.

Tout d'abord, votre wi-fi. Est-ce facile de s'y connecter? Voici un bon test: si votre site web consacre une page à expliquer comment se connecter au wi-fi, c'est qu'il y a quelque chose qui ne va pas. Oubliez les codes-barres, oubliez les mots de passe. Dans l'idéal, les visiteurs doivent juste avoir à sélectionner votre réseau pour être connectés. Si accéder à votre wi-fi est aussi simple que ça, marquez 10 points. Sinon, 0 point. 
Prenez ensuite l'impression sur place. Imprimer est-il facile pour vos usagers? Commencez par détailler toutes les étapes nécessaires pour qu'un utilisateur imprime un document. Doit-il acheter des unités sur une carte spéciale et ensuite aller sur un ordinateur différent pour lancer l'impression? Là, déjà, on peut parler de démarche compliquée.

Une autre manière d'évaluer le niveau de difficulté est de faire un comptage aléatoire du nombre de questions sur l'impression auxquelles doivent répondre les collègues en service public. Faites un relevé pendant deux semaines. Si après deux semaines, moins de $10 \%$ des questions posées portent sur les impressions, marquez 10 points. Si ces questions dépassent $10 \%$, 0 point.

Enfin, prêtez attention aux autres équipements en libre-service, comme les scanners ou les automates de prêt/retour. Les deux types de matériel peuvent être délicats à utiliser: il serait présomptueux de penser qu'ils sont suffisamment intuitifs pour être utilisés sans aide. La question est la suivante: avez-vous fourni des instructions simples et faciles à suivre pour ces machines? Si vos scanners ou automates de prêt ont des modes d'emploi simples et faciles à trouver, marquez 5 points. Si ce n'est pas le cas, marquez 0 point.

Cet autodiagnostic compte pour 25 points au maximum. Additionnez votre score et reportez-le dans le tableau de résultats en annexe.

\section{Passez à l'action et facilitez l'usage de vos équipements}

Le matériel est une préoccupation majeure pour les bibliothèques et, du fait de la multitude d'objets technologiques présents dans les espaces, il n'y a pas de méthode simple pour faciliter la vie des gens. Sans remettre en cause ce constat de départ, il paraît possible d'apporter de multiples petites améliorations aux 
environnements techniques pour faciliter l'expérience de l'utilisateur. En voici une liste non exhaustive:

\section{Imprimer et photocopier est facile}

L'envie est forte de suggérer l'abandon de tous les systèmes de paiement pour les copies et impressions publiques dans les bibliothèques, afin d'améliorer l'expérience de l'usager: les restrictions budgétaires en décident autrement. Il est impossible pour de nombreuses bibliothèques d'échapper aux contraintes de l'impression payante, et il faut faire avec. Certains freins peuvent être levés, en proposant par exemple un service gratuit pour de petits travaux inférieurs à cinq pages. Avez-vous déjà eu affaire à une personne pressée qui a besoin d'imprimer une page ou deux et s'énerve d'avoir à acheter une carte de photocopie pour si peu? Si oui, vous êtes probablement convaincu de l'intérêt d'une telle règle. Un autre service à envisager est l'impression en réseau (Cloud printing). Comme le montrent certainement les statistiques de votre établissement, de plus en plus d'utilisateurs apportent leur propre matériel à la bibliothèque: proposer une manière d'imprimer depuis leur ordinateur à eux est donc une solution logique, même si elle change, dans un premier temps, bien des routines et des habitudes.

\section{Il est facile d'utiliser le wi-fi}

Dix points à toute bibliothèque qui propose un réseau wi-fi qui n'exige pas de code d'accès ou de code-barres. Zéro point aux bibliothèques qui imposent un mot de passe pour se connecter au wi-fi. Pourquoi cela? Ne pas avoir à s'identifier pour se connecter au wi-fi est plus facile. Point à la ligne.

Bien sûr, tout cela dépend du type de bibliothèque dans laquelle vous travaillez: un réseau wi-fi ouvert peut ne pas être envisageable. Demandez-vous pourquoi vous imposez aux utilisateurs de s'identifier pour accéder au wi-fi et pensez sérieusement à 
revenir sur cette règle si vous en avez la possibilité. Et si vous trouvez une solution dans votre environnement, partagez-la avec le reste de la communauté professionnelle. Votre expérience réussie peut permettre d'apporter des arguments valables dans d'autres contextes.

Votre environnement informatique n'est pas inutilement verrouillé

Gérer un parc d'ordinateurs publics n'est pas chose aisée. Les utilisateurs peuvent faire bien des choses sur ces ordinateurs, du téléchargement de logiciels indésirables à l'introduction délibérée de malwares ou de virus sur le réseau: c'est un défi pour les services informatiques. Les ordinateurs en accès libre peuvent devenir un cauchemar pour celui qui les administre au quotidien. Cela dit, résistez à la tentation de tout verrouiller, au point que personne ne puisse plus les utiliser en fonction de son besoin. Il s'agit d'une frontière ténue, et chaque bibliothèque devra se faire sa propre idée sur l'endroit où passe cette ligne. Un adage dit que «la quantité de travail pour prévenir doit être équivalente à la quantité de travail pour guérir». S'en souvenir peut permettre de résister à la tentation de rendre l'accès aux ordinateurs trop restrictif et d'éviter que la frustration des utilisateurs ne dépasse le gain en termes de stabilité et de facilité d'entretien.

\section{Votre environnement informatique propose ce que vos usagers} utilisent et préfèrent

Les deux principaux systèmes d'exploitation sont Windows et Mac OS. Votre bibliothèque propose-t-elle un environnement tout Windows ou tout Mac? Dans les deux cas, cela pose le même problème: vos usagers sont obligés d'utiliser un système d'exploitation qui ne leur est pas familier. C'est au mieux inconfortable, au pire frustrant. Proposer un accès à chaque environnement et 
permettre aux gens de choisir celui qu'ils préfèrent est un signe d'attention à leurs besoins et préférences.

Le matériel en libre-service est intuitif à utiliser ou est accompagné d'un mode d'emploi facile à suivre

Vos scanners, lecteurs de microfiches, automates de prêt/retour, photocopieurs, imprimantes et autres machines en libre accès sont-ils accompagnés d'un mode d'emploi facile à trouver et facile à suivre? Si ce n'est pas le cas, mettez-vous au travail pour en créer. Quand vous n'avez aucun contrôle sur l'ergonomie de base d'un matériel (et c'est souvent le cas quand il s'agit d'imprimantes ou de scanners), la moindre des choses à faire pour faciliter la vie des gens est de fournir des modes d'emploi clairs et simples.

Impliquez les usagers dans le choix des équipements

Si vous avez mis en place un groupe d'usagers-conseil, pourquoi ne pas leur demander de l'aide lorsqu'il s'agit d'acheter de nouveaux matériels? Qu'il s'agisse de nouveaux ordinateurs, de scanners, d'automates de prêt/retour ou de matériel en prêt comme des portables, tablettes ou liseuses, ce sont les usagers qui vont être les premiers à utiliser ces équipements. Pourquoi ne pas leur demander leur avis avant d'acheter?

\subsection{Les collections sont adaptées aux besoins des utilisateurs}

\section{Difficulté **}

Le titre de cette partie n'est-il pas le principe numéro 1 de toute bibliothèque? Vous devez avoir dans vos collections des choses que les membres de votre communauté veulent lire, regarder, écouter ou utiliser d'une manière ou d'une autre. Point final. 
Attendez! Vous n'êtes pas en train de lire l'introduction d'un manuel de politique documentaire; vous réfléchissez au fait d'être attentifs aux besoins des utilisateurs au moment où vous construisez votre collection. À moins de travailler dans une bibliothèque où $100 \%$ des collections circulent régulièrement, vous allez probablement continuer votre lecture.

\section{Pourquoi c'est important}

Un végétarien entre dans un restaurant qui vient d'ouvrir dans son quartier et jette un œil à la carte. Ne trouvant rien pour lui, si ce n'est une simple salade, notre végétarien tourne les talons et va manger dans son bistrot préféré, où il sait qu'il aura une carte variée à sa disposition. Retournera-t-il le lendemain dans ce restaurant? Non. L'année prochaine? C'est peu probable. Pourquoi? Parce qu'il a vu qu'il ne pouvait rien y manger.

Vous voyez où veut en venir cette histoire à la morale transparente. Si les collections de votre bibliothèque ne répondent pas aux besoins de votre communauté, quels qu'ils soient, de moins en moins de personnes utiliseront votre bibliothèque. Mais ce n'est pas aussi simple: même si vous desservez une communauté homogène, ses besoins peuvent être très divers, de sorte que vous répondez probablement à certains, mais peut-être pas à tous. Vous pouvez sûrement mieux faire, mais avant de voir comment, calculez votre CUTI.

\section{À vous de jouer}

Pour déterminer si vos collections répondent aux besoins des membres de la communauté que vous desservez, vous pouvez faire deux choses: un sondage et un focus group.

Pour le sondage, ciblez chaque personne qui emprunte un livre de la bibliothèque pendant une période donnée (une semaine 
ou deux peuvent suffire). Distribuez des sondages au bureau de prêt ou ajoutez l'adresse d'un sondage en ligne sur leurs tickets de prêt (particulièrement utile si vous utilisez des automates de prêt). Dans votre sondage, posez deux questions:

$\odot$ citez un document que vous avez emprunté aujourd'hui;

$\odot$ est-ce que ce document correspondait;

$\odot$ parfaitement à ce que vous étiez venu chercher?

$\odot$ pas tout à fait, mais il porte sur le même sujet;

$\odot$ il n'a aucun rapport avec ce que j'étais venu chercher.

Comme vous le voyez, ce questionnaire ne s'intéresse pas aux pourquoi - pourquoi n'avez-vous pas emprunté ce que vous étiez venu chercher? Pourquoi sortez-vous avec quelque chose qui n’a aucun rapport avec ce que vous recherchiez au départ? Cela vous donnera juste un aperçu de la manière dont votre collection répond aux besoins des uns et des autres, d'une façon délibérément peu scientifique.

Marquez 8 points si plus de la moitié des répondants à votre sondage ont choisi 2 (a), 4 points si la moitié a choisi 2 (b) et 0 point s'ils ont choisi 2 (c).

Pour constituer le focus group, recrutez 5 à 6 personnes et questionnez-les sur la façon dont les collections de la bibliothèque répondent à leurs besoins. Demandez-leur pourquoi elles utilisent la bibliothèque et si elles y trouvent des choses intéressantes à emprunter. Attention, la conversation ne doit pas se transformer en discussion sur la facilité ou la difficulté à trouver les documents, qu'il s'agisse du catalogue ou des espaces. Restez à un niveau plus général. Attribuez-vous 7 points si le résultat est tout à fait positif, 3 points si les avis sont plus souvent positifs que négatifs, et 0 point si vous avez entendu plus de commentaires négatifs que de commentaires positifs. 
Additionnez les points du focus group à ceux du sondage et notez le total sur 25 dans le tableau de résultats.

\section{Passez à l'action : la collection définie par les utilisateurs}

La plupart des bibliothèques ont tout juste la moyenne à cet autodiagnostic: certains besoins sont satisfaits, mais il y a encore une marge d'amélioration. Voilà quelques idées qui vous permettraient d'être davantage guidés par vos usagers dans votre manière de développer vos collections.

\section{Apprenez à connaître vos utilisateurs}

Sans doute disposez-vous déjà des informations démographiques sur la communauté que vous desservez, et c'est une très bonne chose. Sinon, commencez par cela. Toutefois, ces données sont un peu abstraites pour permettre de construire les collections dont votre communauté aurait besoin. Poussez votre recherche un peu plus loin en revenant au chapitre2, sur les méthodes de recherche, et mettez en œuvre les techniques suivantes pour mieux connaître votre communauté: essayez les interviews et les sondes culturelles (enquête ethnographique allégée). Aller au cœur des centres d'intérêt et des motivations de vos utilisateurs vous donnera une base solide sur laquelle vous pourrez vous appuyer pour développer les collections de votre bibliothèque.

Construisez des personas: utilisez les résultats de vos interviews et de vos sondes culturelles pour concevoir trois à cinq personas pour votre bibliothèque. Comme cela a déjà été dit à plusieurs reprises dans ce livre, les personas peuvent vous aider dans tous les aspects du design de service dans votre bibliothèque, y compris la construction de collections. Retournez au chapitre 2, section 2.2 pour en savoir plus sur cette technique. 


\section{Parlez à ceux qui n’utilisent pas la bibliothèque}

Les raisons pour lesquelles certaines personnes de votre communauté n'utilisent pas votre bibliothèque sont nombreuses: l'une d'elles est peut-être que la collection ne répond pas à leurs besoins. Sortez de votre bibliothèque, allez vers la population et essayez d'en savoir plus sur ceux qui ne viennent jamais, ni physiquement ni virtuellement. Vous habitez la même ville: profitez-en pour les rencontrer et tenter d'en savoir plus. Ou bien adoptez une approche raisonnée pour mieux connaître ceux qui n'utilisent pas la bibliothèque: installez une table dans un centre commercial et proposez aux passants de répondre à une enquête ou de venir discuter avec vous. Vous en apprendrez probablement encore plus sur eux que sur leurs besoins documentaires, ce qui est une bonne chose.

\section{Construire la collection en gardant \\ à l'esprit les membres de votre communauté}

L'un des lieux communs les plus répandus en matière de développement de collection est le Patron-driven acquisition (PDA), "acquisitions à l'initiative des lecteurs». Le processus étant encore rare en France, il n'a pas émergé d'acronyme ou de formule unifiée en français.

L'introduction du PDA fait bouger les lignes: d'une conception traditionnelle selon laquelle les bibliothèques doivent construire une collection à l'épreuve du temps, on passe à l'idée que les collections sont au service des besoins des utilisateurs de la bibliothèque, ici et maintenant.

La meilleure manière d'introduire la notion de PDA est de réserver une partie de son budget pour l'expérimenter et d'autoriser les utilisateurs à l'utiliser pour sélectionner les documents qu'ils veulent ou dont ils ont besoin. 
Le PDA fonctionne tant pour les achats de documentation papier que pour l'électronique. Quelques fournisseurs proposent d'ailleurs des moyens d'inclure une option PDA dans votre circuit d'acquisitions. Si vous êtes tenté par l'exploration de cette option, il y a une abondante bibliographie sur le sujet (voir «Plus de lectures » à la fin de ce chapitre pour quelques suggestions.)

\section{Utilisez les données de prêt entre bibliothèques (PEB)}

Si votre bibliothèque propose du PEB, l'étude des demandes de PEB est un bon moyen de savoir si les membres de votre communauté ont besoin de documents que votre bibliothèque ne possède pas. Regardez si elles révèlent des lacunes dans votre collection et achetez des titres pour les combler.

\section{Exploitez les statistiques d'utilisation des ressources en ligne}

Il ne faut pas se contenter d'acheter pour avoir des collections pertinentes: il s'agit aussi de garder ce que les lecteurs utilisent vraiment. Regardez vos statistiques d'utilisation de la documentation en ligne. En BU, l'installation et l'exploitation d'ezPAARSE peuvent vous permettre, si vous offrez un accès distant à la documentation électronique via un EZproxy, de recroiser les données de consultation et celles sur les laboratoires de recherche ou les filières pour bien cibler la communication vers les communautés utilisatrices et mesurer les usages respectifs des bases acquises par les organismes de recherche et celles mises à disposition par l'université.

Personne n'utilise une base de données très chère ? Faites-en une promotion bien ciblée pendant quelques semaines, puis mesurez son utilisation. Si les consultations restent basses ou diminuent encore, arrêtez l'abonnement et investissez l'argent économisé dans un autre projet. 


\subsection{Les supports de communication sont utiles}

\section{Difficulté **}

Toutes ces listes de livres, ces marque-pages, brochures et newsletters que vous créez ont-ils un autre intérêt que celui de s'accumuler un peu partout dans la bibliothèque, façon marché aux puces? Ce dépliant sur "Comment faire une recherche dans les bases de données de la BU?» sort-il encore de son présentoir? Peut-être est-ce le moment d'évaluer si vos documents marketing répondent vraiment aux besoins des publics à desservir.

Cet autodiagnostic ne traite pas de la mise en forme des documents, évoquée au chapitre 6 . Il ne s'agit pas non plus de désencombrer les espaces (voir le chapitre 3, section 3.2). Ici, l'idée est plutôt de vous proposer des moyens de tester l'utilité de vos documents de communication et vous aider à mieux faire.

\section{Pourquoi c'est important}

Si vous avez déjà patienté dans une salle d'attente en cherchant quelque chose d'autre à lire que des revues toutes froissées vieilles de cinq ans, vous avez une petite idée de ce que l'on ressent lorsqu'on est cerné par des lectures éventées sans intérêt. C'est peut-être ce que vivront vos utilisateurs s'ils entrent dans votre bibliothèque, se rendent au point d'information et se retrouvent face à des documents présumés «utiles» sommairement mis en pages, périmés et qui ne répondent à aucun de leurs besoins.

Le bibliothécaire aime aider: il a donc une tendance naturelle à créer ce genre de vademecum imprimé à la volée, supposé aider certains utilisateurs et répondre à leurs besoins du moment. Ce qu'il vaudrait mieux faire, plutôt que d'agir au coup par coup, c'est avoir une approche planifiée et raisonnée pour développer des documents d'aide qui répondent réellement à un besoin, en nombre très limité. 


\section{À vous de jouer}

Plusieurs cas de figure sont possibles:

$\odot$ vous avez hérité d'une collection de documents mis en place par vos prédécesseurs;

$\odot$ vous venez tout juste de vous voir confier la charge de gérer ce type de documents alors que le contenu de chacun d'entre eux reste sous la responsabilité d'un autre département ou d'une autre personne.

Que pouvez-vous faire dans ces cas-là?

Comme cela est proposé au chapitre 6, section 6.2 («Une même charte graphique pour toute la signalétique»), faites un audit des supports de communication de votre bibliothèque. Savoir exactement ce qu'elle produit, où ces documents sont distribués, s'ils sont en cohérence avec le site web, et qui est responsable de quoi vous aidera à vous faire une idée de la production et de sa pertinence.

Nous vous suggérons de créer un tableau à six colonnes et de collecter les informations suivantes:

$\odot$ lieu;

$\odot$ type de matériel (brochure, marque-page, newsletter, affiche, dépliant, etc.);

$\odot$ dernière mise à jour (date);

$\odot$ information également disponible sur le site web (oui / non);

$\odot$ conforme à l'identité visuelle (oui / non);

$\odot$ rédigé de manière appropriée (oui/non);

$\odot$ encore utile (oui/non).

Les trois premières colonnes sont faciles à compléter. Les trois suivantes («identité visuelle», «rédigée de manière appropriée» 
et "présent sur le site web») vont exiger une évaluation un peu plus poussée, qu'il faudra prendre le temps de faire.

Pour compléter la dernière colonne (utilité), un exercice très simple aide à mettre à contribution les membres de votre communauté: installez un grand tableau d'affichage dans un lieu de passage et épinglez tous vos documents dessus. Sous chacun d'entre eux, accrochez une feuille blanche. En haut du panneau d'affichage, posez une seule question: utilisez-vous ces documents?

Ne demandez pas si les gens les aiment bien ou pensent qu'ils sont utiles: demandez juste s'ils les utilisent. Vous ne voulez pas savoir si vos utilisateurs trouvent ces documents sympas ou utiles, mais seulement s'ils les utilisent ou non.

Laissez un marqueur à portée de main et demandez aux utilisateurs de faire un trait sous chaque document qu'ils utilisent. Laissez le tableau en place pendant deux semaines maximum (plus longtemps, tout le monde s'y sera habitué et plus personne ne le remplira). Au bout de ces quinze jours, vous devriez avoir un indicateur convenable de l'usage de chacun de vos documents imprimés.

Ajoutez cette information à la colonne «encore utile» de votre tableau. Une fois l'audit terminé, gardez-le et utilisez-le comme un outil d'audit permanent, pour garder trace de tous les nouveaux documents.

Attribuez-vous 10 points si plus de $50 \%$ de vos documents imprimés sont utilisés, 5 points si c'est moins de la moitié et 0 point si moins du quart est réellement utilisé.

\section{Créez et entretenez}

des supports de communication utiles et pertinents

Si vous avez réussi l'audit proposé ci-dessus, bravo! Vous êtes sur la bonne voie. Voilà quelques idées complémentaires. 
Gardez un œil sur les tendances aux bureaux d'accueil: rencontrez régulièrement les personnes chargées de l'accueil (une fois par mois suffit) pour déterminer quelle est la question récurrente du moment. Certains mois, vous serez tenté de créer un vademecum pour répondre sur-le-champ à la question; à d'autres occasions, vous attendrez de voir si la tendance se confirme plusieurs mois de suite avant de créer un nouveau document. Vous devrez faire preuve de discernement pour trouver le bon rythme de création de documents face aux questions fréquentes: gardez à l'esprit que vous devez vous appuyer sur les preuves tangibles d'un besoin avant de créer quoi que ce soit.

\section{Envisagez des alternatives en ligne ou de médiation directe}

On trouve souvent maintes traces d'un passé révolu sur les présentoirs: si votre audit a permis d'en dénicher, la solution est à portée de main. Certains supports ont été imprimés à une époque où cela avait du sens de présenter une nouvelle ressource en ligne ou sur CD-Rom sous forme de flyer, près de l'unique poste de consultation. Ce n'est plus le cas aujourd'hui: pourquoi ne pas proposer plutôt un screencast, une vidéo de présentation en ligne (qui présentent le même risque de péremption rapide et exigent un fort investissement côté mise à jour eux aussi) ou des ateliers bien ciblés?

\section{Impliquez les utilisateurs}

Demandez-leur leur avis: c'est le meilleur moyen de vous assurer une approche fondée sur les preuves en la matière. Quand vous avez une nouvelle idée, pourquoi ne pas la soumettre aux utilisateurs eux-mêmes en leur demandant, via le canal de votre choix, si le document que vous vous proposez de faire leur serait utile. Souvenez-vous que si vous vous contentez de demander l'opinion des utilisateurs ("que pensez-vous de?»), vous n'aurez en retour qu'une opinion (et ceux-ci ont tendance à être très polis lorsqu'on 
leur demande leur opinion): assurez-vous de bien cibler la question sur l'utilité du document et l'usage qu'ils en feraient.

\section{Utilisez vos personas}

La section 2.2 a montré l'intérêt des personas pour aider votre bibliothèque à cibler ses ressources et services sur les vrais utilisateurs. Si vous avez déjà créé vos personas, utilisez-les à chaque fois que vous envisagez de rédiger un nouveau support de communication et demandez-vous si l'une d'entre elles le trouverait utile. Si vous n'avez pas encore de persona, qu'attendez-vous donc pour en créer?

\subsection{Valoriser ses ressources}

\section{Difficulté *}

Si vous avez eu l'occasion de fréquenter un magasin de bricolage ces dernières années, vous avez sûrement remarqué que la promotion des produits in situ se fait beaucoup. On ne compte plus les études consacrées au comportement du consommateur dans les grandes surfaces, les analyses sur l'impact de l'emplacement des produits sur les linéaires en termes de ventes et les ouvrages entiers de marketing dédiés à la question de la valorisation des produits. L'ambition de cet autodiagnostic n'est pas d'en rendre compte in extenso, mais d'en condenser l'essentiel en tirant de l'expérience des grandes surfaces des leçons utiles et applicables en bibliothèque.

\section{Pourquoi c'est important}

Bien qu'il soit très simple de valoriser ses collections, bien des bibliothèques, notamment universitaires, mais parfois en lecture publique, ne s'en préoccupent pas.

C'est fort dommage, car la valorisation des collections est un excellent moyen de mettre en relation des lecteurs et des ouvrages 
qu'ils n'auraient, sans cela, jamais remarqués. Ce type de découvertes (le mot à la mode est «sérendipité») fait le charme de bien des bibliothèques et insuffle plus de vie aux collections. De fait, ce processus physique de découverte stimule plus l'imagination et l'envie que les suggestions en ligne, et devrait être considéré comme un des avantages concurrentiels des bibliothèques sur Amazon, et autres vendeurs de livres en ligne. Il y a pléthore de contenus intéressants dans les rayonnages de votre bibliothèque. Pourquoi ne pas trouver un, ou mieux, des endroits pour les mettre en lumière et en valeur?

Valoriser les collections de sa bibliothèque, c'est tout mettre en œuvre pour améliorer leur visibilité. Imaginez qu'il s'agit d'une librairie attrayante: tables dans les zones de forte fréquentation permettant de présenter des nouveautés, mise en avant des best-sellers, sélections thématiques, présentation d'une sélection d'ouvrages en bout d'étagères, titres sur présentoir en rayon pour mettre en évidence les belles premières de couverture. Ce sont des idées toutes simples que vous pouvez observer dans n'importe quelle librairie et, bien sûr, dans les bibliothèques qui savent y faire.

\section{À vous de jouer}

Les calculs sont simples pour cet autodiagnostic: soit vous faites en permanence des présentations de livres sur tables et en bout de rayonnages, soit vous ne le faites pas. Si vous le faites, marquez 20 points.

Bien sûr, valoriser des titres ne suffit pas. Vous voudrez aussi savoir si ce que vous mettez en évidence est emprunté ou non. Si tel n'est pas le cas, vous devrez revoir la manière dont vous sélectionnez et mettez en valeur les documents.

Que votre bibliothèque valorise ses collections depuis longtemps ou depuis peu, voici quelques idées pour mieux attirer l'attention sur vos collections. 
Ne jetez pas les jaquettes

Si vous commandez vos livres déjà tout équipés ou si vous les faites relier, prenez l'option « conserver la jaquette».

\section{Proposez des sélections thématiques}

Une table de nouveautés va de soi, mais vous devriez aussi penser à mettre en présentation, de manière très régulière, des thèmes d'intérêt local, d'actualité, des curiosités: les options possibles sont infinies et la seule limite est celle de votre imagination.

\section{Encouragez les prêts}

Avez-vous déjà remarqué que les supermarchés mettent les bonbons, les magazines de cuisine et la presse locale près des caisses? Les clients sont plus enclins à prendre un paquet de chewing-gum et 50 recettes pour réussir les fêtes avec Cyril L. quand ils font la queue pour payer que lorsqu'ils les voient en rayon. Les achats d'impulsion portent bien leur nom! Si vous mettez en valeur des livres à proximité des automates de prêt ou de la banque de prêt, vous devriez observer le même effet d'emprunt impulsif (sans sucre ajouté !)

\section{Les retours du jour}

La plupart des sites de vente en ligne attirent l'attention sur les achats des autres consommateurs pour vous encourager à faire de même. C'est la raison pour laquelle on voit souvent la mention «Ceux qui ont acheté ce produit ont également acheté...».

Pourquoi? La psychologie humaine est ainsi faite: nous aimons savoir ce que font les personnes qui nous ressemblent. Jouant sur ce constat, certaines bibliothèques mettent en évidence des ouvrages qui viennent juste d'être rendus, dans l'idée que les autres membres de la communauté pourraient être intéressés par 
ce que leurs voisins viennent de lire/regarder/écouter. Si vous pouvez extraire les données de circulation de votre SIGB, il vous est même possible d'afficher ces données sur des écrans dans votre bibliothèque. Un bon exemple de cette démarche est l'Awesome Box (Boîte à trouvailles) du Harvard Library Innovation Lab: lorsqu'un lecteur place un retour dans une Awesome Box non seulement ce document est mis en avant, mais encore un tweet est envoyé et l'ouvrage ajouté à une liste des "Recently Awesome» (<http://librarylab.law.harvard.edu/projects/awesome-box >).

\subsection{Les services de la bibliothèque apportent des solutions aux problèmes des utilisateurs}

\section{Pourquoi c'est important}

\section{Difficulté ***}

Les services rendus par la bibliothèque sont sa raison d'être. Sans services, pas de bibliothèques. Il paraît donc logique qu'elles soient conscientes de leur importance dans la vie des habitants.

Depuis le début, ce livre insiste sur le fait que les bibliothèques doivent s'efforcer d'être utiles, utilisables et désirables et sur la façon d'améliorer leur utilisabilité et leur attrait.

Ces progrès amélioreront certainement l'expérience des membres de la communauté que vous desservez, mais vous ne pouvez pas en rester là. La section 7.4 du chapitre 7 souligne qu'il ne suffit pas d'un design réussi pour que les gens utilisent un site web de bibliothèque s'il est truffé de contenus sans intérêt. Le même principe s'applique à nos bibliothèques tout entières. Une bibliothèque peut avoir un magnifique bâtiment, être pleine de bibliothécaires sympathiques et dotée d'une signalétique de premier ordre, si ses services ne sont pas pertinents, personne ne l'utilisera. 
C'est le dernier point de ce livre: il figure à la fin parce que, par bien des aspects, il s'agit du point le plus important. Il vous met au défi de repenser vos services et d'explorer de nouvelles possibilités.

\section{Qu'est-ce qu'un problème?}

Réfléchir à ce qu'on entend par problème en vaut la peine: en un sens, un problème est une énigme qui doit être résolue. Prenez les problèmes de maths: ce sont simplement des énigmes en attente de solutions.

La notion de "problème vu comme une énigme» peut être utilisée pour décrire autre chose que des équations. Un problème peut servir à analyser le comportement de quelqu'un.

Voilà un exemple: Marceline commande un thé au comptoir. Pourquoi?

Ses motivations peuvent être variées:

$\odot$ elle a soif et trouve le thé rafraîchissant;

$\odot$ elle aime le goût du thé;

$\odot$ elle doit commander une boisson quelconque pour pouvoir utiliser le wi-fi.

Le simple fait de commander une tasse de thé peut donc répondre à des motivations très différentes. Utiliser le mot problème dans le sens de «énigme à résoudre» est un mode de pensée à part entière qui permet de mieux réfléchir à ce dont les personnes ont besoin et à la manière dont les bibliothèques peuvent y répondre.

Un sens plus littéral, et plus commun, du mot problème implique un enjeu pénible, quelque chose qui doit être réglé. Par exemple, le problème du réchauffement climatique ou des sans-abri. Il n'y a pas grand-chose à ajouter sur cette acception courante du mot problème. 
Les bibliothèques doivent résoudre des problèmes, dans les deux sens qui viennent d'être discutés:

$\odot$ les bibliothèques rendent de meilleurs services si elles pensent aux besoins des membres de la communauté qu'elles desservent comme à des énigmes à résoudre;

$\odot$ les bibliothèques doivent aider à résoudre les «vrais » problèmes de leur communauté.

\section{À vous de jouer}

Cet autodiagnostic est assez complexe. Il est pourtant suffisamment important pour que vous y consacriez autant de temps et d'efforts que nécessaire pour évaluer votre situation actuelle. Pour cela, utilisez en particulier deux techniques de recherche sur les usagers: les entretiens et les sondes culturelles (entretiens ethnographiques allégés).

\section{Entretiens avec des utilisateurs}

Discutez avec cinq à huit membres de la communauté que vous desservez. Recrutez des personnes qui utilisent déjà la bibliothèque, mais pensez également à y inclure quelques-unes qui ne l'utilisent pas. Posez des questions ouvertes sur leurs vies, leurs besoins, leurs buts et leurs motivations. Si vous pouvez l'éviter, ne posez pas de question sur la bibliothèque elle-même: en parler pourrait influencer leur propos et leur regard. Gardez la conversation ouverte et générale: le but de ces entretiens est de collecter des instantanés de la vie des gens de manière à vous permettre d'imaginer quand et où la bibliothèque peut les aider à résoudre leurs problèmes.

\section{Sondes culturelles}

Recrutez cinq à huit autres membres de la communauté que vous desservez (une nouvelle fois sans vous cantonner aux inscrits). 
Fournissez à chaque personne un appareil photo et un carnet, sous la forme d'une tablette, par exemple. Demandez-leur de prendre des photos et d'utiliser les journaux pour raconter leur quotidien. Menez l'exercice sur quelques semaines, de manière à collecter beaucoup de données. Donnez des instructions précises aux participants sur ce qu'ils doivent vous rendre à la fin de l'expérience (par exemple, au moins une photo par jour et une entrée dans le carnet tous les deux jours) et des idées de ce qu'ils peuvent y mettre. Comme pour les entretiens, le but de cet exercice est de collecter quelques instantanés d'autres vies que la vôtre.

\section{Comment estimer cela?}

Il n'existe pas de moyen simple pour évaluer la capacité d'une bibliothèque à résoudre les problèmes de la communauté qu'elle dessert.

Après avoir fait les deux exercices d'exploration ci-dessus, vous comprendrez mieux ce que les problèmes de votre communauté ont de spécifique: c'est un bon début. Pour les besoins de cet exercice, donnez-vous 30 points si vous estimez que votre bibliothèque fait déjà du bon travail pour les résoudre avec les services existants, 15 points si vous pensez que vous faites un travail qui va dans la bonne direction, et 0 point si vous sentez que votre marge de progression est encore importante.

\section{Améliorez les services en résolvant vraiment les problèmes}

Si votre score est mauvais, ne désespérez pas. Il y a plein d'idées pour améliorer vos services et faire en sorte qu'ils résolvent activement les problèmes de votre communauté. Comment y arriver?

Penser les services comme des réponses aux problèmes rencontrés par les personnes vous place dans un cadre conceptuel centré sur elles. Il ne s'agit pas seulement d'avoir un point de vue 
centré sur le fait «d'aider», mais aussi d'examiner leurs aspirations et leurs motivations fondamentales. Mieux les connaître permet de créer des solutions intéressantes pour répondre à leurs besoins et construire des relations fortes.

Prenez une pratique courante en bibliothèque: utiliser un ordinateur pour aller sur Internet. Quel est le problème que la bibliothèque doit résoudre?

Prenons le cas pratique suivant: Marcel veut avoir accès à Internet. Qu'est-ce qui est réellement en jeu pour Marcel? Est-il en recherche d'un emploi après avoir perdu le sien? Se distrait-il sur YouTube parce qu'il s'ennuie? Essaie-t-il de terminer un mémoire d'étude? Veut-il apprendre des techniques nouvelles en lien avec son dada du moment? Son objectif immédiat est d'aller sur Internet, mais ses motivations nous entraînent beaucoup plus loin.

Supposez qu'il veuille apprendre des techniques nouvelles en lien avec son activité préférée: allez plus loin. Pourquoi veut-il accroître son savoir-faire? Quel problème cela lui permet-il de résoudre? Peut-être veut-il être meilleur que ses copains? Peut-être veut-il en savoir plus pour pouvoir enseigner quelque chose à d'autres?

Tous les autres services de la bibliothèque peuvent être analysés à travers ce prisme: les usagers utilisent les collections, participent aux animations, utilisent les espaces. Il faudrait se pencher avec de l'empathie et une attention sincère sur toutes ces intentions qui font appel aux services de la bibliothèque, pour mieux comprendre leurs motivations. Mieux vous les connaîtrez et plus vous les comprendrez, plus grand sera le rôle des bibliothèques. Et plus le rôle de la bibliothèque dans la vie de la population est grand, plus grande est sa valeur. Avoir des services qui créent de la valeur est la meilleure défense possible des bibliothèques. C'est la clé qui leur permet de devenir essentielles à la vie de la cité.

Ne vous contentez pas de réfléchir à la manière dont votre bibliothèque peut fournir des animations et des services dont les 
membres de votre communauté pourraient avoir besoin. Essayez de voir si vos animations et vos services proposent des solutions aux problèmes qu'ils rencontrent. Souvenez-vous: ils ne viennent pas à la bibliothèque pour lire des livres ou utiliser le wi-fi. Ils viennent pour apprendre, progresser, s'amuser ou établir des liens avec les autres. Lire des livres et aller sur Internet sont seulement deux moyens parmi d'autres pour les y aider. Concevoir des services qui aident les individus à accomplir leurs projets permettra à votre bibliothèque d'évoluer et d'aller plus loin que son rôle traditionnel de hangar à contenus.

\section{Les bibliothèques règlent les problèmes des citoyens}

Cette idée n'est ni nouvelle ni radicale. Jusqu'aux années 1990, l'information était une ressource rare et les bibliothèques permettaient d'y donner accès: tout d'abord en mettant à disposition des documents imprimés, puis en fournissant un accès à Internet. Par la suite, l'information est devenue moins rare et l'accès à Internet s'est largement diffusé dans la société. Depuis lors, le rôle central de la bibliothèque au sein de la cité n'est plus de résoudre le problème de l'accès aux informations.

Et pourtant, bien des bibliothèques s'accrochent à l'idée que ce qui fait leur valeur singulière au sein de leur communauté est de fournir un accès à du contenu, et rien que du contenu, que ce dernier soit éditorial, patrimonial, professionnel ou universitaire. Il n'est pas question ici de nier l'intérêt des contenus. Un des rôles des bibliothèques reste bien d'être des pourvoyeuses de contenus. Simplement, les bibliothèques peuvent aller beaucoup plus loin que ce seul rôle traditionnel pour répondre aux besoins de leurs utilisateurs et les aider à résoudre leurs problèmes.

Voici quelques exemples de bibliothèques qui ont su nouer des partenariats avec d'autres services destinés aux citoyens, ou qui ont étendu leurs missions au-delà de leur périmètre traditionnel. 


\section{Baltimarket}

La Enoch Pratt Free Library, située dans un quartier très pauvre de Baltimore, collabore avec d'autres organisations pour proposer des paniers de fruits et légumes là où les habitants en manquent. Elle a joué un rôle majeur au moment des émeutes de 2014 pour maintenir un lien social dans la cité et fournir aux familles des produits de première nécessité et un lieu sûr.

\section{Une infirmière intégrée}

Toutes les bibliothèques publiques ont un rayon «Santé et bienêtre». La bibliothèque publique de PIMA County (<http://www. library.pima.gov $>$ ) a franchi une étape supplémentaire pour fournir ce type d'informations et a engagé, à la bibliothèque même, une infirmière spécialisée en santé publique, qui s'occupe d'animations, est disponible pour répondre aux questions et faire des recherches avec les gens si nécessaire. Combiner expertise professionnelle et connaissance des bases de données ouvre des champs très larges pour aider la population en matière de santé.

\section{LibraryYOU}

Ce projet de la bibliothèque publique d'Escondido ( $<$ https://libraryyou.escondido.org/ >) ouvre de nouveaux horizons sur les collections de la bibliothèque. La bibliothèque aide sa communauté à créer et publier des vidéos et podcast pour «collecter et partager la mémoire locale».

\section{Aide sociale intégrée}

Quand votre bibliothèque est située dans une grande ville, sa localisation a une importance majeure: certaines bibliothèques de quartier ont à gérer des problèmes sociaux comme celui des sans-abri, des toxicomanes ou d'autres formes de marginalisation. Certaines bibliothèques, comme celle de San Francisco, 
répondent à ces problèmes en engageant un travailleur social pour les bibliothèques de certains quartiers, à même d'aider les gens confrontés à ces difficultés aiguës.

On peut s'opposer à ce que les bibliothèques s'investissent dans de tels projets, et y voir un signe d'abandon de leurs missions premières. On peut aussi considérer qu'une vision étroite des missions des bibliothèques est une stratégie à courte vue. Dans la mesure où les individus ont des moyens de plus en plus variés pour accéder à des contenus, il est plus important que jamais pour les bibliothèques de trouver de nouvelles manières d'aider les communautés qu'elles desservent. Dit plus simplement, si vous voulez que vos concitoyens accordent de la valeur à votre bibliothèque, elle doit impérativement répondre à un besoin auquel aucune autre organisation ne répond.

\subsection{Plus de lectures}

Anderson R., "What Patron-Driven Acquisition (PDA) Does and Doesn't Mean:An FAQ”, The Scholarly Kitchen, 31mai2011. [En ligne] < http://scholarlykitchen.sspnet.org/2011/05/31/whatpatron-driven-acquisition-pda-does-and-doesnt-mean-an$\mathrm{faq} />$.

Working Together Project (Canada). Community-led Libraries Toolkit, Toronto, 2008. [En ligne] < http://www.librariesincommunities.ca/resources/Community-Led_Libraries_Toolkit.pdf $>$. 


\section{Chapitre 9. Conclusion: philosophie, processus et culture}

Il y avait beaucoup à dire sur les différentes facettes des bibliothèques dans tout ce que vous venez de lire: vous avez maintenant plein d'idées et de modes d'emploi pour effectuer des changements centrés sur les utilisateurs dans votre bibliothèque. Ce dernier chapitre propose une mise en contexte de ces changements: comment penser de manière globale l'ensemble des points de contact entre votre bibliothèque et ses utilisateurs? Une méthodologie, une démarche fondée sur le design de service (service design) peut-elle être utile pour appréhender ces défis? Quelle culture organisationnelle mettre en place pour être vraiment centré sur l'utilisateur?

\subsection{Penser la bibliothèque comme un tout}

Si vous avez fait tous les autodiagnostics proposés dans ce livre, vous connaissez vos points forts, vous savez où et quand votre bibliothèque favorise de bonnes expériences pour ses utilisateurs. Vous savez aussi, à présent, quels sont les points de contact qui doivent encore être améliorés. Vous avez sans doute envie de vous y mettre tout de suite et de faire ce qui semble à votre portée. Parfait.

Les différents points de contact ne sont pas «hors sol». Avant de commencer à les prendre un à un, reconsidérez les besoins des publics.

Même si vous avez analysé et évalué les points de contact "par grandes familles», pensez que les personnes qui ont affaire à la 
bibliothèque sont la plupart du temps confrontées à plusieurs «familles» de points de contact.

Votre objectif est d'évaluer et d'améliorer votre score d'utilisabilité total et par voie de conséquence, l'expérience globale des utilisateurs: il est donc très important d'avoir une vue panoramique et de penser les points de contact en situation, sans les prendre un par un.

Pour cela, réfléchissez à ce que les utilisateurs essaient de faire dans votre bibliothèque: vous souvenez-vous de la discussion sur les missions critiques du site web de la bibliothèque à la section 7.2? Demandez-vous ce que les personnes cherchent à faire lorsqu'elles utilisent la bibliothèque. Voilà quelques exemples qui peuvent vous aider à dresser votre propre liste:

○ retirer un document réservé;

$\odot$ imprimer un devoir à rendre en classe;

$\odot$ utiliser une salle de travail de groupe;

$\odot$ assister à une animation ou un atelier organisés par la bibliothèque.

\section{Cartes d'itinéraires}

Pour penser ces opérations de manière globale, modélisez chacune des étapes par lesquelles doit passer quelqu'un qui utilise votre service. En voici un exemple:

Venir chercher un document réservé:

1. Faire une réservation en ligne (présence en ligne);

2. Recevoir une notification par e-mail (présence en ligne);

3. Faire le trajet jusqu’à la bibliothèque;

4. Se garer (lieu); 
5. Entrer dans le bâtiment (lieu);

6. Être accueilli par un bibliothécaire (service);

7. Trouver où sont rangées les réservations (signalétique);

8. Se rendre au rayon «documents réservés» (lieu);

9. Trouver le livre réservé sur le rayonnage (mobilier et signalétique);

10 Marcher jusqu'au point de prêt/retour (lieu);

11. Interagir avec un bibliothécaire (service) ou un automate de prêt (matériel);

12. Sortir du bâtiment (lieu).

Cette liste montre comment s'articulent les différents points de contact et met en évidence leur interconnexion. Ce type de liste peut être utile pour évaluer l'expérience globale de l'utilisateur pour chaque tâche à réaliser.

Si une tâche à accomplir se décompose en une série d'opérations qui nécessitent d'être améliorées, les utilisateurs de la bibliothèque doivent avoir bien des difficultés à faire ce qu'ils ont à y faire. Si l'ensemble des étapes à franchir ne présente qu'un seul point noir, ces utilisateurs ont sans doute une expérience globale convenable.

Ce type de carte d'itinéraires peut vous permettre de prioriser vos efforts d'amélioration.

Comparez plusieurs cartographies. Certaines comportent-elles plus de points de friction que d'autres? Commencez par elles. Laissez de côté celles qui concernent seulement des points mineurs, jusqu'à ce que vous ayez réglé de manière satisfaisante les gros problèmes. Souvenez-vous: ces points de contact sont utilisés par beaucoup de monde: les améliorer aura un impact important. 
Vous pouvez pousser plus loin votre cartographie et en faire une représentation graphique: cela peut vous donner des idées concrètes de la fluidité de l'expérience globale. Vous pouvez aussi aller au plus simple et ne pas passer par la phase graphique. Une simple liste peut déjà être très efficace.

\section{Des cartes d'itinéraire pour rénover et innover}

Ces cartes d'itinéraires peuvent vous aider à résoudre les points noirs, mais aussi à aller plus loin. Elles peuvent même vous aider à revoir vos services de fond en comble, en examinant bien plus de possibilités et en allant débusquer le diable de l'immobilisme là où il se tapit: dans les détails.

Pour chaque point de contact, posez-vous les questions suivantes:

$\odot$ présupposés sur les objectifs des usagers: quel est leur but, que cherchent-ils à faire?

$\odot$ présupposés sur le point de contact lui-même: pourquoi est-il agencé ainsi?

$\odot$ l'objectif du contact: le but pourrait-il être atteint d'une autre manière?

$\odot$ pourquoi y a-t-il besoin de ce point de contact précis? Le parcours pourrait-il être simplifié ou rendu plus efficace sans lui? Qu'est-ce qui pourrait le remplacer?

$\odot$ comment ce point de contact est-il articulé avec celui qui le précède et celui qui le suit? Passe-t-on facilement d'une étape à la suivante?

Cette réflexion de fond peut aller encore plus loin si vous reformulez la liste des "opérations essentielles »: vous avez remarqué que l'exemple proposé était très centré sur la bibliothèque et ne listait 
que les comportements des individus en relation avec la bibliothèque, sans envisager ce qu'ils cherchent vraiment à accomplir.

Si on reformule les faits en les inscrivant dans une perspective plus large, cela pourrait donner quelque chose comme:

$\odot$ lire un document à l'extérieur de la bibliothèque;

$\odot$ avoir une bonne note;

$\odot$ lire ou étudier dans un endroit calme;

$\odot$ se divertir ou apprendre quelque chose au sein d'un groupe;

$\odot$ trouver un livre en particulier.

Envisager ainsi ce que font les utilisateurs dans la bibliothèque peut vous permettre d'explorer plusieurs manières de les aider à atteindre leurs buts.

Par exemple, pour la première tâche, lire un document à l'extérieur de la bibliothèque, au lieu de vous concentrer sur la réservation et le retrait du document, vous pouvez réfléchir et imaginer d'autres services comme la fourniture de documents à domicile ou des points relais dédiés au retrait des documents au plus près de la communauté à desservir.

Discuter des motivations profondes des utilisateurs de bibliothèque et faire dans la foulée un brainstorming classique autorisant toutes sortes d'idées farfelues, sans autocensure, peut faire naître de nouvelles idées et devenir un catalyseur de changement. Les idées les plus prometteuses pourront être testées et mises en place en suivant la démarche de design.

\subsection{La démarche de design}

\section{Concevoir votre bibliothèque en termes de design}

Comme cela a déjà été dit en introduction, vous êtes le M. Jourdain du design en bibliothèque. Que vous le vouliez ou non, que vous 
en soyez conscient ou pas, à chaque fois que vous prenez une décision sur l'apparence ou le fonctionnement de quelque chose dans votre bibliothèque, vous faites du design. Vu sous cet angle, dans une acception très large, le design consiste à arranger des choses dans un but déterminé.

Un des objectifs de ce livre est d'aider les bibliothécaires à prendre des décisions de design, à concevoir les choses de manière délibérée plutôt que de les faire par défaut.

Inconsciemment, il y a bien des choses faites par défaut: utiliser une police par défaut, aménager un espace par défaut, ou avoir un mode de pensée par défaut, dépourvu de recul ou continuer à faire ce qu'on a toujours fait. Concevoir un service "par défaut» n’a jamais conduit à des résultats optimaux pour les utilisateurs.

Lire ce livre, c'est faire un premier pas dans la bonne direction, et commencer à concevoir les choses dans un but délibéré: au fil des chapitres, vous avez vu l'impact des différentes composantes de la bibliothèque sur l'expérience qu'en ont les utilisateurs, découvert le processus d'optimisation des points de contact et travaillé sur une nouvelle manière de réfléchir à la bibliothèque.

Vous devriez être en mesure de prendre de meilleures décisions après l'avoir lu, mais ce pourrait n'être qu'un début. Vous pouvez être encore plus performant si vous concevez le design non comme un acte isolé, mais comme une démarche continue.

\section{La démarche de design}

Il existe de nombreuses méthodes de design: elles ont toutes en commun certains éléments. En gros, elles s'articulent autour de quatre axes principaux: observer, faire des prototypes, tester, implémenter. 


\section{Observez}

Au cours de cette étape, votre but est d'en apprendre davantage sur le problème que vous cherchez à résoudre. Vous commencez à apprendre dès que vous essayez de formuler clairement le problème. Voici quelques exemples de formulation:

$\odot$ il y a des files d'attente trop longues pour emprunter des livres;

$\odot$ le taux de fréquentation des animations est bas;

$\odot$ le personnel à l'accueil est trop occupé pour répondre à toutes les questions reçues.

Passez du temps à peaufiner la formulation. La plupart du temps, c'est utile pour se recentrer sur les utilisateurs. Si on reprend le premier problème:

$\odot$ les usagers s'impatientent lorsqu'ils font la queue pour emprunter des livres;

$\odot$ en soirée, le temps d'attente moyen avant d'emprunter est pénible.

La manière dont vous formulez la question a un impact sur le type de solution que vous proposerez. La première formule peut amener à une solution permettant de distraire les gens lorsqu'ils font la queue; la seconde induire plutôt une solution du type «augmenter les effectifs» ou «installer des automates de prêt». Il arrive que dans la phase de définition d'un problème, on en arrive à des conclusions erronées. Si cela devait se produire, vous vous retrouveriez alors à tenter de résoudre le mauvais problème: assurez-vous donc d'avoir posé la bonne question en amont!

Une fois que vous savez quel est le problème à résoudre, vous aurez à poser de nombreuses questions et à vous plonger dans les détails: reportez-vous au paragraphe «Des cartes d'itinéraires pour innover». Les enquêtes contextuelles et les interviews d'usagers sont de bons outils complémentaires. 


\section{Faites des prototypes}

À ce stade, vous imaginerez un certain nombre de solutions potentielles au problème. Pour produire des idées, divisez le problème en étapes distinctes.

Revenons au cas de Marceline, la buveuse de thé rencontrée en 8.5, qui voulait boire du thé et n'en avait pas sous la main.

Si elle avait une tasse de thé devant elle, tout serait simple. Comme ce n'est pas le cas, le problème est un peu plus compliqué. Plusieurs solutions s'offrent à elles:

$\odot$ préparer du thé;

$\odot$ aller au café et commander une tasse;

$\odot$ demander à un ami d'en rapporter.

Chacune de ces solutions peut être décomposée à son tour. Pour préparer du thé, vous avez besoin:

$\odot$ d'une tasse;

$\odot$ d'eau chaude;

$\odot$ d'un sachet de thé.

Plusieurs moyens possibles de répondre à ces trois besoins:

1) J'ai besoin d'une tasse:

$\odot$ j'en prends une dans le placard;

$\odot$ j’utilise le verre que j’ai déjà;

2) J'ai besoin d'eau chaude:

$\odot$ je mets de l'eau à bouillir dans la bouilloire électrique;

$\odot$ j'utilise l'eau très chaude du robinet;

3) J'ai besoin d'un sachet de thé:

$\odot$ j'en prends dans le placard;

$\odot$ je remplis une boule à thé de feuilles en vrac. 
Ainsi décomposé, se faire une tasse de thé paraît toute une affaire, et on s'étonnerait presque d'y arriver! À vrai dire, tout le monde enchaîne jour après jour ce genre de microdécisions sans même y penser.

Et pourtant, prendre le temps d'analyser pas à pas un comportement peut donner des résultats intéressants. Un vendeur de thé voudrait sans doute examiner tous ces problèmes afin de chercher à améliorer l'expérience quotidienne du buveur de thé. Décomposer les comportements des utilisateurs de la bibliothèque jusqu'à ce niveau de détail vous donnera un tableau complet et vous permettra de passer outre certaines idées préconçues et partant, d'arriver à de vraies innovations.

Une bonne manière de créer des prototypes est de commencer par un brainstorming classique. Le groupe peut proposer toutes sortes de solutions et en parler. Les idées farfelues et irréalistes doivent être encouragées et décrites comme les autres. Elles peuvent, par contraste, faire apparaître l'intérêt d'alternatives. Les meilleures idées peuvent être combinées, et le groupe peut décider de celles qui méritent d’être creusées.

Une fois un accord trouvé sur des solutions potentielles, vous aurez à vérifier que vos intuitions sont les bonnes en testant ces idées sur le terrain.

\section{Testez}

Pour tester vos prototypes, vous aurez à les présenter à tout le personnel et à évaluer s'ils fonctionnent bien ou non. Si vous testez une nouvelle manière de valoriser des documents, prévoyez un temps pour mesurer le taux de circulation. Si vous testez une nouvelle arborescence de votre site web, vous conduirez des tests d'utilisabilité.

En testant vos prototypes, vous saurez si vous êtes ou non sur la bonne piste. Si votre prototype n'apporte pas de solution, ne 
vous en faites pas! Pensez l'ensemble de la démarche comme un moyen de multiplier les cycles d'essai-erreur, et d'échouer rapidement, sans avoir investi trop d'énergie. Comprendre l'échec comme une occasion de recommencer plus intelligemment est capital. Il est préférable de trouver ce qui marche et ne marche pas en testant un scénario plutôt que de le découvrir à l'issue d'une implémentation complète. Apprenez, en regardant vos prototypes, à repérer ce qui fonctionne bien et ce qui ne fonctionne pas. Comme pour les idées, vous pouvez combiner les différents éléments de prototypes qui fonctionnent. Après avoir évalué les résultats de vos tests, vous parviendrez à la meilleure solution.

\section{Implémentez}

Une fois que vous avez appris tout ce qu'il y avait à apprendre en testant vos prototypes les plus prometteurs, il est temps de mettre en action les meilleures idées. Ce n'est pas toujours aussi facile qu'il y paraît. Selon l'ampleur des changements projetés, il vous faudra planifier bien des choses: la formation du personnel, d'éventuels travaux, etc. L'important est que tout le monde ait le même degré d'information et que les services travaillent ensemble: ce sera d'autant plus facile que vous aurez associé les personnes concernées tout au long de la démarche, et depuis le début.

\section{Tout est objet de design}

Cette démarche peut s'appliquer dans n'importe quel contexte. Par exemple, les auteurs du livre de recettes Cook's Illustrated utilisent ce procédé pour écrire leurs recettes. Ils commencent par observer un problème (par exemple, celui de la salade de thon trop souvent aqueuse) et cherchent toutes sortes d'idées pour le pallier (laisser égoutter le thon dans un torchon? le 
centrifuger?), puis ils essaient de faire le plat en appliquant les méthodes proposées. La recette passe par maintes itérations qui permettent de vérifier ce qui marche et ce qui ne marche pas, avant de publier la version finale.

Voilà: les bibliothèques et tout ce qu'elles contiennent ou font peuvent être un objet de design. Une telle méthode libère de bien des freins: chacun pouvant se positionner comme un designer à son niveau d'action et sur son terrain, il n'y a plus à attendre un éclair de génie, un leader charismatique ou des moyens extraordinaires pour améliorer les bibliothèques. Vous disposez d'une méthode prête à l'emploi pour innover et améliorer les choses en équipe. Il ne vous reste plus qu'à fermer ce livre et vous y mettre.

Tout concevoir selon la démarche de design? Tout peut être objet de design: cela ne veut pas dire que chaque petite décision doit passer à travers toutes les étapes du processus de design. Vous n'arriverez jamais à rien si vous avez à interroger des usagers à chaque fois que vous concevez un nouveau marquepage. Cela étant dit, les marque-pages gagneront à être relus. En fin de compte, vous aurez juste besoin d'un peu de bon sens pour estimer le temps qu'il est possible de consacrer à la conception, selon une démarche de design d'un produit ou service donné.

\subsection{La culture de votre organisation}

Si vous êtes arrivé au bout de ce livre, c'est que vous êtes convaincu de la validité et de la viabilité du design de l'expérience utilisateur, de l'UX comme cadre conceptuel susceptible d'amener des changements positifs en bibliothèque. Avant que vous ne partiez tête baissée, voici quelques éclairages sur les 
enjeux de ces mises en place en termes d'organisation, et sur les moyens dont vous disposez pour relever le défi.

L'idée de base est la suivante: vous ne serez en mesure de mettre en œuvre les idées de ce livre que si vous avez la bonne culture dans votre organisation. La bonne culture? Oui, une culture qui place l'utilisateur au premier plan, à tous les niveaux, à commencer par l'adhésion pleine et entière de l'équipe de direction jusqu'à celle des agents les plus proches du terrain. Comment développer une telle culture? Voici quelques pistes.

\section{Développer de manière collective une vision et des valeurs communes}

On a vu en 5.1 l'importance d'avoir une philosophie des services qui implique tous les membres du personnel. Si les valeurs du service servent de fil directeur pour les professionnels à l'accueil, la vision et les missions de l'établissement doivent nourrir tout ce que vous faites. Les missions donnent un cadre, la vision une aspiration commune et un but à votre organisation. Assurez-vous de construire ces formulations avec les agents de la bibliothèque afin d'obtenir leur adhésion, et veillez à ce que les membres de la communauté que vous desservez y soient associés.

Si vous avez déjà une mission et une vision, c'est peut-être le bon moment pour les revoir collectivement, et pour vous assurer qu'elles visent bien la recherche de la meilleure expérience possible pour l'utilisateur.

\section{N'ayez pas peur de prêcher la bonne parole}

Amanda Etches et Aaron Schmidt racontent qu'une de leurs histoires préférées (autour de la refonte d'un site web) a pour protagoniste un bibliothécaire passionné par le Design UX. Ce dernier a mis en place une équipe projet et a commencé par 
distribuer à chacun, lors de la première réunion, un exemplaire de Don't Make Me Think (Je ne veux PAS chercher) de Steve Krug. Pourquoi était-ce une si bonne idée? Si vous avez lu le livre de Steve Krug, vous savez déjà que son approche, simple et pleine de bon sens, place l'utilisateur au centre de toutes les décisions de développement web. En donnant à chacun un exemplaire du livre, ce bibliothécaire s'assurait que chacun mettrait de côté ses propres préférences en termes de refonte du site web et partagerait le même état d'esprit et la même démarche pour développer un site vraiment orienté vers l'utilisateur.

Cet exemple illustre bien l'intérêt de promouvoir activement les bonnes idées et un certain état d'esprit. Il serait naïf de supposer que vous travaillez dans une organisation où chacun des membres du personnel place l'intérêt de l'utilisateur avant tout le reste. En fait, il est certain que personne ne travaille ainsi, non parce que vous êtes dans une mauvaise bibliothèque, mais parce que tout le monde est pris dans sa routine quotidienne faite de tâches répétitives, de dates limites, de petits problèmes à résoudre au quotidien et est prompt à oublier que la raison d'être d'une bibliothèque est d'améliorer la vie des gens. Tout le monde a besoin, à un moment donné, de se voir rappeler cette évidence.

N'ayez pas peur, si vous êtes convaincu, de partager votre intérêt pour l'UX. S'il vous arrive de tomber sur un article intéressant, un billet ou un livre sur le Design UX enthousiasmant, parlezen autour de vous. Si vous entendez parler d'une conférence sur le sujet, essayez d'organiser une présentation dans votre bibliothèque. Si vous pouvez traduire des textes en français, sous-titrer des vidéos ou partager des retours d'expérience, faites-le. Invitez des collègues à observer des tests d'utilisabilité, partagez vos documents supports.

Le meilleur moyen d'associer vos collègues au service d'une vision UX, c'est de leur permettre à leur tour d'intérioriser cette 
manière de penser en mettant à leur disposition ce qui vous a permis à vous, la première fois, d'être enthousiasmé par ces idées-là.

\section{Militez pour développer des compétences dédiées à l'uX}

Que vous soyez ou non dans l'équipe de direction de votre bibliothèque (ce qui vous donnerait plus de poids pour faire évoluer les profils et missions des collègues), militez pour avoir un bibliothécaire ou deux qui travaillent particulièrement sur les questions liées à l'expérience vécue par les utilisateurs. Avoir une personne dédiée, ou mieux, une petite équipe, est idéal, parce que cette personne ou cette équipe peuvent prendre le temps de vraiment superviser tous les aspects de l'expérience des utilisateurs dans votre bibliothèque.

Si aucun poste ne peut être créé ou redéployé à temps plein, vous pouvez essayer de mettre en place un groupe transversal qui travaille à évaluer et améliorer l'utilisabilité globale dans la bibliothèque. Même si un temps plein dédié est préférable (participer à un groupe transversal vient souvent s'ajouter à une pile d'autres responsabilités amplement suffisantes pour occuper un temps plein), un groupe transversal est mieux que rien, surtout pour initier une démarche d'amélioration de l'utilisabilité. Essayez de rassembler quelques personnes qui ont un réel intérêt pour ces questions et partagent cette façon de voir, donnez-leur des outils pratiques et déléguez-leur du pouvoir pour mener des changements concrets à leur échelle.

Que vous réussissiez ou non à mettre en place un poste dédié, une équipe ou un groupe de travail, faites en sorte que quelqu'un s'occupe d'UX: la meilleure idée du monde ne décollera pas si elle n'est pas portée par l'enthousiasme de quelqu'un de convaincu et prêt à la défendre. 


\section{Développez des compétences en termes de connaissance des usages}

Si vous avez eu l'occasion de mener des tests d'utilisabilité, vous savez combien il est éclairant de voir quelqu'un tâtonner sur votre site web, pour faire quelque chose qui vous paraît, à vous, évident. C'est pourquoi vous avez tout à gagner à associer des membres du personnel, aussi bien parmi les équipes d'accueil que chez les cadres, pour participer aux opérations de recherche sur les usages. Quiconque a participé à une étude d'observation dans le hall d'accueil de la bibliothèque et été témoin des difficultés rencontrées par les nouveaux arrivants pour trouver leurs marques ne peut qu'être convaincu de la validité des principes et méthodes de l'UX. Si vous arrivez à impliquer ces personnes, vous serez sans doute témoin de ces moments de prise de conscience. Participer aux travaux de terrain est la meilleure des formations.

\section{L'UX n'est pas un frein à l'innovation}

Une critique fréquente contre la méthode de l'UX et ses principes est qu'il ne s'agit pas d'une stratégie globale d'innovation et que se contenter de réagir à des observations de terrain fondées sur ce qui existe déjà empêche une bibliothèque d'innover. Cette critique vous sera certainement opposée: quel que soit votre enthousiasme, restez pondéré et expliquez calmement qu'une telle idée repose sur une méconnaissance fondamentale de ce qu'est l'UX. Si l'UX consistait à demander aux utilisateurs ce qui pourrait leur faciliter la vie, il est clair qu'on n'arriverait ainsi à aucune innovation. Vos opposants vous citeront certainement la formule légendaire (et apocryphe) attribuée à Henri Ford: «Si j'avais demandé aux gens ce qu'ils voulaient, ils m'auraient répondu des chevaux plus rapides.» 
Dans la mesure où l'UX a pour but de comprendre les comportements des usagers (ce qu'ils font en réalité et non ce qu'ils pensent et déclarent faire), il n'y a rien qui vous empêche de rêver des solutions innovantes aux problèmes de vos utilisateurs, auxquelles ils n'auraient jamais pensé avant que vous les leur proposiez.

\section{Les contraintes administratives ou liées à l'organisation n'ont pas à être éliminées}

Lors d'ateliers sur la conception UX des sites web de bibliothèque, l'une des questions le plus souvent posées concerne la place du règlement de la bibliothèque sur le site web.

La question commence souvent par: "La direction m'oblige à mettre le règlement intérieur de la bibliothèque sur le site web. Je sais bien que nos utilisateurs n'en ont rien à faire. Que puisje faire?»

Même si cette question n'est pas un problème pour vous, vous êtes sans doute confronté à des tensions comparables entre ce qui est bon pour vos utilisateurs et ce qui est bon pour la bibliothèque.

Dans son livre fondateur, Elements of User Experience, Jesse James Garrett, l'un des parrains du Design UX, note que les besoins des utilisateurs ne sont qu'un des éléments du développement du produit final, les autres étant les contraintes financières (ou les contraintes de la bibliothèque), les nécessités techniques et les exigences de contenu.

En résumé, il ne faut pas être naïf et croire que les besoins de l'utilisateur sont le seul élément à prendre en compte lorsque l'on conçoit des choses (sites web, espaces, interfaces, guichets de service, quoi que ce soit d'autre) pour la bibliothèque; bien d'autres éléments doivent entrer en considération.

Donc, que répondre au pauvre bibliothécaire qui doit se débrouiller pour caser un règlement intérieur sur le site web? En gros, 
ceci: effectivement, la plupart de vos utilisateurs n'en ont rien à faire, mais le fait est que c'est important pour la bibliothèque. C'est un besoin institutionnel, ne vous battez pas contre ça. Dans cet exemple précis, la différence entre une bibliothèque centrée sur ses utilisateurs et une autre est que la première va mettre le lien vers le règlement intérieur à un endroit peu visible et n'appelant qu'un faible trafic (par exemple, en petits caractères en bas de la page d'accueil) et la seconde le mettre en avant dans le menu principal.

\subsection{Le mot de la fin}

Et voilà, c'est terminé. Merci d'avoir lu ce livre.

Pour finir, quelques mots qui en résument la substantifique moelle: chacune de vos décisions modifie la manière dont les gens utilisent la bibliothèque. Assurez-vous que vous améliorez les choses. Amusez-vous bien à dessiner des bibliothèques utiles, utilisables et désirables.

Amanda et Aaron, et l'équipe de l'adaptation française 


\section{ANNEXES}

\section{Reportez vos cotes d'utilisabilité (CUTI)}

Vous trouverez ici des tableaux récapitulatifs de tous les autodiagnostics proposés au fil du livre, et un endroit où reporter les CUTI de votre bibliothèque.

Une fois que vous aurez fait les recherches nécessaires pour établir vos CUTI, vous pourrez les reporter dans les tableaux puis utiliser les graphiques de type « radar " pour visualiser d'un coup d'œil vos points forts et les points à améliorer.

\begin{tabular}{|l|r|}
\hline La bibliothèque comme lieu (Chapitre 3) & /25 \\
\hline Le bâtiment de la bibliothèque est propre et fonctionne bien & $/ 20$ \\
\hline $\begin{array}{l}\text { Le bâtiment de la bibliothèque n'est pas inutilement } \\
\text { encombré }\end{array}$ & $/ 20$ \\
\hline Le mobilier répond aux besoins des usagers & $/ 20$ \\
\hline Le bâtiment s'adapte à des comportements divers & $/ 15$ \\
\hline Les usagers ont facilement accès aux prises de courant & $/ 100$ \\
\hline CUTI total & \\
\hline
\end{tabular}

\begin{tabular}{|l|r|}
\hline Espaces d'accueil (Chapitre 4) & \\
\hline Les usagers s'approchent facilement des bureaux d'accueil & $/ 25$ \\
\hline Les besoins changent, les bureaux d'accueil aussi & $/ 25$ \\
\hline $\begin{array}{l}\text { Les gens reçoivent de l'aide au moment et à l'endroit précis } \\
\text { où ils en ont besoin }\end{array}$ & $/ 25$ \\
\hline Les gens reçoivent le type d'aide dont ils ont besoin & $/ 100$ \\
\hline CUTI total & \\
\hline
\end{tabular}




\begin{tabular}{|l|c|}
\hline Règlements et services aux publics (Chapitre 5) & $/ 10$ \\
\hline Votre bibliothèque a une philosophie de service & $/ 15$ \\
\hline L'équipe connaît et fait vivre la philosophie de service & $/ 15$ \\
\hline Il y a aussi peu de procédures et de règles que possible & $/ 20$ \\
\hline $\begin{array}{l}\text { Les règlements de la bibliothèque donnent des marges } \\
\text { d'action et de décision aux professionnels }\end{array}$ & $/ 15$ \\
\hline L'équipe de la bibliothèque est accueillante et serviable & $/ 10$ \\
\hline La qualité du service est homogène au sein de l'institution & $/ 15$ \\
\hline Le service est cohérent dans toute l'organisation & $/ 100$ \\
\hline CUTI total & \\
\hline
\end{tabular}

\begin{tabular}{|l|c|}
\hline Signalétique et orientation (Chapitre 6) & /25 \\
\hline $\begin{array}{l}\text { Votre bibliothèque dispose d'une charte graphique bien } \\
\text { conçue }\end{array}$ & $/ 15$ \\
\hline $\begin{array}{l}\text { Une même identité visuelle pour l'ensemble de la } \\
\text { signalétique }\end{array}$ & $/ 10$ \\
\hline $\begin{array}{l}\text { Les différents types de signalétique sont visuellement } \\
\text { distincts }\end{array}$ & $/ 10$ \\
\hline La signalétique est minimale & $/ 10$ \\
\hline Bannir le scotch & $/ 10$ \\
\hline $\begin{array}{l}\text { La signalétique injonctive est simple, conviviale et } \\
\text { courtoise }\end{array}$ & $/ 100$ \\
\hline $\begin{array}{l}\text { La carte de bibliothèque contient des informations utiles et } \\
\text { respecte l'identité visuelle de la bibliothèque }\end{array}$ & $/ 15$ \\
\hline $\begin{array}{l}\text { Les nouveaux visiteurs peuvent repérer facilement les } \\
\text { zones de la bibliothèque }\end{array}$ & \\
\hline CUTI total & \\
\hline
\end{tabular}




\begin{tabular}{|l|c|}
\hline Présence en ligne (Chapitre 7) & $/ 10$ \\
\hline Trouver et réserver des documents est facile & $/ 20$ \\
\hline Facilitez les opérations essentielles & $/ 10$ \\
\hline $\begin{array}{l}\text { La taille de votre site est adaptée au temps que } \\
\text { vous pouvez y consacrer }\end{array}$ & $/ 10$ \\
\hline Le contenu web est attrayant & $/ 10$ \\
\hline Rédigez pour le Web & $/ 10$ \\
\hline Les sites web utilisent les conventions du design web & $/ 10$ \\
\hline $\begin{array}{l}\text { La page d'accueil exprime clairement ce que l'on peut faire } \\
\text { sur votre site }\end{array}$ & $/ 10$ \\
\hline Le site web s'adapte à tous les écrans & $/ 5$ \\
\hline Le site web utilise l'identité visuelle de la bibliothèque & $/ 5$ \\
\hline Vous utilisez les réseaux sociaux à bon escient & $/ 100$ \\
\hline CUTI total & \\
\hline
\end{tabular}

\begin{tabular}{|l|c|}
\hline La bibliothèque comme outil de travail (Chapitre 8) & $/ 25$ \\
\hline $\begin{array}{l}\text { L'équipement de votre bibliothèque est pertinent, utile et } \\
\text { utilisable }\end{array}$ & $/ 15$ \\
\hline Les collections sont adaptées aux besoins des utilisateurs & $/ 10$ \\
\hline Les supports de communication sont utiles & $/ 20$ \\
\hline Vous valorisez vos ressources & $/ 30$ \\
\hline $\begin{array}{l}\text { Les services de la bibliothèque apportent des solutions aux } \\
\text { problèmes des utilisateurs }\end{array}$ & $/ 100$ \\
\hline CUTI total & $/ 30$ \\
\hline
\end{tabular}




\section{Trouver un bon radar vierge hexagonal}

Utilisez l'un des graphiques en forme de radar pour reporter vos résultats. Notez à la main dans chaque angle de l'hexagone l'une des 6 catégories (bibliothèque comme lieu, espaces d'accueil, etc.) Reportez votre CUTI sur 100 points sur chaque ligne à l'endroit correspondant. Connectez les points.

Cela vous permettra de représenter graphiquement vos points forts en termes d'UX et ceux qui doivent être améliorés.

Vous pouvez aussi le faire sous forme informatisée en utilisant un tableur.

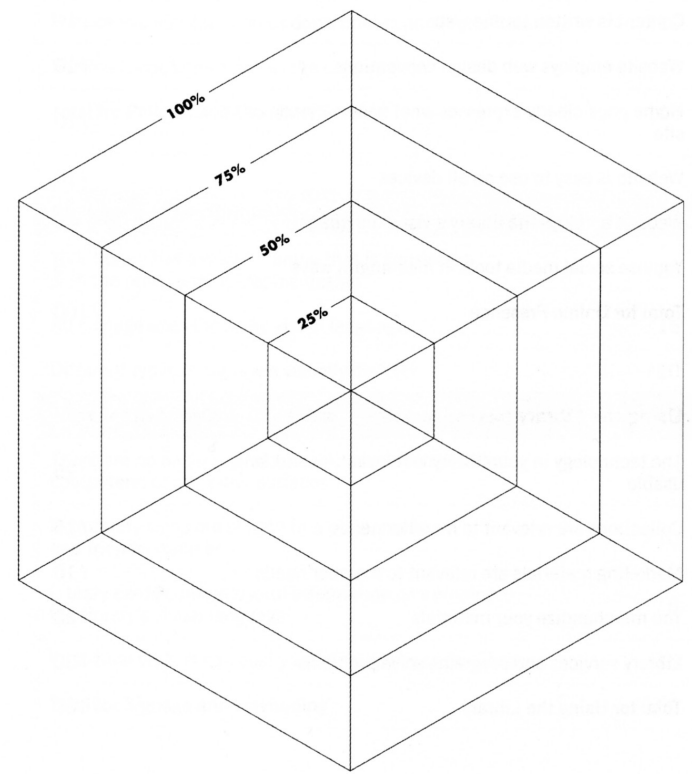




\section{Liste des illustrations}

Encadré. Suis-je un designer ?

Tableau 1. Actions

Figure 3.1. Signalétique indiquant le zonage des espaces dans les bibliothèques de l'université de Houston

Figure 3.2 Une adaptation en France, à la bibliothèque Belle Beille de l'université d'Angers, exploite la division du bâtiment en trois blocs et une signalétique colorée sur les poteaux porteurs du bâtiment

Sélection de polices de caractère

Figure 6.1. Gabarit pour une affiche d'information

Encadré. Pressé par le temps ?

Figure 6.2. Grille et gouttières

Figure 6.3. Placement des éléments sur la grille

Figure 6.4. Les éléments de la figure 6.3 retravaillés

Figure 6.5. Bibliothèque de modèles

de la Grand Valley State University

Encadré. Utilisez des couleurs efficacement

Figure 6.6. Le signe du bouton de porte

Figure 6.7. Exemple d'affiche injonctive :

Interdiction absolue de téléphoner

Figure 6.8. Exemple de message bienveillant 
Encadré. Qu'est-ce qu'un test d'utilisabilité ?

Encadré. Conseil.

Figure 7.1. Audit de contenu

Encadré. Des contenus bien pensés

Figure 7.2. Titres et contenu

Figures 7.3. et 7.4. Exemple de page de site web avant et après révision

Figure 7.5. Page d'accueil de la bibliothèque municipale de Cambridge (Canada)

Figure 7.6. Page d'accueil de la bibliothèque universitaire de l'Illinois

Figure 7.7. Page d'accueil de la bibliothèque universitaire de l'État du Montana

Figure 7.8 Page d'accueil de bibliothèque sur iPad

Figure 7.9 Page d'accueil de bibliothèque sur iPhone 


\section{Index}

\section{A}

A/B test 42

Accessibilité 15, 23, 71, 72

Accueil

Bureaux 16, 22, 36, 43, 48, 70, $91,71,72,73,74,75,76$,

77, 78, 79, 80, 83, 84, 90,

95, 96, 97, 98, 100, 101, 103, 104, 107, 108, 109,

$111,112,113,115,116,124$, $125,140,200,158,159$, 245, 173, 185, 189, 191, 192, 193, 196, 202, 240, 243, 66, 146, 189

Type de 22, 48, 70, 71, 74, 75, 76, 77, 78, 79, 80, 83, 91, $116,119,125,194,217$

Android 194

Ariane (fil d') 186

Audits

Contenus 32, 43, 44, 77, 135, $141,142,148,149,170,174$, 175, 176, 177, 215, 216, 217

Automates de prêt 83, 203, 205, 208, 210, 220, 235

Awesome Box 221

\section{B}

Barrières 42

Brown, Carol 15, 23, 71, 72

\section{C}

Catalogue, en ligne 157

Charte graphique 126, 127, 128 , $129,135,141,142,144,151$, $152,197,215$
Chartes 90, 91, 115

Client mystère 96, 97, 98, 124

Cohérence du service 122

Collections

Valorisation 9, 16, 25, 36, 45, 53, 54, 55, 159, 208, 209, 210, 211, 212, 218, 219, 225, 227

Communautés - engagement 110, 213, 228

Comportements (recherches sur) $34,35,36,37,38,40$, 42, 44, 61, 62, 72, 86, 104, 122, 143, 146, 150, 237, 238, 146

Comportements variés 38, 61 Contact (points de) 14, 24, 27, 71, 84, 90, 91, 94, 96, 100, 101, 111, 197, 230, 232

\section{Contenus}

Stratégie 22, 43, 44, 53, 137, $145,161,162,168,169$, 170, 171, 172, 174, 176, 177, 179, 184, 188, 219, 221, 226, 228

Conventions du Web 185, 189

Cooper, Simon F. 106

Couleurs (palettes de) 42, 127, 128, 132, 133, 134, 139, 140, $144,152,185,186,192,197$

Culture organisationnelle 29, 89, 229

CUTI, Cote d'utilisabilité 27, 28, $32,50,56,60,73,86,95$, $97,101,103,104,128,168$, 175, 176, 197, 209 
D

Design: conventions sites web

$11,13,15,18,19,20,21,24$, $32,34,35,45,46,58,89$, 155, 156, 194, 195, 196, 229, 240, 241, 244

Design: méthodes 13, 14, 20, 22 , $35,52,72,74,88,129,134$, $135,136,139,140,143$, 144, 152, 155, 173, 185, 189, 211, 221, 229, 233, 234, 238, 239

Design universel 22

Découverte (outils de) 159, 160, 187, 193, 219

Désirabilité 18

Difficulté (échelle de) 10, 23, 26, 70, 82, 158, 205, 210

Directions - signalétique 105

\section{E}

Eames, Charles 57

Elements of User Experience, The (Garrett) 244

Empathie 21, 111, 112, 113, 114, 124, 125, 225

Empowerment 110

Encombrement 51, 53, 57

Enquête contextuelle 37, 38, 39, $62,113,114,124$

Entrées 55, 56

Équipement

Voir aussi Technologie 77, 78, 120,64

Ergonomie 77, 208

Espaces 9, 18, 21, 22, 35, 41, 43, $45,47,48,50,51,59,60$, $63,64,70,71,73,74,79$, $83,136,148,150,162,204$, 205, 210, 211, 244, 225
Événements 56, 79, 107, 116, 193

Exploitation (systèmes de) 194, 207, 213

Expérience utilisateur

(recherche de) 13, 14, 16, 25, $28,29,30,119,239$

\section{$\mathbf{F}$}

Facebook 112, 199, 200, 201

Fils d'Ariane 186

Flexibilité 76, 77, 87

Focus groups 36

\section{G}

Gabarits 134, 135, 136, 138

Garrett, Jesse James 46, 244

Grille (modèle de) 28, 67, 136, 137

\section{H}

Handicap 19, 22

Harmonisation 119, 120

Herzog, Brian 21, 113

Hypertexte (liens) 186

\section{I}

Identification (signalétique) 141, 144

Identité visuelle $127,128,140$, 141, 142, 144, 151, 197, 198, 215

Implémentation 98, 238

Impressions 205, 206

Impulsion (emprunts d') 220

Information (signalétique) 16 , 19, 22, 23, 35, 51, 52, 59, $119,127,135,177,178,179$, 181, 182, 183, 187, 191, 200, 214, 215, 216, 226, 238 
Informatique (environnement) 133, 207

Interior Design for libraries

(Brown) 143

Itinéraires (cartes de) 38, 230, 231, 232, 235

\section{J}

Jaquettes 220

Jargon 78, 183

K

Krug, Steve 201, 241

Kupersmith, John 183

$\mathbf{L}$

Liens hypertextes 186

Localisation 76, 141, 227

Logos 127, 128, 133, 134, 135, 137, 141, 155, 185

\section{M}

Marcotte, Ethan 195

Marges 32, 102, 103, 106, 127, 136, 196, 211, 224

Marianne (Charte) 90, 96, 119

Marketing 214, 218

Marque 98, 129, 133, 151, 189, 193

Mau, Bruce 147, 148

Médias sociaux 198, 199

Membre (comme terme) 26, 31, 90, 94, 103, 106, 119

Missions 54, 57, 70, 93, 102, 111, 132, 159, 226, 228, 230, 240, 242

Mobiles 73, 74, 75, 83, 84, 171, 195
Mobilier 15, 43, 57, 58, 60, 61, $63,67,69,75,76,77,78$, 113,231

Modèles (bibliothèque de) 129, 139, 140

Modularité 78

Murs 49

N

Norman, Don 20

O

Observation 27, 60, 86, 100, 243 OPACS 159, 196

Opinions (recherches sur) 34, $35,36,37,40,44,48,60$, 72, 103, 109, 158, 184, 217

Outils de découverte 160

$\mathbf{P}$

Pantone 133

Patron-driven Acquisition

(PDA) 212, 213, 228

Personas 41, 42, 45, 89, 211, 218

Personnel, savoir-être 15, 16, $21,24,26,52,77,80,83$, 90, 93, 94, 96, 97, 98, 99, 100, 104, 106, 107, 109, 110, $111,112,113,115,119,121$, $123,125,126,133,148,154$, 194, 235, 237, 238, 240, 241, 243

Photocopies et impressions 116, 143, 203, 206, 208

Points de contact $14,15,16,24$, 25, 28, 38, 39, 57, 197, 202, 229, 230, 231, 234

Polices 128, 129, 130, 131, 132, 134, 144 
Prises électriques 64

Procédure 100, 102, 103, 107

Programmes 57

Promotion 43, 107, 213, 218

Propreté 15

Prototypes 234, 236, 237, 238

\section{$\mathbf{R}$}

Rechargement (stations de) 68, 69

Recherche

sur le site Web 11, 18, 19, 20 , $21,32,35,36,38,40,41$, $54,59,86,90,113,123$, 124, 145, 147, 157, 158, 159, 173, 187, 188, 191, 193, 196, 202, 240, 213, 214, $223,225,243,64$

Recrutement 21, 98, 105, 112

Réglementaire (signalétique) $101,102,141,143,149$

Règlements 62, 71, 99, 100, 101, 102, 104, 113, 121, 124, 202

\section{S}

Sans serifs 130

Schneider, Karen 19, 69

Scotch 146, 149

Serifs 129

Services (philosophie des) 9,

$16,18,19,21,22,25,29,30$, 35, 37, 40, 41, 47, 57, 67, 70, 71, 74, 79, 80, 81, 82, 83, $84,86,87,89,90,91,92$, 93, 94, 95, 96, 97, 98, 99 , 100, 107, 109, 113, 114, 115, 116, 117, 118, 119, 120, 124, 127, 143, 145, 153, 183, 191, 194, 200, 202, 207, 218, 221, 222, 223, 224, 225, 226, 232 , 233, 238, 240
Signalétique 14, 15, 25, 39, 41, 56, 69, 126, 127, 130, 135, $137,138,141,142,143$, $144,145,146,148,149$, 150, 154, 198, 201, 215, 221, 222, 146

SIGB 159, 160, 183, 221

Sites web

Versions mobiles 39, 161, 171, 172, 188, 193, 194, 196, 202, 244

Sondages 35, 109, 210

Sondes culturelles 40, 82, 122 , 124, 211, 223

$\mathbf{T}$

Tests $39,40,42,43,90,150$, 155, 158, 204, 159, 161, 163 , 183, 184, 191, 192, 237, 238, 241, 242, 146

Toilettes 21, 45, 113, 126, 146

Transparence 112

Twitter 112, 115, 199, 200, 201

Typographie 64, 144

u

Utilisabilité

Sites web 20, 21, 27, 28, 32, $34,39,40,123,158,159$, $161,163,168,183,185$, 189, 192, 221, 230, 237, 241, 242, 243

Utilisateur 9, 13, 14, 15, 16, 18, $19,20,23,29,30,39,41$, $42,70,75,80,81,82,85$, 100, 102, 104, 105, 108, $110,112,113,133,147,149$, $150,151,153,158,159$, 163, 169, 184, 185, 205, 206, 229, 231, 240, 241, 244 
Utilité 18, 30, 59, 172, 175, 214, 216, 218

UX (voir Expérience utilisateur)

$10,11,13,14,15,17,18,19$, $24,26,29,30,32,34,35$, 47, 58, 150, 159, 239, 240, 241, 242, 243, 244

V

Valorisation 218

Vision 9, 11, 14, 18, 44, 70, 163, 183, 228, 240, 241

Visuel (langage) 71, 169, 143, 144, 147, 149, 146, 188, 189, 195, 197

\section{w}

Web (voir Site web, Réseaux sociaux) $15,22,28,30,32$, $38,41,42,43,44,47,127$, 129, 133, 139, 140, 141, 145, $152,155,157,158,161,162$, $163,168,169,170,171,172$, $173,174,177,178,179,182$, 183, 185, 186, 187, 188, 189, 192, 193, 194, 195, 197, 198, 204, 215, 216, 221, 230, 237, 240, 243, 244

Wi-fi 16, 193, 202, 203, 204, 206, 222, 226

\section{Z}

Zonage 62, 64 


\section{Notes de lecture}

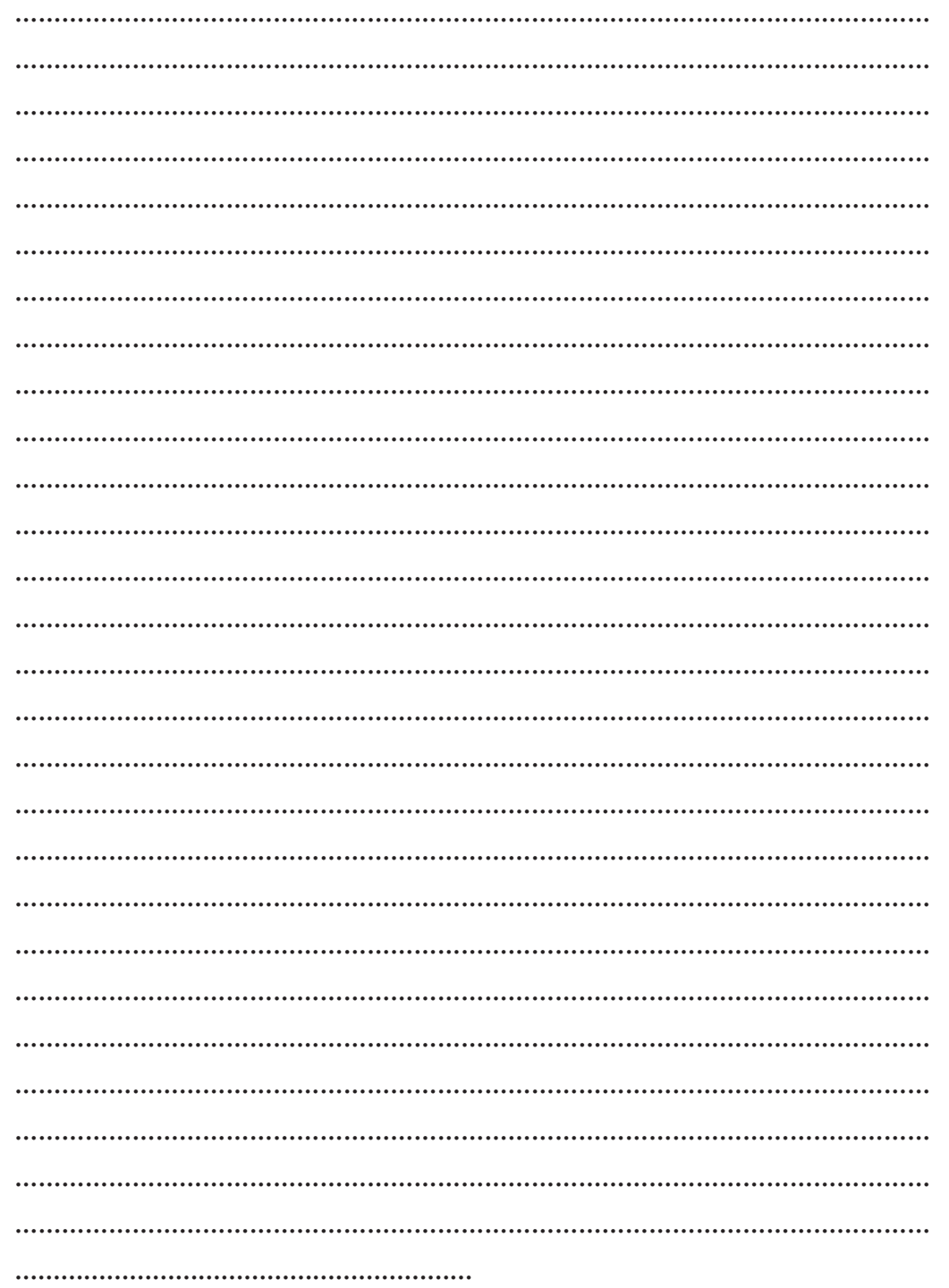


Cet ouvrage a été réalisé avec Métopes, méthodes et outils pour l'édition structurée XML-TEI développés par le pôle Document numérique de la Maison de la recherche en sciences humaines de l'université de Caen.

Secrétariat d'édition :

Silvia Ceccani

Relecture et mise en page :

Solenne Louis

Coordination maquette et mise en page :

Cédric Vigneault

Conception graphique :

atelier Perluette, 69001 Lyon

< http://www.perluette-atelier.com >

Le catalogue des presses

< http://www.enssib.fr/presses >

commander en ligne sur le comptoir des presses d'universités :

< http://www.lcdpu.fr/editeurs/enssib/ >

ENSSIB - UNIVERSITÉ DE LYON

PRESSES DE L'enssib

École nationale supérieure des sciences de l'information et des bibliothèques 17-21, boulevard du 11 novembre 1918 69623 Villeurbanne Cedex

Tél. 0472444343 - Fax 0472444344

Contact : presses@enssib.fr

Première mise en ligne

Septembre 2016.

Dépôt légal: $2^{\mathrm{e}}$ semestre 2016 